\title{
Analysis and Performance Improvement of Serial Modulation Systems with Frequency Domain Equalization including Effects of Hardware Impairments
}

\author{
BY \\ Maryam Sabbaghian \\ A THESIS SUBMITTED TO THE FACULTY OF GRADUATE STUDIES AND RESEARCH IN \\ PARTIAL FULFILMENT OF THE REQUIREMENTS FOR THE DEGREE OF \\ DOCTOR OF PHILOSOPHY \\ OTTAWA-CARLETON INSTUTUTE FOR ELECTRICAL AND COMPUTER \\ ENGINEERING \\ FACULTY OF ENGINEERING \\ DEPARTMENT OF SYSTEMS AND COMPUTER ENGINEERING \\ CARLETON UNIVERSITY \\ OTTAWA, ONTARIO
}

August 2007

Copyright (C) Maryam Sabbaghian, 2007 


$\begin{array}{ll}\begin{array}{l}\text { Library and } \\ \text { Archives Canada }\end{array} & \begin{array}{l}\text { Bibliothèque et } \\ \text { Archives Canada }\end{array} \\ \begin{array}{l}\text { Published Heritage } \\ \text { Branch }\end{array} & \begin{array}{l}\text { Direction du } \\ \text { Patrimoine de l'édition }\end{array} \\ \begin{array}{l}\text { 395 Wellington Street } \\ \text { Ottawa ON K1A ON4 }\end{array} & \begin{array}{l}\text { 395, rue Wellington } \\ \text { Ottawa ON K1A ON4 } \\ \text { Canada }\end{array}\end{array}$

Your file Votre référence ISBN: 978-0-494-33510-9 Our file Notre référence ISBN: 978-0-494-33510-9

NOTICE:

The author has granted a nonexclusive license allowing Library and Archives Canada to reproduce, publish, archive, preserve, conserve, communicate to the public by telecommunication or on the Internet, loan, distribute and sell theses worldwide, for commercial or noncommercial purposes, in microform, paper, electronic and/or any other formats.

The author retains copyright ownership and moral rights in this thesis. Neither the thesis nor substantial extracts from it may be printed or otherwise reproduced without the author's permission.
AVIS:

L'auteur a accordé une licence non exclusive permettant à la Bibliothèque et Archives Canada de reproduire, publier, archiver, sauvegarder, conserver, transmettre au public par télécommunication ou par l'Internet, prêter, distribuer et vendre des thèses partout dans le monde, à des fins commerciales ou autres, sur support microforme, papier, électronique et/ou autres formats.

L'auteur conserve la propriété du droit d'auteur et des droits moraux qui protège cette thèse. $\mathrm{Ni}$ la thèse ni des extraits substantiels de celle-ci ne doivent être imprimés ou autrement reproduits sans son autorisation.
In compliance with the Canadian

Privacy Act some supporting forms may have been removed from this thesis.

While these forms may be included in the document page count, their removal does not represent any loss of content from the thesis.
Conformément à la loi canadienne sur la protection de la vie privée, quelques formulaires secondaires ont été enlevés de cette thèse.

Bien que ces formulaires aient inclus dans la pagination, il n'y aura aucun contenu manquant.

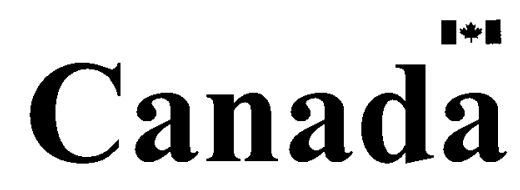




\section{Abstract}

This thesis investigates the performance characteristics of a special type of Generalized Multi-Carrier (GMC) transmission methods known as serial modulation. The well-known form of GMC is Orthogonal Frequency Division Multiplexing (OFDM) which is proved as an efficient transmission scheme in fading channels. Serial modulation with Frequency Domain Equalization (FDE) can be regarded as a Discrete Fourier Transform (DFT) precoded OFDM. In this thesis we show that this method can effectively reduce the undesirable out-of-band radiation generated by nonlinear high power amplifiers. Equivalently it can reduce the required power back-off value of the amplifier to achieve a specific out-of-band radiation. We also propose a modified version of the selected mapping algorithm to reduce the out-of-band radiation of this system.

We introduce Turbo Frequency Domain Equalization (TFDE) as an efficient scheme for serial modulation receivers which benefits from good performance of iterative systems and simplicity of equalization in the frequency domain. To optimize TFDE, we compare the performance and complexity of non-adaptive TFDE and adaptive TFDE. In the former case the equalizer is fixed during iterations while in the latter case it changes with respect to feedback information. Moreover, we compare TFDE with Low Density Parity Check (LDPC) and convoluțional codes.

The performance evaluation of TFDE like other turbo systems requires extensive simulations. To reduce the complexity of this process, we propose the Bit Error Rate Transfer (BERT) chart method to analyze the performance of this system. We develop this method for both adaptive and non-adaptive TFDE and different constellations. We 
will show that this method performs better than the well-known analysis method for turbo systems, Extrinsic Information Transfer (EXIT) chart, when the block length is small. However, for asymptotic analysis the EXIT chart is shown to be more stable.

We consider the effect of deviation in the local oscillators' frequency in the form of frequency offset and phase noise. We propose joint synchronization and turbo equalization methods to compensate for the effect of phase error when the system suffers from frequency offset alone or in combination with phase noise. In the proposed joint synchronization and equalization methods, the phase error is estimated using the soft symbols generated in the feedback loop based on the decoder output a priori information. We show that these methods can almost completely compensate for the effect of phase error. 


\section{Acknowledgement}

The list of individuals I wish to thank extends beyond the limits of this page. I would like to first express my deep and sincere gratitude to my supervisor, Dr. David Falconer, for his support and encouragement throughout my Ph.D. program. Words can not express how honored I am to work with him and benefit from his knowledge, attitude and invaluable suggestions.

I am grateful to the committee members of my Ph.D. defense: Dr. Amir K. Khandani from the Department of Electrical and Computer Engineering University of Waterloo, Dr. Yongyi Mao from School of Information Technology and Engineering University of Ottawa, Dr. Calvin Plett from the Electronics Department and Dr. Ian Marsland from Systems and Computer Engineering Department of Carleton University.

My research work was generously supported by grants, awards and scholarships provided by CITO (Communications and Information Technology Ontario), NSERC (Natural Science and Engineering Research Council Canada), Nortel Networks, OGS (Ontario Graduate Scholarship), Gabriel David Warshaw Memorial Scholarship and Carleton Entrance and Graduate scholarships.

I would like to thank the staff of Department of Systems and Computer Engineering, in particular Ms. Hebert, Ms. Lee, Ms. Kornelson, Ms. Power and Mr. Mehta for their help. I also would like to thank all my friends at our department especially at 7039-Minto Case.

Last, but not the least, I would like to thank my beloved parents Mahvash and Ata, my dear sister Minoo and my husband and best friend Hamid for their unconditional love and unbelievable support. 


\section{Table of Contents}

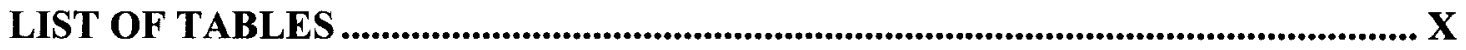

LIST OF FIGURES ........................................................................................................ XII

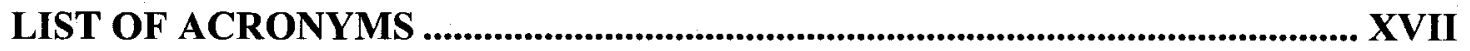

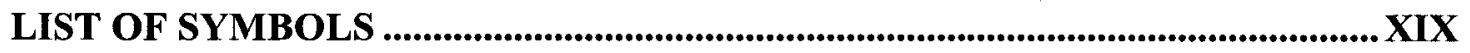

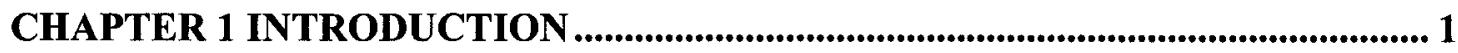

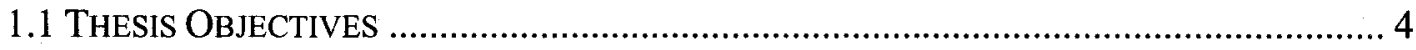

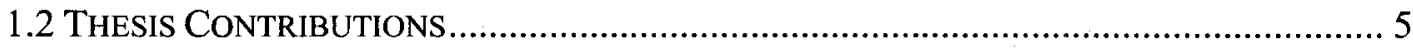

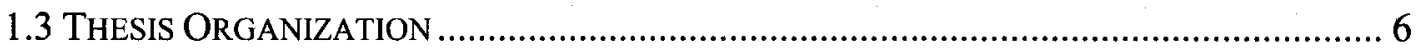

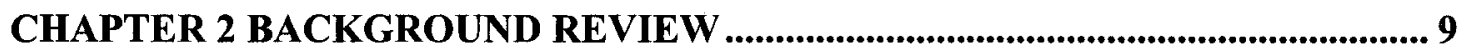

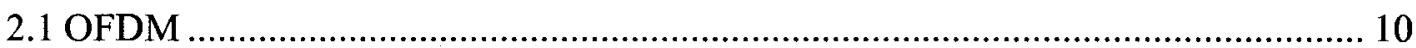

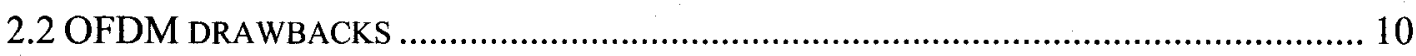

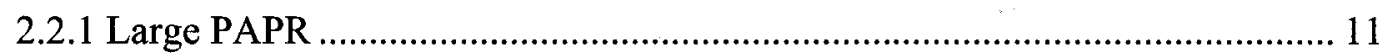

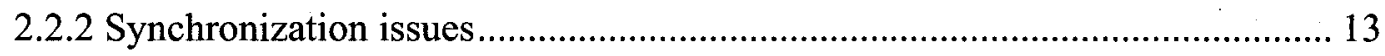

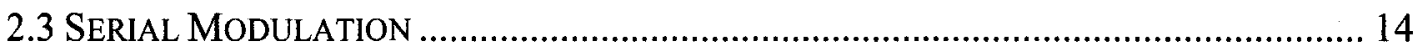

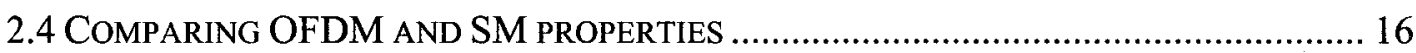

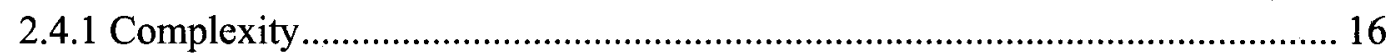

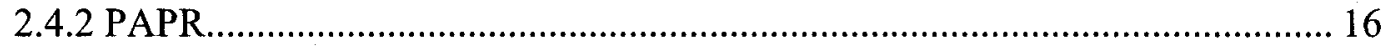

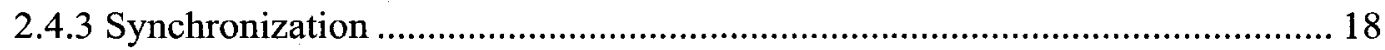

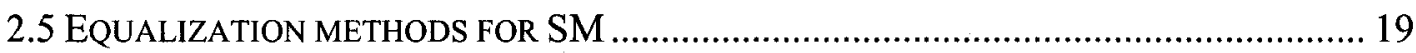

2.5.1 Linear Frequency Domain Equalization ................................................... 19

2.5.2 Decision Feedback Equalization (DFE) ............................................. 20 
2.5.3 Turbo Frequency Domain Equalization (TFDE) ...................................... 21

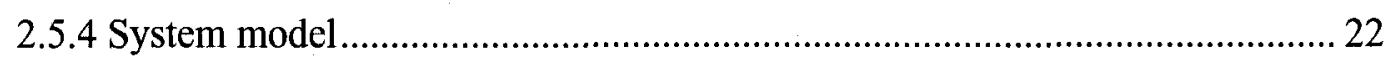

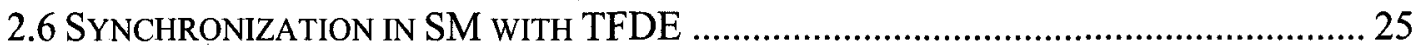

CHAPTER 3 PAPR OF OFDM AND SM SIGNALS ....................................... 26

3.1 Large Peak to Average Power Ratio Problem ..................................... 27

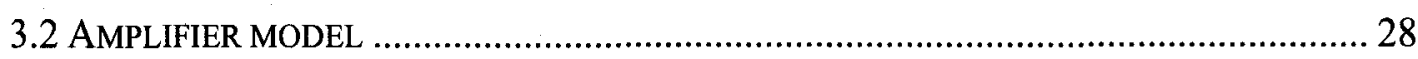

3.3 NONLINEAR AMPLIFIER EFFECT ON OUT-OF-BAND RADIATION ............................. 32

3.4 NONLINEAR AMPLIFIER EFFECT ON THE BER PERFORMANCE .............................. 39

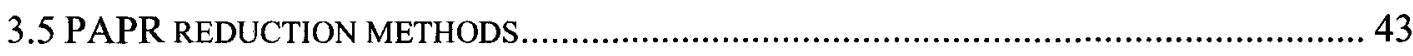

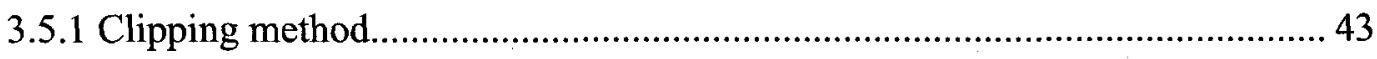

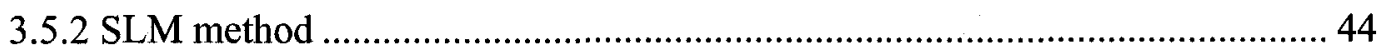

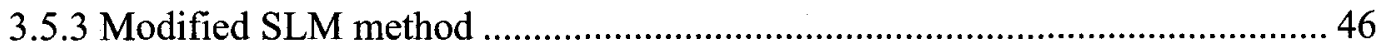

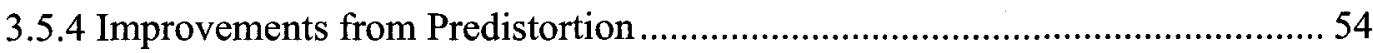

3.6 PAPR PROPERTIES OF SM-CDMA AND MC-CDMA .......................................... 56

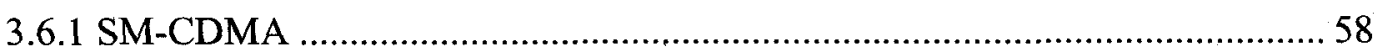

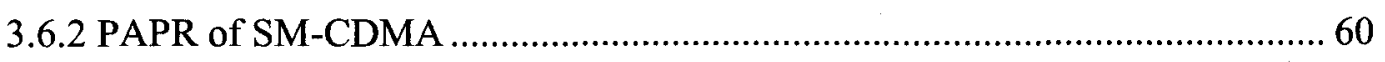

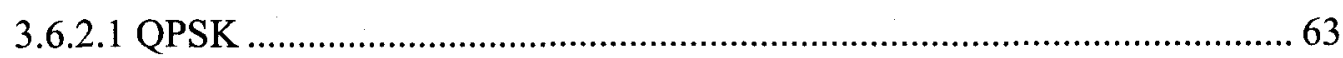

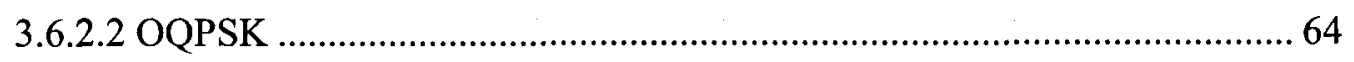

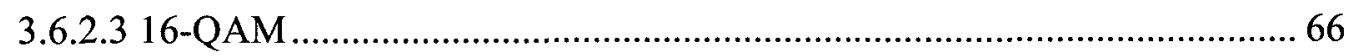

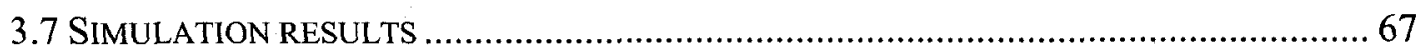

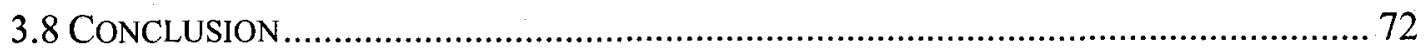

CHAPTER 4 TURBO FREQUENCY DOMAIN EQUALIZATION.......................... 74

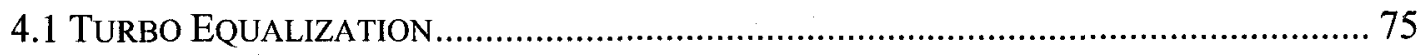

vi 
4.2 SIC-TURBo FREQUENCY DOMAIN EQUALIZATION ....................................... 75

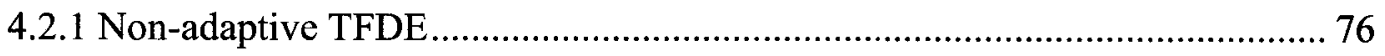

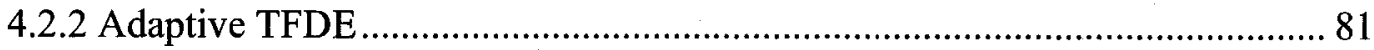

4.3 COMPARISON OF TFDE USING DIFFERENT CODING METHODS ............................. 83

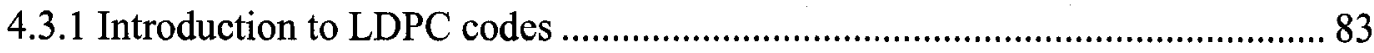

4.3.2 Performance comparison of TFDE with different coding methods................. 86

4.3.3 Complexity comparison of TFDE with different coding methods .................. 89

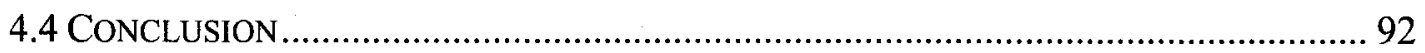

CHAPTER 5 BERT CHART ANALYSIS OF TFDE ............................................ 93

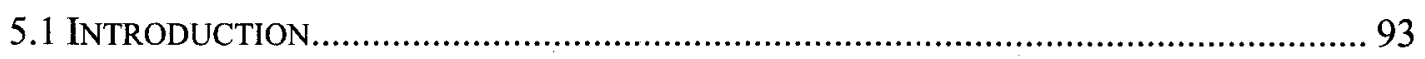

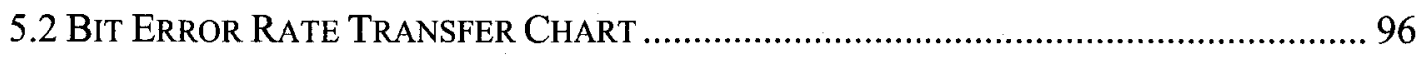

5.2.1 BERT chart for non-adaptive TFDE.................................................. 99

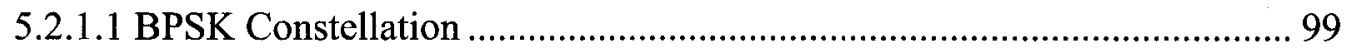

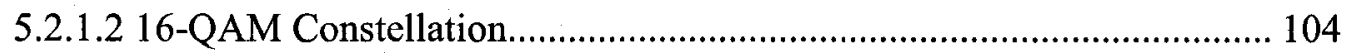

5.2.2 BERT chart for adaptive TFDE ........................................................... 104

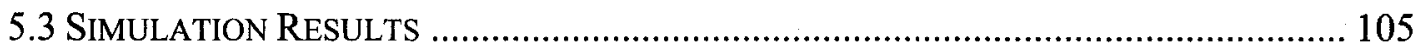

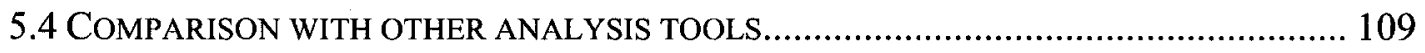

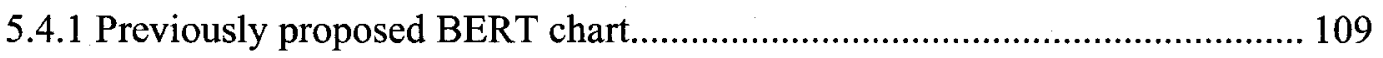

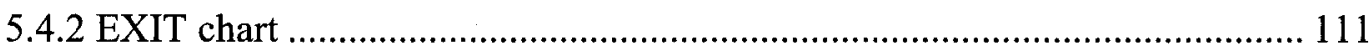

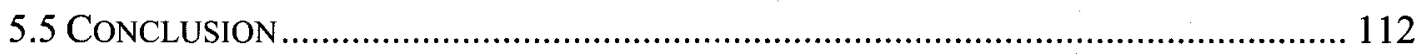

CHAPTER 6 JOINT TURBO FREQUENCY DOMAIN EQUALIZATION AND

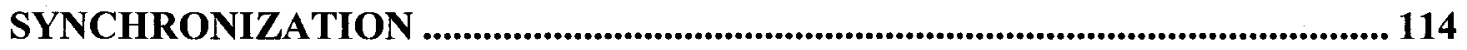

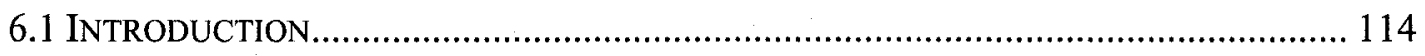

vii 
6.2 FREQUENCY OFFSET AND PHASE NOISE ...................................................... 116

6.3 JOINT SYNCHRONIZATION AND EQUALIZATION............................................... 118

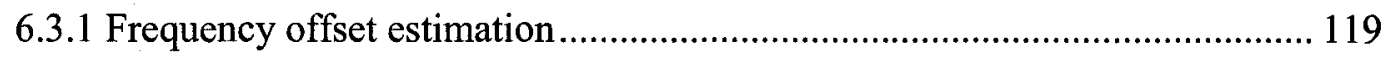

6.3.1.1 Synchronization method based on approximate ML criterion (method I)

6.3.1.2 Synchronization method based on correlating between two halves of the

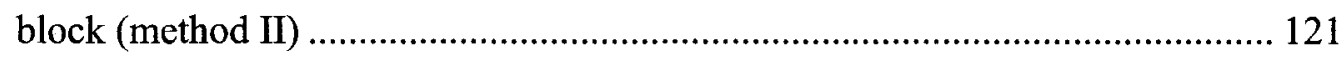

6.3.1.3 Comparison with other synchronization methods.............................. 124

6.3.2 Frequency offset and Phase noise estimation based on MAP criterion ......... 127

6.4 PERFORMANCE OF SYNCHRONIZATION METHODS ............................................. 130

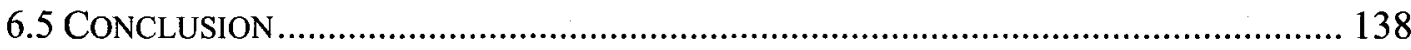

CHAPTER 7 CONCLUDING REMARKS AND FUTURE WORK...................... 139

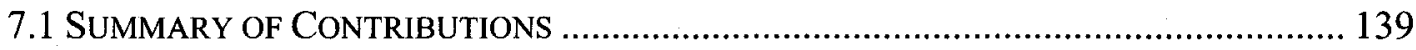

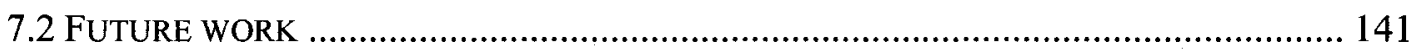

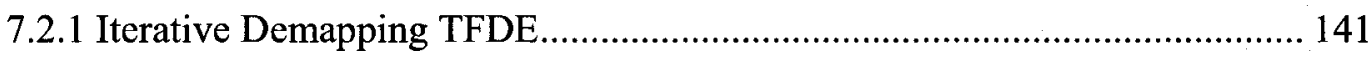

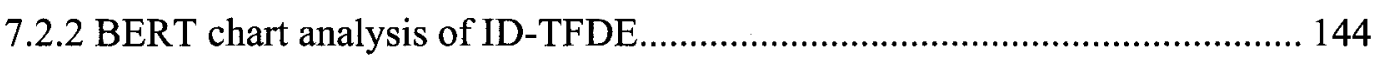

7.2.3 Sensitivity of ID-TFDE with different mappings to frequency offset.......... 148

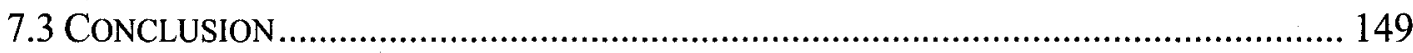

APPENDIX A $\quad$ MATRIX INVERSION LEMMA ......................................... 150

APPENDIX B NON-ADAPTIVE FREQUENCY DOMAIN EQUALIZER......... 151

APPENDIX C ADAPTIVE FREQUENCY DOMAIN EQUALIZER.................. 154

APPENDIX D V VARIANCE OF THE EQUALIZER OUTPUT ......................... 157 


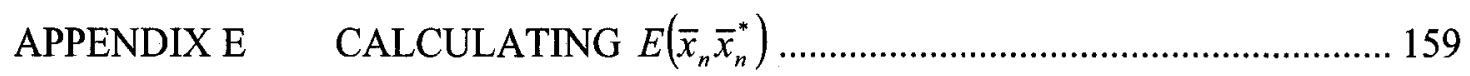

APPENDIX F BERT CHART RESULTS FOR CHANNEL C2 .......................... 160

APPENDIX G SYNCHRONIZATION PERFORMANCE FOR BPSK

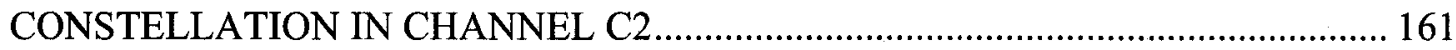

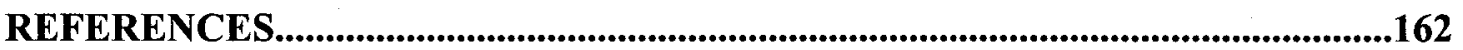




\section{List of Tables}

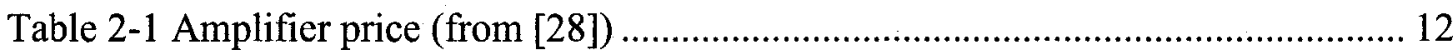

Table 3-1 Minimum required HPA back-off value for out-of-band radiation less than -40

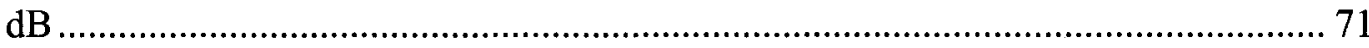

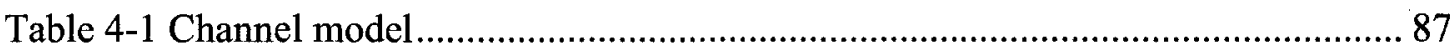

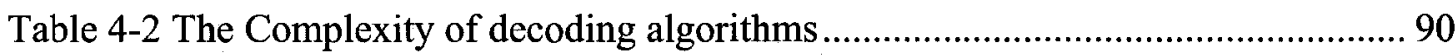

Table 4-3 Overall complexity of adaptive and non-adaptive TFDE ............................... 92

Table 6-1 Complexity comparison between the frequency offset compensation methods

Table 6-2 Complexity of Phase Noise compensation method for BPSK and QPSK ..... 131

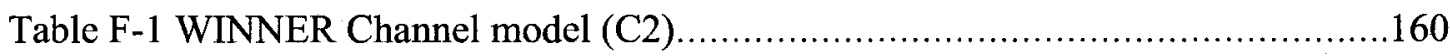




\section{List of Figures}

Figure 2-1. Cyclic prefix for each OFDM block. ................................................... 12

Figure 2-2. Block diagrams of OFDM and SM................................................. 15

Figure 2-3. Block diagram of simulated SM transmitter........................................... 17

Figure 2-4. Block diagrams of Linear and Decision Feedback Equalizers...................... 20

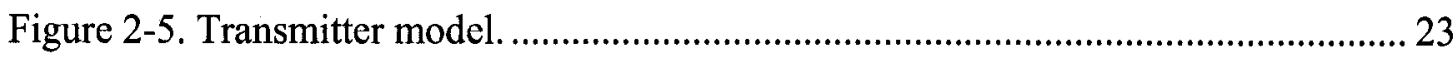

Figure 2-6. Receiver model (Turbo Frequency Domain Equalizer)............................ 23

Figure 3-1. CCDF of OFDM and SM, 16-QAM constellation, $N=256$, rectangular

frequency domain windowing, raised cosine time domain windowing $\left(\alpha_{t}=0.125\right) .28$

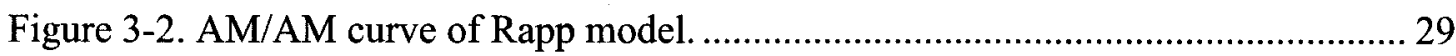

Figure 3-3. Ghorbani model for nonlinear amplifier. ........................................... 31

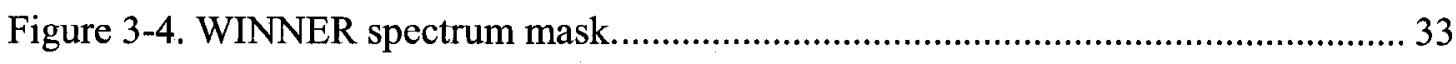

Figure 3-5. Out-of-band radiation of SM and OFDM for $p_{0}=10,16-\mathrm{QAM}$ constellation. 36

Figure 3-6. Out-of-band radiation of SM and OFDM for $p_{0}=2,16-\mathrm{QAM}$ constellation.

Figure 3-7. Out-of-band radiation of SM and OFDM for Ghorbani model, 16-QAM constellation. 37

Figure 3-8. Out-of-band radiation of SM with different frequency domain windowing,

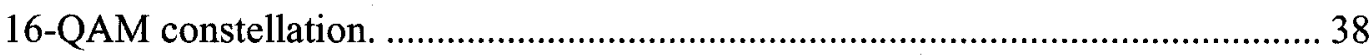

Figure 3-9. Scatter diagram of $\mathrm{SM}$ at the transmitter output, $p_{0}=2, \mathrm{BO}=5 \mathrm{~dB} \ldots \ldots \ldots \ldots . \ldots$

Figure 3-10. Scatter diagram of OFDM at the transmitter output, $p_{0}=2, \mathrm{BO}=7 \mathrm{~dB} \ldots . . .40$ 
Figure 3-11. Effect of nonlinear amplification on BER of SM in AWGN, $p_{0}=2$, 16-

QAM constellation

Figure 3-12. Effect of nonlinear amplification on BER of OFDM in AWGN, $p_{0}=2,16-$

QAM constellation.

Figure 3-13. Effect of nonlinear amplification on BER of SM in AWGN, $p_{0}=10,16-$

QAM constellation 42

Figure 3-14. Effect of nonlinear amplification on BER of OFDM in AWGN, $p_{0}=10,16-$

QAM constellation 42

Figure 3-15. Effect of Clipping and Filtering on performance of SM, $p_{0}=2$ 45

Figure 3-16. Effect of Clipping and Filtering on performance of SM, $p_{0}=10$ 45

Figure 3-17. Block diagram of conventional SLM method ([32]) 47

Figure 3-18. SLM effect on CCDF of amplitude of OFDM, $K=4$. 47

Figure 3-19. Typical OFDM and SM signal. 49

Figure 3-20. Modified SLM effect on CCDF of amplitude of Serial Modulation, $K=4 . .52$

Figure 3-21. Reducing out-of-band radiation by $\mathrm{SLM}, p_{0}=2, \mathrm{BO}=5 \mathrm{~dB}, K=4,16-\mathrm{QAM}$. 52

Figure 3-22. Reducing out-of-band radiation by SLM, $p_{0}=10, \mathrm{BO}=5 \mathrm{~dB}, K=4,16-\mathrm{QAM}$.

Figure 3-23. Reducing out-of-band radiation by $\mathrm{SLM}, p_{0}=10, \mathrm{BO}=7 \mathrm{~dB}, K=4,16-\mathrm{QAM}$.

Figure 3-24. Effect of SLM and predistortion on reducing out-of-band radiation, $p_{0}=5$, $\mathrm{BO}=5 \mathrm{~dB}, K=4,16-\mathrm{QAM}$ 
Figure 3-25. Effect of SLM and predistortion on reducing out-of-band radiation, $p_{0}=5$,

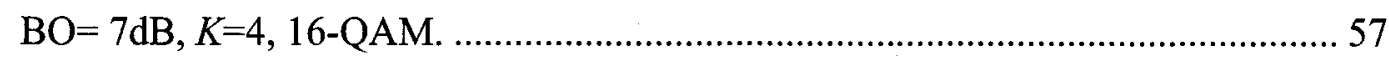

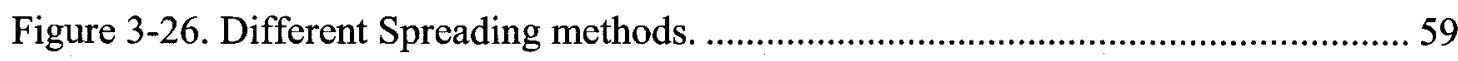

Figure 3-27. CCDF of the amplitude for SM, SM-CDMA and MC-CDMA, QPSK and

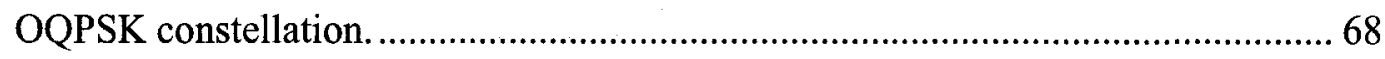

Figure 3-28. CCDF of the amplitude for SM, SM-CDMA and MC-CDMA, 16-QAM constellation. 68

Figure 3-29. Out-of-band radiation of SM, SM-CDMA and MC-CDMA, QPSK and OQPSK constellation. 69

Figure 3-30. Out-of-band radiation of SM, SM-CDMA and MC-CDMA, 16-QAM constellation. 69

Figure 3-31. Out-of-band radiation reduction of SM-CDMA using SLM method. ......... 73

Figure 4-1. Block diagram of a turbo equalizer................................................... 77

Figure 4-2. Block Diagram of SIC Turbo Frequency Domain Equalizer....................... 77

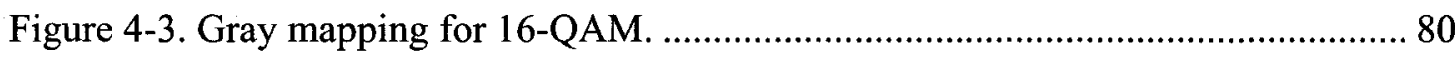

Figure 4-4. Tanner graph of the code with parity check matrix of $\widetilde{\mathbf{H}}$........................... 84

Figure 4-5. BER of TFDE with LDPC and Convolutional code, BPSK constellation..... 88

Figure 4-6. FER of TFDE with LDPC and Convolutional code, BPSK constellation..... 88

Figure 5-1. Comparison of Gaussian Approximation and simulation results for a FDE.. 98

Figure 5-2. BERT chart of non-adaptive TFDE for BPSK constellation at $\mathrm{Eb} / \mathrm{N}_{0}=4 \mathrm{~dB}$.

Figure 5-3. BERT chart of adaptive TFDE for BPSK constellation at $\mathrm{Eb} / \mathrm{N}_{0}=4 \mathrm{~dB} \ldots \ldots 106$

Figure 5-4. Comparison of BERT chart result and simulation result, BPSK................ 108 
Figure 5-5. Comparison of BERT chart result and simulation result, 16-QAM. 108

Figure 5-6. Comparison between the previous (as in [56]) and proposed BERT chart, second iteration, adaptive TFDE, 16-QAM, over channel described in Table 4-1. 110

Figure 5-7. Comparison between the previous (as in [56]) and proposed BERT chart, second iteration, non-adaptive TFDE, BPSK, over Proakis channel C

Figure 5-8. Comparison between the BERT chart and EXIT chart for a short block length, BPSK, non-adaptive TFDE.

Figure 6-1. Block Diagram of joint turbo equalization and blind synchronization. 122

Figure 6-2. Block Diagram of square-law based synchronization for OFDM (SM transmitter is presented by dashed line) 126

Figure 6-3. Performance of frequency offset compensation methods (I \& II) for BPSK132 Figure 6-4. Performance of frequency offset compensation methods (I \& II) for BPSK constellation, $\delta f T=0.2$ 132

Figure 6-5. Performance of frequency offset compensation methods (I \& II) for 4-QAM

Figure 6-6. MSE of estimation for methods I, II and VSB for BPSK and $\delta f T=0.1$ (itr= iteration number). 135

Figure 6-7. Linearity range of method I, II (HD) and VSB 135

Figure 6-8. Performance of the MAP synchronization method in the presence of frequency offset and PHN for BPSK constellation.

Figure 6-9. Performance of the MAP synchronization method in the presence of frequency offset and PHN for 4-QAM constellation

Figure 7-1. Gray mapping for 16-QAM. 143 
Figure 7-2. MSP mapping for 16-QAM.

Figure 7-3. Block diagram of ID-TFDE.

Figure 7-4. Performance of ID-TFDE with Gray, MSP and Anti-Gray mapping, 16-QAM constellation.

Figure 7-5. BERT chart result for ID-TFDE.

Figure 7-6. Effect of frequency offset on the performance of ID-TFDE, 16-QAM constellation.

Figure F-1 BERT chart result for channel C2, BPSK constellation 160

Figure G-1 The performance of synchronization method over channel C2 for BPSK....161 


\section{List of Acronyms}

\begin{tabular}{|c|c|}
\hline Acronym & Description \\
\hline AM & Amplitude \\
\hline $\mathrm{AM} / \mathrm{AM}$ & Amplitude Modulation /Amplitude Modulation \\
\hline $\mathrm{AM} / \mathrm{PM}$ & Amplitude Modulation /Phase Modulation \\
\hline $\mathrm{AP}$ & Access Point \\
\hline APP & A Posteriori Probability \\
\hline AWGN & Additive White Gaussian Noise \\
\hline BCJR & Bahl, Cocke, Jelinek, Raviv \\
\hline BER & Bit Error Rate \\
\hline $\mathrm{BO}$ & Back-Off \\
\hline $\mathrm{BP}$ & Belief Propagation \\
\hline BPSK & Binary Phase Shift Keying \\
\hline $\mathrm{CCDF}$ & Complementary Cumulative Density Function \\
\hline CDMA & Code Division Multiple Access \\
\hline $\mathrm{CP}$ & Cyclic Prefix \\
\hline CSI & Channel State Information \\
\hline C-SP & Complex spreading \\
\hline $\mathrm{D} / \mathrm{A}$ & Digital to Analog converter \\
\hline DAB & Digital Audio Broadcasting \\
\hline DFE & Decision Feedback Equalization \\
\hline DS-CDMA & Direct Sequence-CDMA \\
\hline DVB-T & Digital Video Broadcasting-Terrestrial \\
\hline Eqz. & Equalizer \\
\hline EXIT & Extrinsic Information Transfer \\
\hline FER & Frame Error Rate \\
\hline FDE & Frequency Domain Equalization \\
\hline FFT & Fast Fourier Transform \\
\hline
\end{tabular}

xvi 


\begin{tabular}{|c|c|}
\hline Acronym & Description \\
\hline FET & Field Effect Transistor \\
\hline GMC & Generalized Multi-Carrier \\
\hline HD & Hard Decision \\
\hline HPA & High Power Amplifier \\
\hline IB-DFE & Iterative Block Decision Feedback Equalization \\
\hline IDFT & Inverse Discrete Fourier Transform \\
\hline ID-TFDE & Iterative Demapping-Turbo FDE \\
\hline IFFT & Inverse Fast Fourier Transform \\
\hline ISI & Inter Symbol Interference \\
\hline LDPC & Low Density Parity Check Code \\
\hline $\mathrm{LE}$ & Linear Equalization \\
\hline LLR & Log Likelihood Ratio \\
\hline LMS & Least Mean Square \\
\hline LO & Local Oscillator \\
\hline MAP & Maximum A Posteriori \\
\hline MC-CDMA & Multi Carrier-CDMA \\
\hline MIL & Matrix Inversion Lemma \\
\hline ML & Maximum Likelihood \\
\hline MMSE & Minimum Mean Squared Error \\
\hline MPA & Message Passing Algorithm \\
\hline MSE & Mean Squared Error \\
\hline MSP & Modified Set Partitioning \\
\hline MT & Mobile Terminal \\
\hline NB & Narrow Band \\
\hline NSD & Normalized Soft Decision \\
\hline OBO & Output Back-Off \\
\hline OFDM & Orthogonal Frequency Division Multiplexing \\
\hline OQPSK & Offset Quadrature Phase Shift Keying \\
\hline PAPR & Peak to Average Power Ratio \\
\hline PHN & Phase Noise \\
\hline
\end{tabular}

xvii 


\begin{tabular}{l|l}
\hline Acronym & Description \\
\hline PLL & Phase Lock Loop \\
PN & Pseudo Noise \\
PSD & Power Spectral Density \\
pdf & probability density function \\
QAM & Quadrature Amplitude Modulation \\
QPSK & Quadrature Phase Shift Keying \\
RSC & Recursive Systematic Convolution \\
R-SP & Real spreading \\
SC & Single Carrier \\
SIC & Soft Interference Cancellation \\
SLM & Selected Mapping \\
SM & Serial Modulation \\
SM-CDMA & Serial Modulation-CDMA \\
SNR & Signal to Noise Ratio \\
S/P & Serial to Parallel \\
SSE & Sum of Squared Errors \\
SSPA & Solid State Power Amplifier \\
TCM & Trellis Coded Modulation \\
TWT & Traveling Wave Tube \\
TFDE & Wide Band \\
WB & \\
\hline & \\
\hline
\end{tabular}




\section{List of Symbols}

\begin{tabular}{|c|c|}
\hline Symbol & Description \\
\hline$a_{n}$ & $n$-th symbol \\
\hline$A_{\max }$ & largest amplitude in a constellation \\
\hline$\alpha_{t}$ & Roll-off factor of time domain raised cosine window \\
\hline$\alpha_{f}$ & Roll-off factor of frequency domain raised cosine window \\
\hline$\alpha_{s}$ & Metric of the forward recursions in BCJR \\
\hline$\beta_{s}$ & Metric of the backward recursions in BCJR \\
\hline$b_{k}$ & $k$-th bit \\
\hline$\beta$ & $3 \mathrm{~dB}$ frequency of the PSD function \\
\hline$c_{n, k}$ & $k$-th bit of the $n$-th symbol \\
\hline $\mathbf{C}_{\mathrm{h}}$ & circulant channel matrix \\
\hline$d_{n}$ & $n$-th chip \\
\hline$\delta f$ & frequency offset \\
\hline$E^{i}()$. & Expectation of a variable at the $i$-th iteration \\
\hline$f()$. & probability density function \\
\hline$f_{\lambda}^{i}(\lambda)$ & pdf of decoder output LLR in the $i$-th iteration \\
\hline $\mathbf{F}$ & FFT matrix \\
\hline$F_{A}()$. & AM/AM characteristics of power amplifier \\
\hline$F_{\Phi}()$. & $\mathrm{AM} / \mathrm{PM}$ characteristics of power amplifier \\
\hline
\end{tabular}




\begin{tabular}{|c|c|}
\hline symbol & Description \\
\hline$\phi_{n}$ & Phase error of the $n$-th symbol \\
\hline$\varphi$ & Phase error vector \\
\hline$G_{k}$ & Spreading code \\
\hline$\Gamma_{i}$ & PN sequence \\
\hline$\gamma^{2}$ & Variance of the Gaussian components in PHN model \\
\hline$\gamma_{s}$ & Metric on each branch of trellis diagram in $\mathrm{BCJR}$ \\
\hline $\mathbf{h}$ & Channel impulse response vector \\
\hline$h_{i}$ & $i$-th element of channel impulse response \\
\hline $\mathbf{H}$ & channel frequency response \\
\hline$H_{i}$ & $i$-th element of channel frequency response \\
\hline$\tilde{h}_{m, n}$ & samples of pulse shaping filter \\
\hline$I_{n}$ & $n$-th Inphase component \\
\hline $\operatorname{Im}()$. & Imaginary part of the argument \\
\hline$I_{i}$ & Number of inner iterations \\
\hline$I_{o}$ & Number of outer iterations \\
\hline$K$ & Number of generated sequences in the SLM method \\
\hline$L$ & Spreading length \\
\hline$\lambda_{n, k}$ & LLR of $k$-th bit of the $n$-th symbol \\
\hline$\lambda_{k}^{\prime}$ & LLR of the demapper output \\
\hline$M$ & Over-sampling rate \\
\hline
\end{tabular}




\begin{tabular}{|c|c|}
\hline symbol & Description \\
\hline$m_{i}$ & Conditional mean of the LLR in the $i$-th iteration in the decoder output \\
\hline$N_{h}$ & channel impulse response length \\
\hline$N_{C P}$ & Cyclic prefix length \\
\hline$N$ & Block length \\
\hline$N_{s}$ & Number of states in the trellis diagram \\
\hline$m_{s}$ & Memory of the convolutional code \\
\hline$P_{a v}$ & Average power \\
\hline$P(t)$ & Instantaneous power \\
\hline$P_{n, k}$ & Probability of $k$-th bit of the $n$-th symbol being zero \\
\hline$P_{E q}$ & BER at the equalizer output \\
\hline$P_{i}$ & BER at the decoder output in the $i$-th iteration \\
\hline$P_{m}$ & Probability of having a symbol with amplitude of $A_{\max }$ \\
\hline$p_{0}$ & Smoothness factor of Rapp model \\
\hline$P_{s}$ & Input power corresponding to the saturation level \\
\hline $\bar{P}_{i}$ & Average input power \\
\hline$\breve{\mathbf{P}}$ & Frequency response of frequency domain window \\
\hline$Q_{n}$ & $n$-th Quadrature component \\
\hline $\mathbf{r}$ & received vector (time domain) \\
\hline$r_{i}$ & $i$-th element of received vector (time domain) \\
\hline $\mathbf{R}$ & received vector (frequency domain) \\
\hline
\end{tabular}




\begin{tabular}{|c|c|}
\hline symbol & Description \\
\hline$\overline{R_{i}}$ & $i$-th element of received vector (frequency domain) \\
\hline$R_{s}$ & Code rate \\
\hline $\operatorname{Re}()$. & Real part of the argument \\
\hline$S$ & Constellation \\
\hline$S_{k, b}$ & Subset from the constellation in which the $k$-th bit is $b$ \\
\hline$\Sigma_{i}^{2}$ & Variance of the equalizer output in the $i$-th iteration \\
\hline$s_{m}$ & $m$-th component of the over-sampled signal \\
\hline$\sigma_{d}^{2}$ & Average power of the constellation \\
\hline$T$ & Block duration \\
\hline$T_{c}$ & Chip duration \\
\hline$v$ & Average variance of the equalizer output components \\
\hline$v_{i}^{2}$ & Variance of the LLR in the $i$-th iteration in the decoder output \\
\hline $\mathbf{W}$ & Frequency domain equalizer coefficient vector \\
\hline$W_{i}$ & $i$-th element of frequency domain equalizer coefficient vector \\
\hline$\overline{\mathbf{x}}$ & Soft symbol vector (time domain) \\
\hline $\bar{x}_{i}$ & $i$-th element of soft symbol vector (time domain) \\
\hline$\overline{\mathbf{X}}$ & Soft symbol vector (frequency domain) \\
\hline $\bar{X}_{i}$ & $i$-th element of soft symbol vector (frequency domain) \\
\hline$x_{i}$ & $i$-th transmitted symbol \\
\hline$\hat{x}_{i}$ & Ghorbani model parameter \\
\hline
\end{tabular}




\begin{tabular}{l|l}
\hline symbol & Description \\
\hline $\bar{y}_{i}$ & Ghorbani model parameter \\
\multirow{2}{*}{$z_{i}$} & $\begin{array}{l}\text { Equalizer output vector (time domain) } \\
i \text {-th element of equalizer output vector (time domain) }\end{array}$
\end{tabular}




\begin{tabular}{|c|c|}
\hline Function & Definition \\
\hline $\mathbf{0}_{M \times N}$ & $M \times N$ matrix with elements of zero \\
\hline $\mathbf{1}_{M \times N}$ & $M \times N$ matrix with elements of one \\
\hline - & Element-by-element multiplication of two vectors \\
\hline$x$ & Scalar Multiplication \\
\hline $\mathbf{r}_{1} \circ \mathbf{r}_{2}$ & Scalar Products of the vectors $\mathbf{r}_{1}$ and $\mathbf{r}_{2}$ \\
\hline$\lceil r\rceil$ & Largest integer smaller than or equal to $r$ \\
\hline$r !$ & $r(r-1)(r-2) \ldots \times 2 \times 1$ \\
\hline$(r)_{k}$ & $\frac{r(r-1) \ldots(r-k+1)}{k !}$ \\
\hline$\delta_{r}$ & $\left\{\begin{array}{l}1 \text { if } r=0 \\
0 \text { if } r \neq 0\end{array}\right.$ \\
\hline$u(r)$ & $\left\{\begin{array}{l}1 \text { if } \quad r \geq 0 \\
0 \text { if } r<0\end{array}\right.$ \\
\hline $\operatorname{sgn}(r)$ & $\left\{\begin{array}{lll}+1 & \text { if } & r \geq 0 \\
-1 & \text { if } & r<0\end{array}\right.$ \\
\hline$Q(x)$ & $Q(x)=\frac{\Delta}{\sqrt{2 \pi}} \int_{x}^{+\infty} e^{-\frac{t^{2}}{2}} d t$ \\
\hline
\end{tabular}




\section{Chapter 1 Introduction}

Communication systems are among the most prominent human inventions which have become extremely popular particularly in the appealing form of wireless communications. Communication systems, first dedicated only to voice transmission, are now used for image, video and data transmission too. The growing demand for high rate untethered transmission (offering bit rates of $100 \mathrm{Mb} / \mathrm{s}$ or more) has stimulated efforts to look for wireless transmission methods providing high spectral efficiency to achieve the best use of the allocated bandwidth. Inter Symbol Interference (ISI), as a fundamental physical layer issue, appears strongly challenging either in wired and terrestrial networks or in wireless networks when the transmission rate is increased. To overcome the ISI, generated by long delay spread multipath fading channels, different equalization methods have been proposed. Among them are Linear Equalization (LE), Decision Feedback 
Equalization (DFE) and Maximum Likelihood (ML) equalization implemented in the time domain [1]. All these methods suffer from high computational complexity, as their complexity increases at least linearly with the channel impulse response length. Recently, Orthogonal Frequency Division Multiplexing (OFDM) as an alternative method, offering good performance with reasonable complexity has become extremely popular for transmission over multi-path fading channels [2]. Although OFDM was introduced and even used in late sixties [3], it did not attract much attention because of implementation issues. Nevertheless, improvement in signal processing tools, especially the fast Fourier transform (FFT), mitigated the complexity issues [4]. After a steadily active research and technology evolution during some decades OFDM is now used in various standards as the transmission method. ${ }^{1}$ OFDM, which is a block-wise transmission method, is based on transmitting high rate data over different sub-carriers with a low rate on each one. Parallel transmission over sub-carriers is performed by using inverse FFT (IFFT) which is very efficient in terms of complexity. The orthogonality of sub-carriers cancels the inter carrier interference while inter block interference is prevented by using a cyclic prefix at the beginning of each block. Since the channel is almost flat for each sub-carrier frequency range, the equalization process is not complex if the channel frequency response is known. The equalization only requires either a multiplication for each subcarrier or it can be equivalently performed by considering channel frequency response coefficients in metrics of Bit Interleaved Coded Modulation (BICM) [5][6][7].

Comparing OFDM with the traditional single carrier method with time domain equalization, OFDM is preferred for providing high bit rate transmission and achieving good performance with less complexity. Large peak to average power ratio (PAPR) is

${ }^{1}$ OFDM is used in standards like: DAB, DVB-T, HIPERLAN/2, IEEE 802. 
known as the most important disadvantage of OFDM [8]. This problem appears when a large number of symbols add together constructively during the IFFT operation. Since a single carrier system does not include the IFFT in the transmitter, the generated signals in this system have smaller PAPR than those in OFDM. Systems with large PAPR require power amplifiers with a wide range of linearity which are expensive and power inefficient. This problem can be solved by using different PAPR reduction methods, using amplifiers with large back-off values to avoid in-band and out-of-band distortion, or linearization of the power amplifier with pre-distortion methods [8][9][10][11]. All these solutions increase either the cost or the complexity of the system. In [12] single carrier or Serial Modulation (SM) systems with Frequency Domain Equalization (FDE), which is considerably less complex than time domain equalization, was proposed as an alternative method for OFDM. Single carrier systems, by offering almost the same performance and overall complexity as OFDM but smaller peak to average power ratio, have attracted much attention during the last decade [13][14]. Single carrier systems have been shown to be quite efficient from this point of view especially for up-link transmission, from terminal to the base station [13]. Another advantage of single carrier compared to OFDM is its lower sensitivity to carrier frequency synchronization. In OFDM, frequency offset can destroy the orthogonality of sub-carriers and generate inter carrier interference. 


\subsection{Thesis Objectives}

In this thesis, we focus on different physical layer challenges for SM or block-wise single carrier systems and propose some possible solutions. The different issues considered here are:

1. Investigating power-efficient transmission methods in the presence of nonlinear high power amplifier (HPA) for signals with large peak to average power ratio: A brief review of the PAPR problem in OFDM and SM is mentioned in sections 2.2.1 and 2.4.2 respectively. The more detailed discussion on the new techniques proposed in this thesis for out-of-band radiation reduction for SM and SM-Code Division Multiple Access (SM-CDMA) can be found in Chapter 3.

2. Investigating efficient iterative equalization methods with frequency domain processing for single carrier systems in multipath fading channels: A short review of current TFDE systems and the optimized TFDE in this thesis is briefly mentioned in section 2.5.3, and investigation and evaluation of TFDE schemes is carried out in Chapter 4.

3. Investigating methods to reduce the high complexity of performance evaluation of turbo equalization systems: The basic idea of the method proposed in this thesis for performance analysis of TFDE and its main difference with the Extrinsic Information Transfer (EXIT) chart are briefly reviewed in section 2.5.3 and new results on the Bit Error Rate Transfer (BERT) chart approach for TFDE performance evaluation are presented in Chapter 5. 


\section{Investigating efficient synchronization methods in the presence of frequency}

offset and phase noise for single carrier systems with turbo frequency domain equalization: The concept of carrier synchronization and the proposed methods in this dissertation to solve this problem are mentioned in section 2.6. The details of these methods are presented in Chapter 6.

\subsection{Thesis Contributions}

The main contributions of this thesis are as follows:

- Comparison between out-of-band radiations generated by nonlinear amplifier in OFDM and SM systems and proposal and evaluation of a modified selected mapping (SLM) algorithm for PAPR reduction in SM systems.

- Investigating the effect of real and complex spreading on the amplitude distribution of SM-CDMA when multi-level or equal-level constellation is used.

- Performance/complexity comparison between SM systems with adaptive and nonadaptive TFDE when convolutional or LDPC code is used.

- Proposing a novel BERT chart as a tool for the analysis of turbo frequency domain equalization systems (for both adaptive and non-adaptive TFDE and different constellations).

- Proposing an iterative joint synchronization and equalization method for SM systems; two methods for frequency offset compensation and one method to remove the effect of both frequency offset and phase noise. 


\subsection{Thesis Organization}

The thesis is organized as follows:

Chapter 2 is an extended abstract of the thesis in which we have a brief background review of hardware impairments effect on SM and OFDM systems and current solutions. We also briefly mention the suggested solutions in this thesis.

In Chapter 3, while comparing the amplitude distribution of OFDM and SM signals, the effect of nonlinear power amplifiers on out-of-band radiation and performance degradation is investigated. In this chapter a modified version of the SLM method for reducing out-of-band radiation in SM systems is proposed. We also investigate the effect of complex and real spreading methods and different constellations on the PAPR of SMCDMA. Based on this work the following papers ([16][17][18][19][20]) have been published or submitted for possible publication:

- M. Sabbaghian and D. Falconer, "Reducing Required Power Back-Off of Nonlinear Amplifiers in Serial Modulation using SLM Method," in Proc. Vehicular Tech. Conf., Dallas, TX, Sept. 2005.

- M. Sabbaghian and D. Falconer, "Peak to Average Power Ratio Properties of MCCDMA and SM-CDMA," in Proc. Vehicular Tech. Conf., Melbourne, Australia, May 2006.

- F. Danilo-Lemoine, D. Falconer, C-T Lam, M. Sabbaghian and K. Wesołowski, "Power Backoff Reduction Techniques for Generalized Multicarrier Waveforms," submitted to EURASIP journal on Wireless Communications and Networking, Apr. 2007, revised July 2007.

- D. Falconer, F. Danilo-Lemoine, C. Lam and M. Sabbaghian, "Power Backoff Reduction for Generalized Multicarrier Waveforms," in Proc. EUSIPCO 2007.

A complete investigation of PAPR reduction methods is outside the scope of this thesis. However, the results presented in Chapter 3 motivate the use of SM for uplink transmission, and therefore provide a basis for the investigations reported in later chapters. 
In Chapter 4, we review non-adaptive and adaptive TFDE. In the first case, in contrast to the latter case, the equalizer coefficients do not change as iterations continue. We also consider two coding schemes; convolutional codes and LDPC codes. We compare these four cases from performance and complexity viewpoints. The results have been published in $([21])$ :

- M. Sabbaghian and D. Falconer, "Comparison between Convolutional and LDPC Code based Turbo Frequency Domain Equalization," in Proc. International Conf. Commun., Istanbul, Turkey, June 2006.

In Chapter 5 we propose and modify the BERT chart as a tool for performance analysis of turbo frequency domain equalizer. The results achieved by the BERT chart are verified by the simulation results. We also compare this method with EXIT chart. The results of this work have been published or submitted for possible publication in $([22][23][24]):$

- M. Sabbaghian, D. Falconer and H. Saeedi, "BER Transfer Chart Analysis of Turbo Frequency Domain Equalization," in Proc. Vehicular Tech. Conf., Montreal, Canada, Sept. 2006.

- M. Sabbaghian and D. Falconer, "Comparison of adaptive and non-adaptive turbo frequency domain equalization via BER transfer chart," in Proc. Vehicular Tech. Conf., Baltimore, MD, USA, Sept. 2007.

- M. Sabbbaghian and D. Falconer, "BER Transfer Chart Analysis of Turbo Frequency Domain Equalization," submitted to IEEE Trans. Wireless Commun., Jan. 2007.

In Chapter 6, we investigate the effect of frequency offset and phase noise on the performance of TFDE. We also propose a joint synchronization and turbo equalization method. Two methods are suggested for frequency offset compensation. We also propose a method based on Maximum A Posteriori (MAP) criterion when the system suffers from both frequency offset and phase noise. The results of this work have been published in ([25][26]): 
- M. Sabbaghian and D. Falconer, "Joint Turbo Frequency Domain Equalization and Blind Carrier Synchronization," in Proc. CWIT, Jun. 2007.

- M. Sabbaghian and D. Falconer, "Joint Turbo frequency Domain Equalization and Carrier Synchronization," accepted in IEEE Trans. Wireless Commun., Jun. 2007.

Future works are described in Chapter 7. In this chapter we consider the possibility of extending the work in this thesis to Iterative Demapping-TFDE (ID-TFDE) systems. Some primary results for the performance of ID-TFDE with different mappings are presented. We also discuss how to modify the BERT chart so that it can be used for IDTFDE. We also consider the possibility of investigating the sensitivity of ID-TFDE with different mappings to frequency offset. 


\section{Chapter 2 Background review}

\section{RF hardware impairments in OFDM and SM systems, current and proposed solutions in this thesis}

In this chapter, we briefly review the robustness of orthogonal frequency division multiplexing and serial modulation techniques against some RF hardware impairments of nonlinearity, frequency offset and phase noise. We also discuss the complexity of simulation-based analysis of the iterative systems. For each case we address and briefly discuss the current solutions. We also review our general approach in this thesis to solve the problems and what distinguishes our approach from the existing ones. 


\subsection{OFDM}

Orthogonal frequency division multiplexing is known as one of the most efficient methods for transmission over multipath fading channels for its reasonable complexity and the robustness against frequency selectivity. The OFDM technique is based on the idea of transmitting a high rate data stream on $N$ sub-carriers simultaneously while each one carries a smaller rate [27]. The sub-carrier spacing is the reciprocal of the block duration. These sub-carriers have to be orthogonal to prevent inter carrier interference. The equivalent baseband OFDM signal can be generated by taking the inverse discrete Fourier transform (IDFT) of a data block with length $N$. To prevent inter block interference, a guard time should be inserted at the beginning of each block as presented in Figure 2-1. Thus a copy of a few last symbols of the block is transmitted in the guard time which is known as the cyclic prefix. The cyclic prefix length should be at least equal to the channel delay spread length. The orthogonality of sub-carriers would be destroyed if there is no cyclic prefix.

The transmitted data symbols can be recovered by taking a discrete Fourier transform (DFT) of the received data block. By selecting an appropriate block length, we can replace DFT by the computationally efficient FFT method.

\subsection{OFDM drawbacks}

In this section we discuss nonlinear amplification and synchronization which are known to be major issues in OFDM systems. These two concepts are important problems in any communication system, but the structure of multi-carrier systems makes them more 
crucial for OFDM. We briefly review the cause of these problems and the methods proposed to solve them.

\subsubsection{Large PAPR}

An OFDM signal is a linear combination of complex symbols modulated on different sub-carriers to be transmitted simultaneously. The signal can exhibit high fluctuations when the components add together constructively. The envelope fluctuations increase the ratio of the instantaneous peak power to the average power of the OFDM signal. Then, to avoid the out-of-band or in-band distortion caused by the nonlinear characteristics of high power amplifier, we need amplifiers with a large dynamic range or equivalently large back-off value (the ratio of input power at saturation level and the average input power) [8]. Usually, as exemplified in Table 2-1, the cost of the amplifier increases quickly with its power back-off value. The parameter $\mathrm{P} 1 \mathrm{~dB}(1 \mathrm{~dB}$ compression point $)$ in Table 2-1 shows the input power for which the output power deviates $1 \mathrm{~dB}$ from the ideal case [28].

The distortion is not the only issue in the amplification process. If the PAPR is large, the amplifier efficiency decreases and thermal management appears as a major issue. We may also face some physical restrictions like requiring a fan or heat sink to adequately cool down the device. Therefore having signals with large PAPR, not only increases the cost but also the size of the device. This concept is most important for the uplink since it applies to cost- and power-sensitive user terminals. 


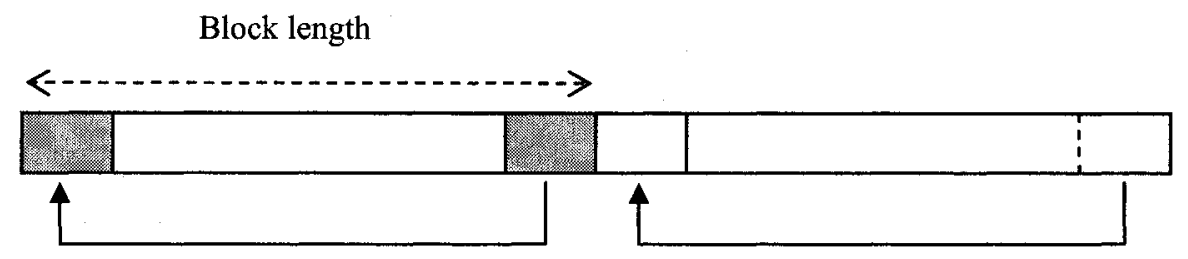

Figure 2-1. Cyclic prefix for each OFDM block.

Table 2-1 Amplifier price (from [28])

\begin{tabular}{|l|l|c|l|}
\hline Manufacture & Part Number & Approx. Price per 5k & P1 dB \\
\hline RF Micro Devices & RFM-2163 & $\$ 2.77$ & $30.0 \mathrm{dBm}$ \\
\hline AMCOM & AM072MX-QG & $\$ 9.00$ & $31.5 \mathrm{dBm}$ \\
\hline Stellex & PA1177R & $\$ 87.00$ & $33.0 \mathrm{dBm}$ \\
\hline NEC & NE6500496 & $\$ 75.00$ & $35.5 \mathrm{dBm}$ \\
\hline
\end{tabular}


The nonlinearity of HPA characteristics can generate distortion in the signal which appears as spectral splatter interfering with adjacent channels [29][30]. Larger amounts of distortion may cause in-band distortion and degrade the system bit error rate performance.

To prevent these effects, various PAPR reduction methods have been proposed. The simplest one is clipping and filtering, in which the signal is deliberately clipped. To remove the out-of-band components generated by this process, the clipped signal is filtered [8][31]. Selected mapping (SLM) and partial transmit signaling (PTS) are two examples of other approaches to solve the PAPR problem [32][33]. In both methods by using reversible functions, different versions of the original data block are generated. Among these blocks, the one with the lowest PAPR is transmitted. In the receiver the inverse function is used to recover the original data. Coding is also another PAPR reduction technique in which among some generated code words only those with the appropriate PAPR are transmitted [10][34]. In contrast to clipping, the other mentioned methods do not distort the original data signal but they need more bandwidth for transmitting some side information or adding some redundancy to the system.

\subsubsection{Synchronization issues}

To be able to recover the symbols transmitted simultaneously on different sub-carriers, these sub-carriers have to be orthogonal. Any deviation from the carrier frequency can destroy this orthogonality and generate inter carrier interference [35][36]. The deviation could be a constant frequency offset or a time-varying phase shift generated by the spectral splatter of the oscillators which is known as phase noise [27]. Generally, even if 
frequency offset is only a fraction of sub-carrier spacing, it can degrade the OFDM performance significantly. Therefore, increasing the block length, makes the problem become more challenging. The effect of phase noise is more destructive than frequency offset because it adds a time varying phase error [37]. Several synchronization methods have been suggested for both time and frequency recovery in OFDM. In [38] a maximum likelihood estimator is designed to estimate the frequency offset when a training sequence is transmitted. In [39] using the correlation between the cyclic prefix and its copy is suggested for timing recovery and in [40] this correlation is used for both timing and frequency recovery. The effect of phase noise has also been analyzed in different papers $[35][41][42]$.

\subsection{Serial Modulation}

In the previous section, we reviewed some disadvantages of OFDM systems which can degrade the performance unless some compensation methods are used. Even if these methods compensate for the performance degradation completely, they increase the overall complexity. This encourages us to look for methods as efficient as OFDM but more robust against amplification and synchronization issues.

OFDM can be regarded as a special group of block-wise transmission methods known as GMC. By this approach, IFFT is one of the possible choices for frequency domain processors. Employing alternative processors gives us the opportunity to design other systems with the desired properties. Single carrier or serial modulation with frequency domain equalization is another block-wise transmission method which is shown to exhibit more robustness against hardware impairments of amplifier nonlinearity, 
frequency offset and phase noise $[12][13][14][15]$. Similar to OFDM, SM uses a cyclic prefix to overcome inter block interference and generate a periodicity in the signal which is required for employing DFT and IDFT.

Figure 2-2 depicts the block diagram of both OFDM and SM systems. As presented in this figure, SM can be regarded as DFT-precoded OFDM. In other words, in the transmitter no frequency domain processing is performed. In the receiver, by using a DFT the signal is transferred to the frequency domain, then it is equalized in the frequency domain. Finally by an IDFT it is returned to the time domain.

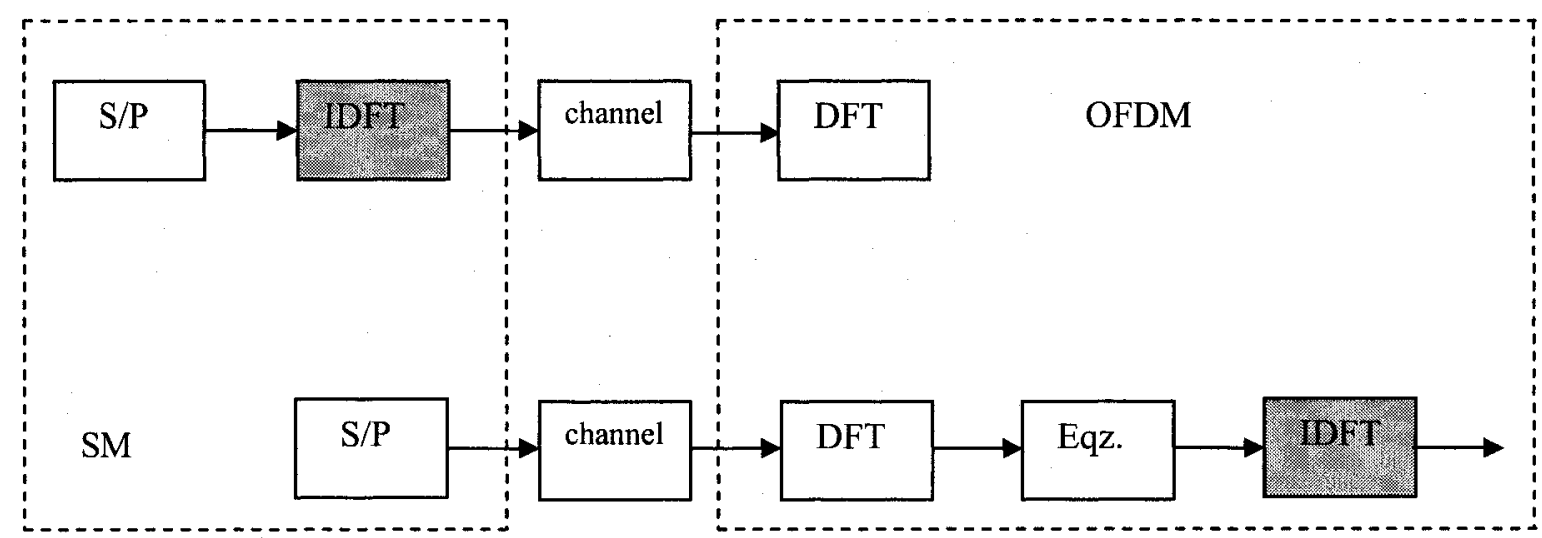

Transmitter

Receiver

Figure 2-2. Block diagrams of OFDM and SM. 


\subsection{Comparing OFDM and SM properties}

\subsubsection{Complexity}

As can be seen in Figure 2-2, SM and OFDM are analogous in terms of overall complexity $^{2}$ while as presented in [13] they can achieve almost the same performance. The complexity of OFDM and SM with FDE is considerably less than that of time domain equalization, which increases at least linearly with the channel impulse response length. In SM and OFDM the complexity of FFT and IFFT algorithms increases linearly with the logarithm of the block length which is generally $k$ times the channel impulse response (usually $6<k<10$ ). The equalization process also requires only a multiplication for each sub-carrier frequency.

\subsubsection{PAPR}

As discussed in section 2.2.1 and as will be addressed in Chapter 3, OFDM signals with large PAPR require amplifiers with large back-off, otherwise the system suffers from outof-band radiation and performance degradation [25][30]. On the other hand, to enhance the power efficiency and decrease the cost, HPAs with smaller back-off are desirable. As the SM transmitter does not include the IDFT module, the PAPR of a SM signal is smaller than that of OFDM, therefore allowing more efficient and economical HPAs [13]. The need for HPA with smaller back-off for the mobile terminal is more critical than for the base stations. Hence we only focus on the up-link transmission.

\footnotetext{
${ }^{2}$ The SM transmitter complexity is less and its receiver complexity is more than that of OFDM.
} 
In the downlink, there is only one power amplifier at the base station, whose cost is shared among many user terminals. Besides, adding different users' signals together, which occurs in the base station, increases the PAPR in the downlink [14] even if SM is used.

In Chapter 3 we consider an SM transmitter whose block diagram is presented in Figure 2-3, and investigate the effect of nonlinear amplifiers on the performance and out-of-band radiation. We will show that although SM has smaller PAPR than OFDM, when symbols are selected from a 16-QAM constellation, even a small amount of nonlinear amplification can generate considerable spectral regrowth. As will be presented in Chapter 3, the BER performance degradation caused by the nonlinear amplification of SM signals, in contrast to that of OFDM, is usually negligible. Except for [13] and [28], very few, if any, papers are available which depict the actual out-of-band radiation of SM and OFDM.

We also look for methods to improve the signal amplitude distribution and reduce outof-band radiation. In Chapter 3, we propose a modified version of the SLM method which is designed for SM. The method of generating different versions of the original block and the selection rule is different from the original SLM.

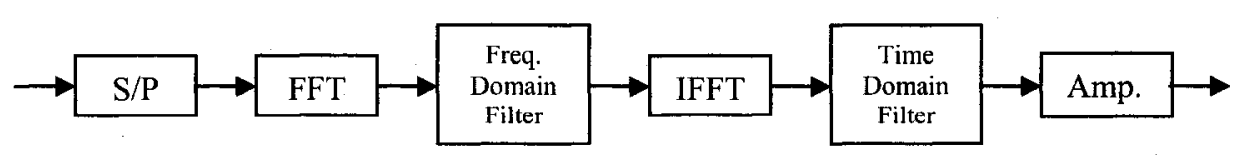

Figure 2-3. Block diagram of simulated SM transmitter. 
As will be discussed in section 3.5.3, the modified SLM method can significantly reduce out-of-band radiation even more effectively than for OFDM for the same back-off value. We will show that the efficiency of both methods is poor when the amplifier is more nonlinear up to the saturation point. To improve the SLM performance for a more nonlinear amplifier, we consider use of a memoryless predistortion technique to linearize the HPA characteristics. This technique has already been used for OFDM systems [11][43]. Since there are no other references about out-of-band radiation reduction of SM, we are not able to compare our results with that of other methods. Nevertheless we compare them with OFDM results.

As we show in Chapter 3, a similar question might arise about the PAPR of SM-CDMA and Multi Carrier-CDMA (MC-CDMA) systems. As a minor contribution, we investigate the PAPR of SM-CDMA in section 3.6 for different spreading methods and different constellations.

\subsubsection{Synchronization}

SM is known to be less sensitive than OFDM to frequency offset and phase noise [35][44]. In SM the phase error of consequent symbols within a block are highly correlated. In OFDM phase error causes inter-symbol interference after taking the FFT in the receiver. We discuss frequency offset and phase noise effects on SM systems with turbo equalization in section 2.6, and in more detail in Chapter 5. 


\subsection{Equalization methods for SM}

As mentioned in section 2.4.1, performing the equalization process in the frequency domain is computationally efficient. In this section we briefly review linear, decision feedback and turbo equalizers for SM systems. The block diagram of the first two methods are depicted in Figure 2-4 [14].

\subsubsection{Linear Frequency Domain Equalization}

The time domain output $\left(z_{n}\right)$ of a linear equalizer implemented in the frequency domain would be:

$$
z_{n}=\frac{1}{N} \sum_{k=1}^{N} W_{k} R_{k} \exp \left(j \frac{2 \pi(k-1)(n-1)}{N}\right), n=1, \ldots, N
$$

where $N$ is block length, $W_{k}$ and $R_{k}$ are the $k$-th filter coefficient and the $k$-th element of the received signal in the frequency domain. The error signal between the equalizer output and the transmitted data $\left(x_{n}\right)$ is:

$$
e_{n}=z_{n}-x_{n}
$$

The equalizer coefficients minimizing mean squared error, $J=E\left(\left|e_{n}\right|^{2}\right)$, are as follows [13]:

$$
W_{k}=\frac{H_{k}^{*}}{\left|H_{k}\right|^{2}+\sigma^{2}}, k=1, \ldots, N
$$

where $\sigma^{2}$ is the Gaussian noise variance and $H_{k}$ is the $k$-th element of channel frequency response. 


\subsubsection{Decision Feedback Equalization (DFE)}

We can also use DFE to achieve better performance at the expense of more complexity. In DFE we can implement the feed-forward filter in the frequency domain and the feedback coefficients in the time domain. The equalizer output is:

$$
z_{n}=\frac{1}{N} \sum_{k=1}^{N} W_{k} R_{k} \exp \left(j \frac{2 \pi(k-1)(n-1)}{N}\right)-\sum_{k \in F_{B}} f_{k}^{*} a_{m-k}, n=1, \ldots, N,
$$

where $\left\{f_{k}\right\}$ are the feedback filter coefficients. The filter coefficients, determined based on minimum mean squared error (MMSE) rule, are as follows [14]:

$$
\begin{gathered}
W_{k}=\frac{H_{k}^{*}\left(1+\sum_{l \in F_{B}} f_{l}^{*} \exp \left(-j \frac{2 \pi(k-1) l}{N}\right)\right)}{\left|H_{k}\right|^{2}+\sigma^{2}}, k=1, \ldots, N, \\
\underline{f}=-Q^{-1} \underline{q},
\end{gathered}
$$

where

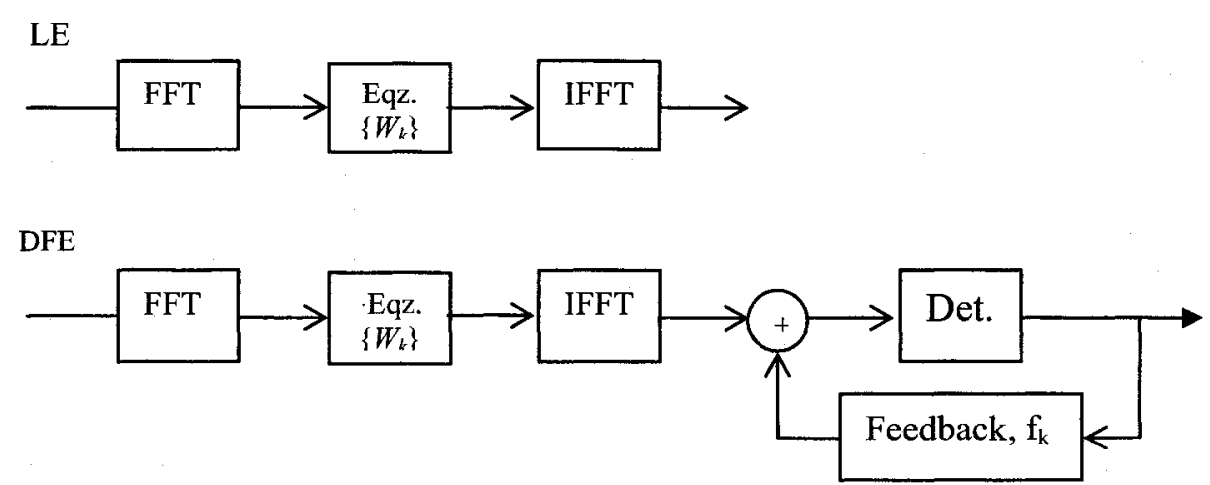

Figure 2-4. Block diagrams of Linear and Decision Feedback Equalizers. 


$$
\underline{q}=\left[\begin{array}{l}
q_{k_{1}} \\
\vdots \\
q_{k_{B}}
\end{array}\right], Q=\left[\begin{array}{lrr}
q_{0} & q_{k_{1}-k_{2}} \ldots & q_{k_{1}-k_{B}} \\
q_{k_{2}-k_{1}} & q_{0} \ldots & q_{k_{2}-k_{B}} \\
\vdots & & \\
q_{k_{B}-k_{1}} & q_{k_{B}-k_{2}} \cdots & q_{0}
\end{array}\right], q_{k}=\frac{\sigma^{2}}{N} \sum_{n=1}^{N} \frac{\exp \left(-j \frac{2 \pi(n-1) k}{N}\right)}{\left|H_{n}\right|^{2}+\sigma^{2}}
$$

\subsubsection{Turbo Frequency Domain Equalization (TFDE)}

One of the goals of this thesis is to propose an efficient equalization method for SM with reasonable complexity. In sections 2.5 .1 and 2.5 .2 , we briefly reviewed linear and decision feedback and equalizers with performance exceeding that of linear and DFE. In conventional systems using these equalizers, either in time or frequency domain, the equalization and decoding are two disjoint processes. To achieve better performance, an iterative equalization method known as turbo equalization was proposed in [45]. In this method, during iterations the equalizer uses the information generated by the decoder to improve the equalizer performance. The equalizer used in [45] is the complex but efficient MAP equalizer. Interference cancellation using linear equalizer has been suggested to reduce the system complexity [46]. Like other equalization systems, performing the equalization process in the frequency domain can make the system even less complex [47][48][49]. 


\subsubsection{System model}

In Chapter 3 we show the efficiency of SM for uplink transmission. In chapters 4, 5 and 6 we consider TFDE as the SM receiver whose transmitter and receiver block diagram is presented in Figure 2-5 and Figure 2-6 respectively. The details of these figures will be discussed in Chapter 4.

In this thesis we consider different schemes for equalizer and decoder in a TFDE. In [47][48] a non-adaptive TFDE is proposed in which the equalizer is fixed during iterations, independent of the decoder decisions. In Chapter 4, in addition to this case, we derive the equalizer coefficients with respect to the decoder outputs for an adaptive TFDE. Similar approach based on minimizing average mean squared error can be found in [76]. Therefore the equalizer coefficients change as iterations continue. Simulation results confirm the better performance of the adaptive design which show at least $1 \mathrm{~dB}$ improvement at BER of $5 \times 10^{-5}$.

For the decoder part, we compare the performance and complexity of TFDE with a low density parity check (LDPC) code, which is recently proposed to be used in the turbo equalizer [50][51][52], and with a convolutional code, which is traditionally used in turbo equalization [47][45]. None of the previous references have provided a performance and complexity comparison for a turbo equalizer with these two schemes. In section 4.3 it is shown that TFDE with LDPC codes can achieve better performance than the one with convolutional codes with nearly the same complexity. 


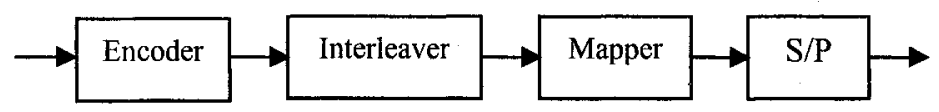

Figure 2-5. Transmitter model.

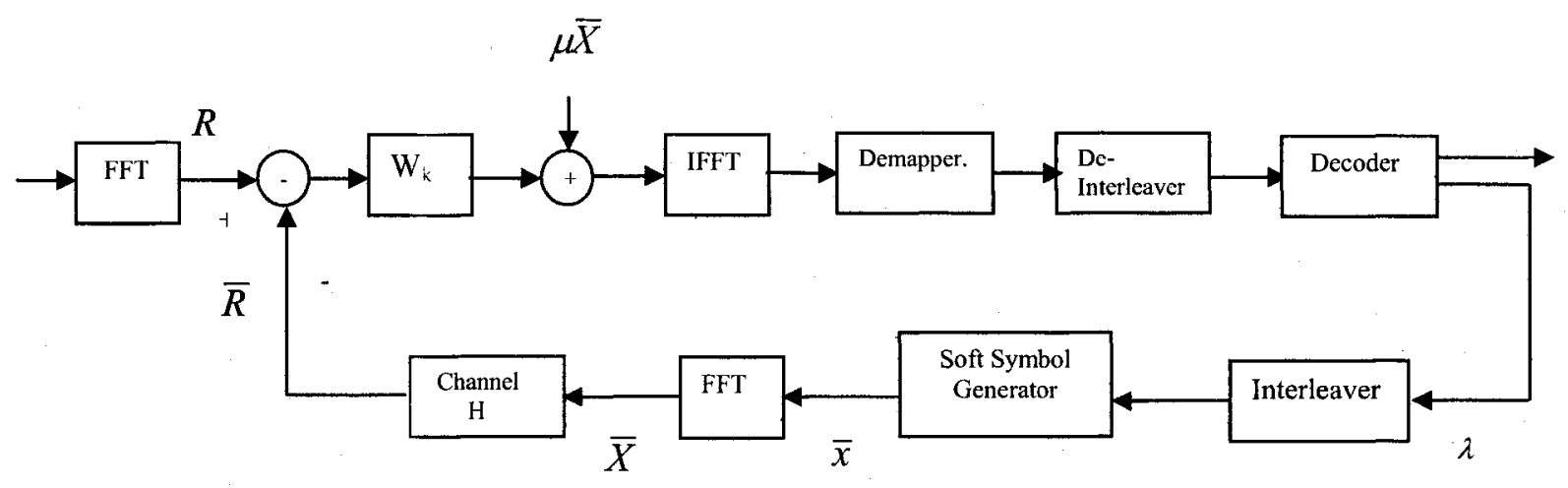

Figure 2-6. Receiver model (Turbo Frequency Domain Equalizer). 
The performance evaluation of turbo equalization requires extensive simulations. This process may become quite time consuming especially when comparison between alternate cases is performed and particularly in low bit error rate regions. An analysis tool which is generally used for iterative processes is the extrinsic information transfer (EXIT) chart, first proposed in [53][54]. This chart follows the extrinsic mutual information transferred between the two modules of the iterative process. This tool has been applied to turbo equalization in [55]. The EXIT chart is usually used to analyze the convergence of an iterative process. It can also be used for the asymptotic performance analysis when the block length approaches infinity. However it is not useful for performance prediction of a practical finite block length system. For such systems, the recently proposed BER transfer chart [56] can be used. The BERT chart is a tool similar to the EXIT chart in which instead of mutual information, the BER of each module is followed. In [56], in which this method is used for a turbo time domain equalizer, the equalizer BER curve is found inaccurately by interpolation between two extreme points. One of these points corresponds to the first iteration in which there is no a priori information available at the decoder. The second point corresponds to the case that we know the transmitted sequence perfectly, which does not happen in reality. In this thesis, for the first time the BERT chart is used for turbo frequency domain equalization. Here we propose a novel BERT chart method for TFDE in which we analytically find all points of the equalizer BER curve. The proposed BERT chart permits us to analyze a TFDE system with a finite block length. The simulation results confirm the accuracy of this new BERT chart method. 


\subsection{Synchronization in SM with TFDE}

Although the sensitivity of SM to frequency offset is less than that of OFDM, the synchronization for SM with turbo equalization has been the subject of quite a few papers; however none of them considered TFDE systems. In [57] only the frequency offset is considered for an iterative block decision feedback equalizer (IBDFE). IBDFE is an SM system with iterative frequency domain equalization while the decoder is not a part of the iterative loop. In [58] the time domain recovery has been performed for a turbo equalizer with time domain equalization. In this thesis we consider the effect of both frequency offset and phase noise for TFDE and we propose blind joint synchronization and turbo equalization methods. For frequency offset compensation, we suggest two methods based on the maximum likelihood criterion and computing the normalized correlation between the two halves of the block. In the presence of both phase noise and frequency offset, a decision directed phase lock loop method based on MAP criterion is suggested. In all these methods, the estimator takes advantage of the decoder output information to improve its estimation of the phase error. 


\section{Chapter 3 PAPR of OFDM and SM signals}

In this chapter we investigate the effect of high power amplifiers with nonlinear characteristics on the performance and spectrum of SM systems. We compare the results with those of OFDM. To reduce the out-of-band radiation of SM signals we propose a modified version of selected mapping algorithm and compare its structure and performance with the original SLM method for OFDM. To achieve the best performance of the proposed SLM method, we combine it with an ideal predistortion technique generally used for linearization of power amplifier characteristics. We also compare the effect of the constellation and spreading method on the out-of-band radiation and amplitude distribution of MC-CDMA and SM-CDMA. 


\subsection{Large Peak to Average Power Ratio problem}

Peak to average power ratio as presented in (3-1) is the ratio of maximum of instantaneous power to the average power of a signal,

$$
P A P R=\frac{\max _{0<t<T} P(t)}{P_{a v}},
$$

where $P(t)$ and $P_{a v}$ are the instantaneous and average power of the signal defined over a specified time interval, for instance the block duration. Signals with large PAPR suffer from the distortion generated by a nonlinear HPA. The HPA nonlinearity can cause performance degradation, in-band and out-of-band distortion unless the power amplifier has a sufficiently large back-off value [8]. On the other hand, to enhance the power efficiency and decrease the system cost, HPAs with smaller back-off are desirable.

Large PAPR is known as the most important drawback of OFDM. Signals having large PAPR are generated when the modulated complex symbols add together constructively in the IFFT operation. The SM method is equivalent to a DFT pre-coded OFDM [13]. The PAPR of the SM signal grows after filtering and Digital to Analog (D/A) conversion, with high peak values after filtering typically being associated with the occurrence of several successive large-amplitude constellation points. The most significant benefit of SM compared to OFDM is its lower envelope fluctuations and lower PAPR.

To compare the envelope distribution of OFDM and SM, we can look at their complementary cumulative distribution function (CCDF) which is depicted in Figure 3-1. As expected, high peaks in SM are less probable to occur than in OFDM. 


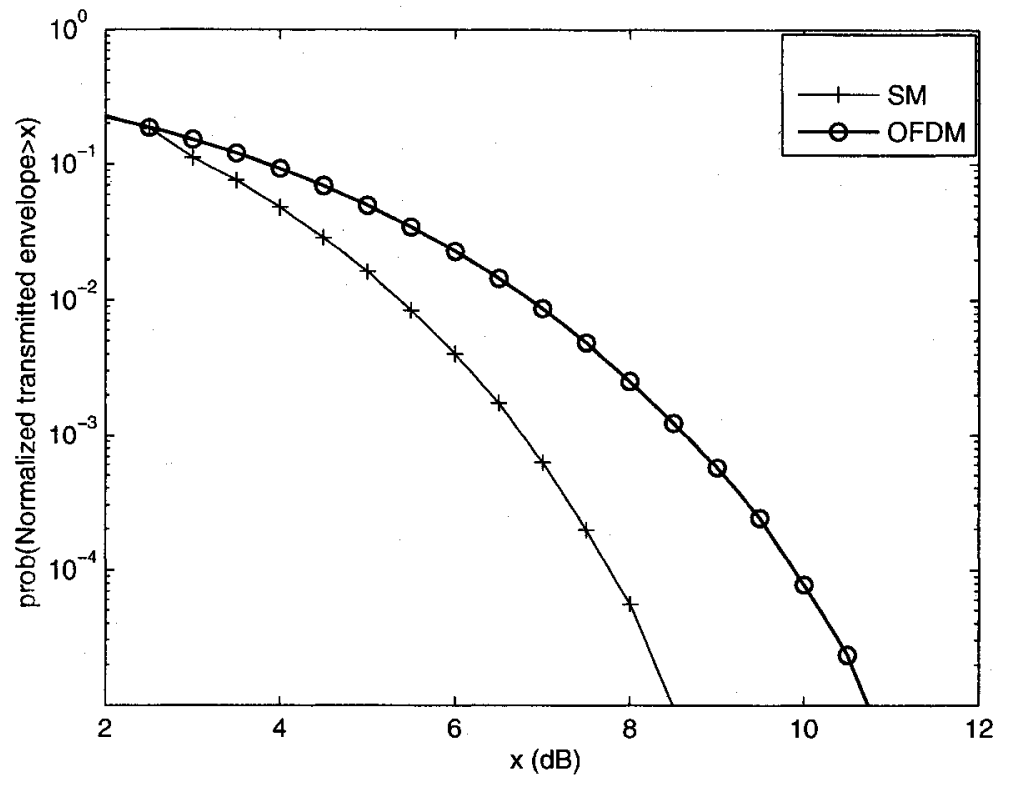

Figure 3-1. CCDF of OFDM and SM, 16-QAM constellation, $N=256$, rectangular frequency domain windowing, raised cosine time domain windowing $\left(\alpha_{t}=0.125\right)$.

\subsection{Amplifier model}

The amplifier characteristics are determined by Amplitude Modulation/Amplitude Modulation (AM/AM) and Amplitude Modulation /Phase Modulation (AM/PM) curves presenting the relationship between the amplifier input and output signals. Let $v_{i}(t)$ and $v_{o}(t)$ show the input and output signals of the amplifier and $F_{A}($.$) and F_{\Phi}($.$) present$ $\mathrm{AM} / \mathrm{AM}$ and AM/PM characteristics. If $V(t)$ and $\widetilde{\varphi}(t)$ are the amplitude and phase of the input signa!, we have:

$$
\begin{gathered}
v_{i}(t)=V(t) \exp \left(j\left[2 \pi f_{c} t+\widetilde{\varphi}(t)\right]\right), \\
v_{o}(t)=F_{A}(V(t)) \exp \left(j\left[2 \pi f_{c} t+\widetilde{\varphi}(t)+F_{\Phi}(V(t))\right]\right) .
\end{gathered}
$$

An ideal amplifier does not add non-uniform phase shift to the signal and its AM/AM curve is linear. The Solid State Power Amplifier (SSPA) is a commonly used amplifier in 
wireless portable communication systems. The model generally used for SSPA is an AM/AM model known as the Rapp model [59]. However, the best model for each amplifier can be found by measurement. The Rapp AM/AM model is as follows:

$$
F_{A}(V)=\frac{V}{\left(1+\left(\frac{|V|}{V_{s}}\right)^{2 p_{0}}\right)^{\frac{1}{2 p_{0}}}} .
$$

The Rapp model is identified by two parameters, the saturation level $\left(V_{s}\right)$ and a factor $p_{0}$ determining the smoothness of the AM/AM curve. As presented in Figure 3-2, for large $p_{0}$ the amplifier is linear up to the saturation level and behaves like a clipper while for small $p_{0}$ the signal is more nonlinearly amplified and goes to the saturation level gradually.

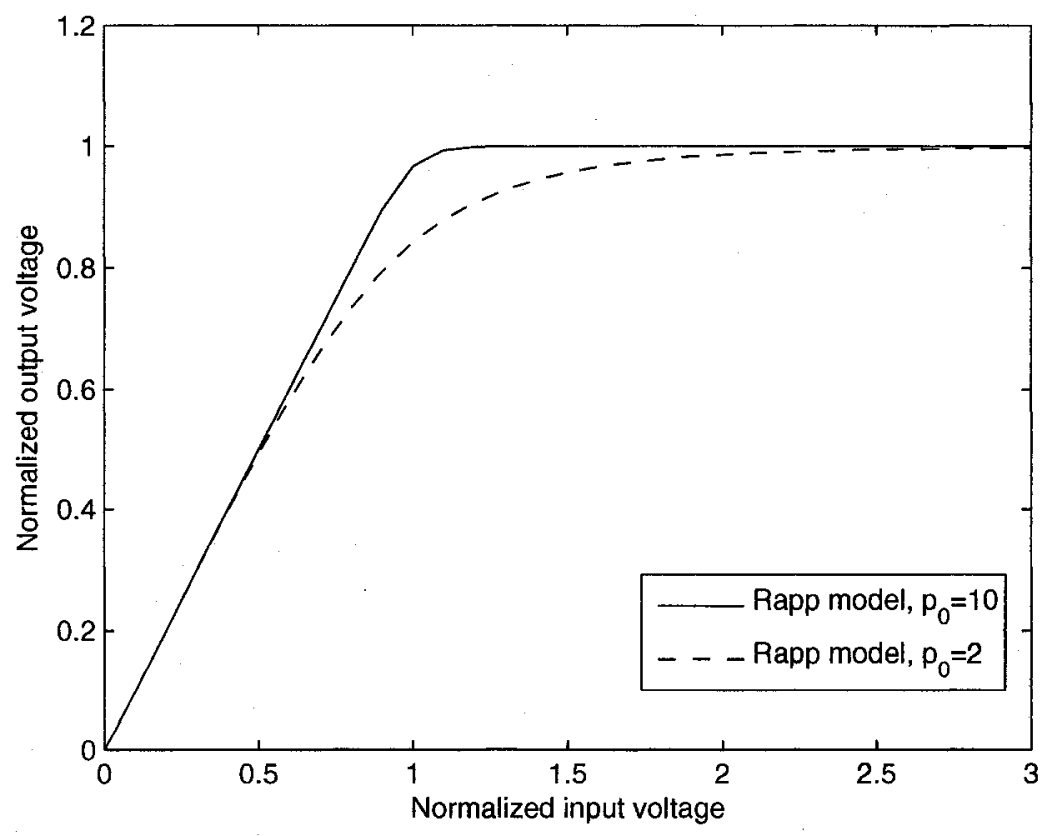

Figure 3-2. AM/AM curve of Rapp model. 
As will be seen later this effect makes the out-of-band radiation power of amplifiers with smaller $p_{0}$ to be more concentrated at lower frequencies.

In [60] a modified Rapp model based on the measurements of a bipolar SSPA was suggested which considers the AM/PM curve. The Saleh model is another model suggested to specify the characteristics of a nonlinear amplifier [61]. It was originally suggested to model the behavior of Traveling Wave Tube (TWT) amplifiers and can accurately model this type of amplifier. Although by modification of the Saleh model parameters it can also be used for SSPA, the accuracy decreases especially for the AM/AM curve. Another model which is customized to a Ga-As FET SSPA and matches the measurements is the Ghorbani model [62]. The AM/PM of this model is similar to that of Saleh model while its AM/AM curve matches better than that of Saleh model to typical measured data. The AM/AM and AM/PM functions of Ghorbani model are as follows:

$$
F_{A}(V)=\frac{\hat{x}_{1} V^{\hat{x}_{2}}}{1+\hat{x}_{3} V^{\bar{x}_{2}}}+\hat{x}_{4} V, F_{\Phi}(V) \cong \frac{\hat{y}_{1} V^{\hat{y}_{2}}}{1+\hat{y}_{3} V^{\bar{y}_{2}}}+\hat{y}_{4} V .
$$

The parameters of this model are as follows:

$$
\begin{aligned}
& \widehat{x}_{1}=8.1081, \widehat{x}_{2}=1.5413, \widehat{x}_{3}=6.5202, \widehat{x}_{4}=-0.0718, \\
& \hat{y}_{1}=4.6645, \hat{y}_{2}=2.0965, \hat{y}_{3}=10.88, \hat{y}_{4}=-0.003 .
\end{aligned}
$$

Figure 3-3 shows the AM/AM and AM/PM characteristics of the Ghorbani model. The most frequently used HPA model in the references is Rapp model. Therefore the primary model in this chapter is the Rapp model while in different cases we use the Ghorbani model for comparison. 


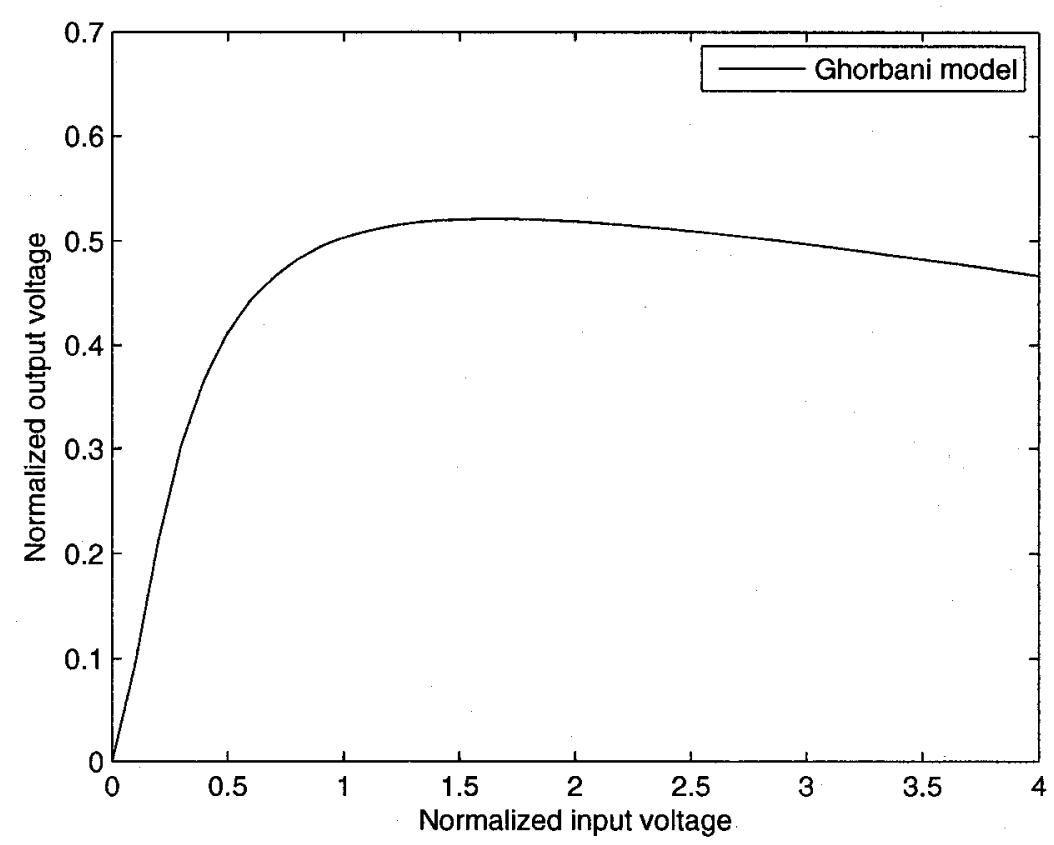

a) AM/AM curve of Ghorbani model

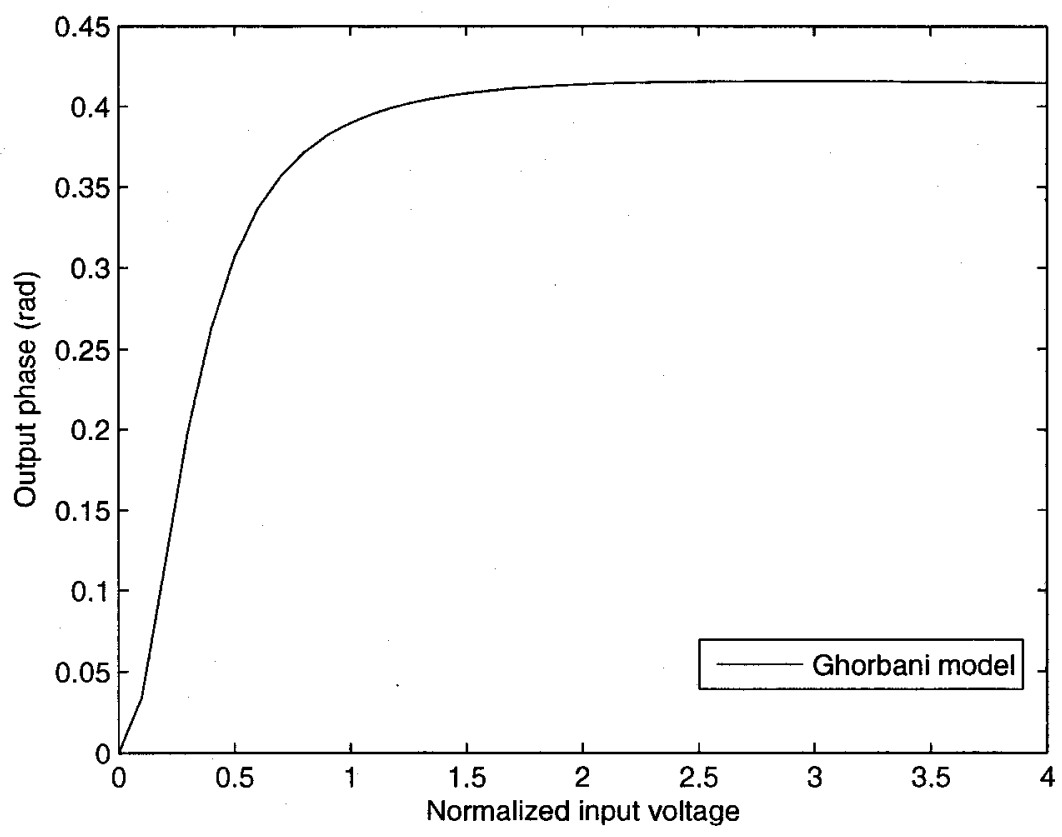

b) AM/PM curve of Ghorbani model

Figure 3-3. Ghorbani model for nonlinear amplifier. 
Input power Back-Off (BO) is another important characteristic of the amplifier which is defined as:

$$
\mathrm{BO}=10 \log \left(\frac{P_{s}}{\bar{P}_{i}}\right)
$$

where $P_{s}$ is the input power corresponding to the saturation level and $\bar{P}_{i}$ is the average input power. An amplifier needs larger power back-off to tolerate signals with large dynamic ranges. However for more power efficiency the amplifier should have a smaller power back-off value.

\subsection{Nonlinear amplifier effect on out-of-band radiation}

As mentioned earlier, the nonlinearity of the amplifier results in out-of-band radiation which interferes with the adjacent channels. In most communication systems, there is a standard power spectrum mask determining the maximum tolerable amount of out-ofband radiation. These spectrum masks are usually determined based on the system requirements like noise floor, adjacent channel power ratio and allocated bandwidth. Figure 3-4 illustrates one of these masks designed in the WINNER project for wideband (WB) and narrowband (NB) mode for bandwidths of 83.2 and $10.4 \mathrm{MHz}$ respectively. In this figure the power spectrum masks for both a mobile terminal (MT) and an access point (AP) are shown [63].

The out-of-band radiation depends on the signal dynamic range, the power back-off of the amplifier and the amount of AM/AM and AM/PM nonlinearity. For smaller back-off 
values for a fixed input power, the amplifier saturation level would be lower. This increases the number of clipped samples in a block which results in more distortion.

If an amplifier has small Rapp model parameter $p_{0}$, the out-of-band radiation has smaller components at higher frequencies, and most of the out-of-band power is concentrated close to the in-band spectrum. On the other hand, when $p_{0}$ is large and significant clipping occurs, the out-of-band radiation components are spread over a wider frequency range. It can be justified if we use the binomial expansion for the denominator of the Rapp model. The expansion of the Rapp model would be:

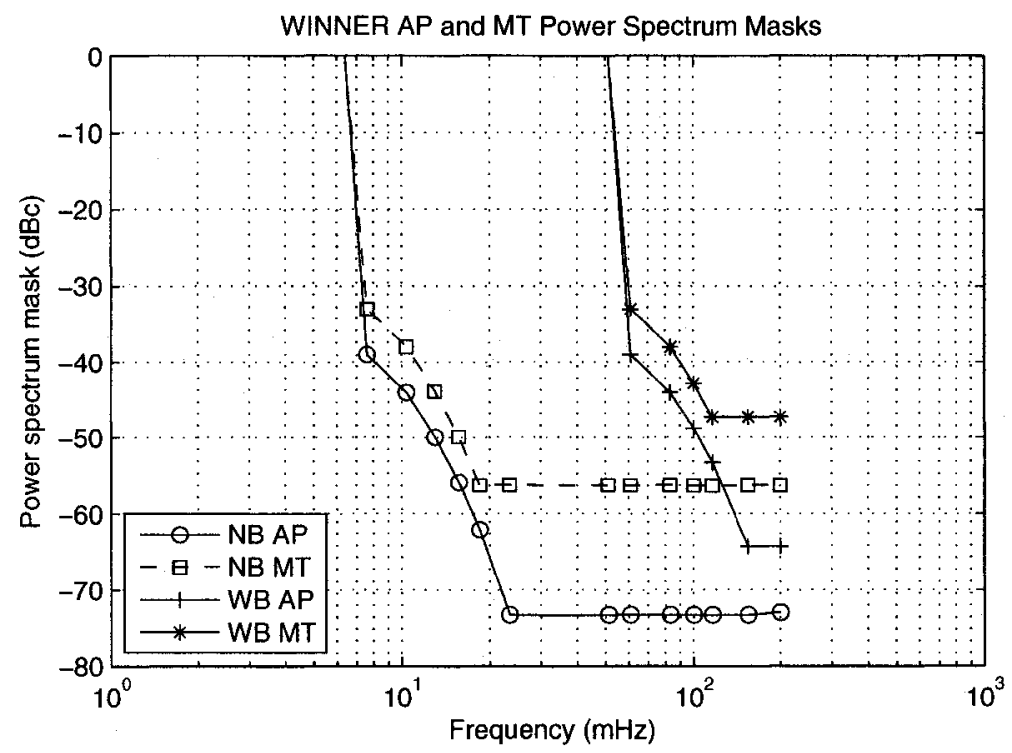

Figure 3-4. WINNER spectrum mask. 


$$
v_{o}(t)=\left\{\begin{array}{lc}
v_{i}(t)+\sum_{k=1}^{\infty} \bar{\alpha}_{k}\left[v_{i}(t)\right]^{2 p_{0} k+1} & v_{i}(t)<V_{s} \\
V_{s}+\sum_{k=1}^{\infty} \bar{\beta}_{k}\left[v_{i}(t)\right]^{-2 p_{0} k} & v_{i}(t)>V_{s}
\end{array},\right.
$$

where $\bar{\alpha}_{k}=(r)_{k} V_{s}^{-2 p_{0} k}, \bar{\beta}_{k}=(r)_{k} V_{s}^{2 p_{0} k}, r=\frac{-1}{2 p_{0}}$ and $(r)_{k}$ is the Pochhammer symbol defined as:

$$
(r)_{k} \stackrel{\Delta}{=} \frac{r(r-1) \ldots(r-k+1)}{k !} .
$$

If we assume that the saturation level is high enough to use only the first expression in (3-7) for $v_{i}<V_{s}$, and compare the out-of-band radiation of amplifiers with two different $p_{0}$ 's, the corresponding outputs $\left(v_{o}\right)$ for $p_{0}=2$ and $p_{0}=10$ would be:

$$
\begin{gathered}
v_{o, p_{0}=2}(t)=v_{i}(t)+(r)_{1} V_{s}^{-4}\left[v_{i}(t)\right]^{5}+(r)_{2} V_{s}^{-8}\left[v_{i}(t)\right]^{9}+\ldots, \\
v_{o, p_{0}=10}(t)=v_{i}(t)+\left(r^{\prime}\right)_{1} V_{s}^{-20}\left[v_{i}(t)\right]^{21}+\left(r^{\prime}\right)_{2} V_{s}^{-40}\left[v_{i}(t)\right]^{41}+\ldots,
\end{gathered}
$$

where $r=-\frac{1}{4}$ and $r^{\prime}=-\frac{1}{20}$. As can be seen, the expansion of the output for $p_{0}=2$ includes smaller powers of the input signal. For instance there are $v_{i}^{5}, v_{i}^{9}, v_{i}^{13}, v_{i}^{17}$ before the common component $v_{i}^{21}$. The $m$-th power of the input signal in time domain is equivalent to the $m$ times convolution of the signal Fourier transform by itself in the frequency domain. Roughly speaking the resulting bandwidth would be $m$ times the original bandwidth. This fact confirms that for $p_{0}=2$, the out-of-band radiation power is more concentrated in frequencies closer to the in-band spectrum. The second terms in 
(3-9) and (3-10) expansions generate the most powerful part of the distortion. Thus we can ignore other terms compared to this one. For $p_{0}=2$, this term is larger than that of $p_{0}=10$. This makes the out-of-band radiation of the amplifier with $p_{0}=2$ more powerful than that of an amplifier with $p_{0}=10$. Figure 3-5 and Figure 3-6 depict the outof-band radiation of SM and OFDM with 16-QAM constellation for the Rapp model with $p_{0}=10$ and $p_{0}=2$ respectively. Figure 3-7 shows the out-of-band radiation when the Ghorbani model is used. In the simulations, the transmitted blocks $\left(\mathbf{a}=\left[a_{0} \ldots a_{N-1}\right]\right)$ contain $N$ symbols selected from a 16-QAM constellation. To generate the over-sampled (with rate $M$ ) transmitted signal, we follow these steps:

- Take the FFT of the original block; $\mathbf{A}=F F T(\mathbf{a})$.

- Generate the block $\mathbf{B}$ by $M$ times repeating $\mathbf{A} ; \mathbf{B}_{1 \times M N}=[\mathbf{A} \ldots \mathbf{A}]$.

- Use the frequency domain pulse shaping filter $\widetilde{\mathbf{B}}=\mathbf{B} \bullet \check{\mathbf{P}}$. (e.g., for zero excess bandwidth $\left.\breve{\mathbf{P}}=\left[\mathbf{1}_{1 \times N} \mathbf{0}_{1 \times(M-1) N}\right]\right)$.(The operator $\bullet$ is element by element multiplication of two vectors, $\mathbf{1}_{K \times L}$ and $\mathbf{0}_{K \times L}$ are all one and all zero $K \times L$ matrices respectively.)

- Take the IFFT of the signal, $\widetilde{\mathbf{b}}=\operatorname{IFFT}(\widetilde{\mathbf{B}})$.

- Add cyclic prefix.

- Multiply by time domain window. 


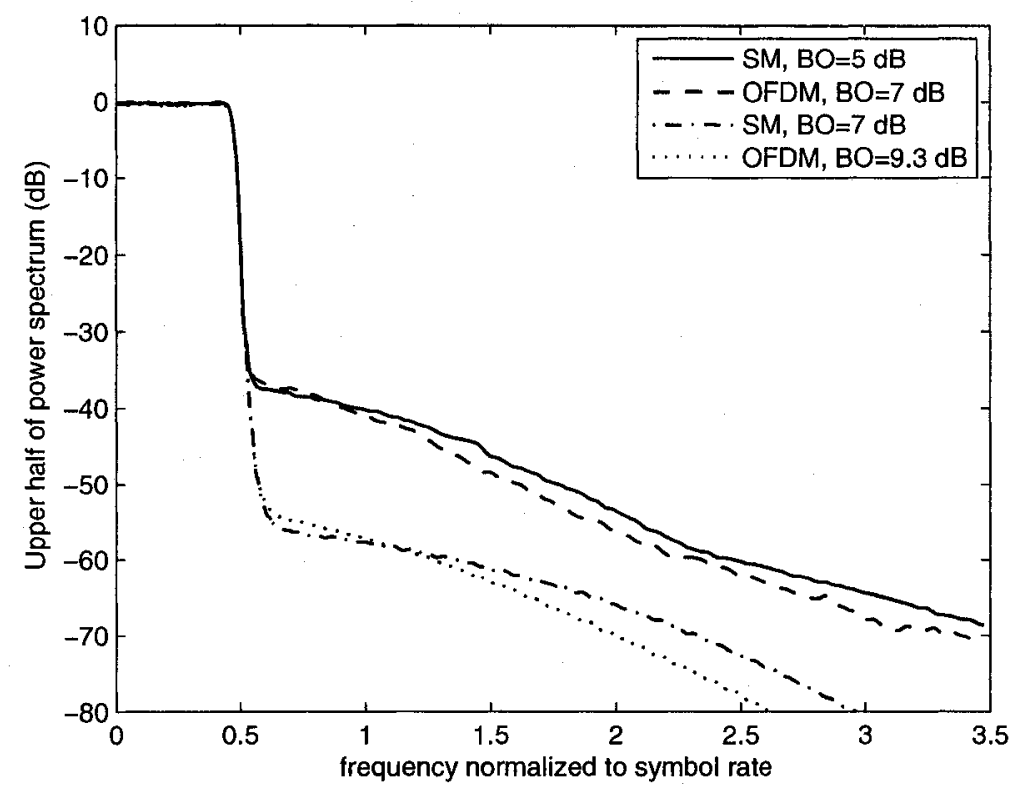

Figure 3-5. Out-of-band radiation of SM and OFDM for $p_{0}=10,16-\mathrm{QAM}$ constellation.

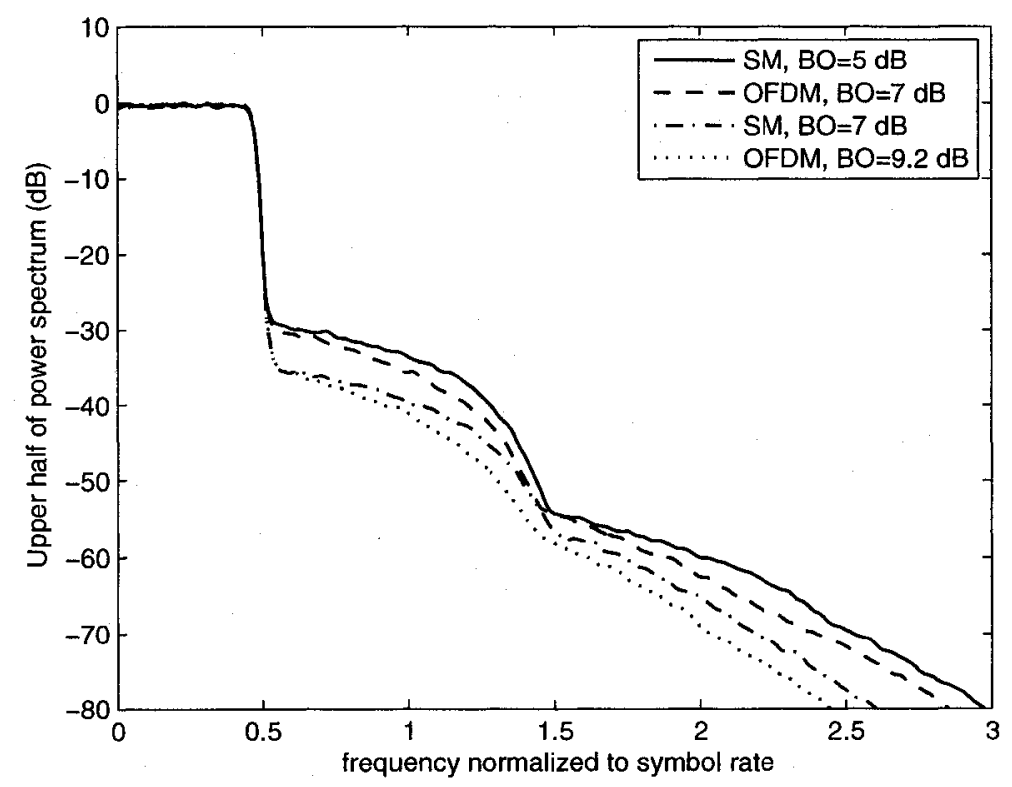

Figure 3-6. Out-of-band radiation of SM and OFDM for $p_{0}=2$, 16-QAM constellation. 


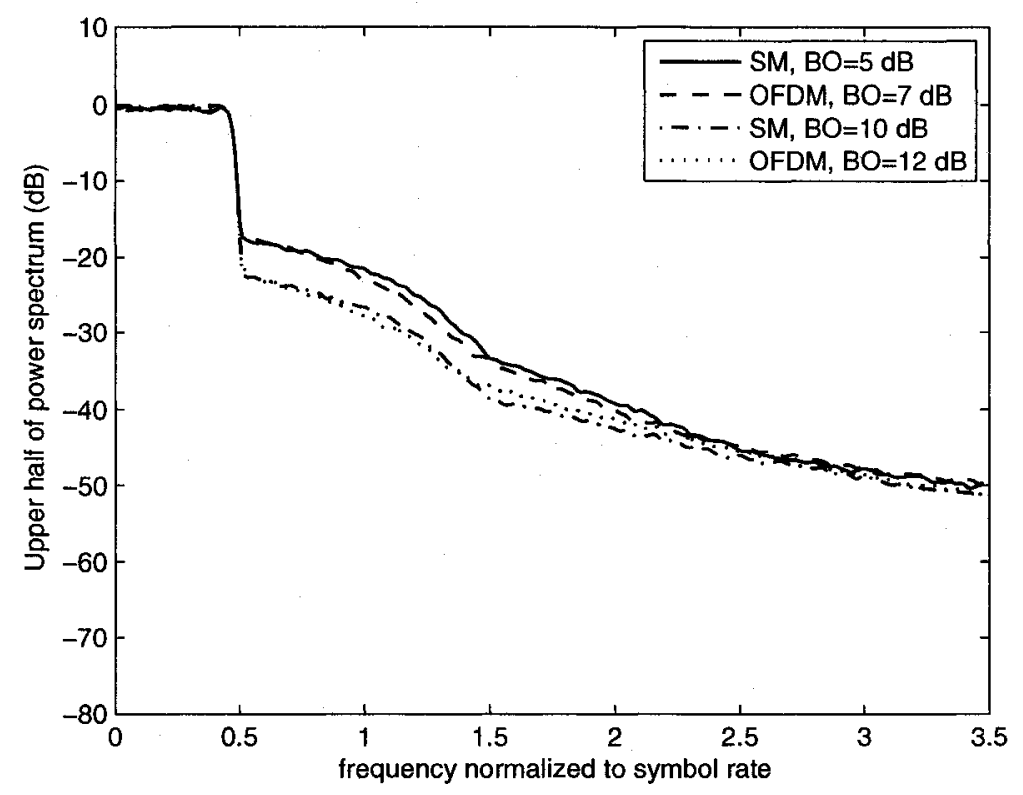

Figure 3-7. Out-of-band radiation of SM and OFDM for Ghorbani model, 16-QAM constellation.

To generate the curves in Figure 3-5, Figure 3-6 and Figure 3-7, we used (FFT length) $N=256$ and (over-sampling rate) $M=8$. We employed rectangular frequency domain windowing and raised cosine time windowing with roll-off factor of $\alpha_{t}=0.125$. For a SM signal we can reduce the out-of-band radiation ${ }^{3}$ at the expense of using some extra bandwidth by employing more efficient pulse shaping filters like a raised cosine filter with roll-off factor of $\alpha_{f}$ (which is equivalent to frequency domain windowing with a raised cosine window). This effect is presented in Figure 3-8 in which by using filters with $\alpha_{f}=0.1$ and $\alpha_{f}=0.2$ we need $0.6 \mathrm{~dB}$ and $1 \mathrm{~dB}$ less back-off value. In all these figures and similar ones in this chapter, what is depicted is relative power spectrum assuming $0 \mathrm{~dB}$ as the in-band Power Spectral Density (PSD).

\footnotetext{
${ }^{3}$ Or equivalently we can reduce the required back-off for the same PSD.
} 
In Figure 3-5 and Figure 3-6 the PSD of SM signal is presented for back-offs of 5 and 7 dB. In Figure 3-7 the back-off for the SM is 5 and $10 \mathrm{~dB}$. For each SM case we present the PSD of OFDM for a back-off value such that we achieve a PSD similar to that of SM. In this case, the OFDM requires 2 to $2.3 \mathrm{~dB}$ larger BO than that of SM. As discussed above, amplifiers with smaller back-off values and those which are more nonlinear up to the saturation level (smaller $p_{0}$ in Rapp model) generate more out-of-band radiation. Since the nonlinearity of Ghorbani model is more than that of Rapp model, the corresponding out-of-band radiation is larger too.

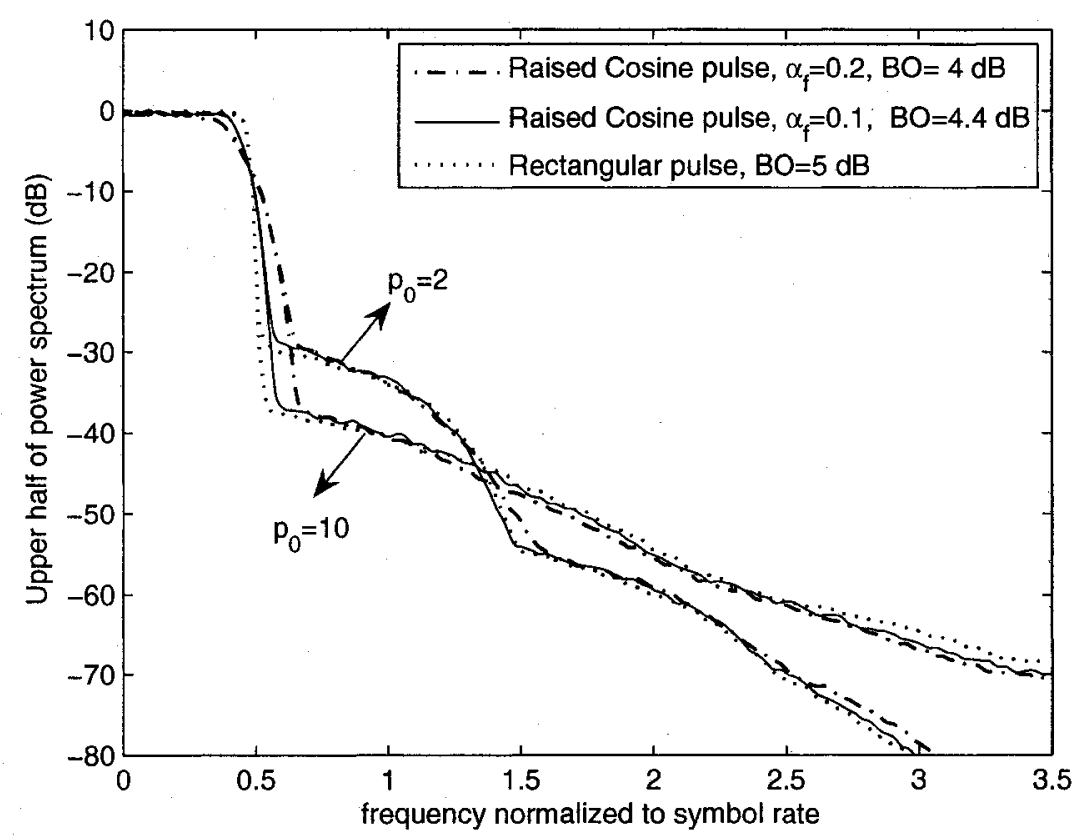

Figure 3-8. Out-of-band radiation of SM with different frequency domain windowing, 16-QAM constellation. 


\subsection{Nonlinear amplifier effect on the BER performance}

For the same back-off, the performance degradation generated by the nonlinear amplifier for OFDM is more significant than for SM due to the larger portion of the signal being clipped by HPA in OFDM. We can verify this claim explicitly by comparing the scatter diagram of both signals with 16-QAM at the transmitter output presented in Figure 3-9 and Figure 3-10. The nonlinear amplifier effect on the SM and OFDM performance in Additive White Gaussian Noise (AWGN) channel is depicted in Figure 3-11 and Figure 3-12 for $p_{0}=2$ and in Figure 3-13 and Figure 3-14 for $p_{0}=10$. As expected, performance degradation of OFDM is larger than that of SM. When the amplifier is linear up to the saturation level $\left(p_{0}=10\right)$, there is no performance degradation for SM even for a back-off of $3 \mathrm{~dB}$ while OFDM suffers from performance loss although it is less than the corresponding amount for $p_{0}=2$. For an amplifier with $p_{0}=2$, the degradation is not negligible even for SM for instance it is about $2 \mathrm{~dB}$ and $1.5 \mathrm{~dB}$ for back-off of $3 \mathrm{~dB}$ and $4 \mathrm{~dB}$ to achieve $\mathrm{BER}$ of $6 \times 10^{-4}$. It is worthwhile to mention that for typical spectral masks like those of Figure 3-4, the back-off values required for the HPA output spectrum to comply with the mask are large enough that the nonlinear distortion effect on performance is negligible. 


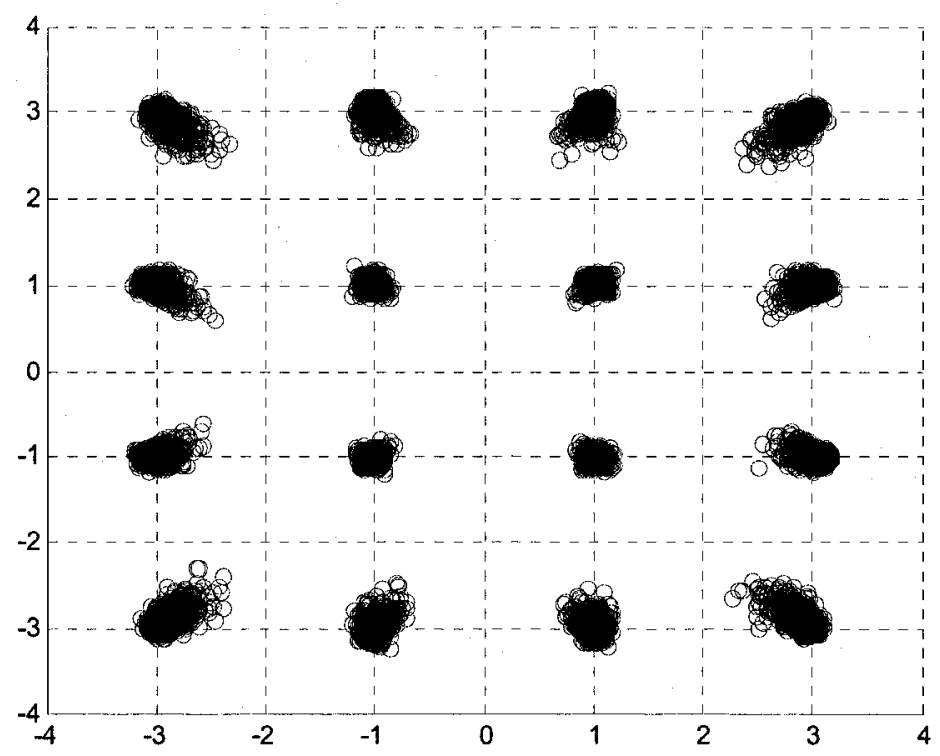

Figure 3-9. Scatter diagram of $\mathbf{S M}$ at the transmitter output, $p_{0}=2, \mathbf{B O}=\mathbf{5} \mathbf{d B}$.

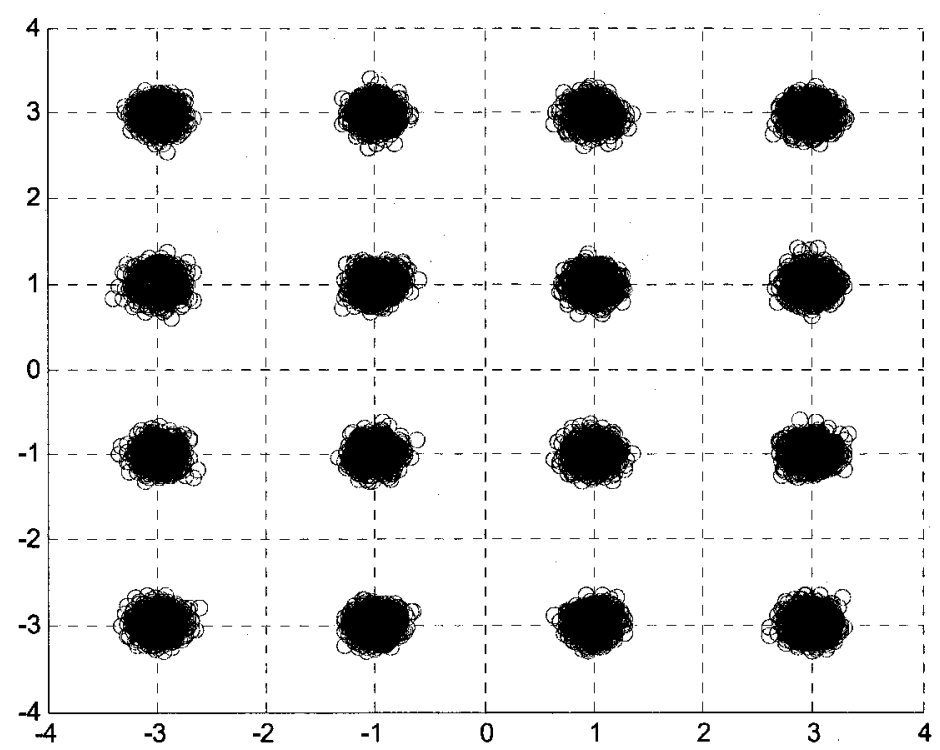

Figure 3-10. Scatter diagram of $\mathrm{OFDM}$ at the transmitter output, $p_{0}=2, \mathrm{BO}=7 \mathrm{~dB}$. 


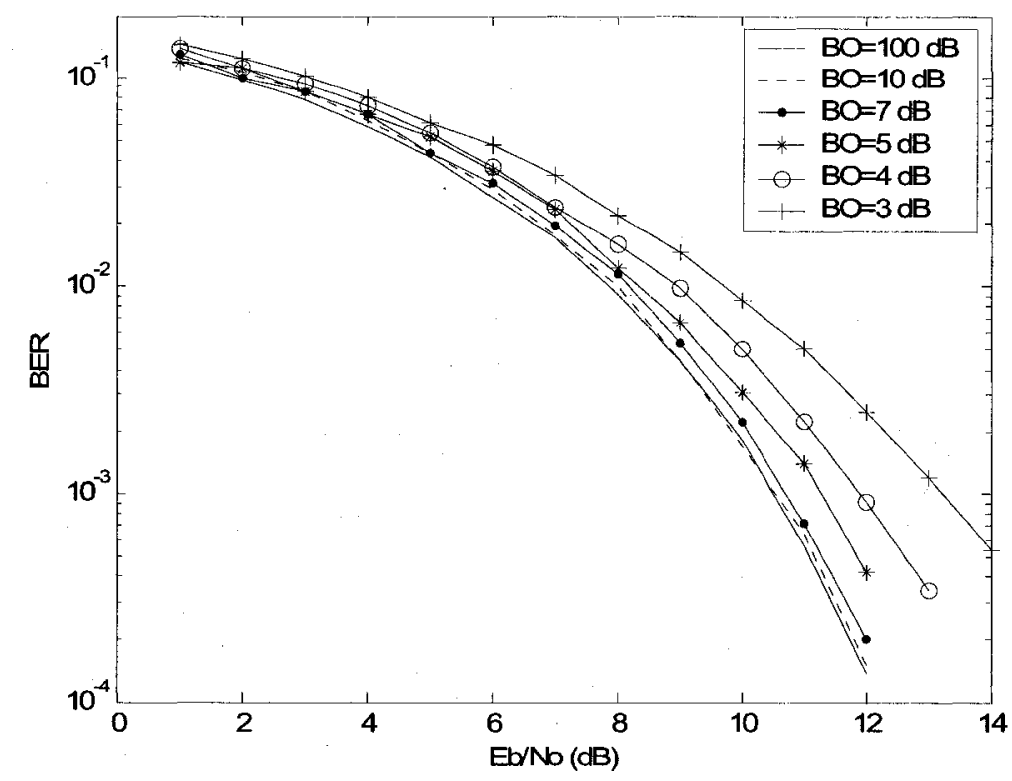

Figure 3-11. Effect of nonlinear amplification on BER of SM in AWGN, $p_{0}=2$, 16-QAM constellation.

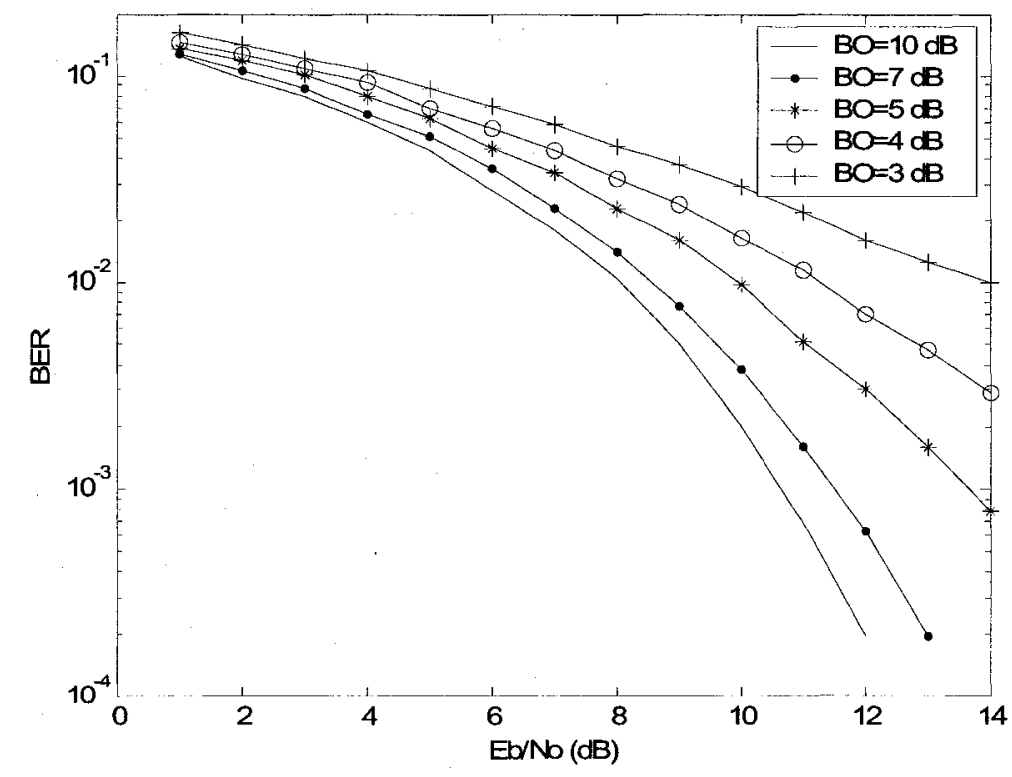

Figure 3-12. Effect of nonlinear amplification on BER of OFDM in AWGN, $p_{0}=2,16-\mathrm{QAM}$ constellation. 


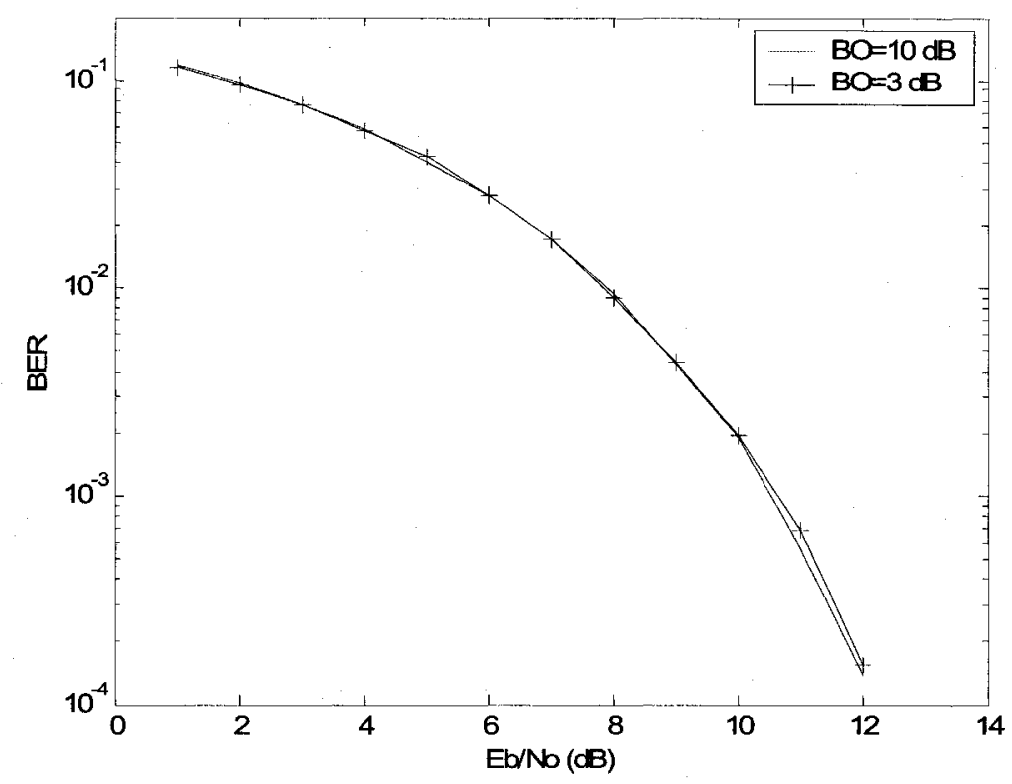

Figure 3-13. Effect of nonlinear amplification on BER of SM in AWGN, $p_{0}=10,16-$ QAM constellation.

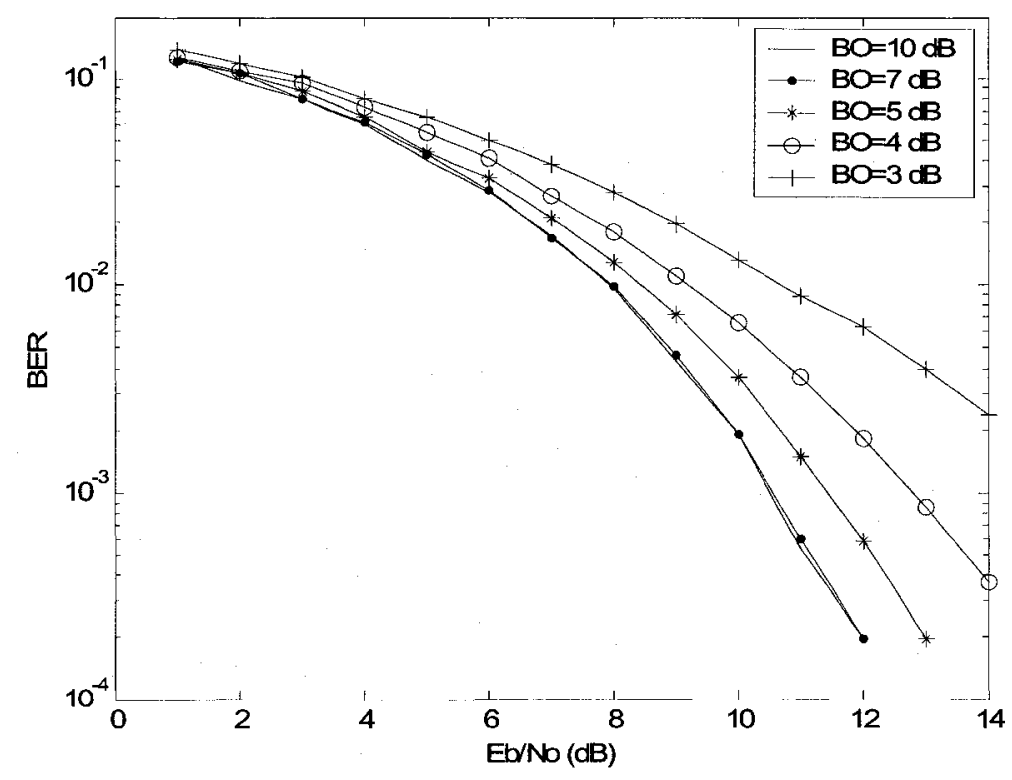

Figure 3-14. Effect of nonlinear amplification on BER of OFDM in AWGN, $p_{0}=10,16-\mathrm{QAM}$ constellation. 


\subsection{PAPR reduction methods}

Different methods have been proposed to reduce the PAPR of OFDM signals. The simplest and most straightforward one is clipping and filtering, in which the signal is clipped to some desirable level [8][31]. Some other PAPR reduction methods (e.g., coding method) are based on preventing the occurrence of OFDM symbols with large PAPR. In this approach only the OFDM symbols whose PAPR is below some determined level are transmitted [10][34]. Contrary to the clipping method, the coding method does not degrade the performance. The redundancy added to the signal is used for both PAPR reduction and error correction at the expense of requiring extra bandwidth. Partial Transmit Signaling (PTS) and Selected Mapping (SLM) are other PAPR reduction methods based on examining alternate signals for a generated data block [32][33]. In PTS the original block is divided into several sub-blocks where each one is multiplied by a

different coefficient. In the following sub-sections we propose a modified SLM algorithm to be used for SM. Since clipping is regarded as the basic PAPR reduction method, we briefly review this method first.

\subsubsection{Clipping method}

In the clipping method the transmitted signal, before amplification, is deliberately clipped to some desirable level. Therefore the amplifier input signal would be below the amplifier's saturation point. Clipping the signal generates performance degradation and out-of-band radiation. To eliminate the spectral regrowth, the signal should be filtered after clipping. The filtering can cause some undesirable peak regrowth [64]. The 
performance degradation would not be negligible especially when the clipping level is small. The benefit of this method compared to other PAPR reduction methods is the simplicity and not requiring extra bandwidth or extra receiver signal processing. By this method low out-of-band radiation can be achieved at the expense of some in-band distortion which degrades the system performance [8].

We can use the clipping and filtering method for SM to reduce out-of-band radiation. Figure 3-15 and Figure 3-16 show the effect of this method on the performance of SM when the clipping level equals to the amplifier saturation level. In Figure 3-15 and Figure 3-16 we observe some performance degradation compared to the corresponding curves in Figure 3-11 and Figure 3-13. This performance loss is generated in the filtering process which is performed after clipping to reduce the out-of-band radiation. In some cases different stages of clipping and filtering are used. However for the presented figures we used one stage of clipping and filtering.

\subsubsection{SLM method}

SLM is a recognized method for PAPR reduction in OFDM systems [32][33][65]. This method is based on generating $K$ different transformed blocks for a given data block and transmitting the one with the lowest PAPR. Some side information has to be transmitted to the receiver about the identity of the block transform. Hence the probability of high peak occurrence can be decreased. If the transformed sequences are statistically independent, the probability that the PAPR of all sequences exceeds a determined level $\left(L_{1}\right)$ is: 


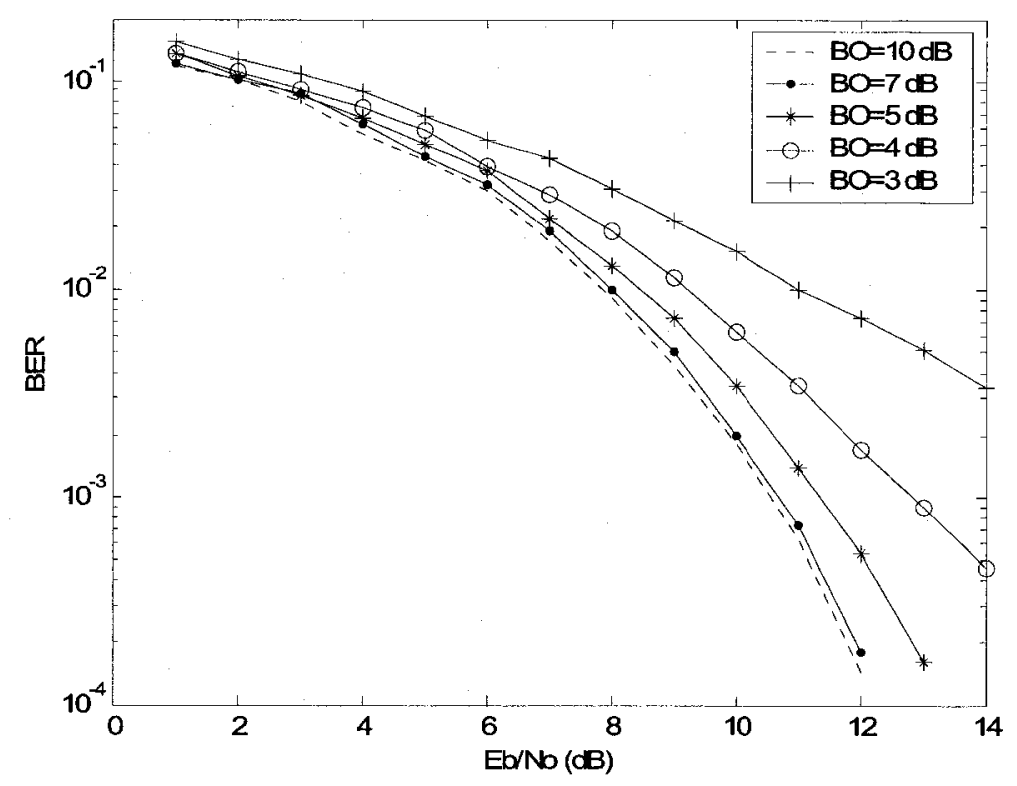

Figure 3-15. Effect of Clipping and Filtering on performance of SM, $p_{0}=2$

(the clipping level equals the amplifier saturation level).

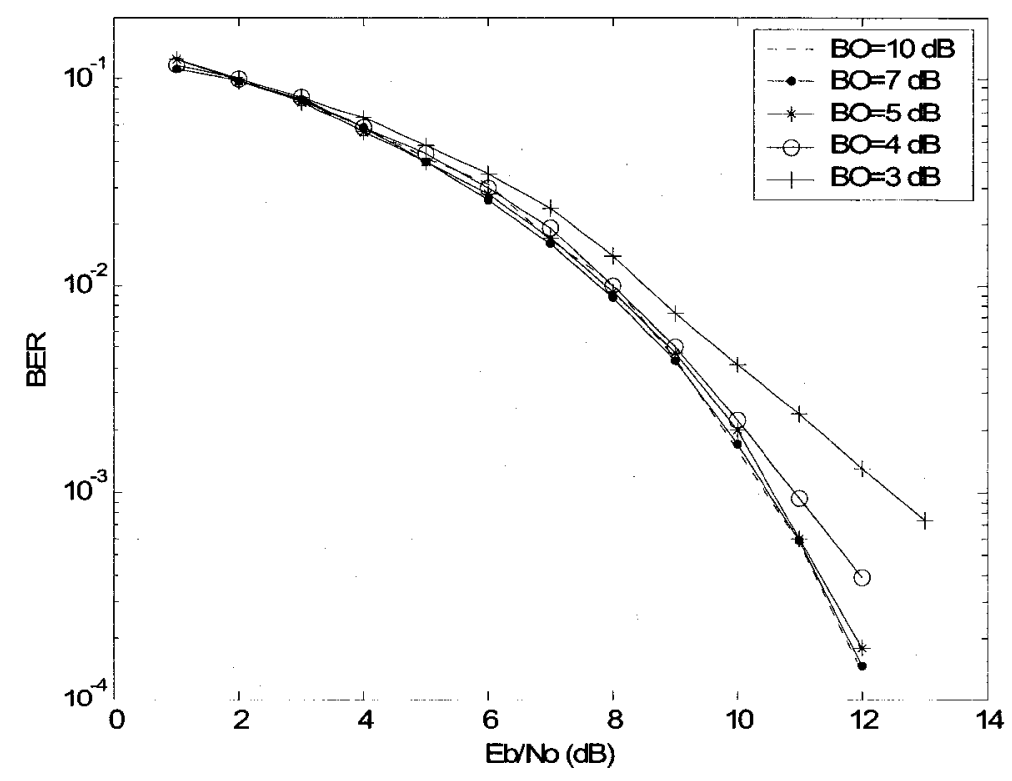

Figure 3-16. Effect of Clipping and Filtering on performance of SM, $p_{0}=10$

(the clipping level equals the amplifier saturation level). 
$\operatorname{Pr}\left\{P A P R_{1}>L_{1}, \ldots, P A P R_{K}>L_{1}\right\}=\prod_{i=1}^{K} \operatorname{Pr}\left\{P A P R_{i}>L_{1}\right\}=\left(\operatorname{Pr}\left\{P A P R_{1}>L_{1}\right\}\right)^{K}$

This probability is considerably smaller than the probability of the original signal PAPR exceeding the desired level $\left(\operatorname{Pr}\left\{P A P R_{1}>L_{1}\right\}\right)$.

Figure 3-17 shows the block diagram of this method for OFDM. To generate independent data blocks, each block is multiplied symbol-by-symbol, before the IFFT operation, by one of pseudo-random but fixed set of vectors $r_{i}$ whose elements are complex numbers with unit amplitude and a random phase uniformly distributed between $[0,2 \pi]$. The identity of the vector $r_{i}^{\prime}$ should be transmitted to the receiver as side information. The improvement achieved by SLM method in reducing PAPR of OFDM is presented in Figure 3-18. The probability that normalized transmitted envelope exceeds 9 $\mathrm{dB}$ is $6 \times 10^{-4}$ in the original OFDM signal. By using the SLM method with $K=4$ we are able to reduce this probability to $3 \times 10^{-6}$.

\subsubsection{Modified SLM method}

In OFDM, high peaks are generated in the IFFT operation. In SM systems high peaks are generated after filtering and D/A conversion and are usually associated with consecutive large magnitude symbols in the original data sequence. Consequently, the number of high local peaks in a SM block is larger than that of OFDM. This makes the distribution of the amplitude of SM different from that of OFDM. Here we suggest a modified version of the SLM algorithm for SM, designed based on the inherent differences between the cause of high peaks in OFDM and SM. 


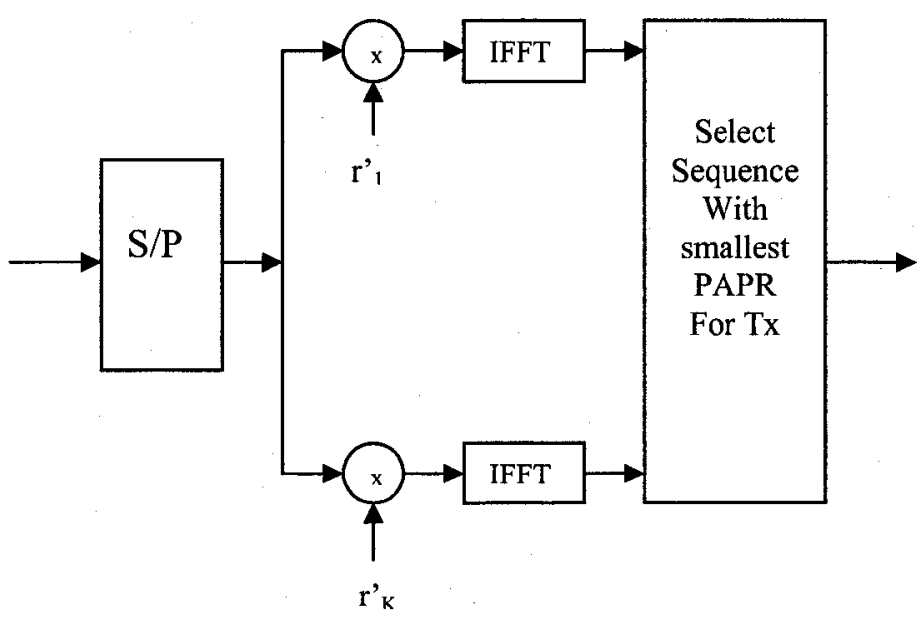

Figure 3-17. Block diagram of conventional SLM method ([32]).

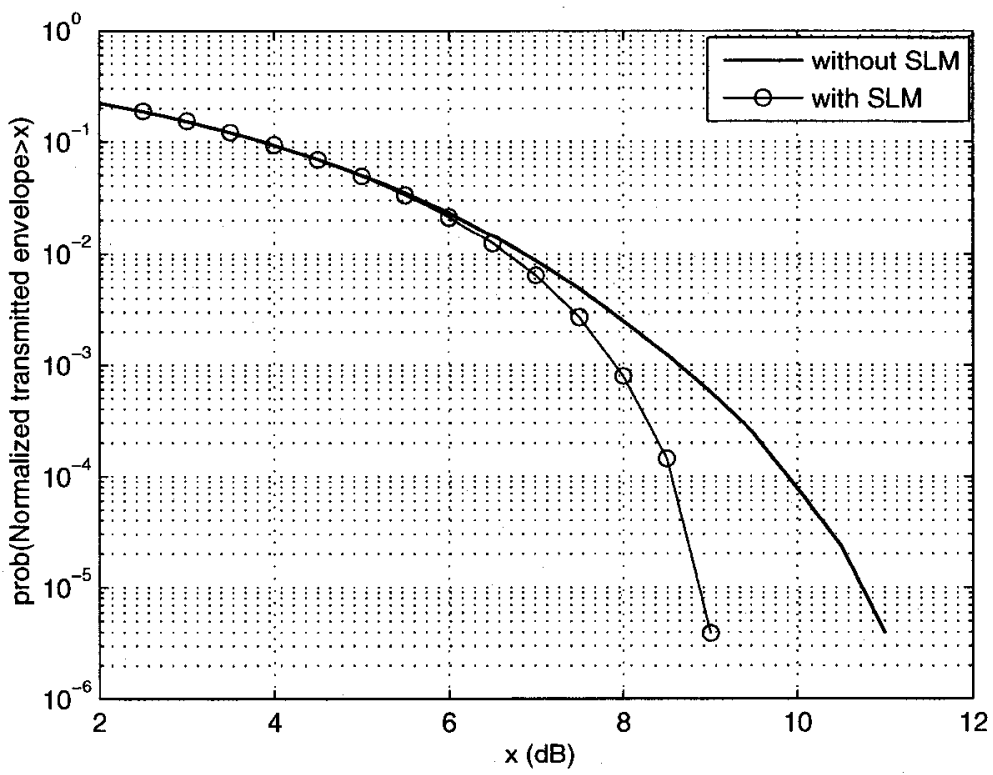

Figure 3-18. SLM effect on CCDF of amplitude of OFDM, $K=4$. 
The conventional and new SLM methods are different in two aspects:

- Method of generating random blocks,

- Selection rule.

In the suggested SLM method, each of $K$ different transforms of the generated block is a permuted version of the original sequence to avoid occurrence of consecutive high peaks. Therefore, the transmitter does not need the multiplication by pseudo-random sequences. To determine the selected permutation we have to transmit $\log _{2} K$ bits to the receiver as side information. In modified SLM, contrary to the original SLM, the transmitted block is not the one with the smallest PAPR. Figure 3-19 shows typical normalized power of OFDM and SM signals. In both signals, in addition to the largest peak, some other parts of the signal exceed the saturation level $\left(V_{s}\right)$. In OFDM, as opposed to SM, the largest peak of the signal is considerably larger than the other local peaks. Therefore a large portion of the distortion is generated due to the clipping of the largest peak.

In SM, where the local peak values are numerous and tend to have roughly similar magnitudes, they have equal effects on the distortion. Therefore the selection rule has to be modified from least PAPR to a rule which considers this property. It is appropriate to mention that other criteria, instead of least PAPR, have been recently proposed for the PTS method for OFDM [13]. Since all local peaks share the same effect on out-of-band radiation, we change the selection rule from least PAPR to least sum of squared errors between the amplifier input and output signals for an amplifier with unit gain. Therefore the metric which is the Sum of Squared Errors (SSE) would be: 


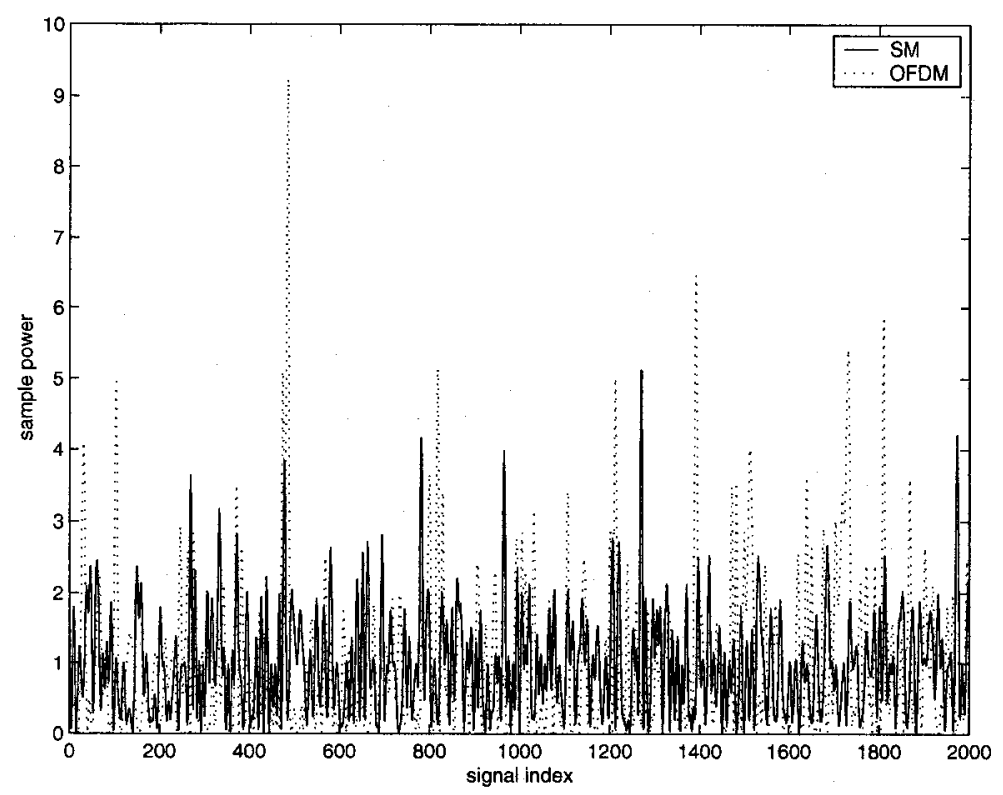

Figure 3-19. Typical OFDM and SM signal.

$$
S S E_{k}=\sum_{n=1}^{N M}\left|e_{k}(n)\right|^{2}=\sum_{n=1}^{N M}\left|v_{i, k}(n)-v_{o, k}(n)\right|^{2},
$$

where $k$ is the index of each permutation, $n$ is the sample index, $N$ is the number of samples per data block and $M$ is the over-sampling rate.

The distortion by HPA is partly generated by clipping the part of the signal above the saturation level and partly by HPA nonlinear characteristics up to the saturation level. PAPR reduction methods are usually useful for the distortion generated by clipping. As will be discussed in section 3.5.4, the effect of the nonlinearity up to the saturation level could be compensated by predistortion methods. Therefore in this section we only consider the Rapp model which is more linear than the Ghorbani model up to the saturation point especially for larger $p_{0}$. For the input signal well below the saturation level $\left(v_{i}<<V_{s}\right), v_{o} \cong v_{i}$ and the error signal is negligible. As $v_{i}$ approaches the saturation 
level, $v_{o}$ becomes less than $v_{i}$ and the error signal grows. To make the metric less complex, we just compute error when the input signal is above a determined threshold level $\left(v_{i, t h}\right)$. We define the threshold level $v_{i, t h}$ as an input signal value for which the ratio of the output to the input is a constant $C,\left(\frac{v_{o, t h}}{v_{i, t h}}=C\right.$ where $\left.C<1\right)$. Using (3-3) we can compute the threshold level in terms of $C$ :

$$
v_{i, t h}=V_{s} \sqrt[2 p_{0}]{(1 / C)^{2 p_{0}}-1}
$$

For example if we choose $C=97 \%$ or $C=99 \%$ for an amplifier with $p_{0}=5$ or $p_{0}=10$ respectively, the threshold would be $v_{i, t h}=0.9 V_{s}$. When the input signal is larger than the threshold, the output signal is between $0.9 V_{s}$ and $V_{s}$. Therefore instead of computing the exact corresponding output for each input, we can replace it by a fixed value between $0.9 V_{s}$ and $V_{s}$. The simulation results have shown that if we set the fixed value to $0.9 V_{s}$, it gives the best performance. Therefore the transmitter selects the permuted block which minimizes this metric:

$$
m_{k}=\sum_{n=1}^{N M}\left|e_{k}(n)\right|^{2}, 1 \leq k \leq K,
$$

where

$$
e_{k}(n)=\left\{\begin{array}{ll}
\left|v_{i, k}(n)\right|-0.9 V_{s} & \left|v_{i, k}(n)\right| \geq 0.9 V \\
0 & \text { otherwise }
\end{array},\right.
$$

In (3-13) $v_{i, k}(n)$ is the $n$-th sample of the signal in the $k$-th permutation, $V_{s}$ represents the nonlinear amplifier saturation level. The system requires transmitting $\log _{2} K$ bits as side 
information for each data block which is the same as the required side information for the SLM method in OFDM.

Simulation results show that this method considerably improves the envelope distribution and reduces the out-of-band radiation. The improvement achieved by the proposed SLM method for SM with $K=4$ is evident in Figure 3-20 in the form of the complementary cumulative distribution function. The probability of having a large peak is reduced by using the proposed SLM.

Out-of-band radiation of SM with modified SLM and OFDM with original SLM method for 16-QAM constellation are depicted in Figure 3-21, Figure 3-22 and Figure 3-23. In these figures we considered power back-off of $5 \mathrm{~dB}$ for an amplifier with $p_{0}=2$ and $p_{0}=10$ and back-off of $7 \mathrm{~dB}$ for $p_{0}=10$. SLM method can significantly decrease the out-of-band components, especially the first side lobe which causes interference for adjacent channels. These figures also show that for the same power back-off, the out-ofband radiation reduction achieved by the modified SLM algorithm in SM is larger than the reduction by original SLM in OFDM. The modified SLM method also reduces the required back-off in SM from 5.5 to $5 \mathrm{~dB}$ for the new reduced PSD. The corresponding value for the original SLM method in OFDM would be $5.3 \mathrm{~dB}$. These values change to 8 and $7.7 \mathrm{~dB}$ for SM and OFDM when the original back-off is $7 \mathrm{~dB}$. We note that for a given power back-off, SLM is more effective for an amplifier with large $p_{0}$, which is more linear up to the saturation level. In fact, SLM seems to be of little value for Rapp model with $p_{0}=2$ and also for Ghorbani model. This is due to the fact that all $K$ signals generated in the SLM method face the same amount of nonlinearity up to the saturation 




Figure 3-20. Modified SLM effect on CCDF of amplitude of Serial Modulation, $K=4$.

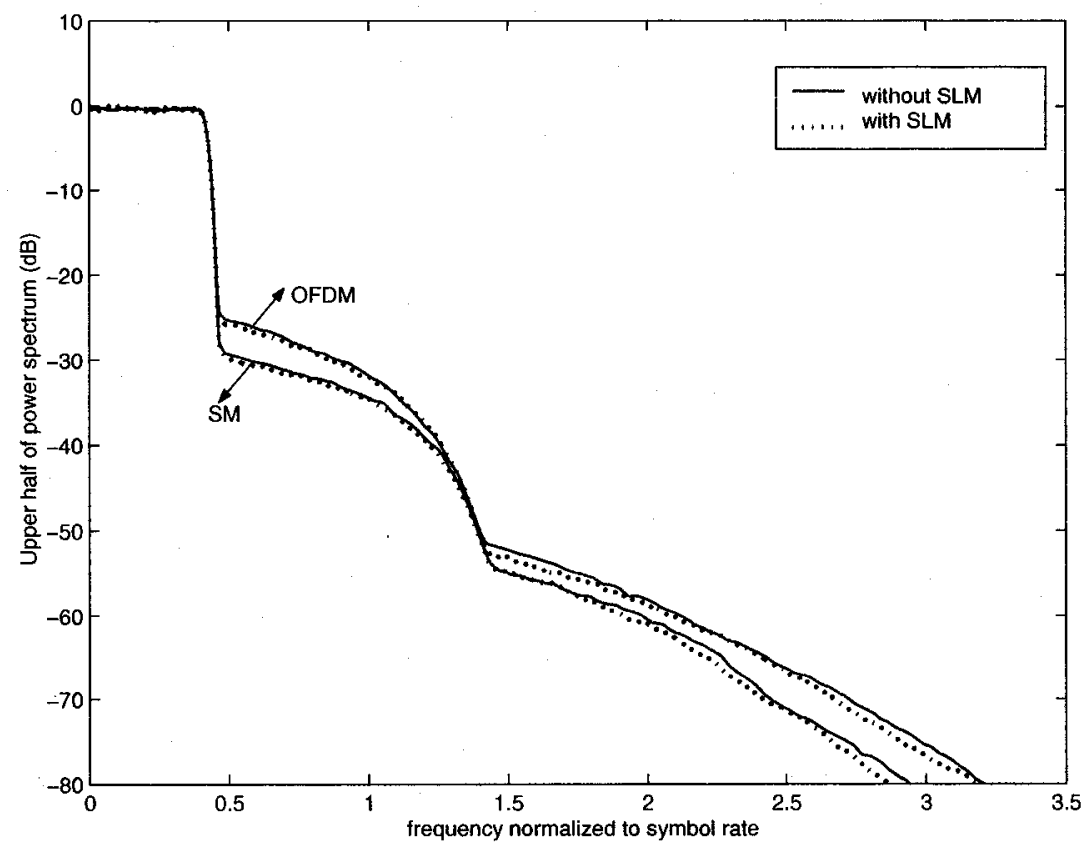

Figure 3-21. Reducing out-of-band radiation by SLM, $p_{0}=2, \mathrm{BO}=5 \mathrm{~dB}, K=4,16-\mathrm{QAM}$. 


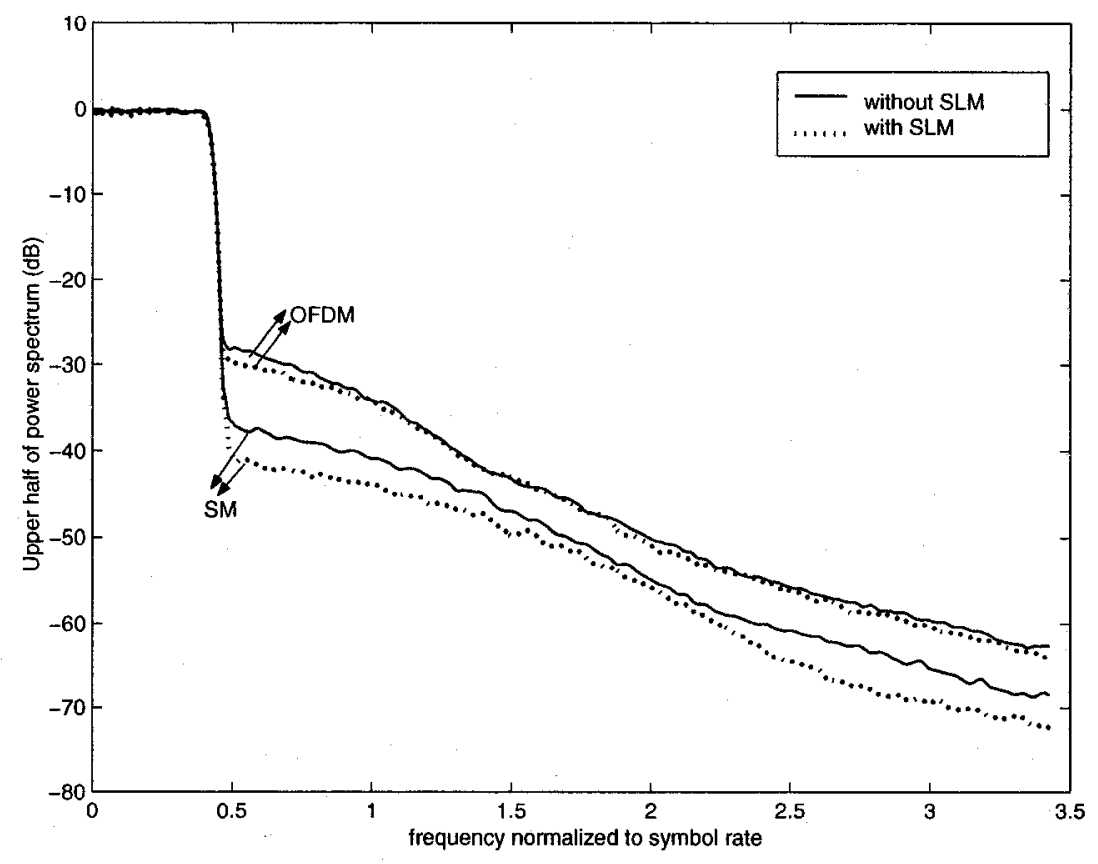

Figure 3-22. Reducing out-of-band radiation by SLM, $p_{0}=10, \mathrm{BO}=5 \mathrm{~dB}, K=4,16-\mathrm{QAM}$.

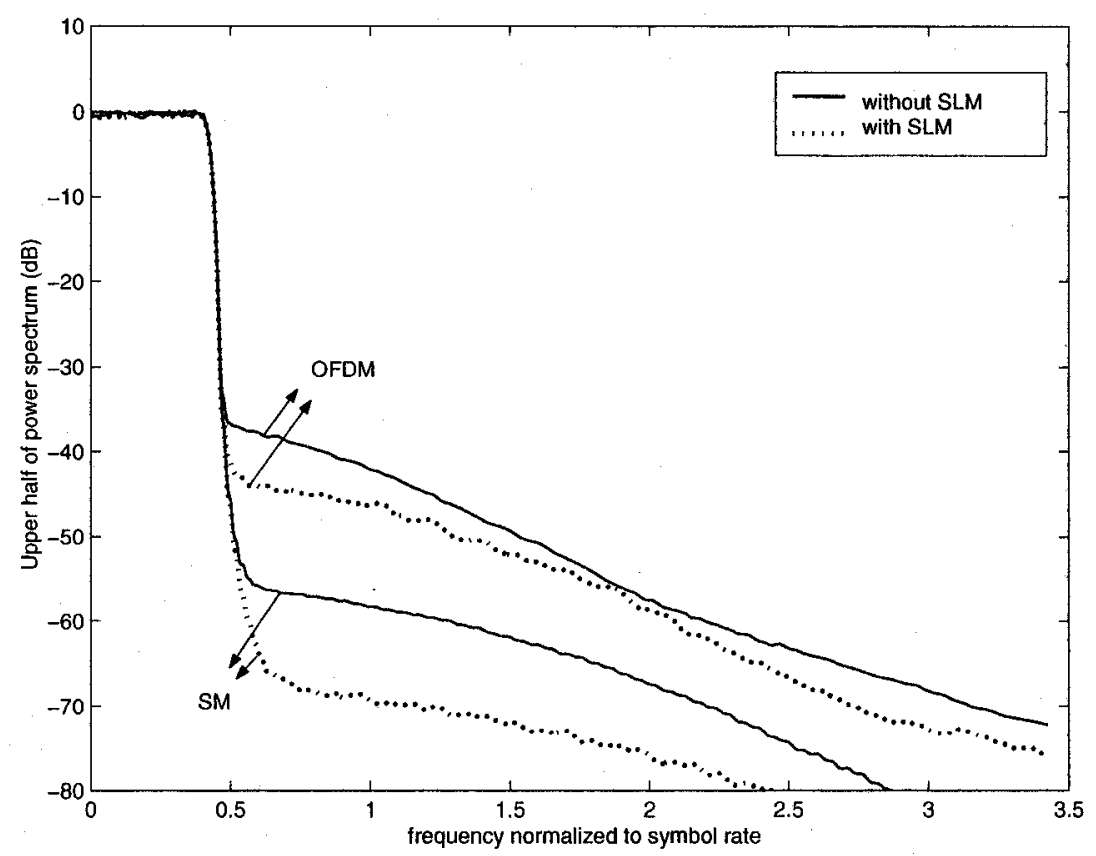

Figure 3-23. Reducing out-of-band radiation by $\mathrm{SLM}, p_{0}=10, \mathrm{BO}=7 \mathrm{~dB}, K=4,16-\mathrm{QAM}$. 
level. ${ }^{4}$ We use this fact to suggest a predistortion method in the next section to improve the performance of the proposed SLM method especially for smaller $p_{0}$ 's.

It is noteworthy that we also applied the new selection rule to the original SLM method for OFDM and found it not as efficient as the original selection rule. However by changing the multiplication by $\mathrm{PN}$ sequence to permutations, we were able to get results similar to those of original SLM method. It is good to mention that as confirmed by simulations the simplification of the SSE criterion to the form of (3-13) does not cause performance degradation.

\subsubsection{Improvements from Predistortion}

Predistortion is a well-known technique for the systems with nonlinear amplifiers [43]. The predistorter is placed prior to the amplifier and compensates for its nonlinearity. In this method, the input signal of the amplifier is deliberately predistorted to compensate for the nonlinear distortion caused by the amplifier. The ideal predistortion function is the inverse of $\mathrm{AM} / \mathrm{AM}$ or $\mathrm{AM} / \mathrm{PM}$ characteristics of the power amplifier so that the output signal would be linearly amplified up to the saturation level.

There are different categories of predistorters. Using a fixed look-up table as a predistorter is one of the simplest schemes. This method is efficient only if the AM/AM and AM/PM curves remain constant. If the amplifier characteristics change with time, adaptive algorithms should be performed to find the inverse curve of the amplifier characteristics. Recursive Least Square (RLS) and Least Mean Square (LMS) algorithms are among the most well-known methods for adaptive processing. RLS converges faster

${ }^{4}$ This fact is still valid if we change the threshold $\left(v_{i, t h}\right)$ or if we use the non-simplified SSE criterion in (3-12). 
than LMS although it is more complex. We can categorize predistorters to memoryless ones and those with memory. In this section we consider a look-up table as a memoryless predistorter which linearizes the AM/AM characteristic below the saturation level.

By using predistortion we prevent the performance degradation generated by nonlinear characteristics up to the saturation level. ${ }^{5}$ As discussed in section 3.3 (Figure 3-5 and Figure 3-6), the first side lobe of the PSD of an amplifier with smaller $p_{0}$ is more destructive than that of the one with larger $p_{0}$. On the other hand, for higher frequencies, out-of-band radiation would be lower for those with smaller $p_{0}$. However, usually for all amplifiers the out-of-band radiation at these frequencies is attenuated enough to be below the desired out-of-band radiation mask. By using a predistorter and making the amplifier linear up to the saturation point, we are able to reduce the first side lobe.

Another advantage of using predistorters is to improve the performance of the SLM algorithm. If we compare the performance of SLM for amplifiers with different $p_{0}$ 's, we will find that the larger the $p_{0}$ is, the more efficiently the SLM method reduces out-ofband radiation. When $p_{0}$ is small, a large portion of the out-of-band radiation is generated by the amplifier nonlinearity before the saturation level. SLM is of little value in this case. The reason is that any signal generated by SLM, even if its envelope is below the saturation level, is distorted by the nonlinearity before the saturation level. The only solution for this effect is to compensate for the nonlinearity of the amplifier. Based on this fact, we suggest using a predistortion technique in addition to the SLM method to make the amplifier perfectly linear up to the saturation level.

\footnotetext{
${ }^{5}$ Refer to Figure 3-11 and Figure 3-13.
} 
Figure 3-24 and Figure 3-25 show the PSD of a SM system when an ideal predistorter and SLM are employed separately or together for an amplifier with $p_{0}=5$ with power back-off of $5 \mathrm{~dB}$ and $7 \mathrm{~dB}$ respectively. Since the predistorter is ideal it completely compensates for the nonlinearity of the power amplifier and the overall effect of the amplifier and predistorter is linear up to the saturation level.

For back-off of $5 \mathrm{~dB}$, as opposed to the case for back-off of $7 \mathrm{~dB}$, the linearized HPA clips the signal selected by SLM. The considerable efficiency for back-off of $7 \mathrm{~dB}$ is due to the fact that there is no nonlinearity when the signal is below the saturation level because of the predistorter. Besides, the SLM algorithm performs well enough that the envelope of the selected signal never exceeds the saturation level. This makes the out-ofband radiation completely negligible.

\subsection{PAPR properties of SM-CDMA and MC-CDMA}

The combination of OFDM or SM with code division multiple access introduces two high rate techniques known as multi-carrier CDMA and single carrier direct sequence CDMA (which we will refer to as SM-CDMA). Both techniques benefit from the multipath- and interference-resistant properties of spread spectrum and the appealing frequency domain receiver simplicity of OFDM/SM systems [66][67]. As we only consider the up-link, the selection of the spreading codes whose combination generates the smallest PAPR in the base station is not in the scope of this chapter. Since there is an IDFT operation at the transmitter, a MC-CDMA signal, like OFDM, has large PAPR. The advantage of smaller PAPR in SM versus OFDM is implicitly extended to SMCDMA versus MC-CDMA in different papers [68][69][70][71]. In the rest of this chapter 


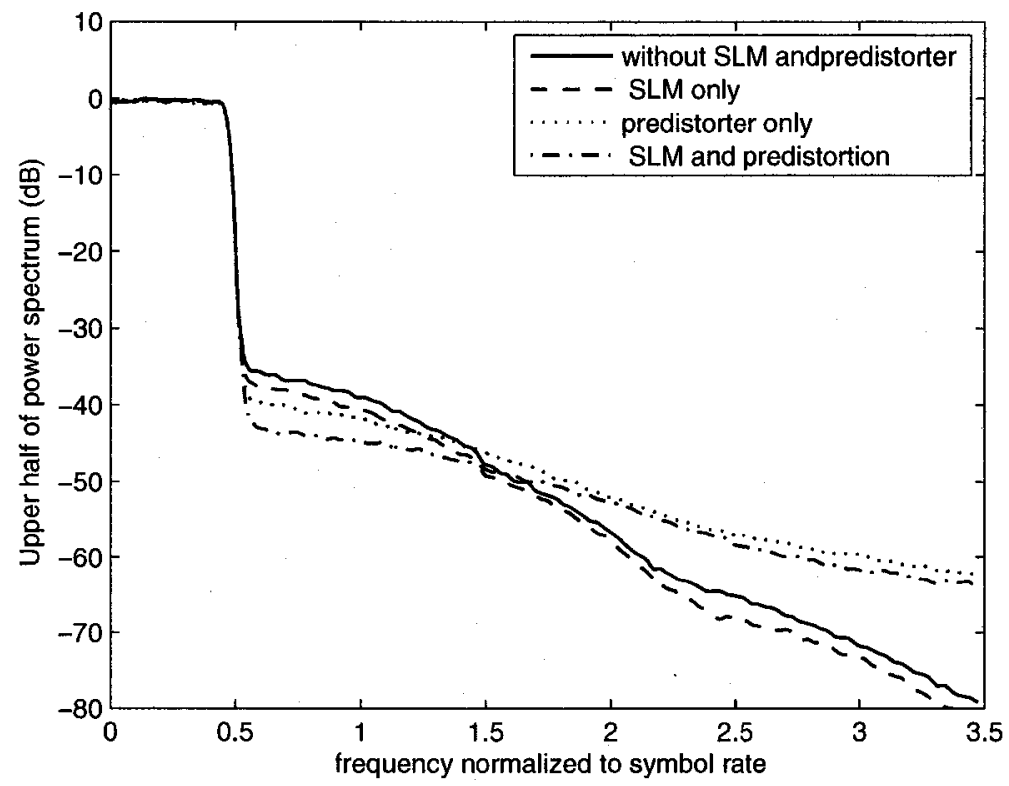

Figure 3-24. Effect of SLM and predistortion on reducing out-of-band radiation, $p_{0}=5, \mathbf{B O}=\mathbf{5 d B}$,

$K=4,16-\mathbf{Q A M}$.

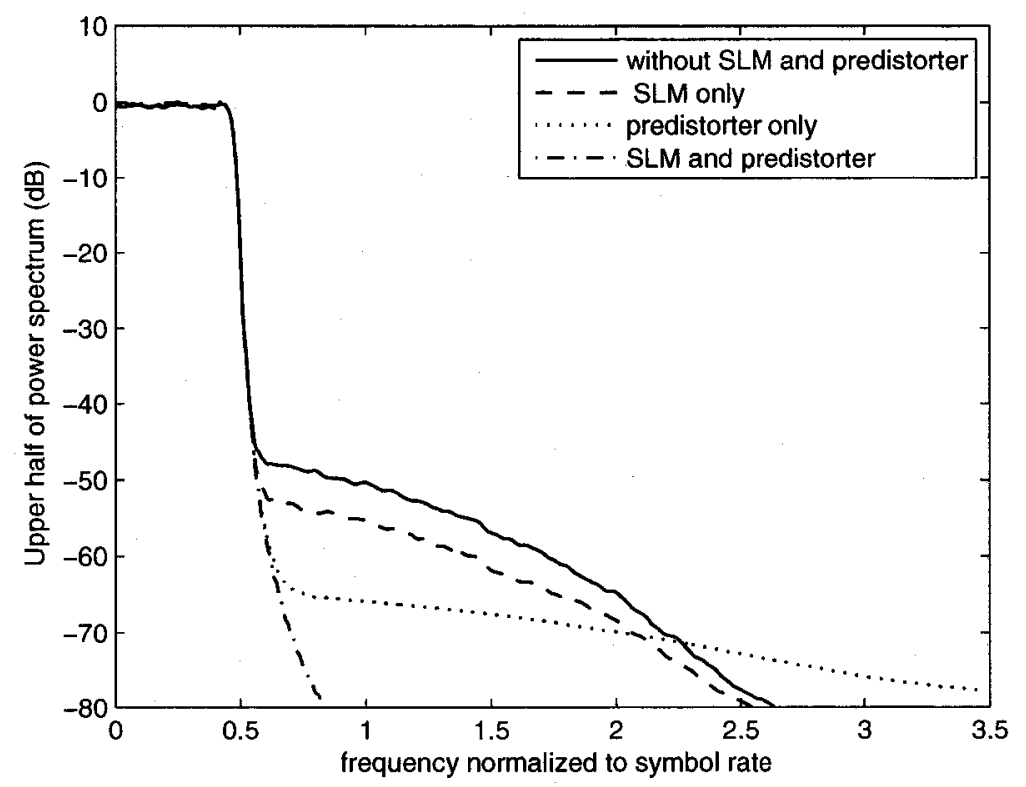

Figure 3-25. Effect of SLM and predistortion on reducing out-of-band radiation, $p_{0}=5, \mathrm{BO}=7 \mathrm{~dB}$, $K=4,16-Q A M$. 
we show that this extension is not always valid due to the PAPR increase in SM-CDMA (compared to that of SM) caused by the spreading. We show that this increase in the PAPR depends on both constellation type and spreading method. We consider equal-level constellations like QPSK and Offset QPSK (OQPSK) and multi-level constellations like 16-QAM as well as real and two complex spreading methods. To clarify the reason for the PAPR difference when various spreading methods are used, we derive the conditions in the original discrete data block which lead to a peak occurrence in the analog signal. This condition gives us a key to selecting the proper spreading method to generate smaller out-of-band radiation, thereby allowing use of an amplifier with smaller back-off. To the best of our knowledge there is no comprehensive investigation about the spreading effect on the PAPR of SM-CDMA and the comparison with that of MC-CDMA in the literature. Only in one section of [67] there is a simulation-based comparison between the PAPR of MC-CDMA and differentially encoded DS-CDMA.

\subsubsection{SM-CDMA}

We consider a system in which the equiprobable and i.i.d data bits are mapped to symbols $a_{n}$ and spread into chips $d_{n}$ by a spreading code $G_{k}$ with length $L$. Then the chips may be multiplied by a Pseudo-Noise (PN) sequence, $\Gamma_{i}$. Throughout this thesis, the function $f(x)=\lceil x\rceil$ rounds $x$ to the largest integer smaller than $x, \delta_{r}=\left\{\begin{array}{lll}1 & \text { if } & r=0 \\ 0 & \text { if } & r \neq 0\end{array}\right.$ and $u(r)=\left\{\begin{array}{lll}1 & \text { if } & r \geq 0 \\ 0 & \text { if } & r<0\end{array}\right.$. We consider real and complex spreading. In real spreading (RSP), after multiplication by the Walsh code, the signal is multiplied by a real-valued 
binary PN-sequence. In complex spreading I (C-SP I), real and imaginary parts are multiplied by different binary PN-sequences while in complex spreading II (C-SP II) the complex signal is multiplied by a complex PN sequence [72][73]. These spreading methods are presented in Figure 3-26 in which the multiplication of the symbol and Walsh code, $a G$, is presented by $I+j Q$. The chips of real and complex spreading I and II are calculated based on (3-14),(3-15) and (3-16) respectively.
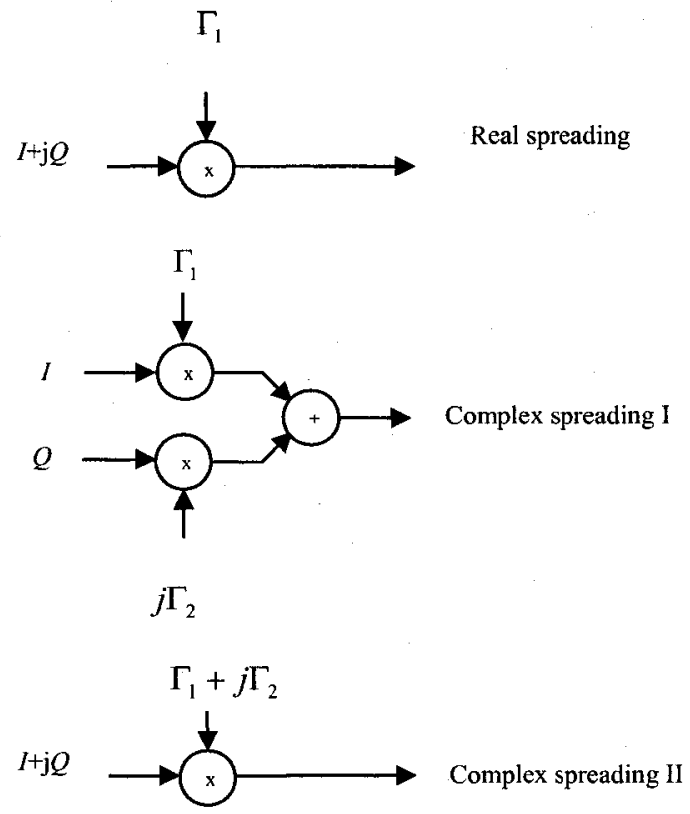

Figure 3-26. Different Spreading methods. 


$$
\begin{gathered}
d_{(m-1) L+k}=a_{m} G_{k} \Gamma_{1}((m-1) L+k), \\
d_{(m-1) L+k}=\operatorname{Re}\left(a_{m}\right) G_{k} \Gamma_{1}((m-1) L+k)+j \operatorname{Im}\left(a_{m}\right) G_{k} \Gamma_{2}((m-1) L+k), \\
d_{(m-1) L+k}=a_{m} G_{k}\left(\Gamma_{1}((m-1) L+k)+j \Gamma_{2}((m-1) L+k)\right) .
\end{gathered}
$$

The chips are transmitted in blocks including $N L$ chips where each block is preceded by a cyclic prefix with length $N_{C P}$. This length should be at least equal to the channel impulse response. By using a pulse shaping filter $(\tilde{h}()$.$) the discrete signal is converted to an$ analog signal as:

$$
s(t)=\sum_{n=-N_{C P}}^{N L-1} d_{n} \tilde{h}\left(t-n T_{c}\right),
$$

where $d_{n}$ and $T_{c}$ present the $n$-th chip and the chip duration respectively. For a large enough over-sampling rate, the analog signal can be approximated by the over-sampled signal as $s_{m}=\sum_{n=0}^{N L-1} d_{n} \tilde{h}_{m-n}$.

\subsubsection{PAPR of SM-CDMA}

For SM-CDMA, similar to SM, the PAPR is larger for multi-level constellations, and it would grow after filtering and D/A conversion, with high peak values after filtering typically being associated with the occurrence of several successive large-amplitude constellation points [16]. The peak of the signal after the D/A converter depends on both the amplitude and the phase of the consecutive waveform samples in the original block. First we consider a SM block and find the condition under which a peak occurs in the analog signal. We assume the original data block is $\underline{a}=\left[a_{0} \ldots a_{i} \ldots a_{N-1}\right]$. For simplicity we 
consider an equal-level constellation in which $a_{i}=\sqrt{E} e^{j \theta_{i}}$. If we over-sample ${ }^{6}$ the signal with rate $M$ to generate the vector $\mathbf{s}=\left[s_{0} \ldots s_{i} \ldots s_{N M-1}\right]$, we have:

$$
s_{m}=\left\{\begin{array}{l}
a_{n} \text { if } m=n M \\
\frac{1}{M N} \sum_{n=0}^{N-1} a_{n} \widetilde{h}_{m, n} \text { otherwise }
\end{array},\right.
$$

where $\widetilde{h}_{m, n}$ 's , the samples of the pulse shaping function, are:

$$
\widetilde{h}_{m, n}=e^{j \pi \frac{N-1}{N}\left(\frac{m}{M}-n\right)} \frac{\sin \left(\pi\left(n-\frac{m}{M}\right)\right)}{\sin \left(\frac{\pi}{N}\left(n-\frac{m}{M}\right)\right)}
$$

The phase of the pulse shaping filter samples (phase of $\widetilde{h}_{m, n}$ ) can be computed as:

$$
\varphi_{m, n}=\frac{m \pi}{M}-\frac{m \pi}{M N}+\left(\frac{(-1)^{\left[\frac{m}{M}\right]}-1}{2}\right) \pi+\Theta_{n} \text { where } \Theta_{n}=\left\{\begin{array}{lll}
\frac{n \pi}{N} & \text { if } & n<m / M \\
\frac{n \pi}{N}+\pi & \text { if } & n>m / M
\end{array} .\right.
$$

Therefore the magnitude of the $m$-th component of the over-sampled signal can be expressed as:

$$
\left|s_{m}\right|=\frac{\sqrt{E}}{M N}\left|\sum_{n<m / M}\right| \widetilde{h}_{m, n}\left|e^{j\left(\theta_{n}+\frac{m n}{N}\right)}+\sum_{n>m / M}\right| \widetilde{h}_{m, n}\left|e^{j\left(\theta_{n}+\frac{\pi n}{N}+\pi\right)}\right| .
$$

To maximize $\left|s_{m}\right|$, the components in both summations should have the same phase in order to add up constructively. Equivalently, the following equations should be satisfied:

$$
\theta_{n+1}=\left\{\begin{array}{lc}
\theta_{n}-\frac{\pi}{N} & n, n+1<m / M \text { or } n, n+1>m / M \\
\theta_{n}-\frac{\pi}{N}+\pi & n<m / M<n+1
\end{array}\right. \text {. }
$$

\footnotetext{
${ }^{6}$ For over-sampling we first take the FFT of the signal. We add $(M-1) N$ zeros at the end of the FFT block. Then we take the IFFT of the signal.
} 
For large $N, \frac{\pi}{N}$ is negligible and the conditions will be simplified to:

$$
\theta_{n+1}=\left\{\begin{array}{lc}
\theta_{n} & n, n+1<m / M \text { or } n, n+1>m / M \\
\theta_{n}+\pi & n<m / M<n+1
\end{array} .\right.
$$

In other words, the largest analog peak occurs when in the original data block there is a set of consecutive equal-phase symbols followed by another set of consecutive equalphase symbols. Additionally, the phase difference between the components of the two sets should be $\pi$. For instance the analog signal exhibits a large peak in the middle of the block if the original block consists of $N / 2$ symbols of ' $+a$ ' followed by $N / 2$ symbols of '$a^{\prime}$ as in $\underline{a}=[+a+a \ldots+a-a-a \ldots-a]$. The use of the cyclic prefix in block-systems, which produces the appearance of circular convolution, generates a fundamental difference between these systems and non-block systems. In block-systems the pulse shaping filter is shifted circularly and the tail of the function is folded back upon itself. The duration of the pulse shaping filter is also confined within the block duration. However in the non-block systems it decays over an infinite duration. Therefore the combination of symbols in the data sequence which results in a large peak in the analog signal, are different in these two systems. In non-block systems, the combination is as follows [72]:

$\ldots+a-a \ldots+a-a+a+a-a+a \ldots-a+a \ldots$

In this case, to have a large peak every two consecutive symbols should have the phase shift of $\pi$ except the two symbols in the middle (underlined in the sequence) which have equal phases. 
If (3-17) is not completely satisfied ${ }^{7}$, the signal exhibits a local peak which is less than the largest possible peak. This local peak can generate some out-of-band radiation. We should also note that (3-17) presents the condition for the SM signal.

In SM-CDMA, the original block is $\underline{d}=\left[d_{0} \ldots d_{i} \ldots d_{N L-1}\right]$. Therefore a condition similar to (3-17) between the chip phases should be satisfied. Since the consecutive chips which are related to one symbol are correlated, the probability of the occurrence of consecutive equal-phase chips in SM-CDMA is different from that of SM. This probability depends on the spreading method. In the following sub-sections we investigate the effect of different spreading methods on this probability for the constellations QPSK, OQPSK and 16-QAM.

\subsubsection{QPSK}

Based on (3-14), in real spreading the chips $\left(d_{(m-1) L+k}\right)$ related to one symbol $\left(a_{m}\right)$ will be equal either to $a_{m}$ or to $a_{m} e^{j \pi}$. Because of the different effect of PN sequences $\left(\Gamma_{1}\right.$ and $\Gamma_{2}$ ) in complex spreading I the possible constellation points for the chips are $a_{m} e^{j k \frac{\pi}{2}}$ for $k=0, . ., 3$. We consider $J$ consecutive symbols or chips as $b_{n+j}$ for $j=1, \ldots, J$ for SM and SM-CDMA respectively. If $P_{S M}, P_{\text {real }}$ and $P_{\text {complex }}$ show the probabilities of having the pattern of $b_{n+j}=\left\{\begin{array}{ll}+a & j=1, \ldots,\left\lceil\left\lceil\frac{m}{M}\right\rceil\right. \\ -a & j=\left\lceil\frac{m}{M}\right\rceil+1, \ldots, J\end{array}\right.$ which generates a local peak for SM, SM-CDMA

\footnotetext{
${ }^{7}$ For instance for a peak at the $m$-th sample of over-sampled signal, instead of having $\left\lceil\frac{m}{M}\right\rceil$ symbol of " $+a$ " followed by $N-\left\lceil\frac{m}{M}\right\rceil$ symbol of "- $a$ ", we have $m_{1}$ symbols of " $+a$ " followed by $J-m_{1}$ symbols of "-a" where $J<N$ and $m_{1}<\left\lceil\frac{m}{M}\right\rceil$ (even if $J<<N$ ).
} 
with real spreading and SM-CDMA with complex spreading respectively, these probabilities can be expressed as:

$$
\begin{gathered}
P_{S M}=P_{\text {complex }}=\frac{1}{4^{J}}, \\
P_{\text {real }}=\left(\frac{1}{4} \times \frac{1}{2^{L-1}}\right)^{\left\lceil\frac{J_{1}}{L}\right\rceil}\left(\frac{1}{4} \times \frac{1}{2^{r_{1}^{\prime}-1}}\right)^{\left(1-\delta_{\eta_{1}}\right)}\left(\frac{1}{4} \times \frac{1}{2^{L-r_{0}^{\prime}-1}}\right) u\left(J_{1}\right)+\left(\frac{1}{4} \times \frac{1}{2^{J-1}}\right)\left(1-u\left(J_{1}\right)\right),
\end{gathered}
$$

where $r_{0}^{\prime}$ and $r_{1}^{\prime}$ are remainders of the division $n$ and $J_{1}$ by $L$ respectively $\left(J_{1}=J-L-r_{0}^{\prime}\right)$. It is easy to see that $P_{S M}=P_{\text {complex }}<P_{\text {real }}$. For instance for the pattern “ $+a+a-a-a$ " when $J=4, L=8$ and $r_{0}=0$ we have $P_{S M}=P_{\text {complex }}=\frac{1}{256}$ while $P_{\text {real }}=\frac{1}{32}$. The difference between the probabilities increases for a larger $J$. We note that in complex spreading II, the chips are not equal-magnitude. However, since the difference between the chips amplitude is not considerable, as confirmed by simulations, both complex spreading methods for QPSK have similar PAPR.

\subsubsection{OQPSK}

In conventional non-block-wise single carrier systems, to reduce the PAPR we can employ OQPSK since there is no $\pi$ phase shift between the successive symbols. Prevention of simultaneous changes in the sign of inphase and quadrature components is achieved by delaying the quadrature component half of symbol duration compared to the inphase component. To use OQPSK in SM-CDMA, as depicted in (3-18) the delay between the inphase and quadrature components has to be equal to half of the chip period $\left(T_{c}\right):$ 


$$
s(t)=\sum_{n=-N_{C P}}^{N L-1} \operatorname{Re}\left(d_{n}\right) \tilde{h}\left(t-n T_{c}\right)+j \operatorname{Im}\left(d_{n}\right) \tilde{h}\left(t-n T_{c}-\frac{T_{c}}{2}\right) .
$$

As we prevent any $\pi$ phase shift between the real and imaginary parts of the signal in OQPSK, we expect that, unlike QPSK, real spreading performs well in terms of PAPR. Due to the $\frac{T_{c}}{2}$ delay in the quadrature component in OQPSK, the inphase component $\left(I_{n}\right)$ (after spreading by Walsh code and before multiplication by PN sequence $\Gamma_{i}$ ) overlaps in time with two quadrature components $\left(Q_{n-1}\right.$ and $\left.Q_{n}\right)$. Based on (3-15) and (3-16) we can demonstrate two consecutive discrete chips (after multiplication by PN sequences) in both complex spreading methods. These discrete chips are presented in (3-19) and (3-20) for complex spreading I and II respectively.

$$
\begin{gathered}
s(t)= \begin{cases}I_{n} \Gamma_{1}(n)+j Q_{n-1} \Gamma_{2}(n) & (n-1) T_{c}<t<\left(n-\frac{1}{2}\right) T_{c} \\
I_{n} \Gamma_{1}(n)+j Q_{n} \Gamma_{2}(n) & \left(n-\frac{1}{2}\right) T_{c}<t<n T_{c}\end{cases} \\
s(t)= \begin{cases}\left(I_{n}+j Q_{n-1}\right)\left(\Gamma_{1}(n)+j \Gamma_{2}(n)\right)=\left(I_{n} \Gamma_{1}(n)-Q_{n-1} \Gamma_{2}(n)\right)+j\left(I_{n} \Gamma_{2}(n)+Q_{n-1} \Gamma_{1}(n)\right) & (n-1) T_{c}<t<\left(n-\frac{1}{2}\right) T_{c} \\
\left(I_{n}+j Q_{n}\right)\left(\Gamma_{1}(n)+j \Gamma_{2}(n)\right)=\left(I_{n} \Gamma_{1}(n)-Q_{n} \Gamma_{2}(n)\right)+j\left(I_{n} \Gamma_{2}(n)+Q_{n} \Gamma_{1}(n)\right) & \left(n-\frac{1}{2}\right) T_{c}<t<n T_{c}\end{cases}
\end{gathered}
$$

As can be seen in (3-19) for complex spreading $I$, the inphase component $\left(I_{n} \Gamma_{1}(n)\right)$ is similar in both $\frac{T_{c}}{2}$ intervals. Therefore the mentioned inphase and quadrature parts can not change their signs simultaneously. However in complex spreading II, as can be 
confirmed by (3-20), $\pi$ phase shifts are possible between the inphase and quadrature components. Therefore we expect that by using complex spreading II the PAPR of SMCDMA with OQPSK will be larger than that of complex spreading I.

\subsubsection{16-QAM}

In (3-17) for the sake of simplicity we considered equal-level constellations. In multilevel constellations the high peaks depend not only on the phase of the original data but also on their amplitude. Usually the largest peak is associated with the occurrence of several successive large amplitude symbols. Since in real and complex spreading I we have $\left|d_{(m-1) L+k}\right|=\left|a_{m}\right|$ (for $k=0, \ldots, L-1$ ), in equal-level constellations all the chips have equal magnitudes. However in multi-level constellations the larger the original symbol amplitude is, the higher the chip amplitude will be. Assume $A_{\max }$ is the largest amplitude in the original constellation and $P_{m}$ is the probability of having a symbol with amplitude of $A_{\max }$ in the original constellation. Let $P_{S M-C D M A}$ and $P_{S M}$ be the probabilities of having two consecutive chips or symbols with the largest amplitude $\left(A_{\max }\right)$ of the original constellation before the D/A in SM-CDMA and SM, respectively. We have:

$$
P_{S M}^{\prime}=P\left(\left|a_{n}\right|=\left|a_{n+1}\right|=A_{\max }\right)=P_{m}^{2} \text {. }
$$

The corresponding probability for SM-CDMA is:

$$
\begin{aligned}
& P_{S M-C D M A}^{\prime}=P\left(\left|d_{n}\right|=\left|d_{n+1}\right|=A_{\max }\right) \\
& =P\left(\left|d_{n}\right|=\left|d_{n+1}\right|=A_{\max } \mid\left\lceil\frac{n}{L}\right\rceil=\left\lceil\frac{n}{L+1}\right\rceil\right) \frac{L-1}{L} \\
& +P\left(\left|d_{n}\right|=\left|d_{n+1}\right|=A_{\max } \mid\left\lceil\frac{n}{L}\right\rceil \neq\left\lceil\frac{n}{L+1}\right\rceil\right) \frac{1}{L}
\end{aligned}
$$




$$
\begin{aligned}
& =P\left(\left|a_{m}\right|=A_{\max }\right) \frac{L-1}{L}+P\left(\left|a_{m}\right|=\left|a_{m+1}\right|=A_{\max }\right) \frac{1}{L} \\
& =P_{m} \frac{L-1}{L}+P_{m}^{2} \frac{1}{L} .
\end{aligned}
$$

We can easily show that for $L>1$ and $P_{m}<1, P_{S M-C D M A}^{\prime}>P_{S M}^{\prime}$ (For equal-level constellations where $\left.P_{m}=1, P_{S M-C D M A}^{\prime}=P_{S M}^{\prime}\right)$. Therefore in multilevel constellations the probability of having a large peak in SM-CDMA is larger than in SM even for complex spreading. By a similar justification to the one in section 3.6.2.1, we can claim that the effect of real spreading in increasing the PAPR is more than for complex spreading.

\subsection{Simulation results}

To show the effect of different spreading methods on PAPR, we compare the out-ofband radiation and the amplitude CCDF for SM-CDMA, MC-CDMA and SM by simulation. The simulation parameters are as follows. Each data block contains 256 symbols selected from a QPSK, OQPSK or 16-QAM constellation. To have a fair comparison, we assume that the number of symbols in a SM block is equal to the number of chips in a SM-CDMA block. Signals are over-sampled with a factor of $M=8$. After adding a cyclic prefix, the data block is multiplied with a raised cosine time window with the roll-off factor of $\alpha_{t}=0.125$. The spreading factor is $L=8$. We use the Rapp model with $p_{0}=10$ and back-off of 5dB. Figure 3-27 and Figure 3-28 depict the CCDF of the amplitude of SM, MC-CDMA and SM-CDMA with real and complex spreading (I and II), when the constellation is QPSK/OQPSK and 16-QAM respectively. Figure 3-29 and Figure 3-30 show the corresponding out-of-band radiations. 


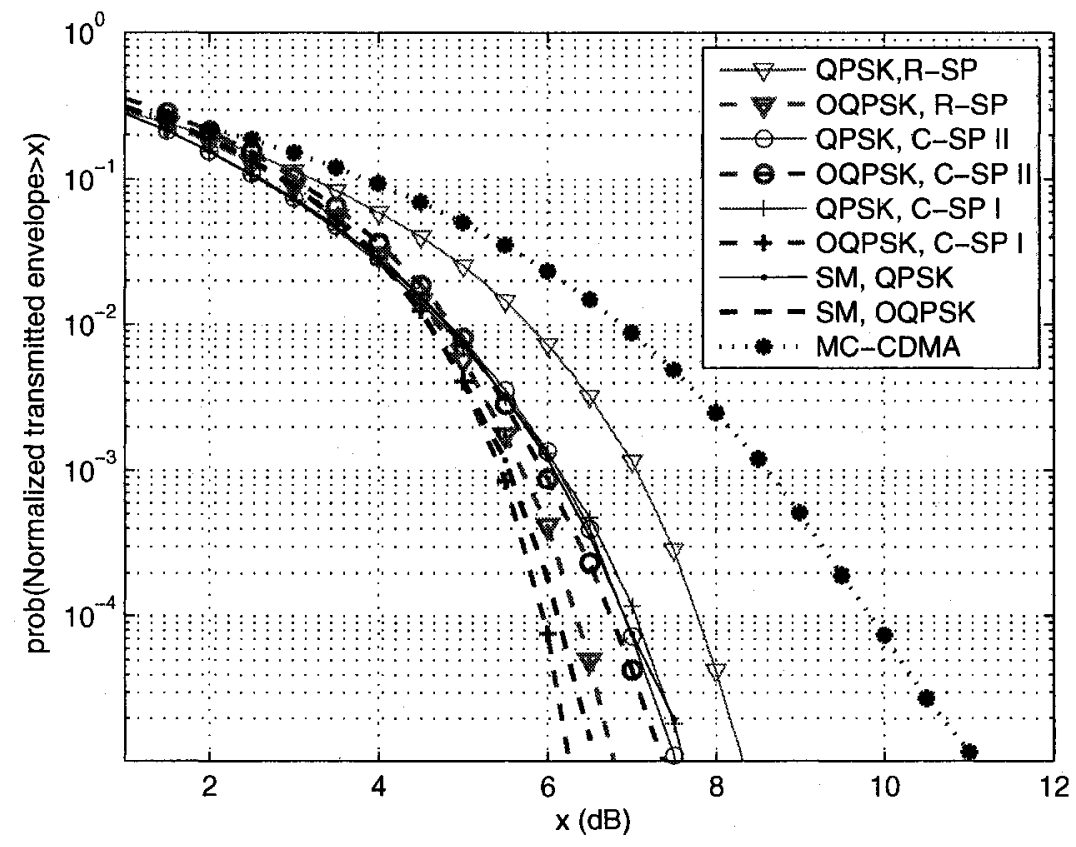

Figure 3-27. CCDF of the amplitude for SM, SM-CDMA and MC-CDMA, QPSK and OQPSK constellation.

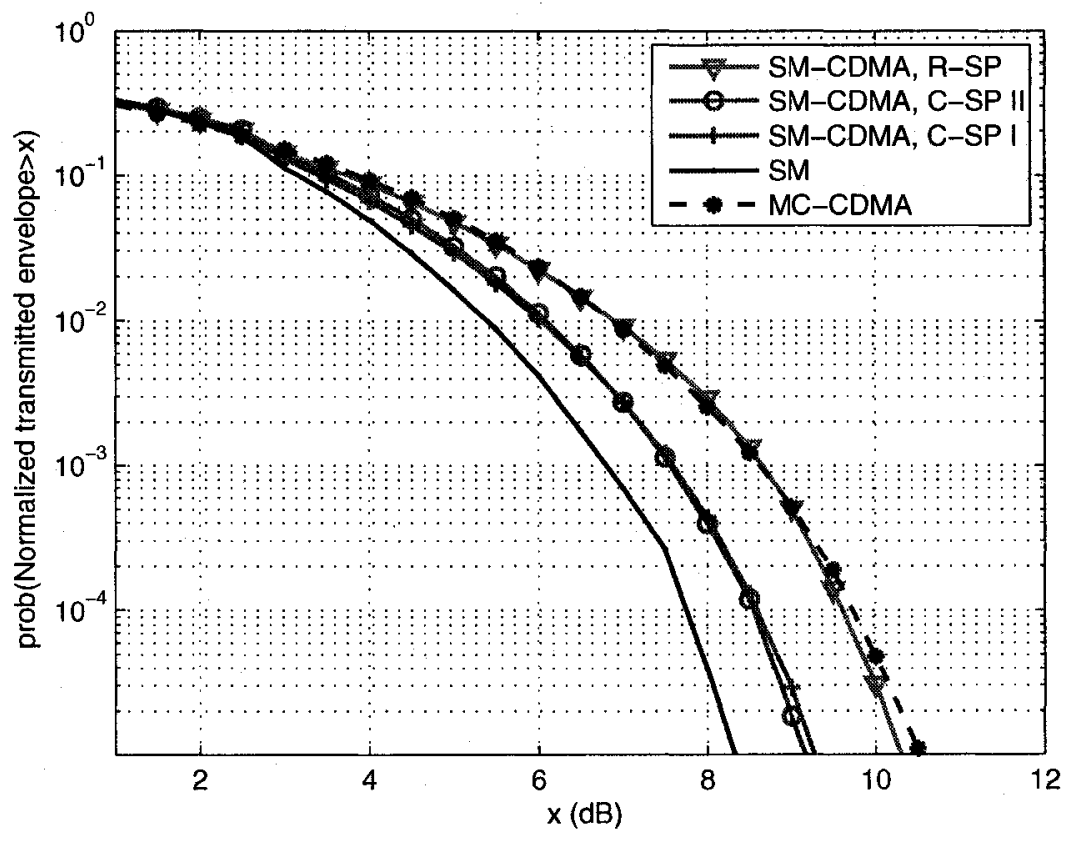

Figure 3-28. CCDF of the amplitude for SM, SM-CDMA and MC-CDMA, 16-QAM constellation. 


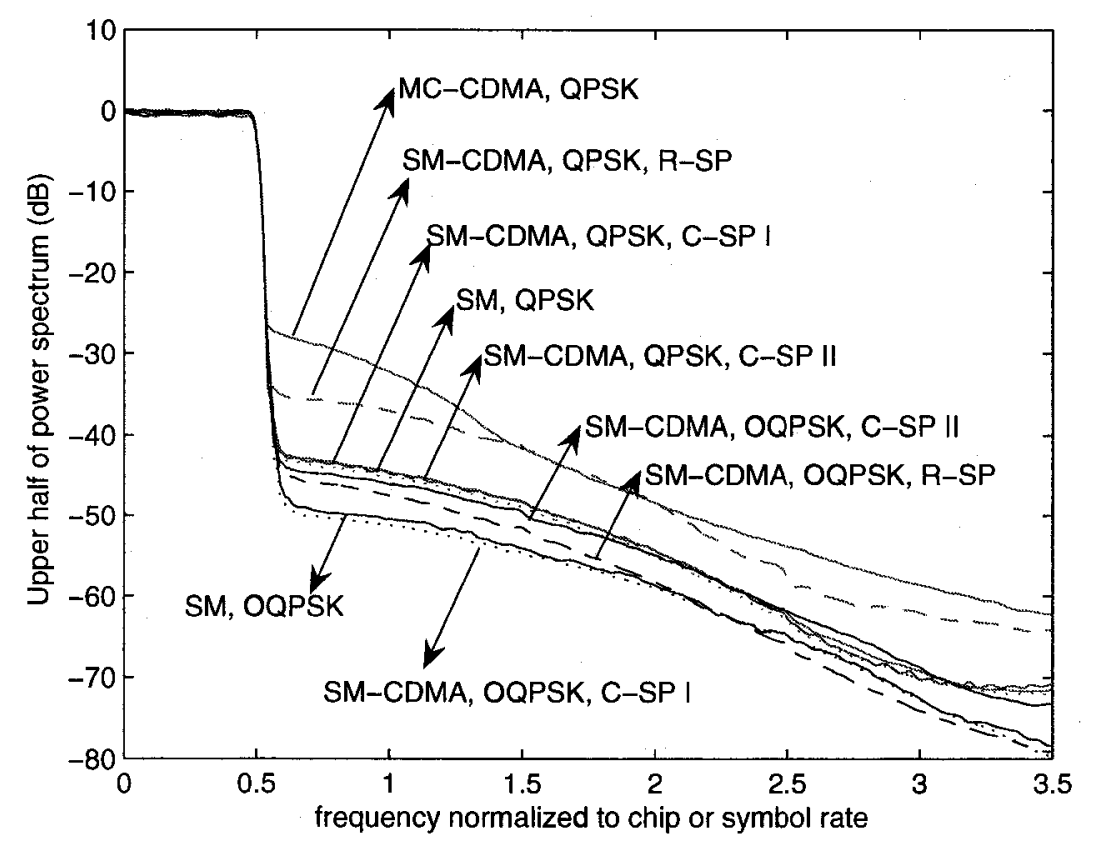

Figure 3-29. Out-of-band radiation of SM, SM-CDMA and MC-CDMA, QPSK and OQPSK constellation.

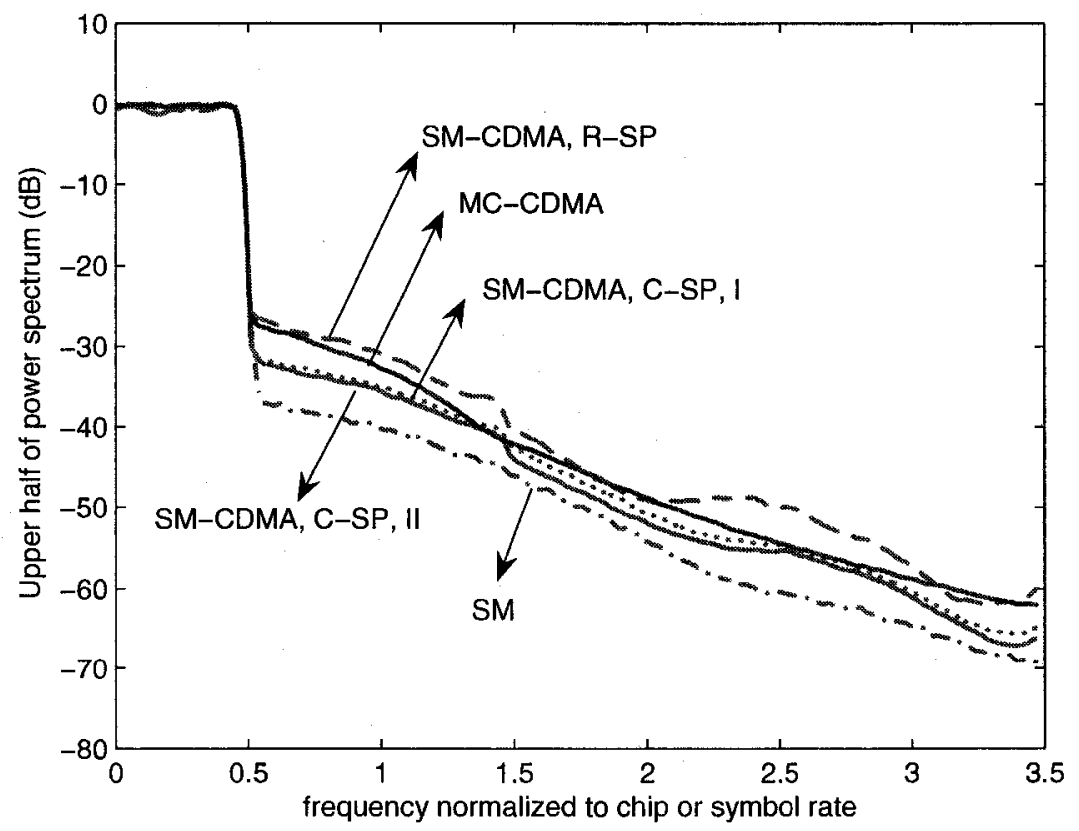

Figure 3-30. Out-of-band radiation of SM, SM-CDMA and MC-CDMA, 16-QAM constellation. 
As discussed in section 3.6.2.1, for QPSK we expect the out-of-band radiation of SMCDMA with complex spreading to be smaller than that of real spreading. It is observed that by using complex spreading the CCDF of SM-CDMA will become similar to that of SM.

It can be seen that the out-of-band radiation of OQPSK is smaller than that of QPSK for real and complex spreading I. Based on the discussion in section 3.6.2.2 we expect an increase in PAPR when complex spreading II is used in OQPSK which is confirmed by Figure 3-27 and Figure 3-29.

As explained in section 3.6.2.3, for 16-QAM for all spreading types the out-of-band radiation of SM-CDMA is larger than that of SM. For the same reason as QPSK the PAPR and out-of-band radiation of real spreading is larger than those of complex spreading. As presented in Figure 3-28 and Figure 3-30 the difference between complex spreading methods is almost negligible.

As discussed earlier, the advantage of SM versus OFDM is avoiding signals with high fluctuations. Taking into account that the PAPR of SM-CDMA can be larger than that of SM, we investigate if SM-CDMA has the same benefit compared to MC-CDMA. MCCDMA and OFDM have analogous amplitude distributions because the peaks are generated by adding signals constructively in the IFFT operation. By comparing the outof-band radiation in Figure 3-28 and Figure 3-30, we conclude that SM-CDMA is a more efficient method than MC-CDMA in terms of PAPR when complex spreading is used and especially for equal-level constellations like QPSK and OQPSK. However this efficiency no longer exists when the constellation is 16-QAM and real spreading is taken into service. 
A more practical approach to compare the mentioned cases is looking at the minimum HPA back-off value required to satisfy a specific spectral mask. Spectral masks determine the allowed amount of out-of-band radiation.

Table 3-1 presents the required back-off (found by simulations) to comply with a spectral mask to guarantee that the PSD does not exceed $-40 \mathrm{~dB}$. The results are completely consistent with our previous discussion about different spreading methods. The worst SM-CDMA case, which is $16-\mathrm{QAM}$ with real spreading, requires about $2.1 \mathrm{~dB}$ increase in the HPA back-off compared to that of SM. As can be seen the required back-off in this case is $7.5 \mathrm{~dB}$ which is very close to that of MC-CDMA. The best case is SM-CDMA with complex spreading I with OQPSK which needs an amplifier with back-off of $4.2 \mathrm{~dB}$.

It is worthwhile to mention that in SM and SM-CDMA we can reduce the required back-off (or guarantee achieving smaller out-of-band radiation) by using more efficient pulse shaping filters. For instance by using a raised cosine filter with roll-off factor of 0.1 or 0.2 , we can decrease the required back-off value by 0.5 and $1 \mathrm{~dB}$ respectively. However this is achieved at the expense of using some extra bandwidth.

As shown in section 3.6.2, out-of-band radiation of SM-CDMA may be larger than that of SM. The original SLM has been used to reduce PAPR of MC-CDMA [74][75].

Table 3-1 Minimum required HPA back-off value for out-of-band radiation less than $-40 \mathrm{~dB}$

\begin{tabular}{|c|c|c|c|c|c|}
\hline & SM & $\begin{array}{c}\text { SM-CDMA } \\
\text { Real spreading }\end{array}$ & $\begin{array}{c}\text { SM-CDMA } \\
\text { Complex spreading I }\end{array}$ & $\begin{array}{c}\text { SM-CDMA } \\
\text { Complex spreading II }\end{array}$ & MC-CDMA \\
\hline QPSK & $4.4 \mathrm{~dB}$ & $5.5 \mathrm{~dB}$ & $4.8 \mathrm{~dB}$ & $4.6 \mathrm{~dB}$ & $7.7 \mathrm{~dB}$ \\
\hline OQPSK & $4.2 \mathrm{~dB}$ & $4.4 \mathrm{~dB}$ & $4.2 \mathrm{~dB}$ & $4.6 \mathrm{~dB}$ & $\cdots$ \\
\hline $16-Q A M$ & $5.4 \mathrm{~dB}$ & $7.5 \mathrm{~dB}$ & $6.4 \mathrm{~dB}$ & $6.3 \mathrm{~dB}$ & $7.7 \mathrm{~dB}$ \\
\hline
\end{tabular}


Similarly we can use the modified SLM method, proposed in section 3.5.3, to reduce out-of-band radiation of SM-CDMA. This method can be used for SM-CDMA with the following metric:

$$
S S E_{k}=\sum_{n=1}^{N L M}\left|e_{k}(n)\right|^{2}=\sum_{n=1}^{N L M}\left|v_{i, k}(n)-v_{o, k}(n)\right|^{2},
$$

where $k$ is the index of each permutation, $n$ is the sample index and $L$ is the spreading gain. Figure 3-31 depicts the effect of using the modified SLM method to reduce out-ofband radiation of SM-CDMA when the constellation is 16-QAM and $p_{0}=10$ and four $(K=4)$ permuted blocks are generated in the transmitter for each original block. As depicted, the out-of-band radiation has been significantly reduced such that for 16-QAM and binary spreading which was the worst of the mentioned cases, out-of-band radiation has been made equal to that of SM. Equivalently we are able to use an amplifier whose back-off is $2 \mathrm{~dB}$ less than that of the original one and generates the same out-of-band radiation. Therefore even for the case that the amplitude distribution of SM-CDMA is worse than SM, we can improve its PAPR by using the modified SLM method. Thus the system can use amplifiers with smaller power back-off values.

\subsection{Conclusion}

In this chapter, we investigated the effect of nonlinear amplifier on the performance and out-of-band radiation of SM and OFDM as well as SM-CDMA and MC-CDMA. We also proposed a modified version of SLM algorithm to reduce the out-of-band radiation of SM and SM-CDMA. 


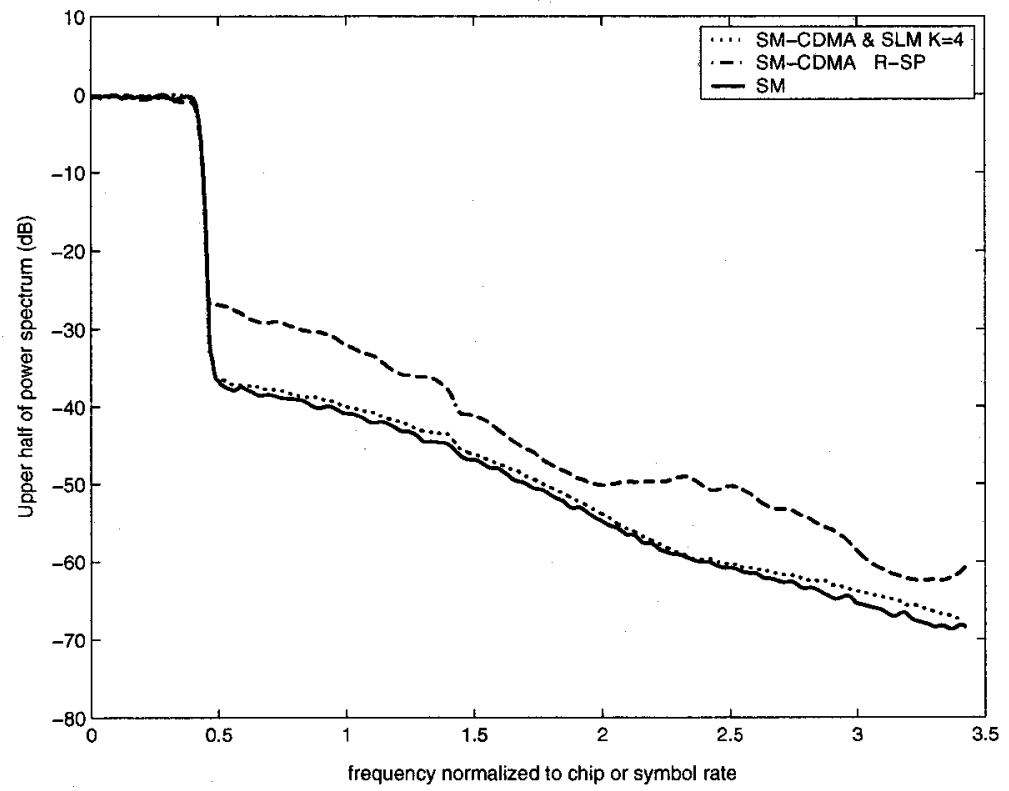

Figure 3-31. Out-of-band radiation reduction of SM-CDMA using SLM method. 


\section{Chapter 4 Turbo Frequency Domain}

\section{Equalization}

This chapter is partly background review (section 4.1 and section 4.3 .1 in which we describe turbo equalization and LDPC codes respectively). Section 4.2 is a prelude to section 4.3 and Chapter 5. In section 4.3.2 and section 4.3.3 we compare the performance and complexity of adaptive and non-adaptive TFDE with different coding methods respectively. 


\subsection{Turbo Equalization}

To combat the effect of inter symbol interference, different equalization methods have been proposed; among them are linear, decision feedback and maximum likelihood Equalization. Turbo Equalization, suggested in 1995, is a method in which the equalization and decoding are performed jointly, soft information being exchanged between the equalizer and the decoder iteratively [45]. The fundamental difference between this method and the conventional equalization methods is the use of soft information provided by the decoder to achieve better performance in the equalization. The system can achieve good performance by using an efficient but complex Maximum A Posteriori (MAP) equalizer. By replacing the MAP equalizer with a minimum mean squared error (MMSE) equalizer in a soft interference cancellation (SIC) scheme the system is less complex yet achieves good performance [46][55]. Implementing the MMSE equalizer in the frequency domain has been proposed to make the system even less complex [47][48].

Figure 4-1 depicts the block diagram of a turbo equalizer. The soft information generated by each module is used as a priori information by the other one. As iterations continue, if the reliability of the decoder decisions increases, the equalizer continually improves the reliability of its output.

\subsection{SIC-Turbo Frequency Domain Equalization}

We consider a system in which the equiprobable and i.i.d data bits are first encoded by an error correcting code. Each $K_{1}$ coded bits $\left(c_{n, k}, k=1, . ., K_{1}\right)$ are mapped to symbols $\left(x_{n}\right)$ with average power $\sigma_{d}^{2}$ selected from the constellation $S$ including $2^{K_{1}}$ points. For 
BPSK $S=\{+1,-1\}$, for 4-QAM $S=\{ \pm 1 \pm j\}$ and for 16-QAM, $S=\left\{s_{r}+j s_{i}\right\}$ where $s_{r}, s_{i} \in\{ \pm 1, \pm 3\}$ and bits are mapped to symbols by Gray mapping. The modulated symbols are transmitted in a block with length $N$ which is preceded by a cyclic prefix whose length at least equals the channel impulse response.

The sampled channel impulse response is $h[n]=\sum_{k=0}^{N_{h}-1} h_{k} \delta[n-k]$ and the channel noise is white Gaussian with variance of $\sigma^{2} . H_{k}$ is the $k$-th component of the channel frequency response which is the DFT of the sampled impulse response. $\mathrm{C}_{h}$ denotes the $N \times N$ circulant channel matrix ${ }^{8} \mathbf{C}_{h}=\operatorname{Circ}_{N}\left[h_{0} \ldots h_{N_{h}-1}\right]$. In what follows, we define function $Q($.$) as Q(x) \stackrel{\Delta}{=} \frac{1}{\sqrt{2 \pi}} \int_{x}^{+\infty} e^{\frac{-t^{2}}{2}} d t$.

\subsubsection{Non-adaptive TFDE}

Turbo equalization, like any other turbo processor includes two soft input soft output (SISO) processors exchanging soft information. Since the optimum solution of MAP equalization is too complex, we use the suboptimal but effective MMSE equalization in a soft interference cancellation scheme [55]. Due to the simplicity of the equalization in the frequency domain compared to the time domain, we implement the MMSE filter in the frequency domain. This requires block transmission which allows us to use FFT and IFFT to convert the signals from time to frequency domain and vice versa. As presented in the turbo frequency domain equalizer block diagram in Figure 4-2, in the feedback loop a soft estimation of the interference signal is generated and subtracted from the

\footnotetext{
${ }^{8}$ Refer to Appendix B for more details.
} 


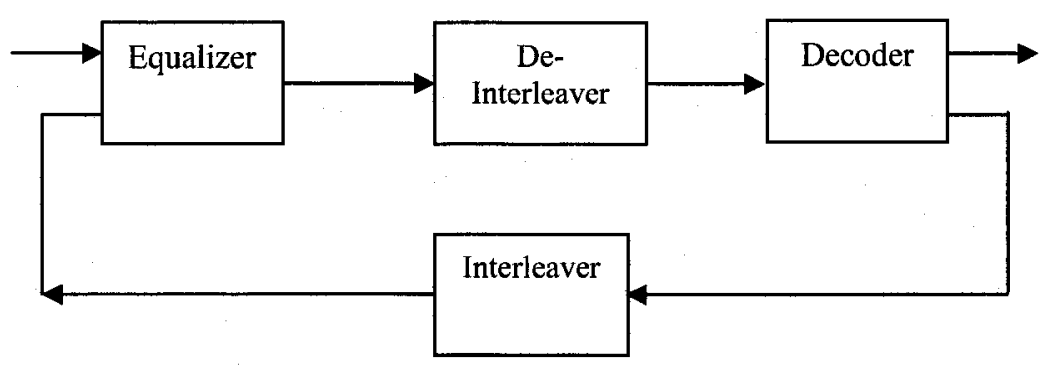

Figure 4-1. Block diagram of a turbo equalizer.

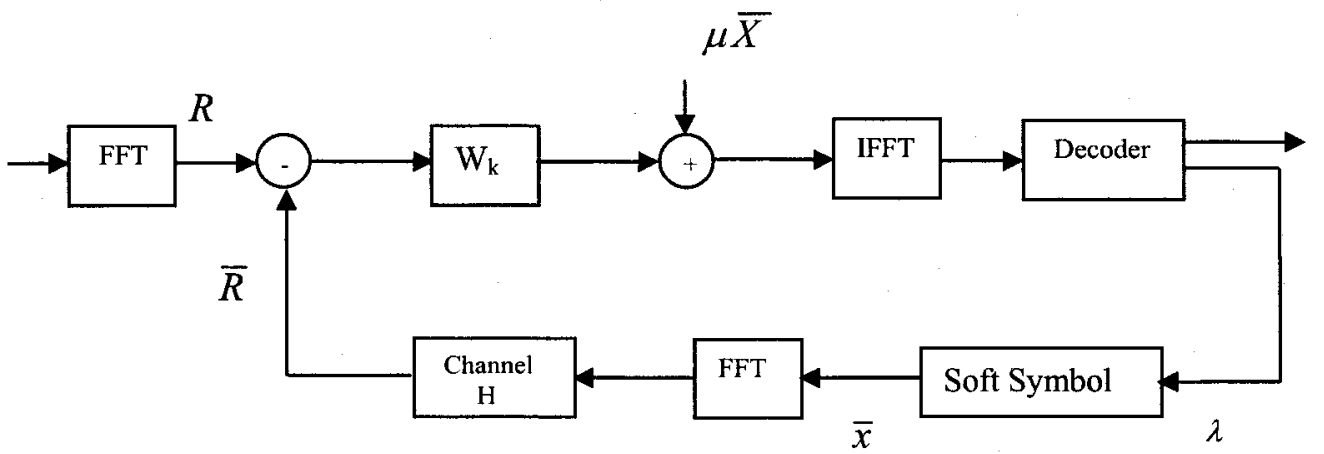

Figure 4-2. Block Diagram of SIC Turbo Frequency Domain Equalizer. 
received signal. The remaining is a residual ISI which is removed by the MMSE frequency domain equalizer. The vector of equalizer $\left(\mathbf{w}_{n}\right)$ coefficients in the time domain are as follows [55]:

$$
\mathbf{w}_{n}=\left(\left(\sigma^{2} \mathbf{I}_{N \times N}+\mathbf{C}_{h} \mathbf{v}_{n} \mathbf{C}_{h}^{H}+\left(\sigma_{d}^{2}-v_{n}\right) \widetilde{\mathbf{s}} \widetilde{\mathbf{s}}^{H}\right)^{-1} \widetilde{\mathbf{s}} \sigma_{d}^{2}\right)^{*}
$$

where $\widetilde{\mathbf{s}}=\mathbf{C}_{h}\left[\begin{array}{ll}1 & \mathbf{0}_{1 \times N-1}\end{array}\right]^{T}$ and $\mathbf{0}_{M \times N}$ is an $M \times N$ matrix with zero elements and $(.)^{\mathrm{T}}$ is the transpose operator. The details of (4-1) can be found in [55]. In (4-1), $\mathbf{v}_{n}$ is a $N \times N$ diagonal matrix whose $n$-th diagonal element, $v_{n}$ is defined as $\operatorname{Cov}\left(x_{n}, x_{n}\right)=E\left(x_{n} x_{n}^{*}\right)-\left|E\left(x_{n}\right)\right|^{2}$. To obtain $v_{n}, E\left(x_{n}\right)$ (which is represented by $\left.\bar{x}_{n}\right)$ and $E\left(x_{n} x_{n}^{*}\right)$ should be determined. Without a priori information, symbols $\left(x_{n}\right)$ are equiprobable. Hence $\bar{x}_{n}=0$ and $E\left(x_{n} x_{n}^{*}\right)=\sigma_{d}^{2}$. In the presence of a priori information, $\bar{x}_{n}$ which is the soft estimation of the $n$-th symbol is calculated as:

$$
\bar{x}_{n}=E\left(x_{n} \mid z_{n}\right)=\sum_{s \in S} P\left(x_{n}=s \mid z_{n}\right) \times s .
$$

The value of $P\left(x_{n}=s \mid z_{n}\right)$ can be determined based on each encoded bit LLR generated by the decoder. We denote the LLR of the $k$-th bit of the $n$-th symbol by $\lambda_{n, k}$ which is defined as:

$$
\lambda_{n, k}=\ln \frac{P\left(c_{n, k}=0 \mid z_{n}\right)}{P\left(c_{n, k}=1 \mid z_{n}\right)}
$$

The magnitude of $\lambda_{n, k}$ determines the reliability of the information. The larger the difference between $P\left(c_{n, k}=0 \mid z_{n}\right)$ and $P\left(c_{n, k}=1 \mid z_{n}\right)$ is, the larger the value of LLR will 
be. Ultimately, when $P\left(c_{n, k}=0 \mid z_{n}\right)=1$ and $P\left(c_{n, k}=1 \mid z_{n}\right)=0, \lambda_{n, k}$ approaches plus infinity. On the other hand, when the probabilities are equal, LLR would be zero indicating that there is no information about that specific bit.

Based on (4-3) the a priori probabilities can be expressed as:

$$
\begin{gathered}
P_{n, k} \stackrel{\Delta}{=} P\left(c_{n, k}=0 \mid z_{n}\right)=\frac{e^{\lambda_{n, k}}}{1+e^{\lambda_{n, k}}} . \\
1-P_{n, k}=P\left(c_{n, k}=1 \mid z_{n}\right)=\frac{1}{1+e^{\lambda_{n, k}}} .
\end{gathered}
$$

By replacing (4-4) and (4-5) in (4-2) we can find the soft estimation of the $n$-th symbol for different constellations. For BPSK it would be:

$$
\bar{x}_{n}=P_{n, 1}-\left(1-P_{n, 1}\right)=\tanh \left(\frac{\lambda_{n, 1}}{2}\right)
$$

When the signal is selected from a 4-QAM constellation, we have:

$$
\bar{x}_{n}=P_{n, 1}-\left(1-P_{n, 1}\right)+j\left[P_{n, 2}-\left(1-P_{n, 2}\right)\right]=\tanh \left(\frac{\lambda_{n, 1}}{2}\right)+j \tanh \left(\frac{\lambda_{n, 2}}{2}\right) .
$$

Since the system for 4-QAM can be seen as two dimensional BPSK, in the rest of this chapter and the next chapter we only consider BPSK and 16-QAM. For 16-QAM, considering Gray mapping presented in Figure 4-3, the conditional probability of each symbol, $P\left(x_{n}=s \mid z_{n}\right)$, can be expressed based on bits' probabilities as:

$$
P\left(x_{n}=s \mid z_{n}\right)=\prod_{k=1}^{4}\left[\left(1-b_{k}\right) P_{n, k}+b_{k}\left(1-P_{n, k}\right)\right]
$$

where $b_{k}$ is the $k$-th bit associated with the symbol $s$. For instance:

$$
P\left(x_{n}=-3+3 j \mid z_{n}\right)=P_{n, 1} P_{n, 2} P_{n, 3} P_{n, 4},
$$




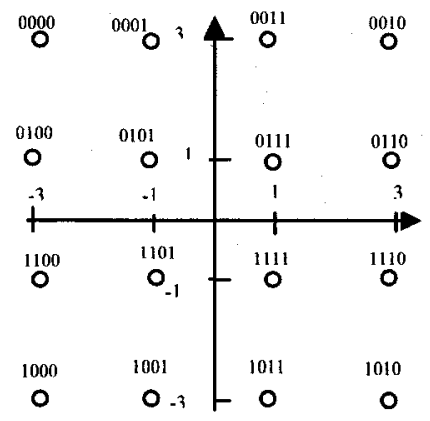

Figure 4-3. Gray mapping for 16-QAM.

$$
P\left(x_{n}=-1+3 j \mid z_{n}\right)=P_{n, 1} P_{n, 2} P_{n, 3}\left(1-P_{n, 4}\right) .
$$

By replacing these probabilities in (4-2), the real $(\operatorname{Re}()$.$) and imaginary (\operatorname{Im}()$.$) parts of$ soft estimated symbol are found as:

$$
\begin{aligned}
& \operatorname{Re}\left(\bar{x}_{n}\right)=\left(1-2 P_{n, 3}\right)\left(1+2 P_{n, 4}\right), \\
& \operatorname{Im}\left(\bar{x}_{n}\right)=\left(2 P_{n, 1}-1\right)\left(1+2 P_{n, 2}\right) .
\end{aligned}
$$

Equivalently we can rewrite the soft symbol of 16-QAM based on the bit LLR as:

$$
\bar{x}_{n}=-\tanh \left(\frac{\lambda_{n, 3}}{2}\right)\left(2+\tanh \left(\frac{\lambda_{n, 4}}{2}\right)\right)+j \tanh \left(\frac{\lambda_{n, 1}}{2}\right)\left(2+\tanh \left(\frac{\lambda_{n, 2}}{2}\right)\right)
$$

To find $v_{n}$ we should also evaluate $E\left(x_{n} x_{n}^{*}\right)$. In equal level constellations like BPSK or 4-QAM, even in the presence of a priori information, $E\left(x_{n} x_{n}^{*}\right)$ is constant and equal to $\sigma_{d}^{2}$. For 16-QAM, it depends on the bits' LLR and mapping. For the mapping illustrated in Figure 4-3 it can be written as: 


$$
\begin{aligned}
E\left(x_{n} x_{n}^{*}\right) & =\sum_{s \in S}|s|^{2} P\left(x_{n}=s \mid z_{n}\right) \\
& =8 P_{n, 2}+8 P_{n, 4}+2 \\
& =4 \tanh \left(\frac{\lambda_{n, 2}}{2}\right)+4 \tanh \left(-\frac{\lambda_{n, 4}}{2}\right)+10
\end{aligned}
$$

To find the equalizer parameters in the frequency domain, we multiply (4-1) by the FFT matrix (F) with elements of $F_{i, k}=\exp \left(-j \frac{2 \pi(i-1)(k-1)}{N}\right)$ for $k, i=1, \ldots, N$. The design of the frequency domain equalizer can be with or without considering the feedback information. Accordingly the equalizer would be either adaptive or non-adaptive during the iterations.

If the MMSE estimation is performed without considering the a priori information, as presented in Appendix B the equalizer coefficients which are fixed during the iterations are as follows [48]:

$$
W_{k}=\frac{H_{k}^{*}}{\frac{\sigma^{2}}{\sigma_{d}^{2}}+\left|H_{k}\right|^{2}}, k=1, \ldots, N
$$

\subsubsection{Adaptive TFDE}

To improve the performance of the equalizer structure in (4-10), we can use the a priori information. As discussed in Appendix C, if we replace $\mathbf{v}_{n}$ by $\mathbf{v}_{n}=v \mathbf{I}_{N \times N}$ where 
$\nu=\frac{1}{N} \sum_{n=1}^{N} v_{n}{ }^{9}$, define $\mathbf{A} \stackrel{\Delta}{=} \sigma^{2} \mathbf{I}_{N \times N}+v \mathbf{C}_{h} \mathbf{C}_{h}^{H}$ and apply the matrix inversion lemma (MIL) to (4-1), we will have:

$$
\mathbf{w}_{n}=\left(\mathrm{KA}^{-1} \widetilde{\mathbf{s}}\right)^{*}
$$

where $_{\mathrm{K}}=\frac{\sigma_{d}^{2}}{1+\left(\sigma_{d}^{2}-v\right) \widetilde{\mathbf{s}}^{H} \mathbf{A}^{-1} \widetilde{\mathbf{s}}}$. By converting the formula to the frequency domain, the filter coefficients would be:

$$
W_{k}=\mathrm{K} \frac{H_{k}^{*}}{\sigma^{2}+v\left|H_{k}\right|^{2}}, k=1, \ldots, N
$$

where $\mathrm{K}$ is a constant for all $k=1, \ldots, N$. For both adaptive and non-adaptive structures, the estimated symbol in the frequency domain at the output of the equalizer would be:

$$
Z_{k}=W_{k}\left(R_{k}-\bar{R}_{k}\right)+\mu \bar{X}_{k}=W_{k} R_{k}-W_{k} H_{k} \bar{X}_{k}+\mu \bar{X}_{k},
$$

where $\overline{\mathbf{X}}=\left[\bar{X}_{1} \ldots \bar{X}_{N}\right]=F F T(\overline{\mathbf{x}})=F F T\left(\left[\bar{x}_{1} \ldots \bar{x}_{N}\right]\right)$ and

$$
\mu=\frac{1}{N} \sum_{k=1}^{N} H_{k} W_{k}
$$

In the following section we compare the complexity and performance of adaptive and non-adaptive TFDE. The coding method we used in TFDE in the rest of this thesis is LDPC codes. Since convolutional codes are usually used in turbo equalizations, we compare the performance and complexity of TFDE with these coding methods for BPSK to justify using LDPC codes in TE.

\footnotetext{
9 As mentioned in Appendix $\mathrm{C}$, if we change the criterion from minimizing mean squared error to minimizing average mean squared error, without using this approximation we achieve a similar equation for the equalizer [76].
} 


\subsection{Comparison of TFDE using different coding methods}

Traditionally the coding method used in turbo equalization is convolutional coding. Recently other methods such as LDPC codes have been suggested for the encoder/decoder parts [50][51][52]. However in the literature, there is no complexity/performance comparison between the two mentioned turbo systems. Furthermore, in all papers using LDPC codes, the equalization was in the time domain while we consider SIC-TFDE. In section 4.3.1 we briefly review LDPC codes, in sections 4.3.2 and 4.3.3 we compare the performance and complexity of the two mentioned systems.

\subsubsection{Introduction to LDPC codes}

LDPC is a powerful coding method especially when the code length is sufficiently large [78]. It is a linear block code specified by its sparse parity check matrix $\widetilde{\mathbf{H}}$. Code words should satisfy parity check equations determined by $\underline{c} \widetilde{\mathbf{H}}^{T}=0$ in which $\underline{c}$ is a code word and $(.)^{T}$ presents the transpose. The parity check matrix of a regular $\left(i_{c} j_{c}\right)$ LDPC code has exactly $i_{c}$ and $j_{c}$ ones in each column and each row respectively. Thus each bit is involved in $i_{c}$ parity check constraints and each parity check equation includes $j_{c}$ bits. In irregular

codes, all columns and rows of $\widetilde{\mathbf{H}}$ do not contain the same number of ones necessarily. A very helpful method for representing the parity check matrix and the relation between bits and parity check equations is the Tanner graph. The Tanner graph of a LDPC code with the following parity check matrix $(\widetilde{\mathbf{H}})$ is depicted in Figure $4-4$. 


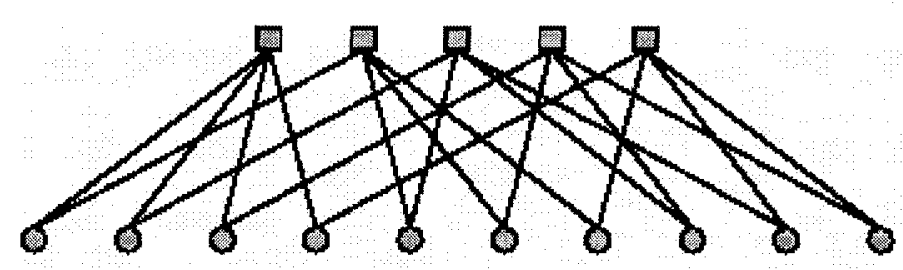

Figure 4-4. Tanner graph of the code with parity check matrix of $\widetilde{\mathbf{H}}$.

$$
\widetilde{\mathbf{H}}=\left[\begin{array}{llllllllll}
1 & 1 & 1 & 1 & 0 & 0 & 0 & 0 & 0 & 0 \\
1 & 0 & 0 & 0 & 1 & 1 & 1 & 0 & 0 & 0 \\
0 & 1 & 0 & 0 & 1 & 0 & 0 & 1 & 1 & 0 \\
0 & 0 & 1 & 0 & 0 & 1 & 0 & 1 & 1 & 0 \\
0 & 0 & 0 & 1 & 0 & 0 & 1 & 0 & 1 & 1
\end{array}\right]
$$

The parity check equations for this code are as follows:

$$
\begin{aligned}
& c_{1}+c_{2}+c_{3}+c_{4}=0, \\
& c_{1}+c_{5}+c_{6}+c_{7}=0, \\
& c_{2}+c_{5}+c_{8}+c_{9}=0, \\
& c_{3}+c_{6}+c_{8}+c_{9}=0, \\
& c_{4}+c_{7}+c_{9}+c_{10}=0 .
\end{aligned}
$$

In Figure 4-4 squares and circles represent the check nodes and the bit nodes respectively. The lines connecting these nodes show that each bit is involved in which parity check equations. For instance as presented in (4-15) the first, second, third and fourth bits are involved in the first parity check equation. It can also be confirmed that the first bit is involved in first and second parity check equations. A cycle in the Tanner graph is a path which starts and ends at the same bit node. Ideally, the Tanner graph should be cycle free which is not the case in practical systems.

The LDPC codes can be decoded with reasonable complexity by solving the parity check equations iteratively. The decoding is based on the Message Passing Algorithm 
(MPA) in which the LLRs are passed as message between the bit nodes and the check nodes iteratively. The MPA is based on the $\tanh$ rule. Let $C$ be the parity check of the bits $\left[c_{1} c_{2} \ldots c_{K_{0}}\right]$. If $\lambda_{C}$ and $\left[\lambda_{1} \lambda_{2} \ldots \lambda_{K_{0}}\right]$ represent the LLR of $C$ and the code bits respectively, it can be proven that the following equation should be satisfied [78]:

$$
\lambda_{C}=-2 \tanh ^{-1}\left(\prod_{i=1}^{K_{0}} \tanh \left(\frac{-\lambda_{i}}{2}\right)\right) .
$$

For cycle free codes, we have [78]:

$$
\lambda_{k}=\frac{2}{\sigma^{2}} r_{k}-2 \sum_{i=1}^{i_{c}} \tanh ^{-1}\left(\prod_{j=2}^{j_{c}} \tanh \left(\frac{-\lambda_{i, j}^{\prime}}{2}\right)\right)
$$

in which $\lambda_{i, j}^{\prime}$ is the LLR of the $j$-th bit node which is passed to the $i$-th check node. $\lambda_{i, j}^{\prime}$ can be computed recursively which makes it possible to decode the LDPC codes iteratively. The above algorithm which is based on the MAP criterion is known as the Belief Propagation (BP) algorithm. Equation (4-16) can also be expressed as:

$$
\lambda_{C}=-\prod_{i=1}^{K_{0}} \operatorname{sgn}\left(-\lambda_{i}\right) g\left(\sum_{i=1}^{K_{0}} g\left(\left|\lambda_{i}\right|\right)\right),
$$

where $g(x)=\ln \frac{e^{x}+1}{e^{x}-1}=-\ln (\tanh (x / 2))$.

It can be proven that $g(x)$ satisfies the following properties [78]:

- If $x>0$ then $g(x)>0$.

- $g(x)$ is monolithically decreasing such that $g(0)=\infty$ and $g(\infty)=0$.

- $g(g(x))=x$.

- If $\lambda_{1}<<\lambda_{2}$ then $g\left(\lambda_{1}\right)>>g\left(\lambda_{2}\right)$. 
Using the above properties, we can simplify and approximate (4-18) as:

$$
\sum_{i=1}^{K_{0}} g\left(\left|\lambda_{i}\right|\right) \approx g\left(\left|\lambda_{\min }\right|\right)
$$

where $\left|\lambda_{\min }\right|=\min \left\{\lambda_{1}|, \ldots,| \lambda_{K_{0}} \mid\right\}$. Therefore (4-18) can be simplified as:

$$
\lambda_{C} \approx-(-1)^{C}\left|\lambda_{\min }\right|
$$

The algorithm based on (4-20) is known as Min-Sum. Min-Sum is less complex than BP at the expense of some performance degradation [78].

\subsubsection{Performance comparison of TFDE with different coding methods}

In turbo systems by exchanging soft information we are able to improve the performance. Therefore both processors are required to be soft-input soft-output modules. As discussed in section 4.3.1, LDPC codes generate soft information in the form of LLR. Since the decoding of LDPC codes is performed iteratively, by using these codes in a turbo equalizer we have two types of iterations. The outer iterations are between the decoder and the equalizer. The inner iterations are in the LDPC decoder. We present the number of these iterations by $I_{o}$ and $I_{i}$ respectively. If we use a convolutional code, the conventional Viterbi algorithm is not useful unless we use Soft Output Viterbi Algorithm (SOVA) [79]. We can decode the convolutional code based on the MAP criterion by the BCJR algorithm which is slightly more complex than SOVA but achieves better performance [80][81][82]. Max-Log-Map is an approximation of the MAP which is less complex but effective. 
Figure 4-5 and Figure 4-6 present the performance of a TFDE using a LDPC code or a convolutional code in the form of BER and Frame Error Rate (FER). The codes used for simulations are a Recursive Systematic Convolutional (RSC) Code with the generator polynomial of $G(D)=\left(1, \frac{1+D^{2}}{1+D+D^{2}}\right)$ and a rate one half $(3,6)$ regular LDPC code with length of 1008 [83]. The FFT length is also 1008. In practical systems if the length is a power of two we can use efficient methods to perform the FFT. However even if the block length is not a power of two, there are still efficient algorithms to perform Fourier transform which are considerably less complex than the original method for DFT $[84][85][86][87]$. For instance the number of additions and multiplications required for Prime Factor Algorithm of [87] for $N=1008$, is almost the same as that of the Radix-two method for $N=1024$. The decoding algorithm for LDPC code is Min-Sum and for convolutional code is BCJR (Max-Log-MAP). The constellation is BPSK. The number of inner and outer iterations are $I_{i}=6$ and $I_{o}=4$ respectively. The interleaver used for the system with convolutional code is an S-Random interleaver which was first suggested in [88]. The bandwidth of the simulated system is $20 \mathrm{MHz}$. The channel is the 6-tap static channel whose parameters are presented in Table 4-1.

Table 4-1 Channel model

\begin{tabular}{|l|c|c|c|c|c|c|}
\hline Delay (nsec) & 0 & 310 & 710 & 1090 & 1730 & 2510 \\
\hline Power (dB) & 0 & -1 & -9 & -10 & -15 & -20 \\
\hline
\end{tabular}




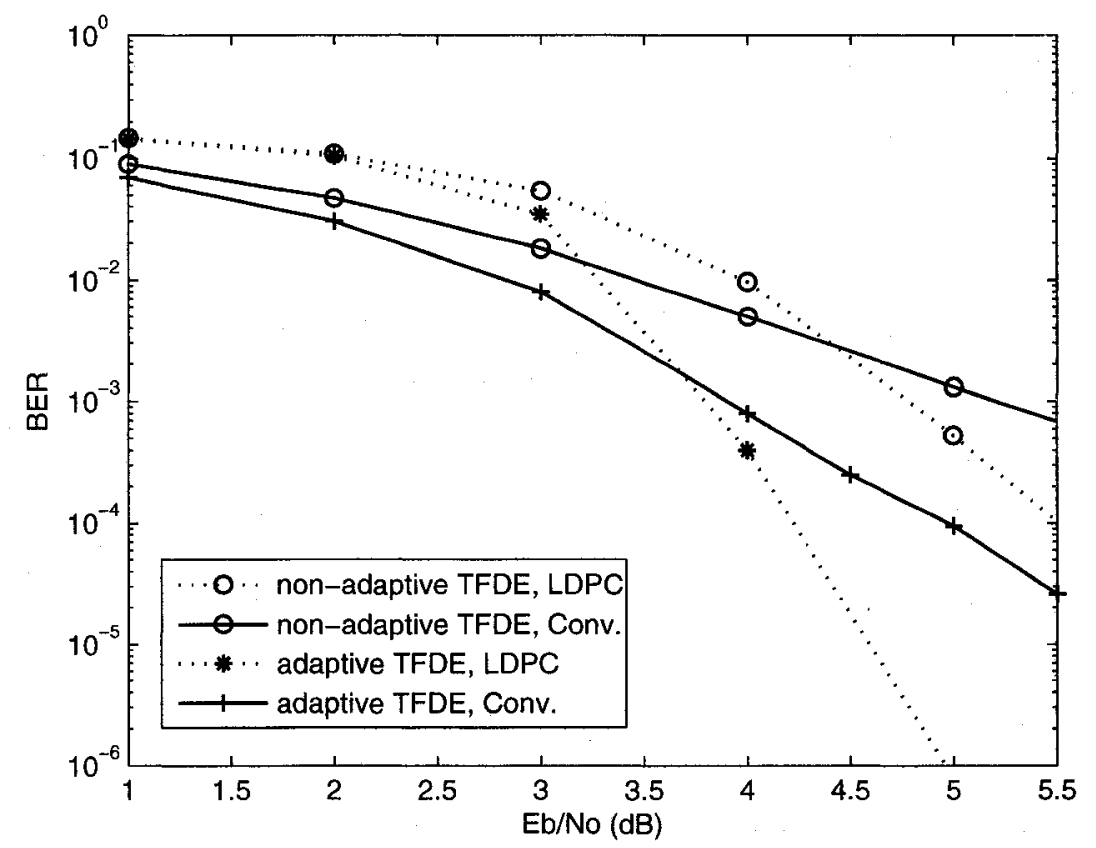

Figure 4-5. BER of TFDE with LDPC and Convolutional code, BPSK constellation.

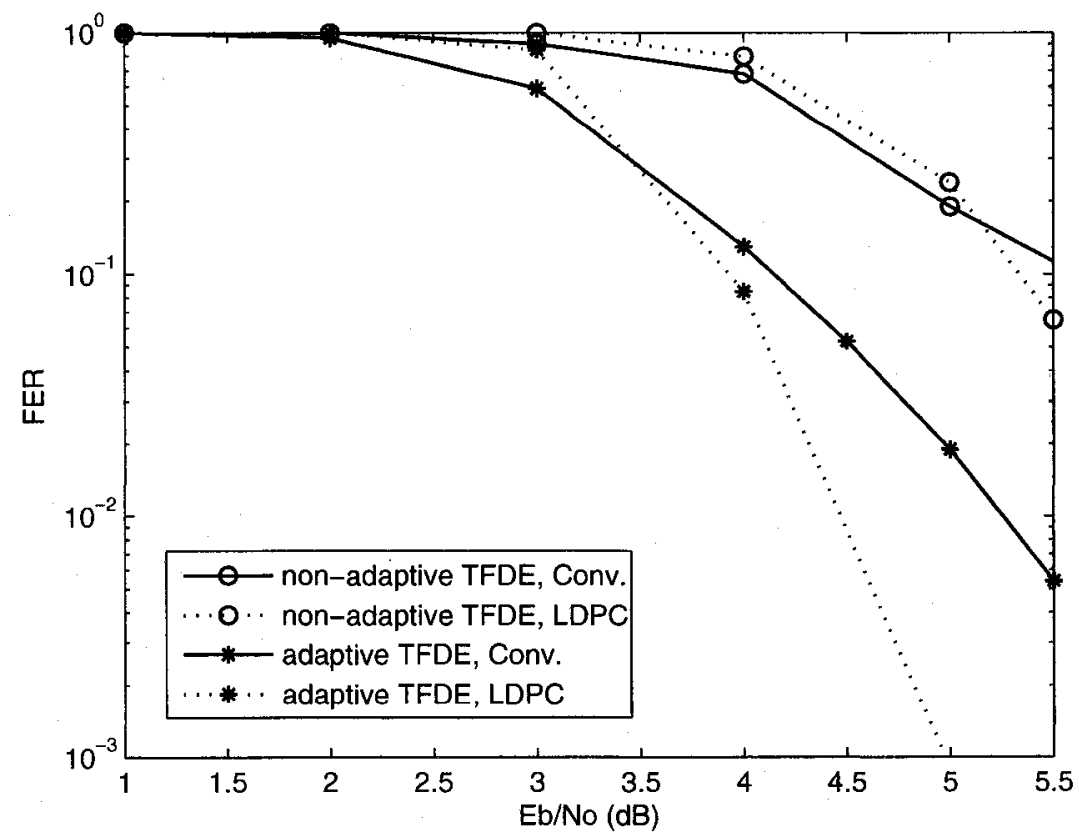

Figure 4-6. FER of TFDE with LDPC and Convolutional code, BPSK constellation. 
We consider both adaptive and non-adaptive methods discussed in sections 4.2 .1 and 4.2.2 respectively. As expected, the adaptive TFDE, in which the feedback information is used in designing the filter coefficients, performs better than non-adaptive TFDE. At a BER of $10^{-3}$ the adaptive method gives about $1.5 \mathrm{~dB}$ gain for the LDPC code and convolutional code compared to the non-adaptive method. The system with LDPC code can achieve considerably better performance than the one with convolutional code at high Signal to Noise Ratio (SNR) and it is worse in low SNR. In the desired range of bit error rate which is at most $10^{-4}$ the turbo equalizer with the LDPC code outperforms the one with the convolutional code.

\subsubsection{Complexity comparison of TFDE with different coding methods}

We compare the complexity of the decoding algorithms when Max-Log-Map and MinSum are used for decoding of convolutional and LDPC codes respectively. We also compare the overall complexity of the system when adaptive and non-adaptive equalizers are used. We assume that $N$ represents the code block length which is also assumed to be the FFT block length. The code rate is $R_{s}=\frac{1}{2}$. Let $m_{s}$ be the memory of the convolutional code and $N_{s}=2^{m_{s}}$ represent the number of states in the trellis diagram. We consider a $\left(i_{c} j_{c}\right)$ regular LDPC code. So $i_{c}$ and $j_{c}$ represent the number of ones in each column and each row of the parity check matrix respectively. Since the code rate is one half, $j_{c}=2 i_{c}$.

Table 4-2 presents the number of required additions, multiplications and comparisons for both decoders per block. Let $\alpha_{s}$ and $\beta_{s}$ be the metrics computed in forward and 
backward recursions and $\gamma_{s}$ be the metric on each branch of the convolutional code trellis diagram. The number of additions for computing $\alpha_{s}$ in the Max-Log-Map algorithm is $2 N_{s} N$. Computing $\beta_{s}$ requires the same number of additions. To determine $P(0)$ and $P(1)$ (the probability of the message bit being 0 or 1), $4 N_{s} N$ additions are needed. For $\gamma_{s}, 2 N$ additions and $4 N$ multiplications are required.

During each LDPC decoding iteration, all incoming messages to each bit-node and the channel message are added together, which requires $i_{c}$ additions. Now to generate the output message of each edge, we subtract the edge incoming message from the summation already computed. Thus for this stage the system needs $i_{c}$ additions. Therefore the total number of additions is $2 N\left(i_{c}+i_{c}\right)=4 i_{c} N$.

The total number of additions in the convolutional decoder increases exponentially with code memory while in the LDPC decoder it is proportional to the number of ones in the rows and columns of the parity check matrix which are small values. In both decoders, comparisons should be performed to find the maximum or minimum of some values.

Table 4-2 The Complexity of decoding algorithms

\begin{tabular}{|c|c|c|c|}
\hline Coding & \# of additions & \# of multiplications & \# of comparisons \\
& per block & per block & per block \\
\hline Max-Log-Map & $\left(8 \times 2^{m_{s}}+2\right) N$ & $4 N$ & $\left(4 \times 2^{m_{s}}-2\right) N$ \\
\hline Min-Sum & $I_{i} \times 4 i_{c} N$ & --- & $I_{i} \times j_{c} N$ \\
\hline
\end{tabular}


To determine $\alpha_{s}$ and $\beta_{s}$ in Max-Log-Map, we need $2 N_{s} N$ comparisons and to find $P(0)$ and $P(1)$, we need $2\left(N_{s}-1\right) N$ comparisons. To update the value for the checknodes in LDPC, $N I_{i} j_{c}$ comparisons should be performed where $I_{i}$ is the number of iterations in LDPC decoder.

For the decoder parameters used in section 4.3.2, the number of additions per block would be $34 N$ and $72 N$ for the convolutional code and LDPC code respectively. The number of multiplications in the convolutional code would be $4 N$ while the LDPC code does not require any multiplication. The number of comparisons is $14 \mathrm{~N}$ and $36 \mathrm{~N}$ for BCJR and LDPC respectively. As can be seen the complexity of both decoding methods are almost comparable while the system achieves better performance if LDPC is used.

For comparison of system overall complexity we should also consider the equalizer part. Calculating non-adaptive equalizer coefficients requires $2 N+1$ multiplications and $N$ additions. In adaptive TFDE, to calculate $v$ we need $N+1$ additions and $N+1$ multiplications. To generate the filter coefficients $3 N$ multiplications and $N$ additions are needed. Each iteration contains two FFT/IFFT, $N$ additions for computing $R_{k}-\bar{R}_{k}$ and $N$ additions for adding the biasing term $\mu \bar{X}_{k}$. Frequency domain equalization includes $N$ multiplications in each iteration. The same number of multiplications is required for generating the interference signal in the feedback loop. $N$ additions and $N$ multiplications are required during computing of $\mu$. In the non-adaptive TFDE, $\mu$ is computed once for each block while in the adaptive TFDE, $\mu$ should be updated during iterations. Prior to the loop there is another FFT. To compute the frequency response of the channel another FFT is required. The overall complexity of both adaptive and non-adaptive methods is 
presented in Table 4-3 where $I_{o}$ represents the number of outer iterations between the equalizer and decoder.

As depicted in Table 4-3, adaptive TFDE is only slightly more complex. Therefore the complexity of both methods is almost the same while adaptive TFDE achieves better performance.

\subsection{Conclusion}

In this chapter we considered adaptive and non-adaptive TFDE. In non-adaptive TFDE the FDE coefficients are fixed while in adaptive TFDE, they change based on the decoder output information as iterations continue. We also considered two coding methods, convolutional and LDPC codes. We compared these four cases in terms of performance and complexity. The system achieves the best performance when the equalizer is adaptive and the LDPC code is used, while its complexity is not considerably higher than the other cases.

Table 4-3 Overall complexity of adaptive and non-adaptive TFDE

\begin{tabular}{|c|c|c|}
\hline & $\begin{array}{c}\text { Number of complex } \\
\text { multiplications per block }\end{array}$ & $\begin{array}{c}\text { Number of complex } \\
\text { additions per block }\end{array}$ \\
\hline Non-adaptive & $N\left[\left(I_{o}+1\right) \log _{2} N+2 I_{o}+3\right]+1$ & $N\left[\left(2 I_{o}+2\right) \log _{2} N+2 I_{o}+2\right]$ \\
\hline Adaptive & $N\left[\left(I_{o}+1\right) \log _{2} N+7 I_{o}\right]+I_{o}$ & $N\left[\left(2 I_{o}+2\right) \log _{2} N+5 I_{o}\right]+I_{0}$ \\
\hline
\end{tabular}




\section{Chapter 5 BERT chart analysis of TFDE}

\subsection{Introduction}

Different analysis tools have been proposed to evaluate the performance of iterative systems including turbo equalization [53][54][55] [56][89][90]. These methods avoid the extensive Monte Carlo simulations required to determine the system performance. They also simplify the system design when different schemes for the equalizer or decoder are available. These methods are based on tracking a single parameter whose computation is based on the density of the bit log-likelihood ratio at the output of the SISO processor (decoder or equalizer) throughout iterations. The EXIT chart is regarded as the most well- 
known tool to analyze a turbo system. It was first proposed in [53][54] as a tool for the convergence analysis of turbo codes and LDPC codes and then was used for other turbo based systems as well as turbo equalization [55]. The EXIT chart evaluates the convergence of the system by tracking the mutual information exchanged between the two processing blocks. Other parameters have been considered to be tracked throughout iterations such as signal to noise ratio [90] and bit error rate [56][91]. Among these methods, the EXIT chart method has shown to result in more accurate prediction [92].

In this thesis, we use an alternative method to the EXIT chart, known as the BERT chart to analyze the performance and convergence behavior of a turbo equalizer. The BERT chart method, similar to the EXIT chart, includes two curves. Each curve represents the BER at the output of the SISO processor as a function of the input BER. In this thesis, to generate the equalizer BER curve we analytically obtain the BER at the equalizer output based on the decoder BER at the previous iteration, channel parameters and noise variance. Compared to the histogram based method of generating the EXIT chart for an equalizer in [55], this method is much easier to implement, especially for the case of non-static channels and varying channel signal to noise ratios. The decoder BER curve is its performance curve in the additive white Gaussian noise channel which has to be generated from decoder analysis or simulations in AWGN.

The BERT chart analysis was initially suggested in [56] for time domain turbo equalization. In this thesis, we employ the BERT chart method for the first time for a TFDE. In [56] the equalizer curve has been obtained by interpolating between only two boundary points. For the first point, we determine the equalizer output BER when there is no a priori information available from the decoder, which happens in the first iteration. 
The second point represents the case with perfect a priori information. In our method, we analytically determine the equalizer output mean and variance for any a priori information generated by the decoder including the two boundary points. It is shown in [55] that for MMSE turbo equalizers, the probability density of the equalizer output is very well approximated by a Gaussian variable. Therefore, with the mean and variance of the equalizer output being available, the density of the equalizer output is fully described. We then compute the associated bit error rate for this Gaussian variable for a range of input bit error rates to generate the equalizer BER curve ${ }^{10}$. Although the method in [56] results in reasonably good predictions for a large enough number of iterations, our method is more accurate especially when it is used to predict the performance in intermediate iterations (for instance, the performance in the second iteration in a system with ten iterations).

Turbo equalizers can be adaptive or non-adaptive during iterations. In [56] only the latter case was considered. We extend our method to adaptive turbo equalizers where for each iteration we have a new equalizer curve. Such systems would be too difficult to analyze using the histogram based EXIT chart method of [55]. We also consider other constellations in addition to BPSK which was considered in [56] for a turbo time domain equalizer. We will show that the difference between the prediction accuracy of the proposed BERT chart for TFDE and the method of [56] is more significant for higher order constellations and for the adaptive case.

\footnotetext{
${ }^{10}$ We note that when we determine the mean and variance of this Gaussian density, we could track the associated mutual information instead of BER and generate the EXIT curve analytically (not histogram based). However, since there is a one to one mapping between the mutual information and probability of error of a Gaussian variable, the results would be the same. We choose to track BER since it provides more insight in the convergence region as discussed in section III-C of [56].
} 
The EXIT charts produced in [55] (for both decoder and equalizer) are generated using large block lengths to analyze the asymptotic performance of turbo equalization. They could effectively predict the performance when the interleaver size is large enough (e.g., order of $10^{5}$ and above). For systems with short block lengths (e.g., 10 ${ }^{3}$ ), the actual Monte Carlo performance will usually be considerably worse (e.g., $2 \mathrm{~dB}$ ) than the predicted value by the asymptotic EXIT chart method. One advantage of the proposed method is to use the actual finite length BERT curve for the decoder instead of using the asymptotic decoder EXIT curve in [55]. This fact makes it possible to predict the performance of a TFDE system with short block length more accurately. Depending on the modulation type, our results were only $0.1 \mathrm{~dB}$ to $0.25 \mathrm{~dB}$ away from the actual Monte Carlo simulation performance for a block length of 1008 .

\subsection{Bit Error Rate Transfer Chart}

The purpose, toward which this work is directed, is to predict the performance of TFDE by available knowledge about the channel characteristics and encoder/decoder parameters. As the initial step to achieve this goal, we first turn our attention to a simplified receiver structure that only contains a frequency domain equalizer without decoding or feedback loop and then generalize our approach to TFDE. To determine the equalizer BER, we apply the widely used assumption of approximating the equalizer output by a Gaussian distributed random variable. The equalizer output can be estimated as: 


$$
z_{n}=\alpha x_{n}+\eta_{n},
$$

where $\alpha x_{n}$ is the part associated with the desired symbol and $\eta_{n}$ is the noise term including the residual ISI and channel noise. It can be easily shown that [93][94]:

$$
\alpha=\frac{1}{N} \sum_{k=1}^{N} \frac{\left|H_{k}\right|^{2}}{\frac{\sigma^{2}}{\sigma_{d}^{2}}+\left|H_{k}\right|^{2}} .
$$

By comparing (5-2) and (4-14), we conclude that $\alpha=\mu$. Therefore for a FDE without feedback information, it can be easily proved that the conditional mean of the random variable $z_{n}$ is $\mu$ and the variance is:

$$
\Sigma_{0}^{2}=E\left(|\eta|^{2}\right)=\sigma_{d}^{2}\left(\mu-\mu^{2}\right)
$$

Considering the Gaussian approximation, we can find the equalizer output BER similar to BER of an AWGN channel when mean and variance of the channel output are equal to $\mu$ and $\Sigma_{0}^{2}$ respectively. For BPSK the equalizer BER would be:

$$
P_{E q}=Q\left(\frac{\mu}{\Sigma_{0}}\right)
$$

Assuming Gray mapping and one bit error per each symbol error, the BER for 16-QAM can be approximated as: 


$$
P_{E q}=\frac{1}{4}\left(3 Q_{1}-2 Q_{3}\right)\left(1-Q_{3}\right),
$$

where $Q_{1}=Q\left(\frac{\sqrt{2} \mu}{\Sigma_{0}}\right)$ and $Q_{3}=Q\left(\frac{3 \sqrt{2} \mu}{\Sigma_{0}}\right)$. To evaluate the accuracy of this Gaussian approximation, in Figure 5-1 the simulation results and the results of using (5-4) and (5-5) are presented which verifies the accuracy of the Gaussian assumption. The channel model is presented in Table 4-1.

We can generalize this approach to a turbo equalizer where the equalizer is followed by the decoder and feedback loop. In the following two subsections we use this approach for both non-adaptive and adaptive TFDE.

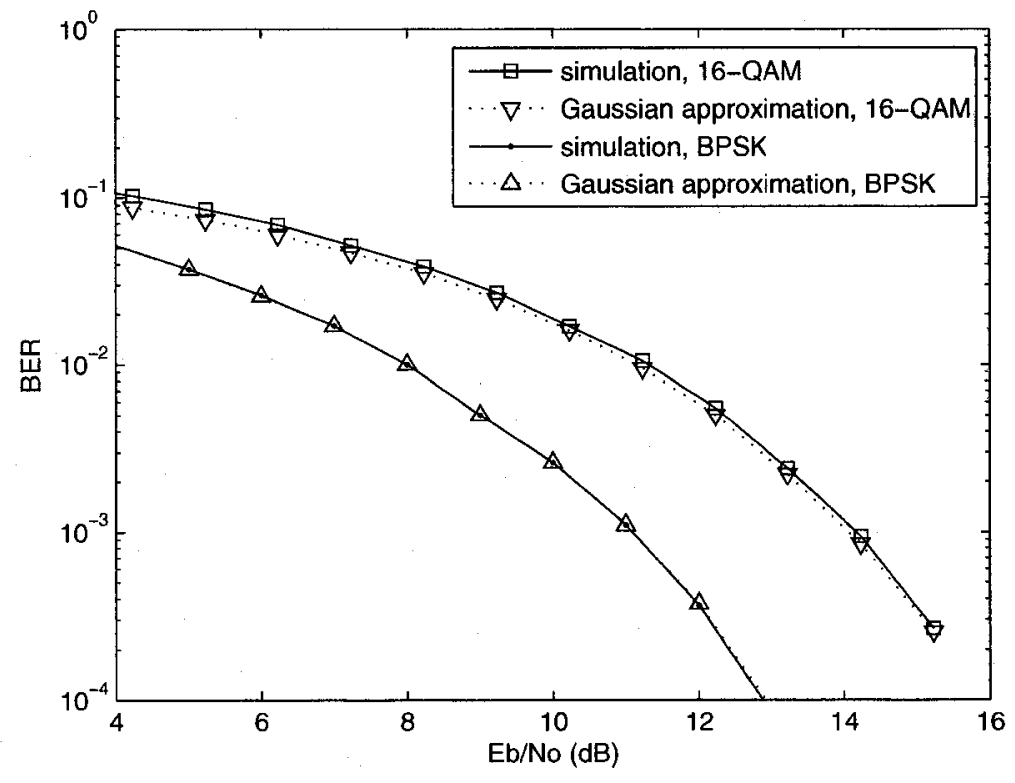

Figure 5-1. Comparison of Gaussian Approximation and simulation results for a FDE. 


\subsubsection{BERT chart for non-adaptive TFDE}

\subsubsection{BPSK Constellation}

In the first iteration, the receiver is a linear FDE followed by the decoder with no a priori information. Therefore (5-4) can be applied to find the equalizer bit error rate. Since the equalizer output is approximated by a Gaussian random variable, we can use the decoder performance curve in an AWGN channel to obtain the decoder output BER. To use this curve, we can find the corresponding equalizer equivalent SNR, the ratio of mean squared to the variance of equalizer output, according to the BER of an uncoded system with a Gaussian channel and use the $\mathrm{SNR}_{\mathrm{in}}-\mathrm{BER}_{\text {out }}$ decoder curve. Equivalently, we can find the equalizer output BER and convert the decoder curve from $\mathrm{SNR}_{\mathrm{in}}-\mathrm{BER}_{\text {out }}$ to $\mathrm{BER}_{\mathrm{in}}-\mathrm{BER}_{\text {out. }}$ Using $\mathrm{BER}_{\mathrm{in}}-\mathrm{BER}_{\mathrm{out}}$ is more convenient for higher order constellations. Converting $\mathrm{SNR}_{\text {in }}$ to $\mathrm{BER}_{\text {in }}$ is performed by $B E R_{\text {in }}=Q\left(\sqrt{S N R_{\text {in }}}\right)$ where $\mathrm{SNR}_{\text {in }}$ is at the decoder input. In other iterations, the variance of the equalizer output in the $i$-th iteration $\left(\Sigma_{i}^{2}\right)$ depends on the decoder output in the (i-1)-th iteration. As presented in Appendix D, the equalizer output variance can be calculated as:

$$
\begin{aligned}
\Sigma_{i}^{2}= & \sigma_{d}^{2} \rho+\sigma^{2} \zeta-\sigma_{d}^{2}|\mu|^{2} \\
& +\frac{1}{N} \sum_{k=1}^{N} H_{k}^{*} W_{k}^{*}\left(\mu-H_{k} W_{k}\right) E^{i-1}\left(\bar{x}_{n} x_{n}^{*}\right) \\
& +\frac{1}{N} \sum_{k=1}^{N} H_{k} W_{k}\left(\mu-H_{k} W_{k}\right)^{*} E^{i-1}\left(\bar{x}_{n}^{*} x_{n}\right) \\
& +\frac{1}{N} \sum_{k=1}^{N}\left|\mu-H_{k} W_{k}\right|^{2} E^{i-1}\left(\left|\bar{x}_{n}\right|^{2}\right) .
\end{aligned}
$$

where $H_{k}$ and $W_{k}$ are the $k$-th component of channel frequency response and frequency domain equalizer respectively. $\bar{x}_{n}$ is the $n$-th soft symbol generated in the feedback loop 
and $E^{i}($.$) denotes the expectation in the i$-th iteration, $\zeta=\frac{1}{N} \sum_{k=1}^{N}\left|W_{k}\right|^{2}$ and $\rho=\frac{1}{N} \sum_{k=1}^{N}\left|H_{k} W_{k}\right|^{2}$

To determine $\Sigma_{i}^{2}$, we should find the distribution of the soft symbols used in the feedback loop which depends on the decoder output LLR distribution according to (4-6). The LLR conditional distribution can be well approximated as Gaussian [95]. As a result, the probability density function (pdf) of LLR in the $i$-th iteration can be expressed as:

$$
f_{\lambda}^{i}(\lambda)=\frac{1}{2 \sqrt{4 \pi v_{i}^{2}}}\left[\exp \left(\frac{-\left(\lambda-\left|m_{i}\right|\right)^{2}}{2 v_{i}^{2}}\right)+\exp \left(\frac{-\left(\lambda+\left|m_{i}\right|\right)^{2}}{2 v_{i}^{2}}\right)\right]
$$

where $m_{i}$ and $v_{i}^{2}$ are the conditional mean and variance in the $i$-th iteration. The value of $m_{i}$ and $v_{i}^{2}$ are determined based on the decoder error probability. It has been shown that the distribution of the conditioned LLR has the consistency property [95]. For Gaussian random variables this means the variance is twice the absolute value of the mean $\left(v_{i}^{2}=2\left|m_{i}\right|\right)$. Therefore one parameter $\left(m_{i}\right)$ is enough to describe the Gaussian distribution. This assumption has been already used in [55][54] and the analytical proof and more details can be found in [95]. Considering the consistency property of LLR, the decoder bit error rate in the $i$-th iteration $\left(P_{i}\right)$ can be expressed as:

$$
P_{i}=\frac{1}{2} P\left\{\lambda_{n}<0 \mid c_{n, 1}=0\right\}+\frac{1}{2} P\left\{\lambda_{n}>0 \mid c_{n, 1}=1\right\}=P\left\{\lambda_{n}<0 \mid c_{n, 1}=0\right\}=Q\left(\sqrt{\frac{\left|m_{i}\right|}{2}}\right)
$$

Therefore the mean of this random variable would be: 


$$
\left|m_{i}\right|=2\left[Q^{-1}\left(P_{i}\right)\right]^{2}
$$

Based on this assumption and using (4-6), the distribution of the soft symbols $(\bar{x})$ conditioned on the transmitted bit would be [96]:

$$
f_{\bar{x}}^{i}(\bar{x} \mid x)=\frac{2 g_{1}\left[\ln \left(\frac{1+\bar{x}}{1-\bar{x}}\right)\right]}{\left(1-\bar{x}^{2}\right)}
$$

where

$$
g_{1}(z)=\frac{1}{\sqrt{4 \pi\left|m_{i}\right|}} \exp \left(\frac{\left(z-(-1)^{x} m_{i}\right)^{2}}{4\left|m_{i}\right|}\right)
$$

If we represent $E^{i}\left(\bar{x}_{n} \mid c_{n, k}=b\right)$ by $E^{i}\left(\bar{x}_{n} \mid b\right)$, it can be easily shown that: $E^{i}\left(\bar{x}_{n} x_{n}\right)=E^{i}\left(\bar{x}_{n} \mid 0\right), E^{i}\left(\bar{x}_{n}{ }^{2}\right)=E^{i}\left(\bar{x}_{n}{ }^{2} \mid 0\right)$. Using (5-10) it can also be proven that $E^{i}\left(\bar{x}_{n}{ }^{2} \mid 0\right)=E^{i}\left(\bar{x}_{n} \mid 0\right)$. The value of $E^{i}\left(\bar{x}_{n}{ }^{2}\right)$ depends on $m_{i}$ and consequently is a function of $P_{i}$. If we define $\Delta_{i}$ as:

$$
\Delta_{i} \stackrel{\Delta}{=} E^{i}\left(\tanh \left(\frac{\lambda}{2}\right) \mid 0\right)=E^{i}\left(\left[\tanh \left(\frac{\lambda}{2}\right)\right]^{2} \mid 0\right)
$$

for BPSK, $E^{i}\left(x_{n}^{2}\right)=\Delta_{i}$ and therefore (5-6) is simplified to:

$$
\Sigma_{i}^{2}=\sigma^{2} \zeta+\left(\rho-|\mu|^{2}\right)\left(\sigma_{d}^{2}-\Delta_{i-1}\right)
$$

We can summarize the steps of the BERT chart algorithm as follows:

(1) Compute $\mu$ and $\Sigma_{0}^{2}$ after iteration 0 from (4-14) and (5-3). 
(2) Compute the equalizer output BER $\left(P_{E q}\right)$ for the $i$-th iteration (from (5-4) for BPSK and from (5-5) for 16-QAM for iteration $0, \Sigma_{0}^{2}$ should be replaced by $\Sigma_{i}^{2}$ for $i$-th iteration).

(3) From the decoder empirical $\mathrm{BER}_{\mathrm{in}}-\mathrm{BER}_{\text {out }}$ curve find the corresponding decoder output BER $\left(P_{i}\right)$.

(4) Find the corresponding $m_{i}$ from (5-9).

(5) Calculate $\Delta_{i}=E^{i}\left(\bar{x}_{n}^{2}\right)$ using (5-10).

(6) Compute $\Sigma_{i+1}^{2}$ from (5-6) (simplified to (5-13) for BPSK).

(7) Go to step (2).

Equivalently we can follow these steps on the BERT chart. This chart contains two curves as presented in Figure 5-2 for BPSK. One of them is the decoder curve representing its output BER versus its input BER in the AWGN channel. Since the decoder input BER is the same as equalizer output BER $\left(P_{E q}\right)$, in each iteration we can find the corresponding $P_{i}$ using the decoder curve. This curve is fixed for a given code because it only depends on the code structure, decoding algorithm and block length. The other curve in Figure 5-2 is for the equalizer which gives $P_{E q}$ as a function of decoder BER in the previous iteration $\left(P_{i-1}\right)$. This curve depends on the channel parameters and the noise power, so it changes according to the signal to noise ratio for which we are analyzing the system performance. The trajectories between the two curves follow the aforementioned steps and represent what occurs as iterations continue. 


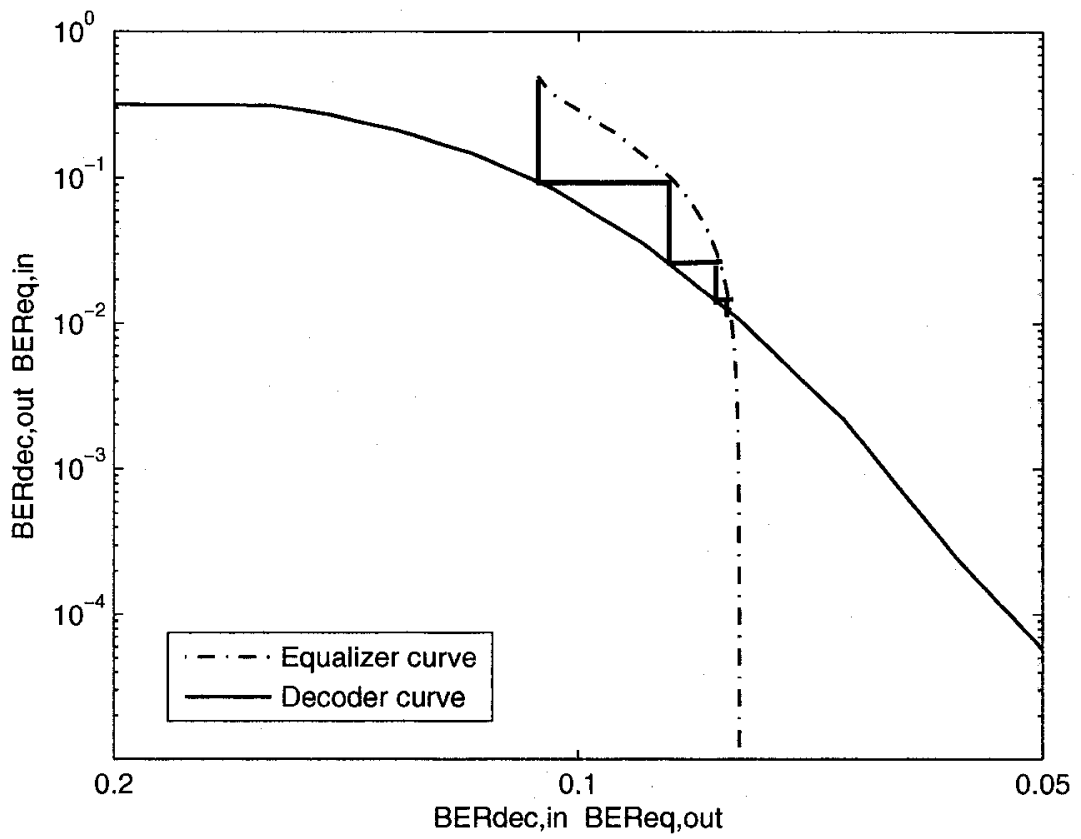

Figure 5-2. BERT chart of non-adaptive TFDE for BPSK constellation at $E b / \mathbf{N}_{0}=4 \mathrm{~dB}$. 


\subsubsection{16-QAM Constellation}

The basic idea of the BERT chart analysis for 16-QAM and BPSK is similar. We first calculate the equalizer output variance $\left(\Sigma_{i}^{2}\right)$ and then evaluate its BER from (5-5). As in the BPSK case, we can find $\Sigma_{i}^{2}$ using (5-6) noting that $E^{i}\left(\bar{x}_{n}{ }^{2}\right), E^{i}\left(\bar{x}_{n}^{*} x_{n}\right)$ and $E^{i}\left(\bar{x}_{n} x_{n}^{*}\right)$ would differ from the case of BPSK. As proven in Appendix E, all these expectations have similar values which are equal to:

$$
E^{i}\left(\bar{x}_{n} \bar{x}_{n}^{*}\right)=E^{i}\left(x_{n} \bar{x}_{n}^{*}\right)=E^{i}\left(x_{n}^{*} \bar{x}_{n}\right)=8 \Delta_{i}+2 \Delta_{i}^{2},
$$

where $\Delta_{i}$ has been already defined in (5-12). Therefore the equalizer output variance in the $i$-th iteration is:

$$
\Sigma_{i}^{2}=\sigma^{2} \zeta+\left(\rho-|\mu|^{2}\right)\left(10-8 \Delta_{i-1}-2 \Delta_{i-1}^{2}\right)
$$

Now we can repeat all the seven steps, described in the previous section, to evaluate the system performance.

\subsubsection{BERT chart for adaptive TFDE}

For a TFDE system with an adaptive equalizer which is not fixed during iterations, we can still use (5-13) to find the equalizer output variance. However, as $W_{k}$ changes during iterations, the mean and variance change accordingly. So even for a fixed signal to noise ratio, the equalizer curve in the BERT chart is not fixed during iterations. As presented in 
(4-12), $W_{k}$ depends on $v$ which is the variance of the feedback symbols ${ }^{11}$. Although in the BERT chart analysis the soft symbols $\left(\bar{x}_{n}\right)$ are not available, we can estimate $v$ if we replace $\frac{1}{N} \sum_{n=1}^{N}\left|\bar{x}_{n}\right|^{2}$ by $E\left(\left|\bar{x}_{n}\right|^{2}\right)$. Hence for BPSK, the equalizer output variance for the $i$-th iteration is:

$$
v=1-E^{i-1}\left(\left|\bar{x}_{n}\right|^{2}\right)=1-\Delta_{i-1}
$$

Similarly for 16-QAM, $v$ can be approximated as:

$$
v=10-\left(8 \Delta_{i-1}+2 \Delta_{i-1}^{2}\right) .
$$

Thus we can use the previous steps in section 5.2.1.1 while the sixth step will change into three sub-steps as follows:

(6.1) Find $v$ from (5-16) or (5-17) for BPSK or 16-QAM respectively.

(6.2) Find new equalizer coefficients from (4-12).

(6.3) Find $\mu_{i}$ from (4-14) and calculate $\Sigma_{i}^{2}$ from (5-13) and (5-15) for BPSK and 16QAM.

\subsection{Simulation Results}

We consider a TFDE using an LDPC code. The code is a rate one half regular $(3,6)$ LDPC code with block length of $N=1008$. The number of inner iterations $\left(I_{i}\right)$ is 6 and the

\footnotetext{
${ }^{11}$ In the simulations $v$ is calculated by $v=E\left(x_{n} x_{n}^{*}\right)-\frac{1}{N} \sum_{n=1}^{N}\left|\bar{x}_{n}\right|^{2}$.
} 


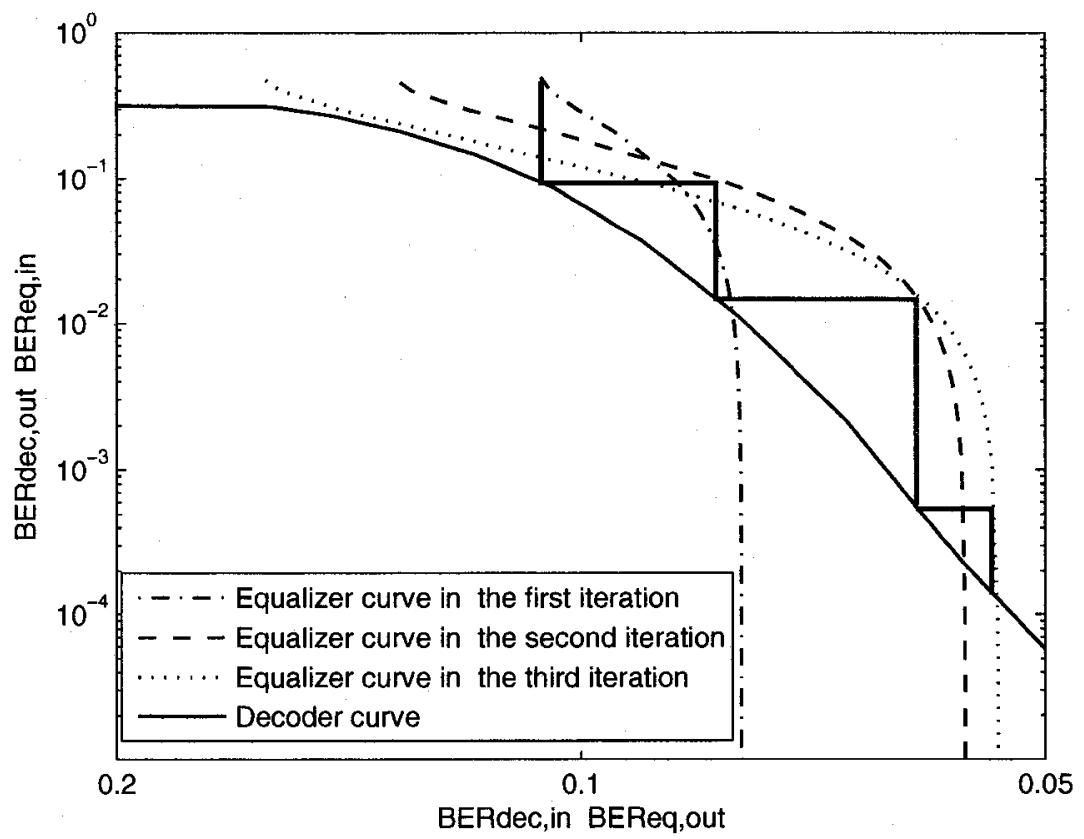

Figure 5-3. BERT chart of adaptive TFDE for BPSK constellation at $\mathbf{E b} / \mathbf{N}_{0}=4 \mathrm{~dB}$. 
number of outer iterations ( $I_{o}$ ) is 4. We consider both BPSK and 16-QAM constellations. The channel is defined in Table 4-1. The FFT size is 1008.

Figure 5-2 and Figure 5-3 depict the BERT chart for non-adaptive and adaptive TFDE respectively when the constellation is BPSK and $\mathrm{Eb} / \mathrm{N}_{0}=4 \mathrm{~dB}$. In the first iteration, the equalizer curve is the same in both figures. In the adaptive TFDE, there is a new equalizer curve for each iteration and the trajectories follow the corresponding equalizer curve as iterations continue. If we compare the $i$-th and $(i+1)$-th equalizer curves, we find out that for the given decoder BER in the $i$-th iteration, the $(i+1)$-th equalizer curve achieves a smaller BER than that of the $i$-th equalizer curve. Therefore by using the adaptive TFDE we can achieve better performance for the same signal to noise ratio.

Figure 5-4 and Figure 5-5 present the BERT chart results and simulation results after four iterations between the equalizer and decoder for BPSK and 16-QAM respectively ${ }^{12}$. As confirmed by the figures the performance predicted by the BERT chart is close to the simulation results. For instance for BPSK constellation it is about $0.1 \mathrm{~dB}$ away from the simulation result for non-adaptive TFDE and at most $0.2 \mathrm{~dB}$ away for the adaptive case. As can be seen in Figure 5-5, the BERT chart method is also accurate for 16-QAM. For the non-adaptive case there is at most $0.2 \mathrm{~dB}$ difference with simulation results. This value increases to $0.25 \mathrm{~dB}$ for adaptive TFDE.

\footnotetext{
${ }^{12}$ The BERT chart results for WINNER channel C2 is also presented in Appendix F. The channel C2 parameters are presented in Table F-1.
} 


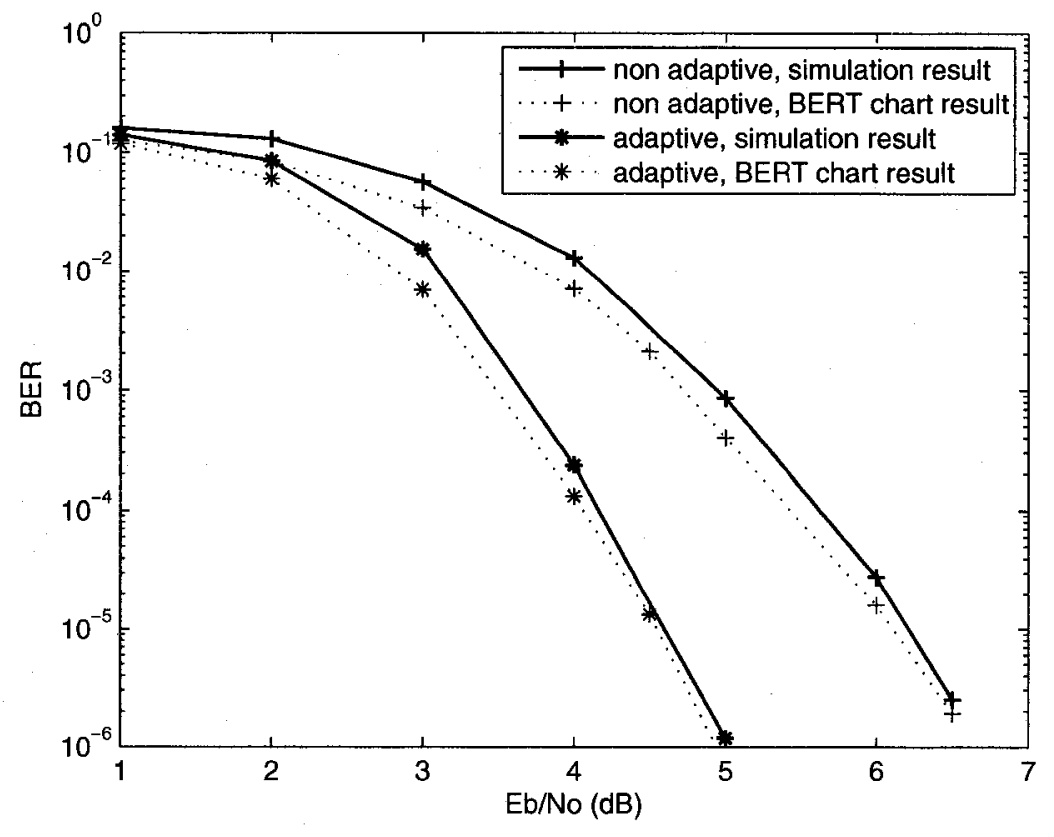

Figure 5-4. Comparison of BERT chart result and simulation result, BPSK.

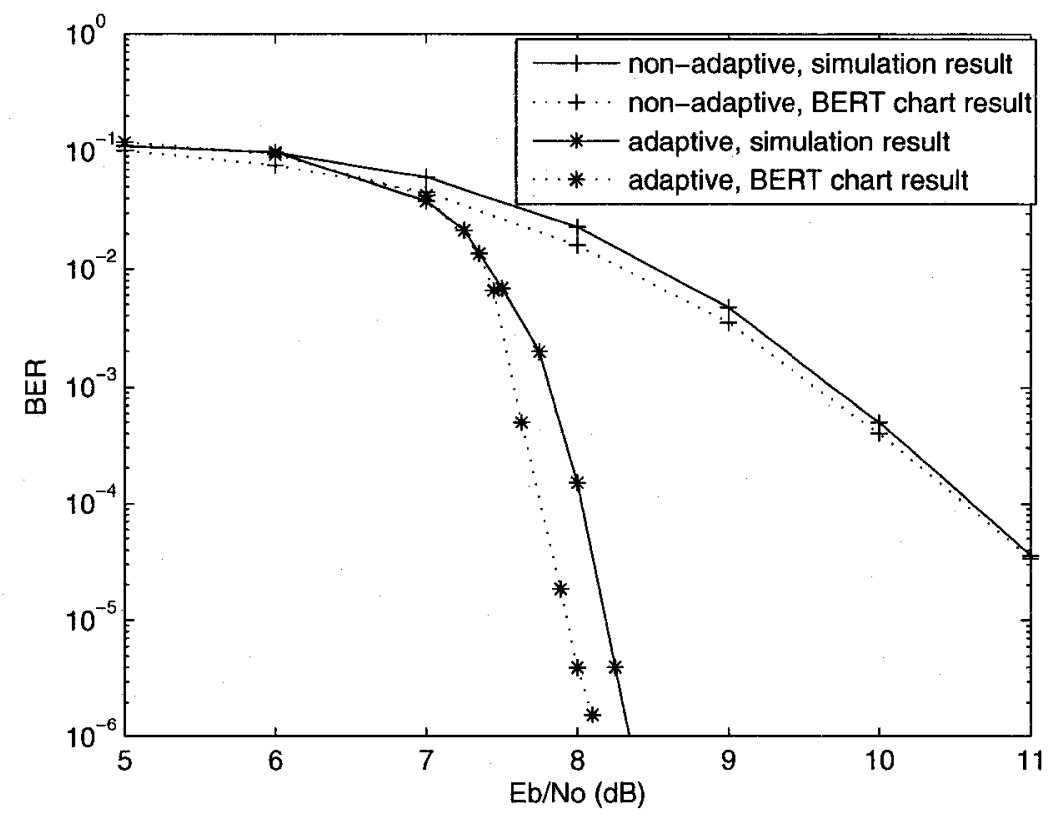

Figure 5-5. Comparison of BERT chart result and simulation result, 16-QAM. 


\subsection{Comparison with other analysis tools}

\subsubsection{Previously proposed BERT chart}

As discussed in section 5.1, although the basic idea of the BERT chart in this thesis and the previous one in [56] is similar, the derivation of the equalizer curve is different. In [56], the equalizer BER is obtained for two boundary points that correspond to $P_{i}=0.5$ and $P_{i}=0$ where $P_{i}$ is the decoder BER in the previous iteration. To find the equalizer bit error rate, a summation over all interfering components is calculated.

Therefore the complexity increases exponentially with the memory of the overall channel (the convolution of the ISI channel and equalizer transfer function). The other points of the equalizer curve are found by linear interpolation between these two points.

In our method, due to the structure of frequency domain equalization, we are able to find the BER for any value of $P_{i}\left(0<P_{i}<0.5\right)$. In this process the parameters $\mu, \rho$ and $\zeta$ should be calculated once for each equalizer curve. The complexity of this calculation increases linearly with the block length. Since by this method we are able to find all points of the curve, it is expected to be more accurate especially for higher order constellations and adaptive TFDE. Because of the waterfall form of the equalizer curve, usually after a large number of iterations, the results of both methods are fairly similar. The difference is more significant when predicting the performance in intermediate iterations. Figure 5-6 illustrates the performance of both methods in the second iteration for an adaptive TFDE with 16-QAM symbols over the channel presented in Table 4-1. Figure 5-7 shows the performance of a non-adaptive TFDE with BPSK in the second 


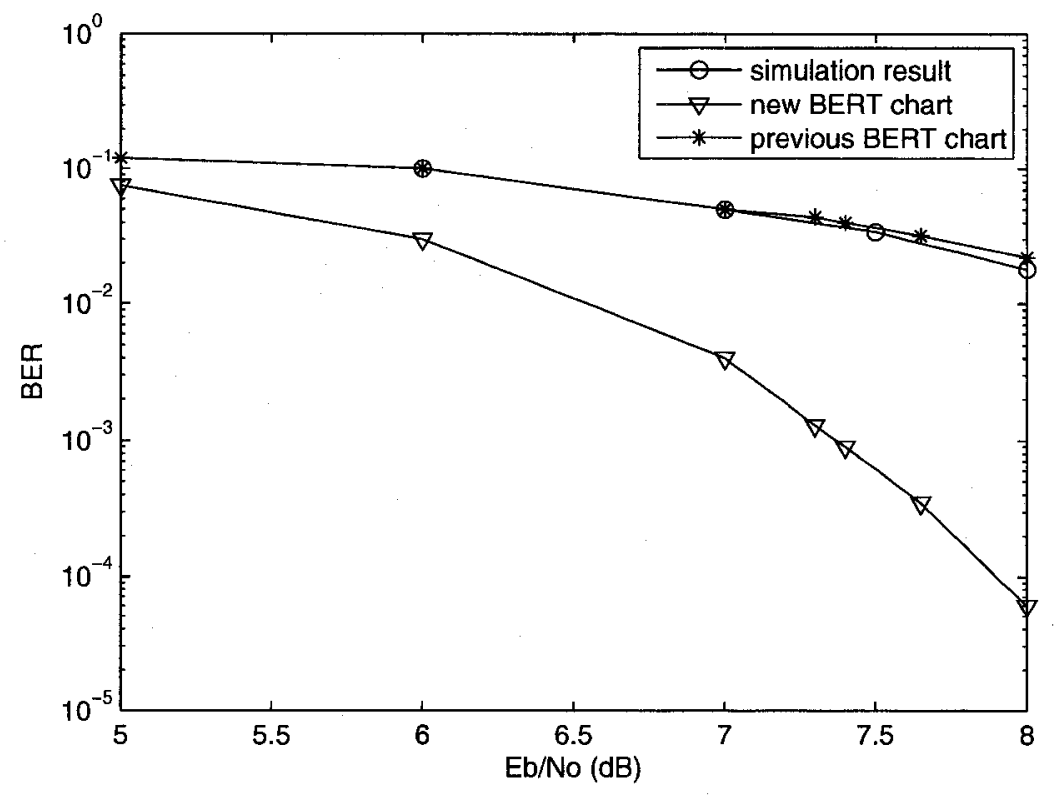

Figure 5-6. Comparison between the previous (as in [56]) and proposed BERT chart, second iteration, adaptive TFDE, 16-QAM, over channel described in Table 4-1.

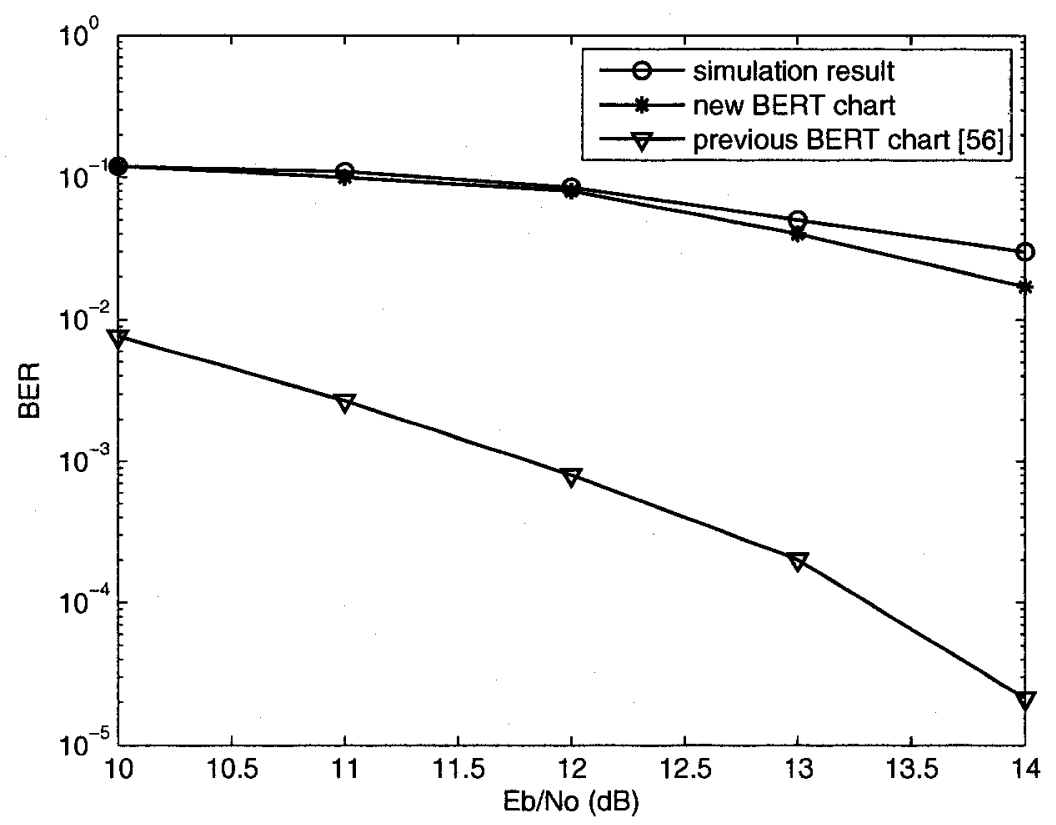

Figure 5-7. Comparison between the previous (as in [56]) and proposed BERT chart, second iteration, non-adaptive TFDE, BPSK, over Proakis channel C. 
iteration over the Proakis channel C [1]. As shown in these figures, the new method provides a considerably more accurate prediction than the previous one.

\subsubsection{EXIT chart}

In this section, we compare our method with the EXIT chart method of [55]. In all histogram based methods including that of [55], for each SISO processor, a very large number (e.g., $10^{7}$ ) of coded bits and the corresponding a priori LLRs have to be generated.

The conditional pdf of the output LLRs $(f(l \mid c=b)$, where $b \in\{0,1\}, l$ can be replaced by $\lambda$ or $\left.\lambda^{\prime}\right)$ is then estimated. To generate the EXIT chart, (5-18) can be used to obtain the output mutual information $\left(I_{o}\right)$.

$$
I_{o}=\frac{1}{2} \sum_{c \in S} \int_{-\infty}^{+\infty} f(l \mid c) \log _{2} \frac{2 f(l \mid c)}{f(l \mid c=-1)+f(l \mid c=+1)} d l
$$

At the input of the next SISO processor, the computed pdf is approximated by a Gaussian variable with the same mutual information [96]. Alternatively, this Gaussian variable can be generated based on other criteria such as SNR or BER [90][91][92]. Since (5-18) exploits the information on the entire support of the pdf, mutual information provides a more robust approximation as shown in [92]. However, if $f(l \mid x= \pm 1)$ is very close to Gaussian, employing different criteria results in similar performance predictions.

In our case, since the output of the equalizer has Gaussian-like distribution and we can directly compute its mean and variance, the output pdf is fully described. Therefore it 
is not necessary to estimate the pdf by simulation. This makes our method considerably simpler than that of [55].

The EXIT charts produced in [55] are generated using large block lengths. In other words, they are useful to predict the asymptotic performance of turbo equalization. In our method, for the decoder we use the BER curve which is empirically generated for the block length of interest. This makes the performance prediction more accurate than that of [55]. Figure 5-8 compares the predicted performance using the proposed method and EXIT chart of [55] with the actual simulation results after four iterations with the mentioned parameters in section 5.2. As can be seen, for low bit error rates, the EXIT chart method prediction is approximately $2.5 \mathrm{~dB}$ away from the actual performance. This value is less than $0.1 \mathrm{~dB}$ by using the proposed BERT chart.

\subsection{Conclusion}

In this chapter we proposed a new BERT chart method for performance analysis of TFDE systems. We showed that the results of this method are consistent with simulation results for BPSK and 16-QAM in adaptive and non-adaptive TFDE. We also showed that this method outperforms the EXIT chart and the previously proposed BERT chart for turbo time domain equalization when block lengths are small (e.g., $10^{3}$ ). 


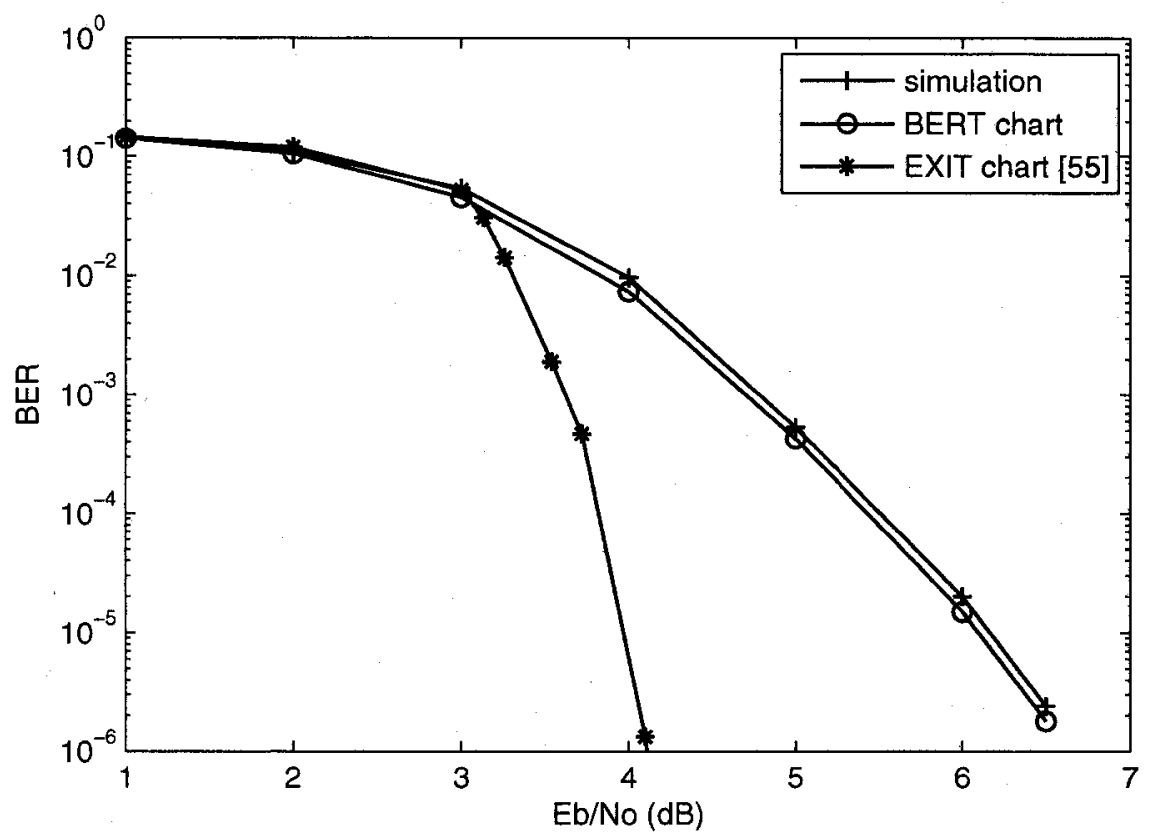

Figure 5-8. Comparison between the BERT chart and EXIT chart for a short block length, BPSK, non-adaptive TFDE. 


\section{Chapter 6 Joint Turbo Frequency Domain Equalization and Synchronization}

\subsection{Introduction}

Carrier frequency synchronization is a known challenge in communication systems. Any deviation from the original frequency in the transmitter or receiver local oscillators, can generate performance degradation. This deviation can have either a deterministic nature like frequency offset or a random nature like phase noise [35][38]. In both cases a time-varying error is present in the phase of the received signal. Block-wise transmission 
methods such as OFDM and SM can tolerate only a fraction of the reciprocal of the total block length as the maximum frequency offset. Thus the performance degradation increases with the block length. This problem has been the subject of many papers especially for multi-carrier transmission, where it can destroy the sub-carriers' orthogonality. Different methods have been suggested for carrier synchronization in multi-carrier systems [98][38][39]. Some of them are based on preamble transmission and some others are based on blind synchronization methods.

In this chapter, we propose a carrier frequency synchronization method for turbo frequency domain equalization. The receiver jointly compensates for the phase error and equalizes the received signal. The idea of using generated information in the previous iterations for synchronization has also been used in [57] and [58]. What distinguishes this work from [58] is that here we consider frequency offset and phase noise estimation, while [58] deals with timing recovery. Besides, the system in [58] is a time domain turbo equalizer while here it is TFDE. In [57] iterative carrier synchronization is performed for an Iterative Block Decision Feedback Equalization (IBDFE) system, while in this thesis the system is TFDE where unlike IBDFE, the decoder is a part of the iterative loop. In [57] only frequency offset is estimated for the IBDFE, while here we consider both frequency offset and PHN for TFDE. For frequency offset, by considering the soft symbols generated from the log likelihood ratios of the decoder output at the previous iteration as known training symbols, the phase error is estimated. The estimation is based on the Maximum Likelihood method and another method similar to what was suggested in [57] for IBDFE. We also find the MAP solution in the presence of both frequency 
offset and phase noise which results in the form of a decision directed Phase Lock Loop (PLL [97]) using LLRs of decoder output.

\subsection{Frequency offset and Phase Noise}

In an ideal system, the transmitter and receiver local oscillators have exactly the same frequency. Yet in reality, there is a time-varying phase difference between these oscillators. The time-varying phase shift can degrade the performance of single carrier or SM systems, however in SM it is less destructive than in multi-carrier systems where the time-varying phase shift can generate inter-data symbol interference. The time-varying phase shift might be caused by either a frequency offset between the oscillators or the phase noise effect. If there is a frequency offset of $\delta f$ between the transmitter and receiver local oscillators, the phase error in the received signal would be $\varphi(t)=2 \pi t \delta f$, or

in the sampled domain $\phi_{n}=\frac{2 \pi n T}{N} \delta f$, where $N$ and $T$ are block size and block duration respectively.

In most systems in addition to the existence of a fixed shift in the oscillation frequency, the PSD might be splattered around this frequency. This effect is known as phase noise in communication systems. In literature, PHN is either defined by the PSD found by measurement [99] or modeled by a continuous Brownian motion or Wiener process where the added phase to the $n$-th symbol is related to the previous one as [35][37]: 


$$
\phi_{n}=\phi_{n-1}+\varepsilon_{n},
$$

where $\varepsilon_{n}$ is a Gaussian distributed random variable with zero mean and variance of $\gamma^{2}=\frac{2 \pi \beta T}{N}$ and $\beta$ is the $3 \mathrm{~dB}$ frequency of the Lorentzian PSD function. Thus the phase added to the $n$-th symbol is Gaussian with zero mean and a variance increasing linearly with time, and the phases are correlated. Most systems suffer from both frequency offset and PHN. In this case the phase error vector would be:

$$
\boldsymbol{\varphi}=\left[\begin{array}{l}
\phi_{1} \\
\phi_{2} \\
\vdots \\
\vdots \\
\vdots \\
\phi_{N}
\end{array}\right]=\left[\begin{array}{l}
2 \pi \delta f T / N \\
4 \pi \delta f T / N \\
\vdots \\
\vdots \\
\vdots \\
2 N \pi \delta T / N
\end{array}\right]+\left[\begin{array}{cccccc}
1 & 0 & 0 & 0 & \ldots \ldots . . & 0 \\
1 & 1 & 0 & 0 & \ldots \ldots . . . & 0 \\
1 & 1 & 1 & 0 & \ldots \ldots \ldots & 0 \\
& & \ddots & & \\
& & & \ddots & & \\
1 & 1 & 1 & 1 & \ldots \ldots . . .1 & 1
\end{array}\right]\left[\begin{array}{c}
\varepsilon_{1} \\
\varepsilon_{2} \\
\varepsilon_{3} \\
\vdots \\
\vdots \\
\varepsilon_{N}
\end{array}\right]
$$

We have associated phase noise and frequency offset with each data symbol $x_{n}$. After passage through a frequency selective channel with sampled impulse response $\left\{h_{n}\right\}$, the received samples are:

$$
r_{n}=\sum_{m=0}^{N_{h}-1} h_{m} x_{n-m} e^{j\left((n-m) \theta+\sum_{k=1}^{n-m} \varepsilon_{k}\right)}+w_{n}^{\prime},
$$

where $N_{h}$ is the length of the channel impulse response, $w_{n}^{\prime}$ is the channel noise, $x_{n}$ is the $n$-th transmitted symbol and $\theta=\frac{2 \pi \delta f T}{N}$.

We make the reasonable assumption that the phase noise bandwidth is much less than the transmission bandwidth, so that $\sum_{k=1}^{n-m} \varepsilon_{k} \approx \sum_{k=1}^{n} \varepsilon_{k}$ for $m<N_{h}$. Then the received sample can be expressed as: 


$$
r_{n}=\left[\sum_{m=0}^{N_{h}-1} h_{m}^{\prime} x_{n-m}\right] e^{j\left(n \theta+\sum_{k=1}^{n} \varepsilon_{k}\right)}+w_{n}^{\prime},
$$

where $h_{m}^{\prime}=h_{m} e^{-j m \theta}$ is the effective channel response to be equalized.

In other words, whether the frequency offset and phase noise are associated with data symbols or with received samples is immaterial, as long as the phase noise process is nearly constant during the channel impulse response duration.

Thus the sampled received signal in an AWGN channel would be

$$
r_{n}=x_{n} e^{j \phi_{n}}+w_{n}^{\prime}=x_{n} e^{j\left(n \theta+\sum_{k=1}^{n} \varepsilon_{k}\right)}+w_{n}^{\prime}
$$

\subsection{Joint Synchronization and Equalization}

Different methods have been proposed for carrier synchronization. Traditionally, synchronization is prior to and disjoint from other receiver modules. These methods can be categorized as preamble-based and blind synchronization methods. In the first category, a known training sequence is transmitted to the receiver and the synchronization algorithm estimates the phase error and compensates for it. In the latter case, known as blind synchronization, no training sequence is transmitted. Here we suggest a joint synchronization and turbo equalization method where the signal is iteratively equalized, and during the same iterations, the phase error is estimated and compensated. At the beginning of each block, the phase error is assumed to be zero which is achieved by the aid of the training sequence. This method can achieve good performance by using the soft symbols generated by the $\tanh ($.$) function, as the known$ 
training sequence to synchronize the system. In the following sub-sections 6.3.1.1 and 6.3.1.2, we first consider a system suffering only from frequency offset. Then in section 6.3.2 we turn our attention to the case where there are both frequency offset and phase noise.

\subsubsection{Frequency offset estimation}

\subsubsection{Synchronization method based on approximate ML criterion (method I)}

The ML criterion is known as one of the best criteria for estimation. For instance, the ML criterion has been applied in [100] to estimate the uniform or Gaussian distributed phase for phase jitter. In [38] a ML-estimator is proposed based on the transmission of a repeated OFDM symbol where the ML decision for the frequency offset estimation is made according to the given information about the first received symbol. In [98] a similar idea has been proposed for a training symbol with two identical halves. In [40] a MLestimator based on the information of the cyclic prefix and its copy, has been suggested.

The ML estimator maximizes the joint conditional density function $f\left(r_{1}, \ldots, r_{N} \mid \theta, x_{1}, \ldots, x_{N}\right)$, which we represent as $f(\mathbf{r} \mid \theta, \mathbf{x})$, with respect to $\theta$ and $\theta=\frac{2 \pi \delta T}{N}$. Since the noise is assumed to be an uncorrelated Gaussian random process, the cost function $J_{1}$ that should be minimized is as follows: 


$$
J_{1}=\sum_{n=1}^{N}\left|r_{n}-x_{n} e^{j n \theta}\right|^{2}
$$

For BPSK or 4-QAM data symbols, $\left|x_{n}\right|^{2}$ is constant. So minimizing $J_{1}$ is equivalent to minimizing $J$, where:

$$
J=-\sum_{n=1}^{N} \operatorname{Re}\left(r_{n}^{*} x_{n} e^{j n \theta}\right)
$$

The phase $\theta_{0}$ which minimizes $J,\left(\left.\frac{\partial J}{\partial \theta}\right|_{\theta=\theta_{0}}=0\right)$, satisfies the following equation:

$$
\sum_{n=1}^{N} n \operatorname{Re}\left(r_{n} x_{n}^{*}\right) \sin \left(n \theta_{0}\right)=\sum_{n=1}^{N} n \operatorname{Im}\left(r_{n} x_{n}^{*}\right) \cos \left(n \theta_{0}\right)
$$

Since $n \theta_{0}$ is assumed to have small value over the block, $\sin ($.$) and \cos ($.$) can be$ estimated by the first terms of the Taylor series. $\sin \left(n \theta_{0}\right) \approx n \theta_{0}, \cos \left(n \theta_{0}\right) \approx 1-\frac{\left(n \theta_{0}\right)^{2}}{2}$. The desired $\theta_{0}$ satisfies the following second order equation:

$$
\theta_{0}^{2} \sum_{n=1}^{N} .5 n^{3} \operatorname{Im}\left(r_{n} x_{n}^{*}\right)+\theta_{0} \sum_{n=1}^{N} n^{2} \operatorname{Re}\left(r_{n} x_{n}^{*}\right)-\sum_{n=1}^{N} n \operatorname{Im}\left(r_{n} x_{n}^{*}\right)=0
$$

We replace the known sequence $\left(x_{n}\right)$ with the estimated soft symbols generated in the feedback loop $\left(\bar{x}_{n}\right)$. Since during the derivation of (6-4) the channel is assumed to be AWGN, we should replace $r_{n}$ with $z_{n}$ which is the equalizer output. The equalizer output distribution, given the data symbols, can be well approximated as Gaussian [22]. Since the noise after the equalizer is not white, the above equations are approximate. The synchronization parts, added to the original block diagram of TFDE, are depicted by 
dashed lines in Figure 6-1. If in each iteration, the reliability of the decisions made by the decoder increases, the estimated soft symbols continually approach the exact value of the transmitted symbols. Therefore the error in the estimated frequency offset continually decreases.

For uniformly distributed $\theta$ and/or for high SNR, the ML estimation is equivalent to the MAP estimator which maximizes $f(\theta \mid \mathbf{r})$. For large SNR, it is also equivalent to estimating the frequency offset from the expected value of $\theta$ given the information about the received and transmitted data, which is

$$
\hat{\hat{\theta}}=E(\theta \mid \mathbf{x}, \mathbf{r})=\int_{-\infty}^{+\infty} \theta f(\theta \mid \mathbf{x}, \mathbf{r}) d \theta
$$

For large SNR where the variance of the Gaussian distribution is small, this integral can be approximated by the mean of the Gaussian random variable.

$$
\int_{-\infty}^{+\infty} \theta f(\theta \mid \mathbf{x}, \mathbf{r}) d \theta \approx \widetilde{\theta}
$$

where $\widetilde{\theta}$ maximizes $f(\theta \mid \mathbf{x}, \mathbf{r})$ which is equal to $\theta_{0}$ found by the ML criterion.

\subsubsection{Synchronization method based on correlating between two halves of the block (method II)}

The maximum likelihood method discussed in section 6.3.1.1 is a very good method in terms of performance. However, ignoring the solution of the second degree equation, it requires $4 N$ multiplications and $3 N$ additions. Here we investigate another synchronization method which was used in [57] for synchronization in IBDFE. This 


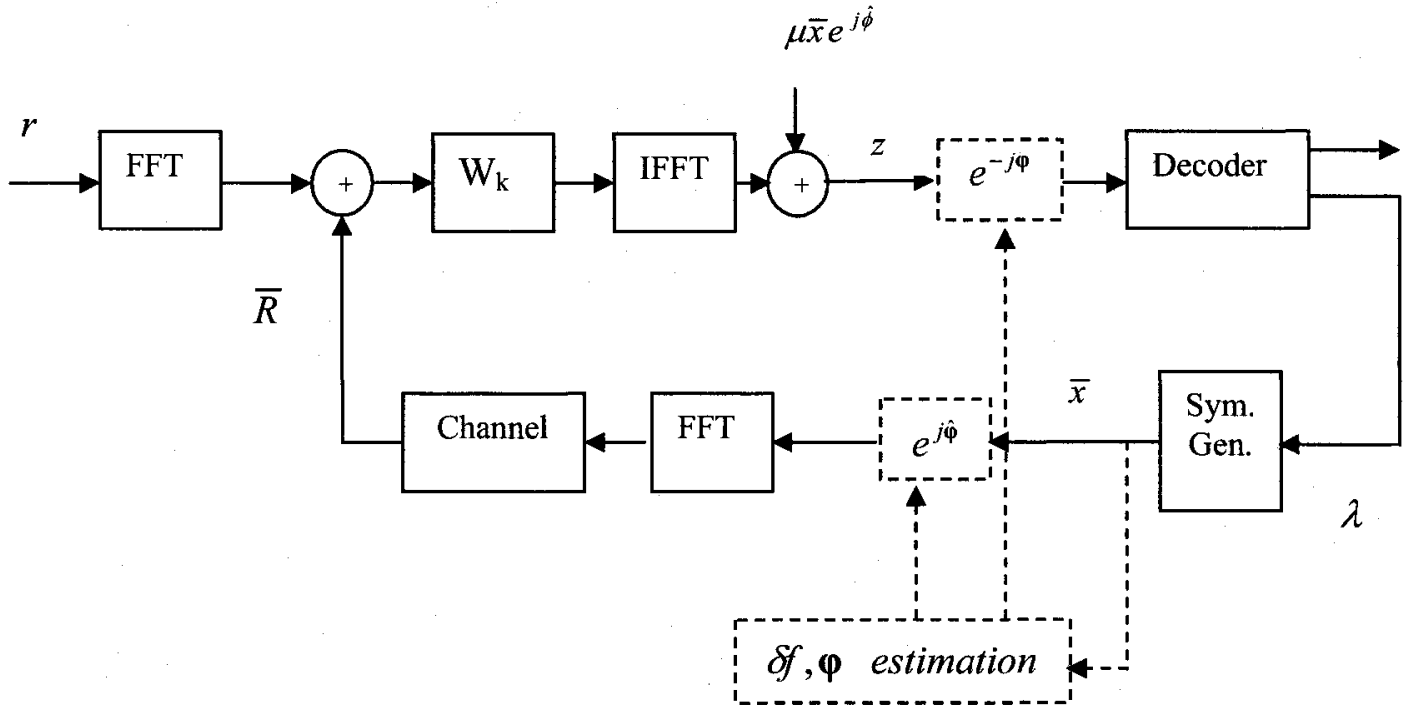

Figure 6-1. Block Diagram of joint turbo equalization and blind synchronization. 
method is less complex than method I. We compare the performance of both methods I and II. If the transmitted symbols are known, for each received data symbol we can define a parameter $m_{k}$ for $k=1, \ldots, N / 2$ as $m_{k}=\frac{\Delta}{x_{k}^{*} x_{k+N / 2}^{*} r_{k+N / 2}}$. In [57] it is shown that $m_{k}$ can be expressed as:

$$
m_{k}=\frac{r_{k}^{*} r_{k+N / 2}}{x_{k}^{*} x_{k+N / 2}} \approx e^{j \frac{N}{2} \theta}+v_{k}^{\prime}
$$

where for large SNR, $m_{1}, \ldots, m_{\frac{N}{2}}$ have equal phases and $v_{k}^{\prime}$ can be approximated as a Gaussian random variable. We can estimate $e^{j \frac{N}{2} \theta}$ by the expected value of $m_{k}$ : $e^{j \frac{N}{2} \theta} \cong E\left(m_{k}\right) \cong \frac{2}{N} \sum_{k=1}^{N / 2} m_{k}$. Thus frequency offset can be estimated as $\hat{\delta f}=\frac{1}{\pi T} \operatorname{Phase}(\eta)$ where $\eta=\frac{\Delta}{N} \sum_{k=1}^{\frac{N}{2}} m_{k}=\frac{2}{N} \sum_{k=1}^{\frac{N}{2}} \frac{r_{k}^{*} r_{k+N / 2}}{x_{k}^{*} x_{k+N / 2}}$.

For large SNR, $\eta$ is the normalized correlation between two halves of the block. Similar to section 6.3.1.1, we can replace $r_{n}$ and $x_{n}$ by $z_{n}$ and $\bar{x}_{n}$ respectively. If the symbols generated in the feedback loop are less reliable and consequently have smaller LLRs, $\bar{x}_{n}$ will be small, and division by small numbers increases the power of $v_{k}^{\prime}$. To prevent this problem, we have two options. We can replace $x_{n}$ by either the Normalized Soft Decisions (NSD) or Hard Decisions (HD). Therefore the metric is calculated as: 


$$
\eta=\frac{2}{N} \sum_{k=1}^{\frac{N}{2}} \frac{z_{k}^{*} z_{k+N / 2}}{\bar{x}_{D, k}^{*} \bar{x}_{D, k+N / 2}}
$$

in which for NSD $\bar{x}_{D, n}=\bar{x}_{N S D, n}=K \frac{\bar{x}_{n}}{\left|\bar{x}_{n}\right|}$ where $K=1$ and $\sqrt{2}$ for BPSK and 4-QAM respectively and $\bar{x}_{D, n}=\bar{x}_{H D, n}=\operatorname{sgn}\left(\operatorname{Re}\left(\bar{x}_{n}\right)\right)+j \operatorname{sgn}\left(\operatorname{Im}\left(\bar{x}_{n}\right)\right)$ for HD. In section 6.4 we compare the performance and Mean Squared Error (MSE) of method II with both metrics. We note that using NSD is possible only when we have equal level constellations like BPSK and 4-QAM.

To compute $\eta$ in (6-10) we need $N / 2$ multiplications and $N / 2$ additions plus $N$ phase rotations for division by $\bar{x}_{D, n}$ (for both NSD and HD). The method of calculating $\bar{x}_{D, n}$ in HD is simpler than that of NSD. Generally method II requires less computational complexity than the ML method.

\subsubsection{Comparison with other synchronization methods}

Since the single carrier systems with frequency domain equalization can be considered as DFT-precoded OFDM, it is important to know whether the synchronization methods for OFDM are applicable for single carrier systems. For this sake, we have selected two efficient synchronization methods for OFDM which do not require transmission of training sequences. The first one, which is proposed in [40] and which we call VSB (Van de Beek et al.) here, uses the information in the cyclic prefix to estimate the frequency offset. The metric calculated in this method is a correlation between the cyclic prefix and 
its copy as: $\xi=\sum_{k=1}^{N_{C P}} r_{k} r_{k+N}^{*}$ where $N_{C P}$ is the cyclic prefix length and the frequency offset is estimated as $\hat{\delta f}=\frac{-1}{2 \pi N}$ phase $(\xi)$. Since the metric $\xi$ is calculated at the beginning of the receiver prior to the equalization process, this method is not iterative.

The complexity of method I, II and VSB are presented in Table 6-1. The order of the complexity in all these methods is proportional to the block length. In particular the least complex one is VSB in which the complexity increases linearly with the cyclic prefix length. The cyclic prefix length is at least equal to the channel impulse response. To achieve a reasonable transmission rate, the block length is roughly ten times larger than the cyclic prefix length. For a comprehensive comparison between these methods, we should also compare the MSE achieved by using these methods and also their linearity range, which are discussed in section 6.4 .

The second synchronization method for OFDM we consider here is the one proposed in [101]. The block diagram of this method is presented in Figure 6-2. The effect of frequency offset in the received OFDM signal after the FFT, is similar to the effect of

Table 6-1 Complexity comparison between the frequency offset compensation methods

\begin{tabular}{|c|c|c|}
\hline & Number of additions & Number of multiplications \\
\hline Method I (per iteration) & $3 \mathrm{~N}$ & $4 \mathrm{~N}$ \\
\hline Method II (per iteration) & $\mathrm{N} / 2$ & $\mathrm{~N} / 2$ \\
\hline Method VSB & $\mathrm{N}_{\mathrm{cp}}$ & $\mathrm{N}_{\mathrm{cp}}$ \\
\hline
\end{tabular}




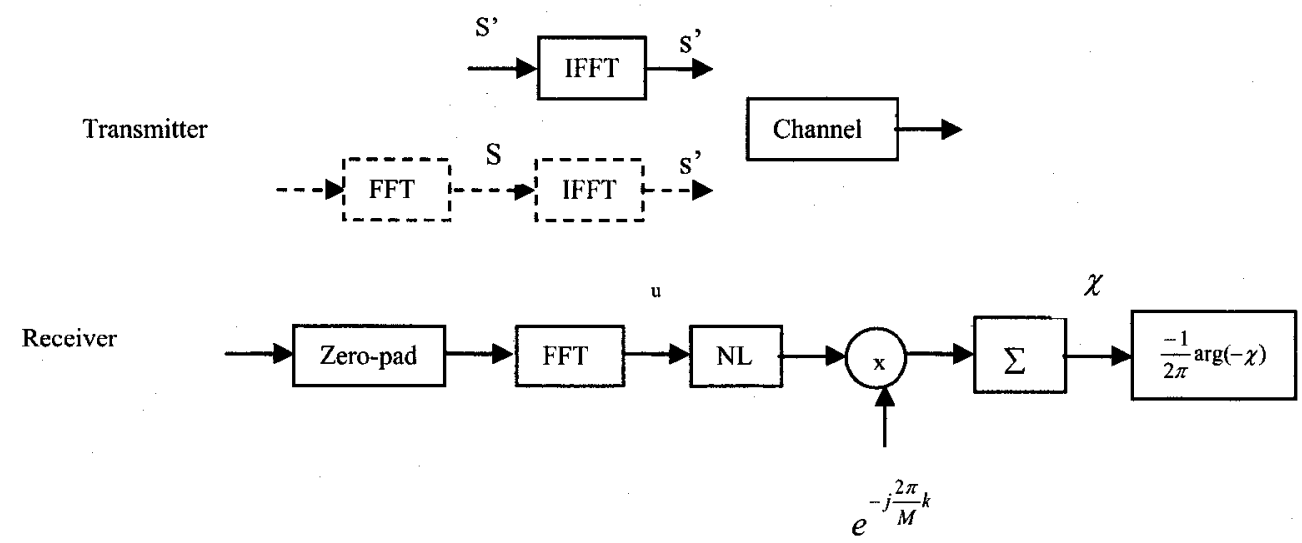

Figure 6-2. Block Diagram of square-law based synchronization for OFDM (SM transmitter is presented by dashed line).

time offset in traditional serial modulation as:

$$
u_{k}=\sum_{m=1}^{N} S_{m}^{\prime} H_{m} f_{1}(k-m-\delta f T)
$$

Therefore an approach similar to the square-law time recovery method proposed in [102] for synchronization of linear modulations can be used. Since the transmitter of single carrier system is as presented in Figure 6-2, there is a similar relationship between $u_{k}$ (the FFT output in the receiver) and $S_{m}^{\prime}$ (the FFT of the transmitted signal) as:

$$
u_{k}=\sum_{m=1}^{N} s_{m}^{\prime} H_{m} f_{1}(k-m-\delta f T)
$$


However we have found that the method of [101] is not applicable to SM because in OFDM the $\left\{S_{m}^{\prime}\right\}$ are from a uniformly distributed constellation with small cardinality, while in SM they are from an approximately Gaussian distributed constellation (due to the law of large numbers).

\subsubsection{Frequency offset and Phase noise estimation based on MAP criterion}

In reality most systems suffer from both frequency offset and phase noise. The added phase in the $n$-th received symbol can be modeled as

$$
\phi_{n}=2 \pi n \frac{\delta f T}{N}+\sum_{k=1}^{n} \varepsilon_{k}=\phi_{n-1}+2 \pi \frac{\delta f T}{N}+\varepsilon_{n}
$$

where the phase noise is assumed a Wiener process and $\varepsilon_{i}$ is Gaussian distributed with zero mean and variance of $\gamma^{2}$. For MAP estimation, the density function $f(\boldsymbol{\varphi} \mid \mathbf{r})$ should be maximized.

$$
f(\boldsymbol{\varphi} \mid \mathbf{r})=\frac{\sum_{\mathbf{x}} f(\mathbf{r} \mid \boldsymbol{\varphi}, \mathbf{x}) p(\mathbf{x}) f(\boldsymbol{\varphi})}{f(\mathbf{r})}
$$

Since $\varepsilon_{n}$ in (6-11) and the channel noise have Gaussian distribution, $f(\mathbf{r} \mid \boldsymbol{\varphi}, \mathbf{x})$ and $f(\boldsymbol{\varphi})$ can be written as:

$$
\begin{gathered}
f(\mathbf{r} \mid \boldsymbol{\varphi}, \mathbf{x})=\frac{1}{\left(2 \pi \sigma^{2}\right)^{N / 2}} \exp \left[-\frac{1}{\sigma^{2}}\left|\mathbf{r}-\mathbf{x} e^{j \varphi}\right|^{2}\right], \\
f(\varphi)=\frac{1}{\left(2 \pi \gamma^{2}\right)^{N / 2}} \exp \left[-\frac{1}{2 \gamma^{2}} \sum_{k=1}^{N}\left(\phi_{k}-\phi_{k-1}-2 \pi \frac{\delta T}{N}\right)^{2}\right], \text { with } \phi_{0}=0 .
\end{gathered}
$$


Therefore

$$
\begin{aligned}
f(\boldsymbol{\varphi} \mid \mathbf{r}) & =\frac{\exp \left[-\frac{|\mathbf{r}|^{2}+|\mathbf{x}|^{2}}{\sigma^{2}}\right]}{f(\mathbf{r})\left[4 \pi^{2} \sigma^{2} \gamma^{2}\right]^{N / 2}} \exp \left[-\frac{1}{2 \gamma^{2}} \sum_{k=1}^{N}\left(\phi_{k}-\phi_{k-1}-2 \pi \frac{\delta f T}{N}\right)^{2}\right] \\
& \times \sum_{\mathbf{x}} p(\mathbf{x}) \exp \left[\frac{2}{\sigma^{2}} \operatorname{Re}\left[\mathbf{r}^{*}{ }^{*} \mathbf{x} e^{j \varphi}\right]\right]
\end{aligned}
$$

$\mathbf{r}^{*} \times \mathbf{x}$ is the scalar multiplication of the vectors $\mathbf{r}$ and $\mathbf{x}$. We can use the a priori information to calculate $p(\mathbf{x})$. For BPSK, the a priori probabilities of each bit found by its LLR in the previous iteration, can be expressed as:

$$
p\left(x_{n}=1 \mid \mathbf{z}\right)=\frac{e^{\lambda_{n}}}{1+e^{\lambda_{n}}}, p\left(x_{n}=-1 \mid \mathbf{z}\right)=\frac{1}{1+e^{\lambda_{n}}} .
$$

Thus we have:

$$
\sum_{\mathbf{x}} p(\mathbf{x}) \cdot \exp \left[\frac{2}{\sigma^{2}} \operatorname{Re}\left[\mathbf{r}^{*} \circ \mathbf{x} e^{j \varphi}\right]\right]=\prod_{k=1}^{N} \cosh \left(\frac{\lambda_{k, 1}}{2}+\frac{2}{\sigma^{2}} \operatorname{Re}\left[r_{k}^{*} e^{j \phi_{k}}\right]\right)
$$

$$
\begin{aligned}
& f(\boldsymbol{\varphi} \mid \mathbf{r})= \\
& \frac{\exp \left[-\frac{|\mathbf{r}|^{2}+|\mathbf{x}|^{2}}{\sigma^{2}}\right]}{f(\mathbf{r})\left[4 \pi^{2} \sigma^{2} \gamma^{2}\right]^{N / 2}} \exp \left[-\frac{1}{2 \gamma^{2}} \sum_{k=1}^{N}\left(\phi_{k}-\phi_{k-1}-2 \pi \frac{\delta f T}{N}\right)^{2}+\sum_{k=1}^{N} \ln \cosh \left(\frac{\lambda_{k, 1}}{2}+\frac{2}{\sigma^{2}} \operatorname{Re}\left[r_{k}^{*} e^{j \phi_{k}}\right]\right)\right]
\end{aligned}
$$

If $x_{i}$ is selected from a BPSK or 4-QAM constellation, $|\mathbf{x}|^{2}$ is constant and maximizing $f(\varphi \mid \mathbf{r})$ is equivalent to minimizing $J$ : 


$$
J=\frac{1}{2 \gamma^{2}} \sum_{k=1}^{N}\left(\phi_{k}-\phi_{k-1}-2 \pi \frac{\delta f T}{N}\right)^{2}-\sum_{k=1}^{N} \ln \cosh \left(\frac{\lambda_{k, 1}}{2}+\frac{2}{\sigma^{2}} \operatorname{Re}\left[r_{k}^{*} e^{j \phi_{k}}\right]\right)
$$

The vector $\varphi$ which minimizes $J$, satisfies the set of equations $\frac{\partial J}{\partial \phi_{i}}=0$ for $i=1, \ldots, N$ which lead to the following recursive equations:

$$
\phi_{k}=2 \phi_{k-1}-\phi_{k-2}+2 \frac{\gamma^{2}}{\sigma^{2}} \operatorname{Im}\left(r_{k-1}^{*} e^{j \phi_{k-1}}\right) \tanh \left(\frac{\lambda_{k-1,1}}{2}+\frac{2}{\sigma^{2}} \operatorname{Re}\left[r_{k-1}^{*} e^{j \phi_{k-1}}\right]\right)
$$

for $k=1, \ldots, N$ and $\phi_{0}=0$. The above equation can be considered as a second order decision directed phase lock loop. Practically, the variance of PHN $\left(\gamma^{2}\right)$ is not known and the above formula has to be modified to:

$\phi_{k}=\phi_{k-1}+\alpha_{1}\left(\phi_{k-1}-\phi_{k-2}\right)+\alpha_{2} \operatorname{Im}\left(r_{k-1}^{*} e^{j \phi_{k-1}}\right) \tanh \left(\frac{\lambda_{k-1,1}}{2}+\frac{2}{\sigma^{2}} \operatorname{Re}\left[r_{k-1}^{*} e^{j \phi_{k-1}}\right]\right)$.

The coefficients $\alpha_{1}$ and $\alpha_{2}$ are not optimized here but are selected such that the system remains stable [97]. When the constellation is 4-QAM, (6-14) changes to:

$$
\begin{aligned}
& \sum_{\mathbf{x}} p(\mathbf{x}) \exp \left[\frac{2}{\sigma^{2}} \operatorname{Re}\left[\mathbf{r}^{*} \cdot \mathbf{x} e^{j \varphi}\right]\right]= \\
& \prod_{k=1}^{N}\left[\cosh \left(\frac{\lambda_{k, 1}}{2}+\frac{\lambda_{k, 2}}{2}+\frac{2}{\sigma^{2}} \operatorname{Re}\left[r_{k}^{*}(1+j) e^{j \phi_{k}}\right]\right)+\cosh \left(\frac{\lambda_{k, 1}}{2}-\frac{\lambda_{k, 2}}{2}+\frac{2}{\sigma^{2}} \operatorname{Re}\left[r_{k}^{*}(1-j) e^{j \phi_{k}}\right]\right),\right.
\end{aligned}
$$

where $\lambda_{k, 1}$ and $\lambda_{k, 2}$ are the LLR of the first and second bit of the $k$-th 4-QAM symbol or correspondingly the LLR of real and imaginary parts of the $k$-th symbol. If we replace 
(6-19) in (6-13) and maximize $f(\varphi \mid \mathbf{r})$ similar to the BPSK case, we find another set of recursive equations for the phases. These equations are as follows (for $k=1, . ., N$ and $\left.\phi_{0}=0\right)$ :

$\phi_{k}=$

$\phi_{k-1}+\alpha_{1}\left(\phi_{k-1}-\phi_{k-2}\right)+\alpha_{2} \frac{\operatorname{Im}\left(r_{k-1}^{*} e^{j\left(\phi_{k-1}+\frac{\pi}{4}\right)}\right) \sinh \left(t_{k-1,1}\right)+\operatorname{Im}\left(r_{k-1}^{*} e^{j\left(\phi_{k-1}-\frac{\pi}{4}\right)}\right) \sinh \left(t_{k-1,2}\right)}{\cosh \left(t_{k-1,1}\right)+\cosh \left(t_{k-1,2}\right)}$

where we define $t_{k, 1}$ and $t_{k, 2}$ as:

$$
\begin{aligned}
& t_{k, 1}=\frac{\lambda_{k, 1}}{2}+\frac{\lambda_{k, 2}}{2}+\frac{2 \sqrt{2}}{\sigma^{2}} \operatorname{Re}\left[r_{k}^{*} e^{j\left(\phi_{k}+\frac{\pi}{4}\right)}\right], \\
& t_{k, 2}=\frac{\lambda_{k, 1}}{2}-\frac{\lambda_{k, 2}}{2}+\frac{2 \sqrt{2}}{\sigma^{2}} \operatorname{Re}\left[r_{k}^{*} e^{j\left(\phi_{k}-\frac{\pi}{4}\right)}\right] .
\end{aligned}
$$

We note that similar to section 6.3.1.1 and 6.3.1.2, in (6-18), (6-20) and (6-21) $r_{k}$ should be replaced by the equalizer output $z_{k}$ which can be well approximated as a Gaussian random variable. The complexity of this method for BPSK and 4-QAM are presented in Table 6-2.

\subsection{Performance of synchronization methods}

We consider a TFDE using a rate one half regular $(3,6)$ LDPC code with block length of $N=1008$. The BP algorithm is used for decoding and $I_{i}=4, I_{o}=4$. The bandwidth is 
$20 M H z$ and the channel is presented in Table 4-1. We consider BPSK and 4-QAM constellations. The corresponding DFT lengths are 1008 and 504 respectively.

The performance degradation resulting from uncompensated frequency offset are presented in Figure 6-3, Figure 6-4 and Figure 6-5. For bit error rates smaller than 0.1, there is at least $1 \mathrm{~dB}$ and $3 \mathrm{~dB}$ performance loss for BPSK when $\delta f T=0.1$ and $\delta f T=0.2$ respectively. This performance loss is at least $2 \mathrm{~dB}$ for $4-\mathrm{QAM}$ when $\delta f T=0.1$, implying that it is more sensitive to carrier frequency instabilities than BPSK. Therefore we only consider $\delta f T=0.1$ for 4-QAM which is a rather large amount for the frequency offset in practice. By using method I and II (NSD and HD), we are able to compensate for these performance degradations as presented in Figure 6-3, Figure 6-4 and Figure 6-5. Both methods I and II are able to improve the performance considerably. For BPSK, when $\delta f T=0.1$ the resulting performance is only $0.05 \mathrm{~dB}$ away from that of the frequency offset-free system. The corresponding value for $\delta f T=0.2$ is $0.15 \mathrm{~dB}$ which is negligible compared to the performance degradation without synchronization.

For 4-QAM, the performance of a system using method $\mathrm{I}$ is only $0.1 \mathrm{~dB}$ and for method II is $0.3 \mathrm{~dB}$ away from the performance of a system without any frequency offset. This

Table 6-2 Complexity of Phase Noise compensation method for BPSK and QPSK

\begin{tabular}{|c|c|c|}
\hline Constellation & $\begin{array}{c}\text { Number of additions } \\
\text { (per iteration) }\end{array}$ & $\begin{array}{c}\text { Number of multiplications } \\
\text { (per iteration) }\end{array}$ \\
\hline BPSK & $4 \mathrm{~N}$ & $5 \mathrm{~N}$ \\
\hline QPSK & $11 \mathrm{~N}$ & $6 \mathrm{~N}$ \\
\hline
\end{tabular}




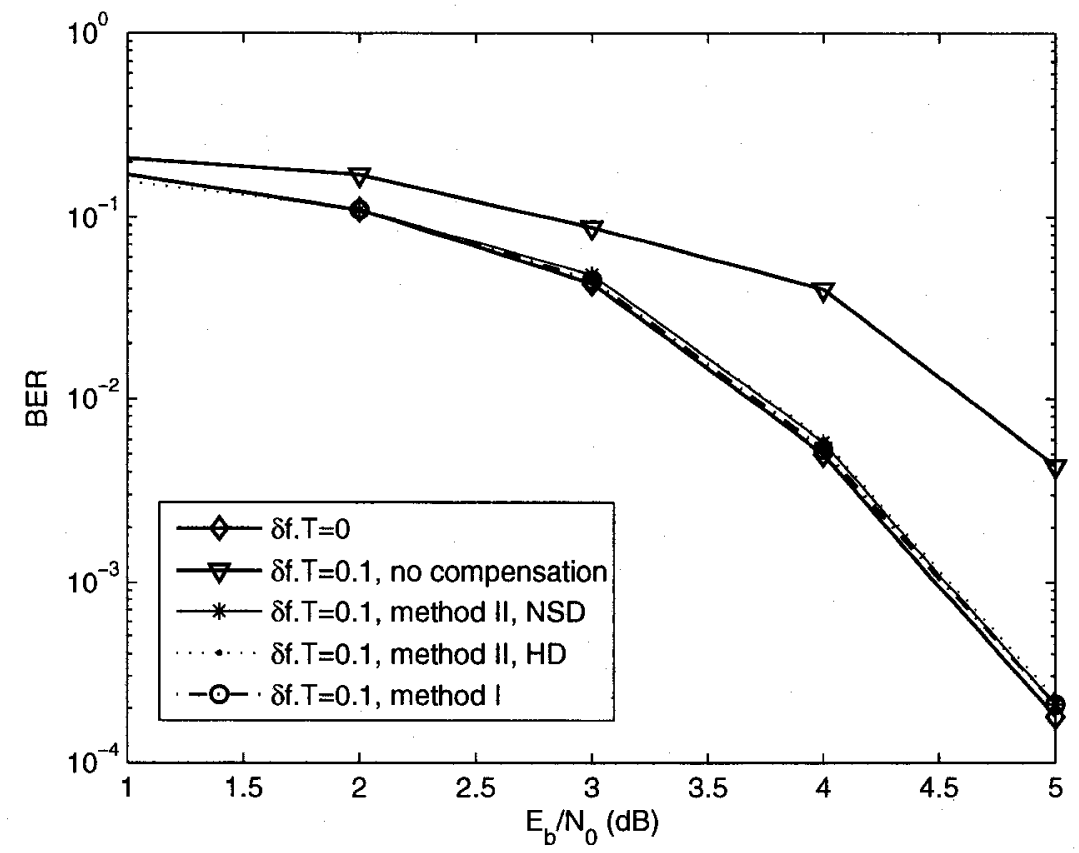

Figure 6-3. Performance of frequency offset compensation methods (I \& II) for BPSK constellation, $\delta f=0.1$.

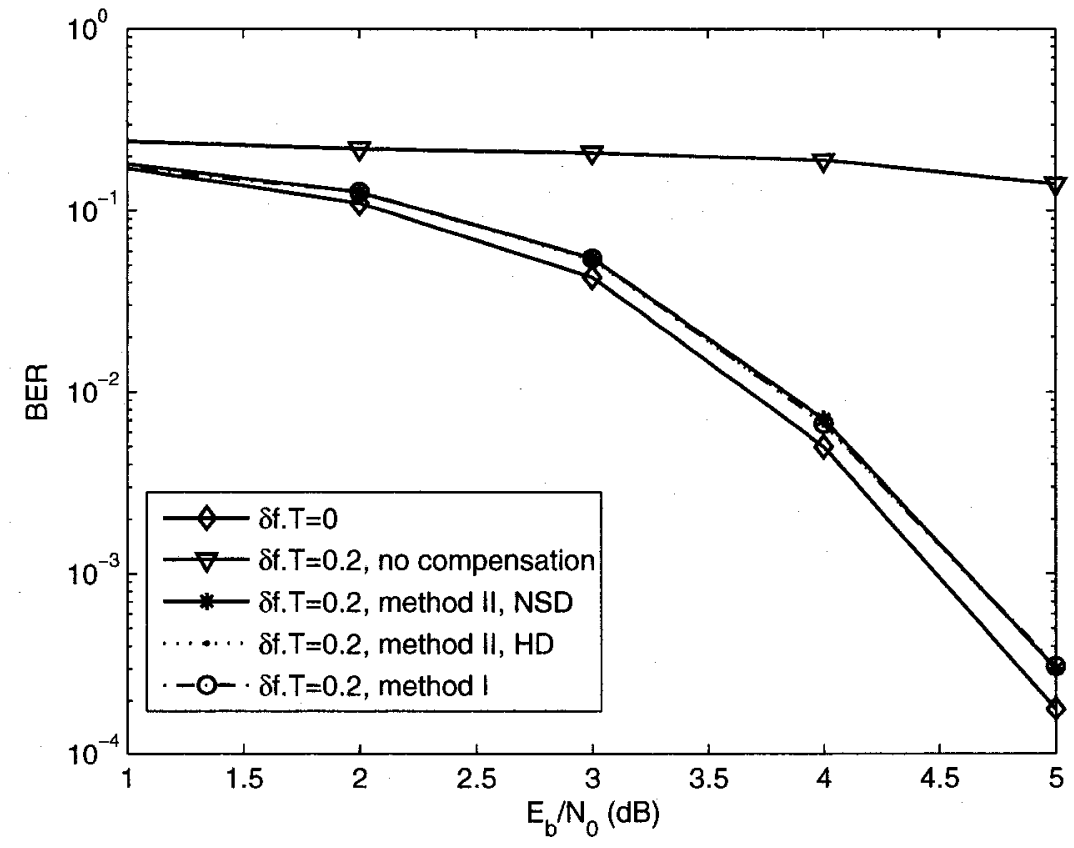

Figure 6-4. Performance of frequency offset compensation methods (I \& II) for BPSK constellation, $\delta f T=0.2$. 


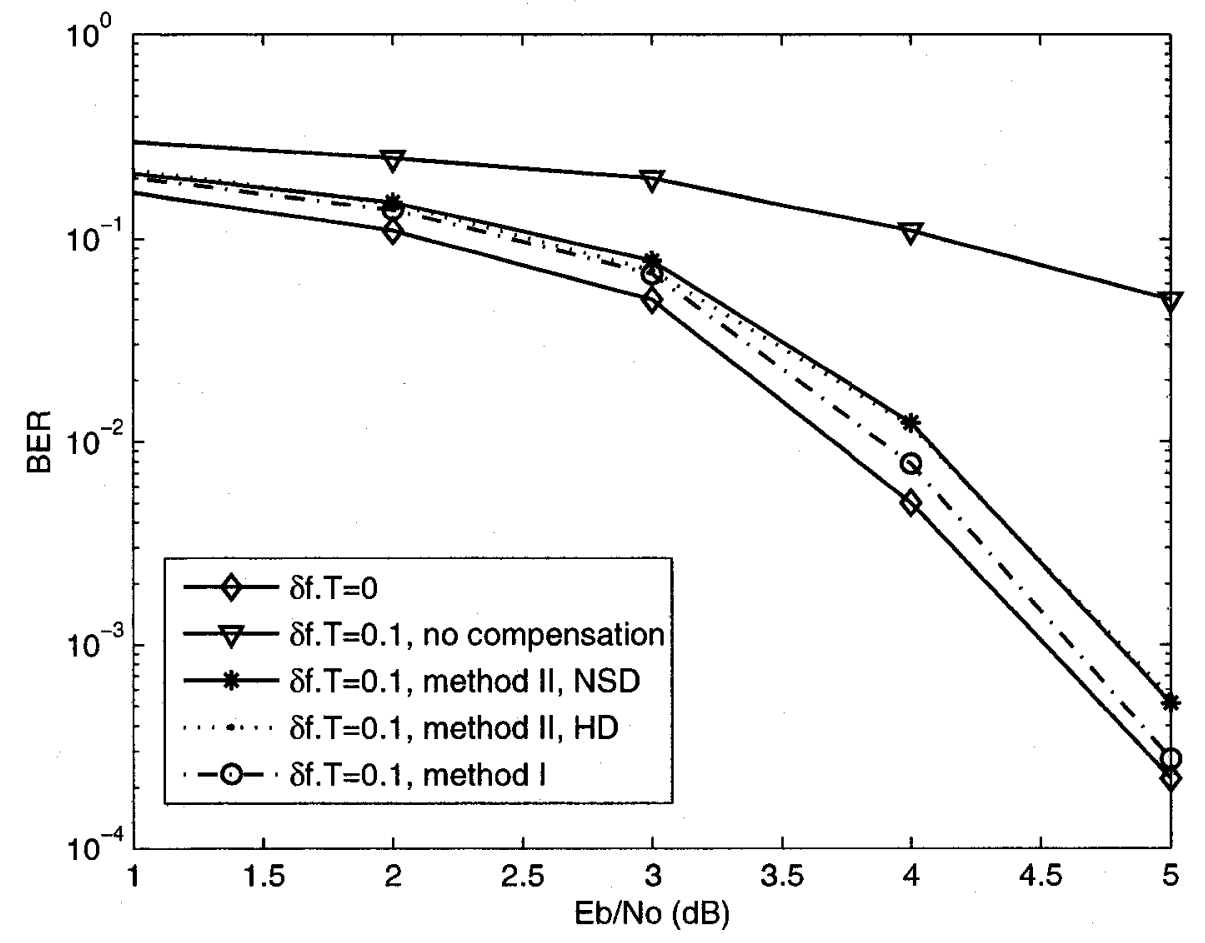

Figure 6-5. Performance of frequency offset compensation methods (I \& II) for 4-QAM constellation, $\delta f T=0.1$. 
shows that method I (the ML method) performs better than method II. As can be seen, the difference between the BER performance when HD and NSD are used in the metric of method II is quite negligible. Considering the fact that HD has less computational complexity than NSD, it is of more interest from practical point of view.

The difference between methods I and II (NSD and HD) is also depicted in Figure 6-6 where the mean squared error of the estimation in each iteration is presented for BPSK and $\delta f T=0.1$. As presented, method II with HD has similar MSE to that of NSD in the first iteration. However in other iterations by using HD in the metric we are able to achieve smaller MSE. Also the MSE of method I is smaller than that of method II (for both HD and NSD). Since all MSEs are small enough after four iterations, the difference between MSEs does not appear in the corresponding BER performance in Figure 6-3. For 4-QAM, the difference between MSEs would be larger. In Figure 6-6 we also compare the MSE achieved by method I and II with that of the VSB method. As presented, the MSE of VSB is larger than that of other methods even for the first iteration. In Figure 6-7 we present the linearity range of these methods. This figure illustrates the average of the corresponding estimated values of normalized frequency offset for a range of the frequency offset. As presented, all these methods in a typical range for the normalized frequency offset, which is up to 0.1 to 0.15 , are linear. The VSB method has a wider linearity range. Since the performance of method I and II depend on the decisions in the feedback loop, in the presence of a large amount of frequency offset (0.3-0.4) the symbols generated in the feedback loop would be less reliable which results in poor performance of the synchronization method. This suggests using a combination of the VSB and method I or II for dealing with large frequency offset. The frequency offset 


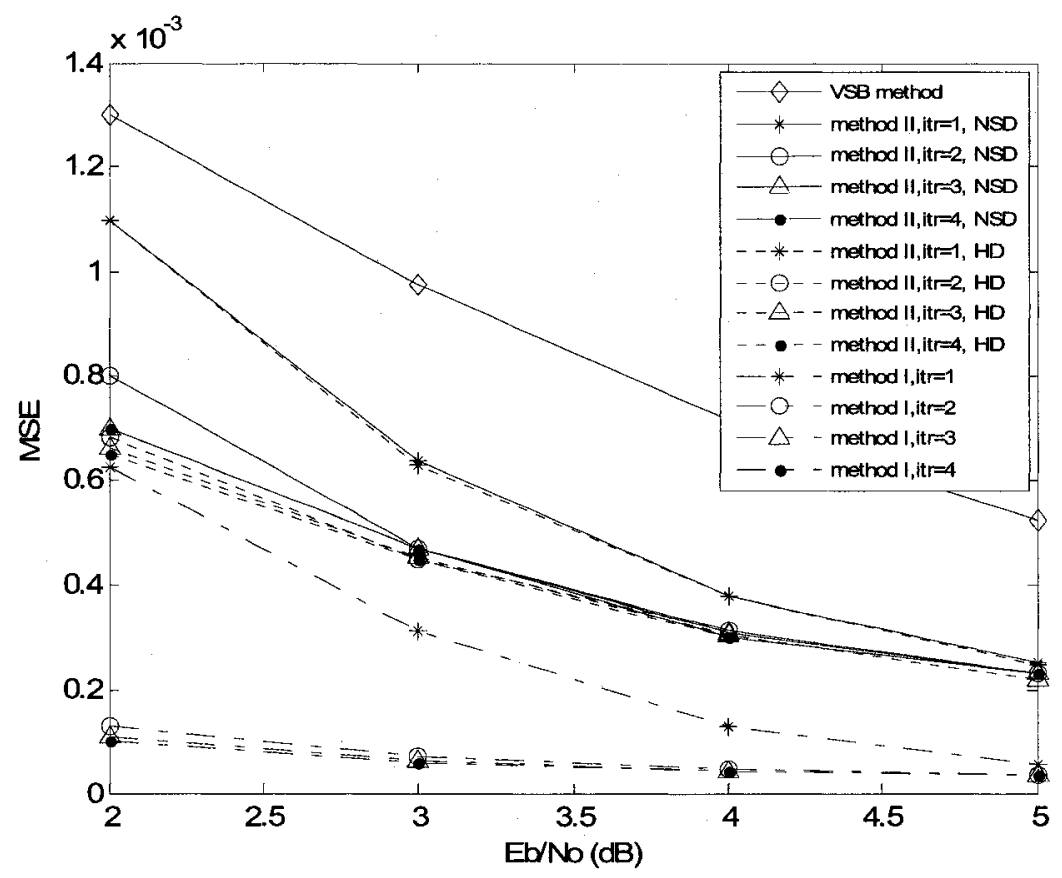

Figure 6-6. MSE of estimation for methods I, II and VSB for BPSK and $\delta f T=0.1$ (itr= iteration number).

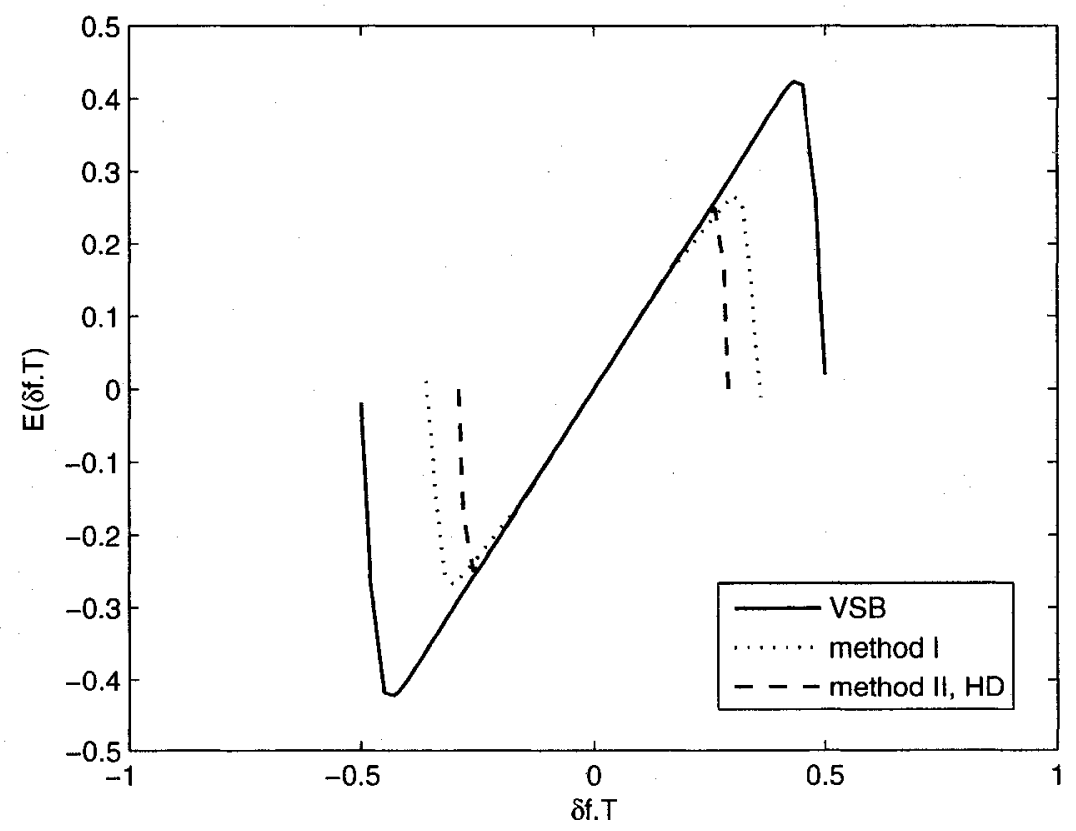

Figure 6-7. Linearity range of method I, II (HD) and VSB. 
is compensated partially in the first iteration by VSB and then by using method I or II the performance of synchronization will be improved during the next iterations.

In the presence of both frequency offset and phase noise, the performance degradation is much larger than the corresponding value in the presence of only frequency offset. As depicted in Figure 6-8, for bit error rates smaller than 0.01 there is at least $2.4 \mathrm{~dB}$ performance loss for BPSK when $\delta f T=0.1$ and $\gamma=0.01$, which is about $1.5 \mathrm{~dB}$ larger than the corresponding value for the case with $\delta f T=0.1$ and $\gamma=0$ presented in Figure 6-3. The difference is much larger for $\gamma=0.03$. Figure 6-8 and Figure 6-9 also show the performance improvement achieved by the decision directed estimation method (labeled as "synchronization" in the figures) discussed in section 6.3.2. As depicted, this method can improve the performance considerably. For example, for BPSK when $\delta f T=0.1$ and $\gamma=0.01$ there is about $2.2 \mathrm{~dB}$ improvement at bit error rate of 0.01 . The improvement is much larger for $\delta f T=0.1$ and $\gamma=0.03$.

In Figure 6-9 we only consider $\delta f T=0.1$ and $\gamma=0.01$ for $4-\mathrm{QAM}$ due to its higher sensitivity to the phase error. In this case, there is also significant performance improvement. For instance there is $2.6 \mathrm{~dB}$ improvement at bit error rate of 0.05 . Both Figure 6-8 and Figure 6-9 improvement becomes even larger for smaller bit error rates. As presented in Appendix $G$ the proposed synchronization method also works efficiently for channel C2 whose model is presented in Table F-1. For this channel we also assumed that the mobile terminal has the velocity of $50 \mathrm{~km} / \mathrm{h}$. 


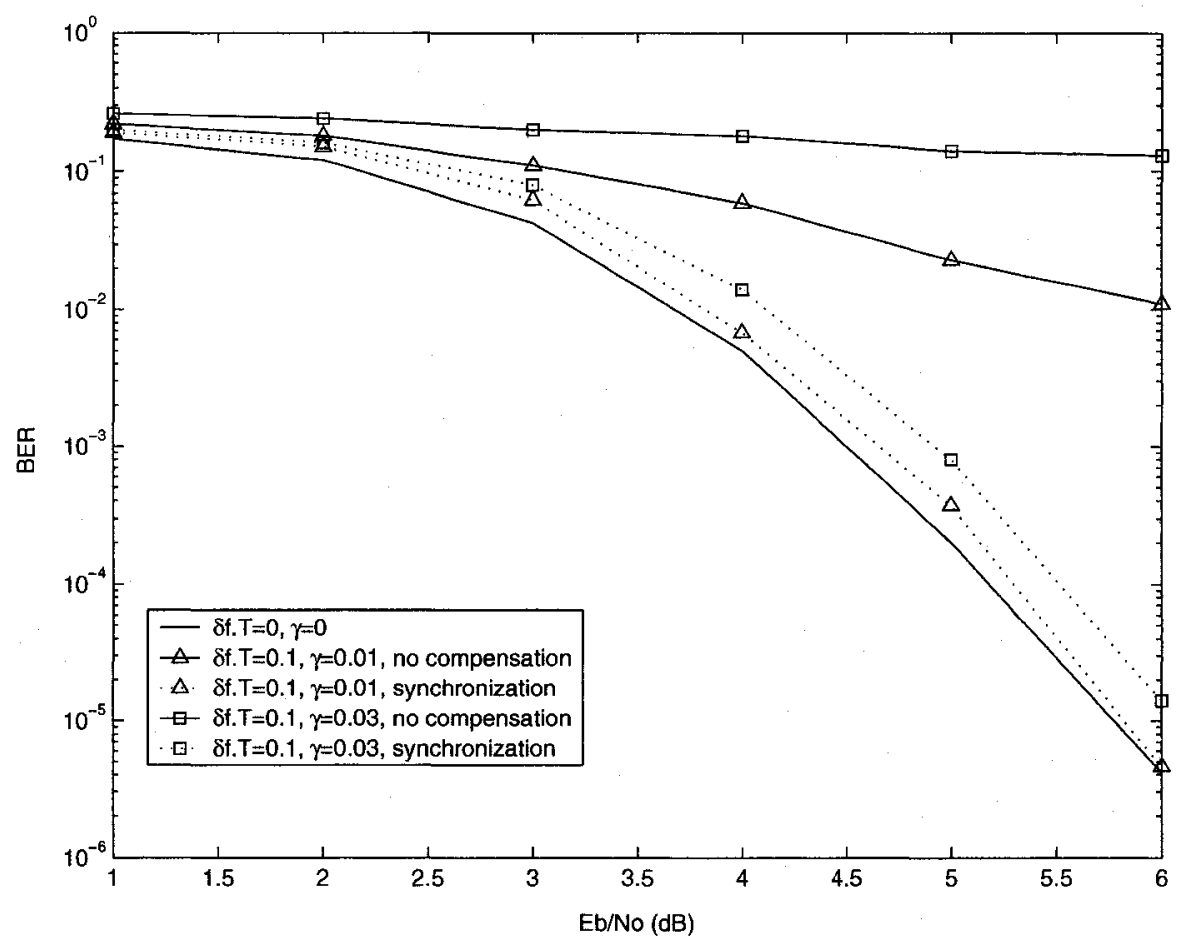

Figure 6-8. Performance of the MAP synchronization method in the presence of frequency offset and PHN for BPSK constellation.

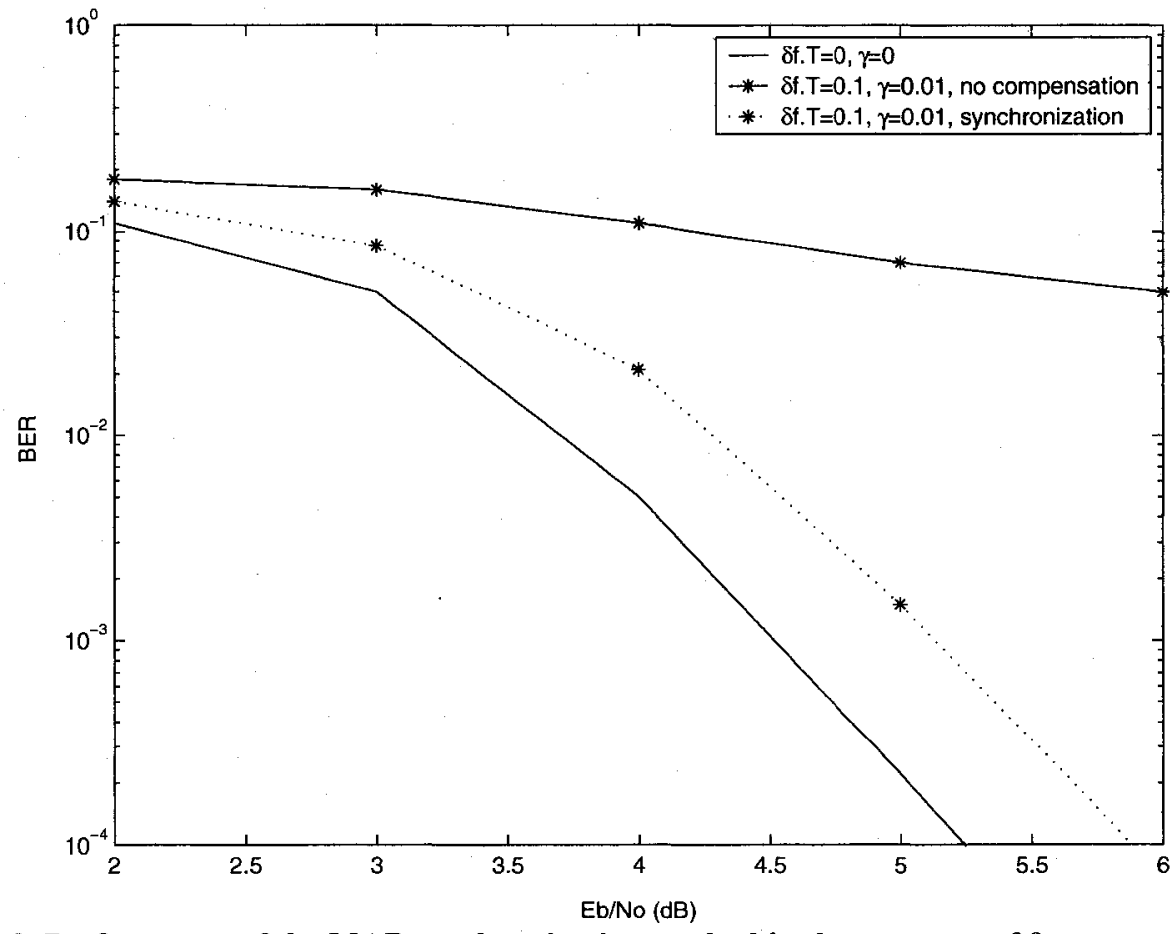

Figure 6-9. Performance of the MAP synchronization method in the presence of frequency offset and PHN for 4-QAM constellation. 


\subsection{Conclusion}

In this chapter we investigated the effect of frequency offset and phase noise on the performance of a frequency domain turbo equalization. We proposed two blind iterative frequency offset compensation methods for turbo equalizers. Both methods can compensate the performance degradation caused by frequency offset for BPSK while the method based on the ML criterion can achieve smaller MSE and better performance for 4-QAM. In these methods, by using the information provided during previous iterations the frequency offset is estimated iteratively. We also investigated a MAP estimator for a more realistic case in which the system suffers from both frequency offset and phase noise. The MAP gave us some recursive formulas which can be considered as a PLL using the LLRs to have decision directed estimations. All these methods require no extra bandwidth for transmission of a training sequence. By using these methods, after a few iterations, the performance can be improved significantly. 


\section{Chapter 7 Concluding Remarks and Future}

\section{Work}

\subsection{Summary of Contributions}

In this dissertation we looked into some physical layer problems for serial modulation systems. In Chapter 3, we investigated the high power amplification dilemma for OFDM and SM signals. The PSD and amplitude distribution of both systems for power amplifiers with different back-off values were compared. We also proposed a modified SLM method for out-of-band radiation reduction of SM signals which significantly decreases the out-of-band components. We also showed that PAPR of SM-CDMA with 
complex spreading is smaller than that of MC-CDMA. In contrast to complex spreading, real spreading increases the PAPR of SM-CDMA even for equal-level constellations. For multi-level constellations this effect is so significant that makes the PAPR of SM-CDMA comparable with that of MC-CDMA.

In Chapter 4, we considered adaptive and non-adaptive TFDE. We examined convolutional and LDPC codes. By performance and complexity comparison between the considered cases, we showed the efficiency of each scheme. This chapter prepared the ground for the discussion in Chapter 5 , where we proposed the BERT chart as a promising tool for the performance evaluation of the turbo frequency domain equalization. We derived the BERT chart for adaptive and non-adaptive TFDE while we considered different constellations. We also showed that this method outperforms the EXIT chart due to using empirically, not asymptotically, derived curves in the chart.

In Chapter 6, we studied the effect of oscillator instability on the performance of a SM system using turbo frequency domain equalization. We suggested two blind joint synchronization and equalization methods based on different criteria for frequency offset. Both methods can achieve the original performance for BPSK. For 4-QAM, the maximum likelihood method can compensate for the effect of frequency offset perfectly. We also proposed a synchronization method (which can be regarded as a second order decision directed PLL) in the presence of both frequency offset and phase noise. This method can significantly compensate for the phase error effect as the performance loss would be about $0.3 \mathrm{~dB}$ and $0.5 \mathrm{~dB}$ for BPSK and 4-QAM constellations respectively. 


\subsection{Future work}

In this section we review some possible future research to pursue the work which has been performed in this thesis.

\subsubsection{Iterative Demapping TFDE}

TFDE is a promising method due to the capability of achieving good performance by the iterative structure and simplicity of implementation by frequency domain equalization. To respond to the demand for spectrally efficient transmission methods, taking higher order constellations into service is desirable.

For higher order constellations, we can effectively improve the performance if we take the bits' a priori information into account in the demapping. Therefore we have a feedback from the decoder to the demapper. This scheme is called iterative demapping (ID).

To show how using this information enables us to improve the performance, we consider two extreme cases when there is no a priori information available and when we have perfect a priori information. To minimize the error probability in the absence of a priori information for a fixed set of constellation points, we should assign the symbols to the constellation points such that the Euclidean distance between the symbols whose Hamming distance is one would be minimized. In this case the Gray mapping is the most efficient mapping. In contrast with this case, when we decide about a specific bit in the presence of perfect a priori information about the other bits, the Euclidean distance between the constellation points whose symbols have the Hamming distance of one should be maximized. Therefore in the presence of a priori information, other mapping 
schemes like MSP (Modified Set Partitioning) and Anti-Gray perform better than Gray mapping. Figure 7-1 and Figure 7-2 present Gray and MSP mapping. For each mapping the distance between two symbols with Hamming distance of one, which differ only in the third bit, are presented. As can be seen in this case if we know the value of the first, second and forth bits, the decision error for MSP mapping is smaller than that of Gray mapping.

The block diagram of ID-TFDE is presented in Figure 7-3. In the feed-forward path after equalization, the demapper calculates the LLR of each bit using the feedback information. The output LLR of demapper for the $k$-th bit of the $n$-th symbol is:

$$
\lambda_{n, k}^{\prime}=\ln \frac{P\left(b_{n, k}=0\right)}{P\left(b_{n, k}=1\right)}=\ln \frac{\sum_{s \in S_{k, 0}} e^{-\frac{\left|z_{n}-s\right|^{2}}{\sigma^{2}}} P(s)}{\sum_{s \in S_{k, 1}} e^{-\frac{\left|z_{n}-s\right|^{2}}{\sigma^{2}}} P(s)},
$$

where $S_{k, 0}$ and $S_{k, 1}$ denote the subset of the constellation points in which the $k$-th bit is 0 or 1 respectively. In the first iteration, where there is no a priori information available, all symbols in $S_{k, b}$ are equiprobable thereby $P(s)=\frac{1}{8}$. In other iterations, $P(s)$ is determined based on the probabilities of other bits in the symbol. If the reliability of the feedback information increases gradually as iterations continue, the performance of both interference cancellation and demapping will be improved.

In ID-TFDE similar to the original TFDE we should calculate the soft symbols. For mappings other than Gray presented in Figure 7-1, the formula for soft symbol is different from the one in (4-8). By a similar method as the one in Chapter 4, soft symbols for MSP and Anti-Gray are calculated based on (7-2) and (7-3) respectively. 


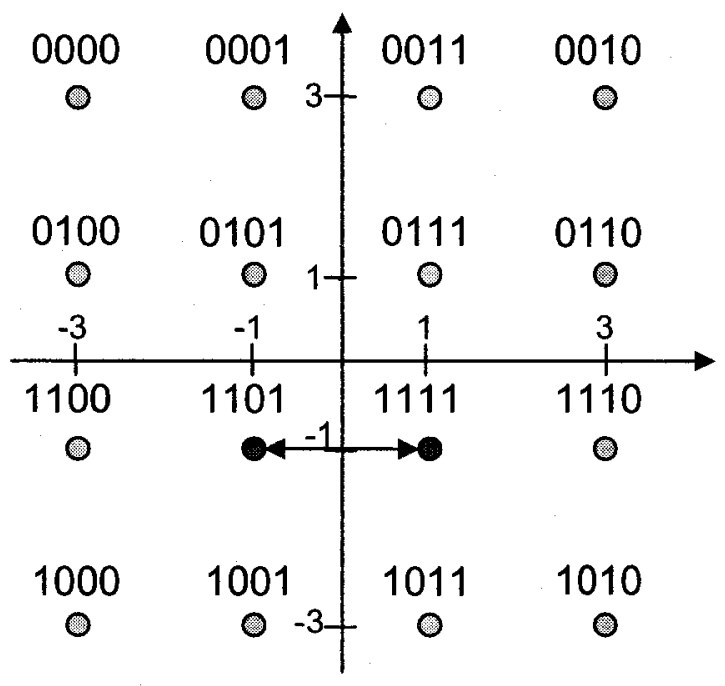

Figure 7-1. Gray mapping for 16-QAM.

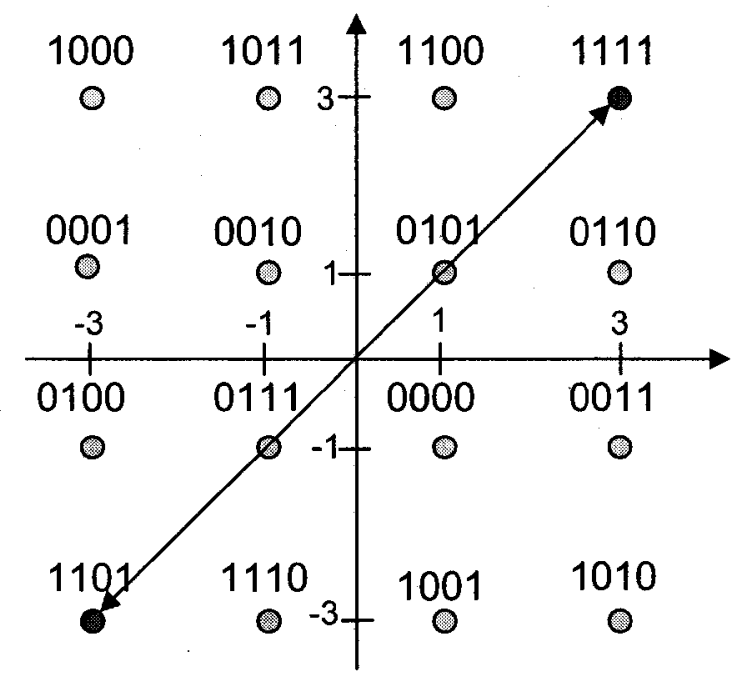

Figure 7-2. MSP mapping for 16-QAM. 


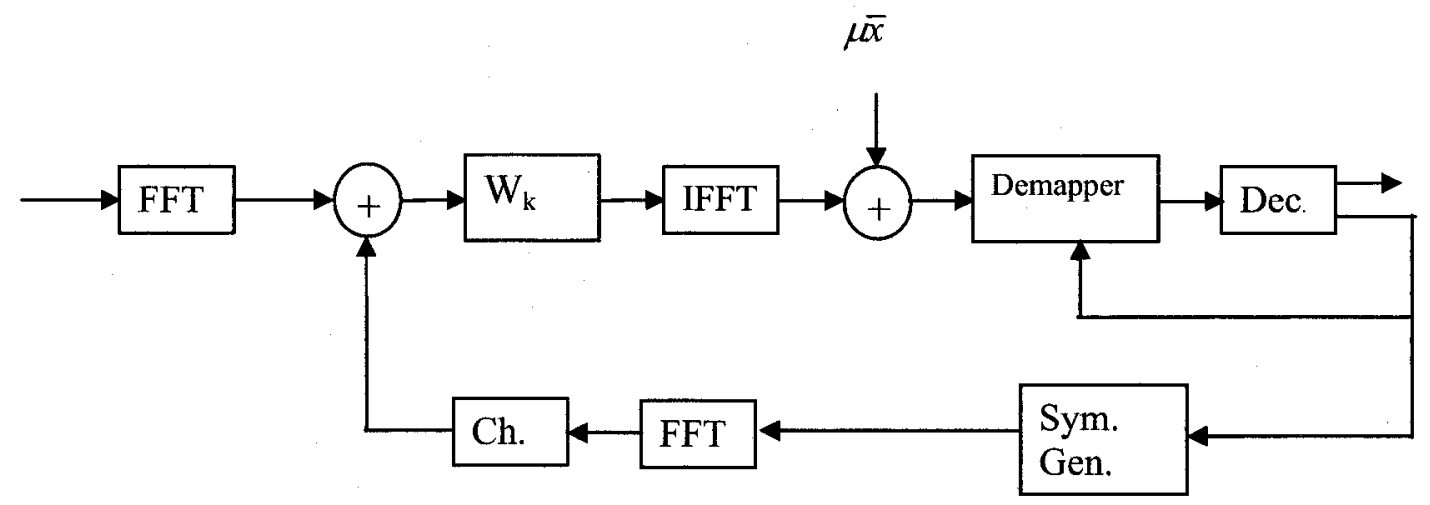

Figure 7-3. Block diagram of ID-TFDE.

$$
\begin{aligned}
\bar{x}_{n}= & -\tanh \left(\frac{\lambda_{n, 3}}{2}\right)\left(1-2 \tanh \left(\frac{\lambda_{n, 1}}{2}\right) \tanh \left(\frac{\lambda_{n, 2}}{2}\right) \tanh \left(\frac{\lambda_{n, 4}}{2}\right)\right) \\
& +j \tanh \left(\frac{\lambda_{n, 3}}{2}\right) \tanh \left(\frac{\lambda_{n, 4}}{2}\right)\left(1-2 \tanh \left(\frac{\lambda_{n, 1}}{2}\right)\right), \\
\bar{x}_{n}= & -\tanh \left(\frac{\lambda_{n, 3}}{2}\right)\left(2 \tanh \left(\frac{\lambda_{n, 4}}{2}\right)+1\right)-j \tanh \left(\frac{\lambda_{n, 1}}{2}\right)\left(2 \tanh \left(\frac{\lambda_{n, 2}}{2}\right)+1\right) .
\end{aligned}
$$

The performance of ID-TFDE with these mappings is presented in Figure 7-4. The parameters are the same as in Chapters 5.

\subsubsection{BERT chart analysis of ID-TFDE}

An interesting problem in the context of ID-TFDE which can be considered in the continuation of this work, is analyzing the performance of ID-TFDE by BERT chart. The BERT chart of ID-TFDE would be a three dimensional chart whose axes are

$$
\begin{aligned}
& \text { 1- } P_{E q, o u t}, P_{D e m, i n, 1}, \\
& \text { 2- } P_{D e c, o u t}, P_{D e m, i n, 2}, P_{E q, i n}, \\
& \text { 3- } P_{D e m, o u t}, P_{D e c, \text { in }} .
\end{aligned}
$$




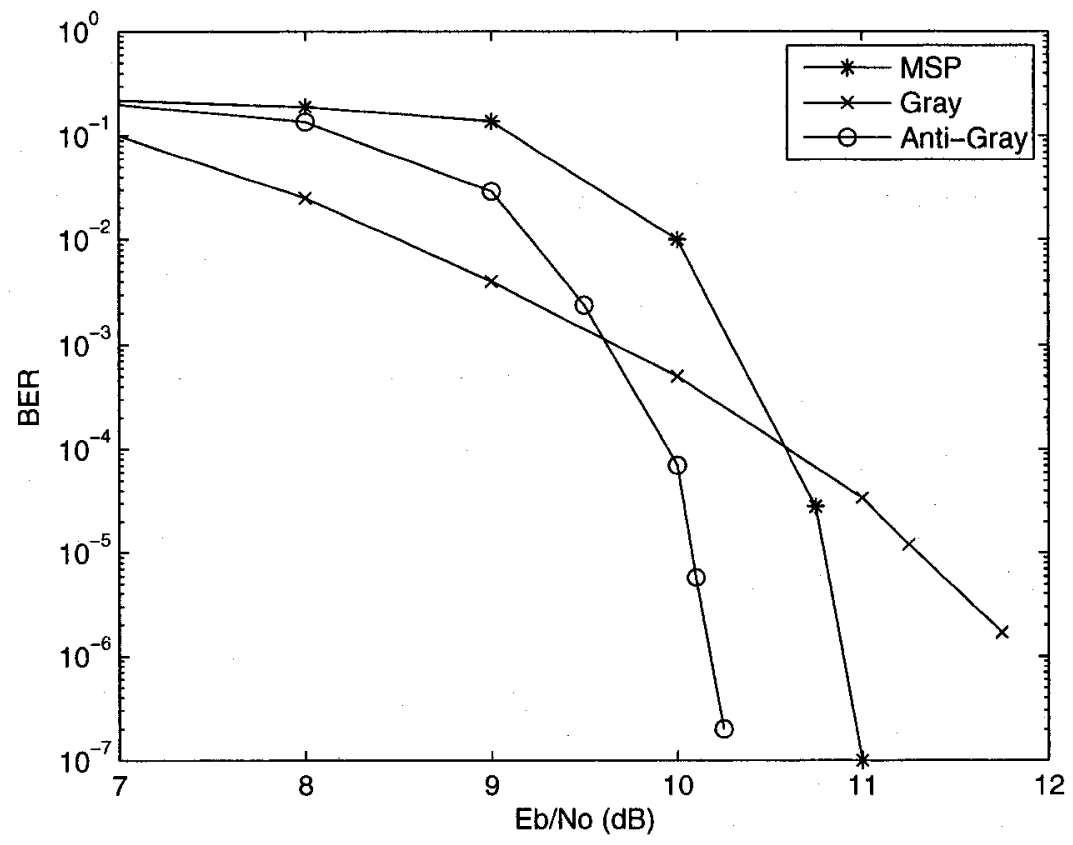

Figure 7-4. Performance of ID-TFDE with Gray, MSP and Anti-Gray mapping, 16-QAM

constellation.

The demapper BER which is a two-input function $\left(P_{D e m, \text { out }}=\mathfrak{J}\left(P_{E q, \text { out }}, P_{D e c, \text { out }}\right)\right)$ will be a surface in the chart. This function for each mapping can be generated once. Another difference between the new BERT chart and the one for TFDE is that $E^{i}\left(\bar{x}_{n} \bar{x}_{n}^{*}\right), E^{i}\left(x_{n} \bar{x}_{n}^{*}\right)$ and $E^{i}\left(x_{n}^{*} \bar{x}_{n}\right)$ for mappings other than Gray, differ from those of Gray mapping. If we use (7-2) for soft estimated symbol, by a similar method to the one presented in Appendix E we can derive the equation for MSP mapping as

$$
E^{i}\left(\bar{x}_{n} \bar{x}_{n}^{*}\right)=E^{i}\left(x_{n} \bar{x}_{n}^{*}\right)=E^{i}\left(x_{n}^{*} \bar{x}_{n}\right)=\Delta_{i}+\Delta_{i}^{2}+4 \Delta_{i}^{3}+4 \Delta_{i}^{4},
$$

The variance of the equalizer output would be: 


$$
\Sigma_{i}^{2}=\sigma^{2} \zeta+\left(\rho-|\mu|^{2}\right)\left(10-\Delta_{i-1}-\Delta_{i-1}^{2}-4 \Delta_{i-1}^{3}-4 \Delta_{i-1}^{4}\right) .
$$

For BERT chart analysis of ID-TFDE these steps should be followed:

(1) Compute $\mu$ and $\Sigma_{0}^{2}$ from (4-14) and (5-3).

(2) Set $P_{\text {Dec,out }}=0.5$.

(3) Compute the equalizer output $\operatorname{BER}\left(P_{E q, o u t}\right)$.

(4) From Demapper function $\mathfrak{I}$ find the corresponding demapper output BER $\left(P_{\text {Dem,out }}=\mathfrak{I}\left(P_{E q, \text { out }}, P_{\text {Dec,out }}\right)\right)$.

(5) From the decoder $\mathrm{BER}_{\text {in }}-\mathrm{BER}_{\text {out }}$ curve find the corresponding decoder output $\operatorname{BER}\left(P_{\text {Dec,out }}\right)$.

(6) Find the corresponding $m_{i}$ from (5-9). ${ }^{13}$

(7) Calculate $\Delta_{i}=E^{i}\left(\bar{x}_{n}^{2}\right)$ using (5-10).

(8) Compute $\Sigma_{i+1}^{2}$ from (7-5).

(9) Go to step (3).

The primary result for using the BERT chart for a non-adaptive TFDE with MSP mapping is presented in Figure 7-5.

In the continuation of this work we intend to investigate the possibility of improving the BERT chart result and examining this method for other bit to symbol mappings. We can also consider the possibility of calculating the function $\mathfrak{I}$ theoretically.

\footnotetext{
${ }^{13}$ For clarification in this chapter we show the decoder output BER by $P_{D e c, \text { out }}$ instead of $P_{i}$. Therefore in (5-9), $P_{i}$ should be replaced by $P_{\text {Dec,out }}$.
} 


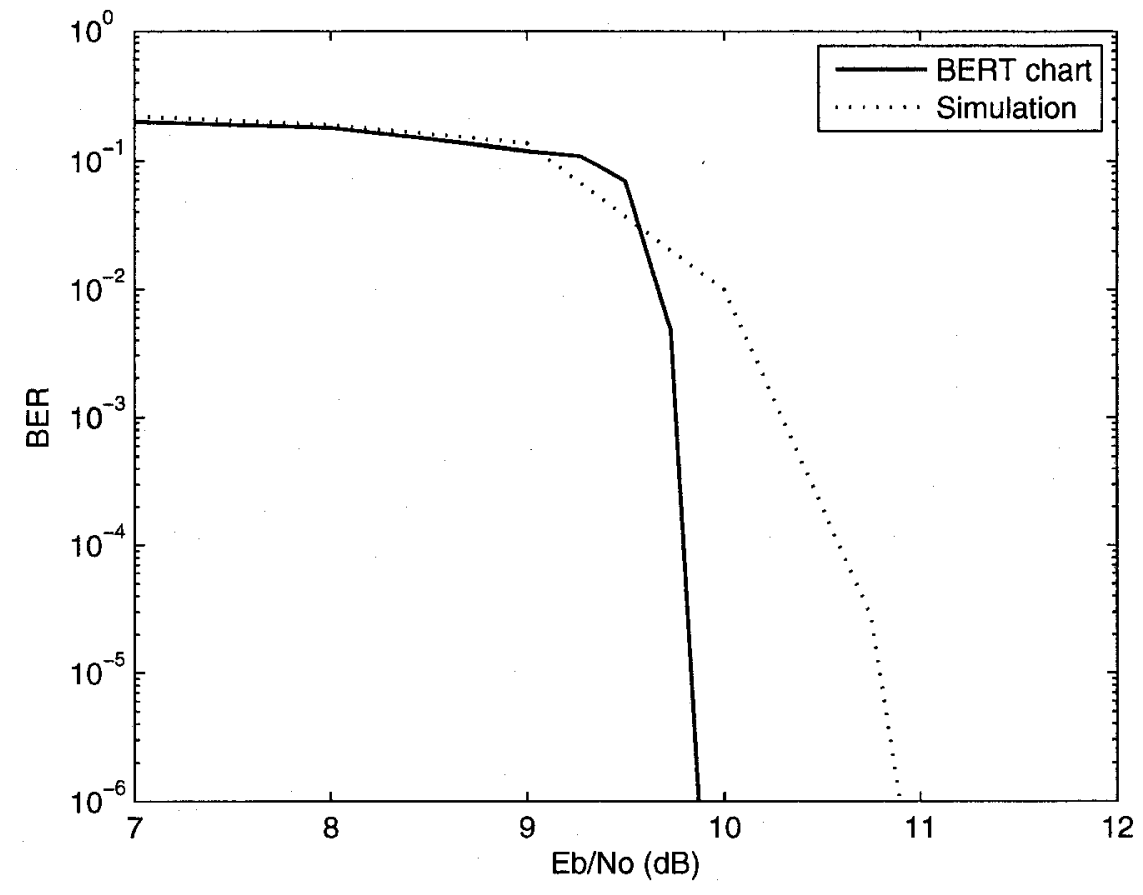

Figure 7-5. BERT chart result for ID-TFDE. 


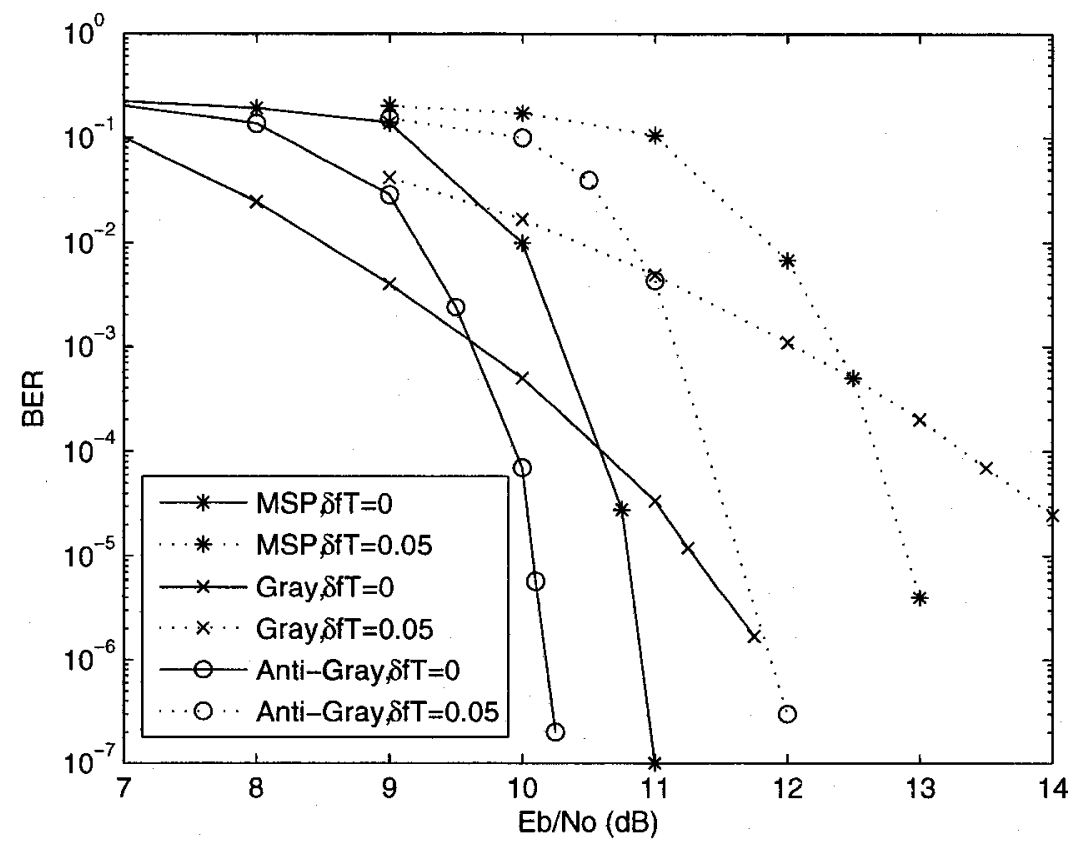

Figure 7-6. Effect of frequency offset on the performance of ID-TFDE, 16-QAM constellation.

\subsubsection{Sensitivity of ID-TFDE with different mappings to frequency offset}

Any deviation in the oscillator frequency increases the error probability or decreases the a priori information reliability. As aforementioned, the sensitivity of various mappings to reliability of the feed-back information is different such that when the reliability improves mappings like MSP and anti-Gray outperform Gray. Therefore we expect that frequency offset generates different performance degradations for ID-TFDE with different mappings. The primary result for frequency offset of $\delta f T=0.05$ is presented in Figure 7-6. The simulation parameters are the same as in Chapter 5. As can be seen, the performance degradation at BER of $10^{-4}$ is $1.5,2$ and $2.6 \mathrm{~dB}$ for anti-Gray, MSP and Gray mapping.

A possible future work in the context of synchronization is investigating the efficiency of different synchronization methods to improve the performance of ID-TFDE. 


\subsection{Conclusion}

In this chapter we reviewed the considered problems in this thesis. We briefly introduced ID-TFDE and the efficiency of using this method in iterative systems. Finally we considered the possibility of extending the research in this thesis about TFDE to IDTFDE. 


\section{Appendix A Matrix Inversion Lemma}

Matrix Inversion Lemma (MIL) [103]:

$$
(\mathbf{A}+\mathbf{B C D})^{-1}=\mathbf{A}^{-1}-\mathbf{A}^{-1} \mathbf{B}\left(\mathbf{D} \mathbf{A}^{-1} \mathbf{B}+\mathbf{C}^{-1}\right)^{-1} \mathbf{D} \mathbf{A}^{-1},
$$

where A, B, C and D are assumed to be $N \times N, N \times L, L \times L$ and $L \times N$ matrices. We also assumed that the inverses exist.

A special case of MIL is known as Woodbury's identity:

$$
\left(\mathbf{A}+\mathbf{C} \mathbf{C}^{H}\right)^{-1}=\mathbf{A}^{-1}-\frac{\mathbf{A}^{-1} \mathbf{C} \mathbf{C}^{H} \mathbf{A}^{-1}}{1+\mathbf{C}^{H} \mathbf{A}^{-1} \mathbf{C}}
$$




\section{Appendix B Non-adaptive Frequency Domain}

\section{Equalizer}

If there is no a priori information available, $\lambda_{n}=0$ and $\bar{x}_{n}=0$. Thus the diagonal elements of $\mathbf{v}_{n}$ are $v_{n}=\sigma_{d}^{2}-\left|\bar{x}_{n}\right|^{2}=\sigma_{d}^{2}$ and $\mathbf{v}_{n}=\sigma_{d}^{2} I_{N \times N}$. Therefore (4-9) changes to:

$$
\mathbf{w}_{n}^{*}=\left(\sigma^{2} \mathbf{I}_{N \times N}+\sigma_{d}^{2} \mathbf{C}_{h} \mathbf{C}_{h}^{H}\right)^{-1} \widetilde{\mathbf{s}} \sigma_{d}^{2}
$$

Since $\mathbf{C}_{h}$ is as follows

$$
\mathbf{C}_{h}=\left[\begin{array}{llllllll}
h_{0} & 0 & 0 \cdots 0 & h_{N_{h}-1} & \cdots \cdots & h_{2} & h_{1} \\
h_{1} & h_{0} & 0 & \cdots & 0 & h_{N_{h}-1} \cdots \cdots & h_{2} \\
\vdots & h_{1} & h_{0} & 0 & & & \\
h_{N_{h}-1} & \vdots & & & & & h_{3} \\
0 & h_{N_{h}-1} & \ddots & & & & \\
0 & 0 & & & & & \\
\vdots & \vdots & & & & & \\
0 & 0 & \cdots \cdots 0 & h_{N_{h}-1} \cdots & & & h_{1} & h_{0}
\end{array}\right],
$$

the result of multiplication of $\mathbf{C}_{h}$ by the FFT matrix (F) with elements of $F_{i, k}=\exp \left(-j \frac{2 \pi(i-1)(k-1)}{N}\right)$ for $k, i=1, \ldots, N$ is: 


$$
\mathbf{F C}_{h}=\left[\begin{array}{lrrrr}
H_{1} & e^{-j \frac{2 \pi 1 \times 0}{N}} H_{1} & e^{-j \frac{2 \pi 2 \times 0}{N}} H_{1} \cdots \cdots e^{-j \frac{2 \pi(N-1) \times 0}{N}} H_{1} \\
H_{2} & e^{-j \frac{2 \pi 1 \times 1}{N}} H_{2} & & & \vdots \\
\vdots & & \ddots & & \\
\vdots & & \ddots & & \\
H_{N} & e^{-j \frac{2 \pi 1 \times(N-1)}{N}} H_{N} \cdots \cdots & & e^{-j \frac{2 \pi(N-1) \times(N-1)}{N}} H_{N}
\end{array}\right],
$$

where $\mathbf{H}=\left[\begin{array}{ll}H_{1} & H_{2} \ldots H_{N}\end{array}\right]$ is the channel frequency response. The element of $m$-th row and $n$-th column $\left(\mathrm{X}_{m, n}\right)$ of multiplication $\mathbf{F C}_{h} \mathbf{F}^{-1}$ is $\mathrm{X}_{m, n}=H_{m} \delta_{m-n}$. Therefore we can write it as:

$$
\mathbf{F C}_{h} \mathbf{F}^{-1}=\operatorname{diag}(\mathbf{H})
$$

Equivalently we have

$$
\mathbf{C}_{h}=\mathbf{F}^{-1} \operatorname{diag}(\mathbf{H}) \mathbf{F}
$$

Considering $\mathbf{F}^{H}=\frac{1}{N} \mathbf{F}^{-1}$, we can rewrite $\mathbf{C}_{h} \mathbf{C}_{h}^{H}$ as:

$$
\begin{aligned}
\mathbf{C}_{h} \mathbf{C}_{h}^{H} & =\mathbf{F}^{-1} \operatorname{diag}(\mathbf{H}) \mathbf{F} \mathbf{F}^{H}(\operatorname{diag}(\mathbf{H}))^{H}\left(\mathbf{F}^{-1}\right)^{H} \\
& =\mathbf{F}^{-1} \operatorname{diag}(\mathbf{H}) \operatorname{diag}\left(\mathbf{H}^{*}\right) \mathbf{F}
\end{aligned}
$$

Using the Matrix Inversion Lemma we have:

$$
\left(\sigma^{2} \mathbf{I}_{N \times N}+\sigma_{d}^{2} \mathbf{C}_{h} \mathbf{C}_{h}^{H}\right)^{-1}=\left(\sigma^{2} \mathbf{I}_{N x N}+\sigma_{d}^{2} \mathbf{F}^{-1} \operatorname{diag}(\mathbf{H})(\operatorname{diag}(\mathbf{H}))^{H} \mathbf{F}\right)^{-1} .
$$

The frequency domain equalizer vector is:

$$
\begin{aligned}
\mathbf{F W}_{n}^{*} & =\mathbf{F}\left(\sigma^{2} \mathbf{I}_{N x N}+\sigma_{d}^{2} \mathbf{C}_{h} \mathbf{C}_{h}^{\prime \prime}\right)^{-1} \mathbf{C}_{h}\left[\begin{array}{ll}
1 & \mathbf{0}_{1 \times(N-1)}
\end{array}\right]^{T} \sigma_{d}^{2} \\
& =\mathbf{F}\left(\sigma^{2} \mathbf{I}_{N x N}+\sigma_{d}^{2} \mathbf{F}^{-1} \operatorname{diag}(\mathbf{H})(\operatorname{diag}(\mathbf{H}))^{H} \mathbf{F}\right)^{-1} \mathbf{F}^{-1} \operatorname{diag}(\mathbf{H}) \mathbf{F}\left[1 \quad \mathbf{0}_{1 \times(N-1)}\right]^{T} \sigma_{d}^{2}
\end{aligned}
$$

As we have $\mathbf{F}\left[\begin{array}{ll}1 & \mathbf{0}_{1 \times(N-1)}\end{array}\right]^{T}=\mathbf{1}_{N \times N}^{T}$, 


$$
\begin{aligned}
\mathbf{F}_{n}^{*} & =\sigma_{d}^{2}\left(\mathbf{F}\left(\sigma^{2} \mathbf{I}_{N \times N}+\sigma_{d}^{2} \mathbf{F}^{-1} \operatorname{diag}(\mathbf{H})(\operatorname{diag}(\mathbf{H}))^{H} \mathbf{F}\right) \mathbf{F}^{-1}\right)^{-1} \operatorname{diag}(\mathbf{H}) \mathbf{1}_{N \times N}^{T} \\
& =\sigma_{d}^{2}\left(\sigma^{2} \mathbf{I}_{N \times N}+\sigma_{d}^{2} \operatorname{diag}(\mathbf{H})(\operatorname{diag}(\mathbf{H}))^{H}\right)^{-1} \operatorname{diag}(\mathbf{H}) \mathbf{1}_{N \times N}^{T} \\
& =\operatorname{diag}\left(\mathbf{W}^{*}\right) \mathbf{1}_{N \times N}^{T} .
\end{aligned}
$$

The filter coefficients in the frequency domain are:

$$
W_{k}=\frac{H_{k}^{*}}{\frac{\sigma^{2}}{\sigma_{d}^{2}}+\left|H_{k}\right|^{2}}, k=1, \ldots, N .
$$




\section{Appendix C Adaptive Frequency Domain}

\section{Equalizer}

To achieve better performance than non-adaptive TFDE, we can use a priori information from the decoder. This results in a more accurate solution for MMSE filter coefficients. To be able to perform the frequency domain equalization, we replace $\mathbf{v}_{n}$ by $\mathbf{v}_{n}=v \mathbf{I}_{N \times N}$ where $v=\frac{1}{N} \sum_{n=1}^{N} v_{n} \cdot{ }^{14}$ The value of $v$ changes during iterations based on the performance of the decoder.

For BPSK and 16-QAM constellation, $v$ can be computed as presented in (C-1) and (C-2) respectively:

$$
\begin{gathered}
v=1-\frac{1}{N} \sum_{n=1}^{N}\left|\bar{x}_{n}\right|^{2} \\
v=\frac{1}{N} \sum_{n=1}^{N}\left[4 \tanh \left(\frac{\lambda_{n, 2}}{2}\right)+4 \tanh \left(\frac{\lambda_{n, 4}}{2}\right)+10\right]-\frac{1}{N} \sum_{n=1}^{N}\left|\bar{x}_{n}\right|^{2} .
\end{gathered}
$$

By replacing $\mathbf{v}_{n}=v I_{N \times N}$ in (4-1), the filter coefficient vector in the time domain would be:

\footnotetext{
${ }^{14}$ We assume that all soft estimated symbols have the same variance $(v)$ at a given iteration. This variance is equal to the average of soft estimated symbols' variances.
} 


$$
\mathbf{w}_{n}=\left(\left(\sigma^{2} \mathbf{I}_{N \times N}+v \mathbf{C}_{h} \mathbf{C}_{h}^{H}+\left(\sigma_{d}^{2}-v\right) \widetilde{\mathbf{s}} \widetilde{\mathbf{s}}^{H}\right)^{-1} \widetilde{\mathbf{s}} \sigma_{d}^{2}\right)^{*}
$$

Let us define $\stackrel{\Delta}{\mathbf{A}}=\sigma^{2} \mathbf{I}_{N \times N}+v \mathbf{C}_{h} \mathbf{C}_{h}^{H}$. If we apply the matrix inversion lemma to (C-3), we will have:

$$
\begin{aligned}
\mathbf{w}_{n}^{*} & =\left(\mathbf{A}+\left(\sigma_{d}^{2}-v\right) \widetilde{\mathbf{s}} \widetilde{\mathbf{s}}^{H}\right)^{-1} \widetilde{\mathbf{s}} \sigma_{d}^{2} \\
& =\left(\mathbf{A}^{-1}-\mathbf{A}^{-1} \widetilde{\mathbf{s}}\left(\left(\sigma_{d}^{2}-v\right)^{-1}+\widetilde{\mathbf{s}}^{H} \mathbf{A}^{-1} \widetilde{\mathbf{s}}\right)^{-1} \widetilde{\mathbf{s}}^{H} \mathbf{A}^{-1}\right) \widetilde{\mathbf{s}} \sigma_{d}^{2} \\
& =\mathbf{A}^{-1} \widetilde{\mathbf{s}} \sigma_{d}^{2}-\frac{\sigma_{d}^{2}}{\left(\sigma_{d}^{2}-v\right)^{-1}+\widetilde{\mathbf{s}}^{H} \mathbf{A}^{-1} \widetilde{\mathbf{s}}} \mathbf{A}^{-1} \widetilde{\mathbf{s}} \widetilde{\mathbf{s}}^{H} \mathbf{A}^{-1} \widetilde{\mathbf{s}} \\
& =\frac{1}{1+\left(\sigma_{d}^{2}-v\right) \widetilde{\mathbf{s}}^{H} \mathbf{A}^{-1} \widetilde{\mathbf{s}}} \mathbf{A}^{-1} \widetilde{\mathbf{s}} \sigma_{d}^{2} \\
& =\mathrm{KA}^{-1} \widetilde{\mathbf{s}},
\end{aligned}
$$

where $\mathrm{K}=\frac{\sigma_{d}^{2}}{1+\left(\sigma_{d}^{2}-v\right) \widetilde{\mathbf{s}}^{H} \mathbf{A}^{-1} \widetilde{\mathbf{s}}}$.

By multiplying the equation for $\mathbf{w}_{n}$ by $\mathbf{F}$ by a similar approach to that of non-adaptive equalization, we can find the filter coefficients as:

$$
W_{k}=\mathrm{K} \frac{H_{k}^{*}}{\sigma^{2}+v H_{k} H_{k}^{*}}=\mathrm{K} \frac{H_{k}^{*}}{\sigma^{2}+v\left|H_{k}\right|^{2}} .
$$

The estimated symbol in the frequency domain at the output of the FDE would be:

$$
Z_{k}=\mathrm{K} \frac{H_{k}^{*}}{\sigma^{2}+v\left|H_{k}\right|^{2}} R_{k}-\mathrm{K} \frac{\left|H_{k}\right|^{2}}{\sigma^{2}+v\left|H_{k}\right|^{2}} \bar{X}_{k}+\mu \bar{X}_{k} \text {, }
$$

where

$$
\mu=\frac{1}{N} \sum_{k=1}^{N} H_{k} W_{k}=\frac{\mathrm{K}}{N} \sum_{k=1}^{N} \frac{\left|H_{k}\right|^{2}}{\sigma^{2}+v\left|H_{k}\right|^{2}} .
$$


The coefficient $\mathrm{K}$ is a constant which can be omitted. If we change the criterion from minimizing mean squared error to minimizing average mean squared error, without using the approximation of $\mathbf{v}_{n}=v I_{N \times N}$, we achieve the exact same filter coefficients. 


\section{Appendix D Variance of the equalizer output}

We denote the variance of the equalizer output at $i$-th iteration by $\Sigma_{i}^{2}$. Therefore

$$
\Sigma_{i}^{2}=E\left(z_{k} z_{k}^{*}\right)-E\left(z_{k}\right)^{2}=E\left(z_{k} z_{k}^{*}\right)-|\mu|^{2} \sigma_{d}^{2} .
$$

Since $\mathbf{Z}=\left[Z_{1} \ldots Z_{N}\right]=F F T(\mathbf{z})$, we can express $E\left(z_{n} z_{l}^{*}\right)$ as:

$$
E\left(z_{n} z_{l}^{*}\right)=\frac{1}{N^{2}} \sum_{k} \sum_{m} E\left(Z_{k} Z_{m}^{*}\right) \exp \left(j \frac{2 \pi((n-1)(k-1)-(l-1)(m-1))}{N}\right) .
$$

Using (4-13), $E\left(Z_{k} Z_{m}^{*}\right)$ is:

$$
\begin{aligned}
E\left(Z_{k} Z_{m}^{*}\right) & =H_{k} W_{k} H_{m}^{*} W_{m}^{*} E\left(X_{k} X_{m}^{*}\right) \\
& +H_{m}^{*} W_{m}^{*}\left(\mu-H_{k} W_{k}\right) E\left(\bar{X}_{k} X_{m}^{*}\right) \\
& +H_{k} W_{k}\left(\mu^{*}-H_{m}^{*} W_{m}^{*}\right) E\left(X_{k} \bar{X}_{m}^{*}\right) \\
& +\left(\mu-H_{k} W_{k}\right)\left(\mu^{*}-H_{m}^{*} W_{m}^{*}\right) E\left(\bar{X}_{k} \bar{X}_{m}^{*}\right) \\
& +W_{k} W_{m}^{*} E\left(N_{k}^{\prime} N_{m}^{\prime^{*}}\right),
\end{aligned}
$$

where $\mathbf{N}^{\prime}$ is the noise vector in the frequency domain. Assuming that each soft estimated symbol is only correlated with the corresponding transmitted symbol, we will have the following equations:

$$
\begin{aligned}
& E\left(X_{k} X_{m}^{*}\right)=N \sigma_{d}^{2} \delta_{m-k}, \\
& E\left(\bar{X}_{k} X_{m}^{*}\right)=N E\left(\bar{x}_{n} x_{n}^{*}\right) \delta_{m-k}, \\
& E\left(X_{k} \bar{X}_{m}^{*}\right)=N E\left(\bar{x}_{n}^{*} x_{n}\right) \delta_{m-k}, \\
& E\left(\bar{X}_{k} \bar{X}_{m}^{*}\right)=N E\left(\bar{x}_{n} \bar{x}_{n}^{*}\right) \delta_{m-k},
\end{aligned}
$$

where $\delta$ is the Dirac delta function. Therefore $E\left(z_{n} z_{l}^{*}\right)$ can be expressed as: 


$$
\begin{aligned}
E\left(z_{n} z_{l}^{*}\right)= & \frac{\sigma_{d}^{2}}{N} \sum_{k}\left|H_{k}\right|^{2}\left|W_{k}\right|^{2} e^{j \frac{2 \pi(k-1)(n-l)}{N}} \\
& +\frac{1}{N} \sum_{k} H_{k}^{*} W_{k}^{*}\left(\mu-H_{k} W_{k}\right) E\left(\bar{x}_{m} x_{m}^{*}\right) e^{j \frac{2 \pi(k-1)(n-l)}{N}} \\
& +\frac{1}{N} \sum_{k} H_{k} W_{k}\left(\mu^{*}-H_{k}^{*} W_{k}^{*}\right) E\left(\bar{x}_{m}^{*} x_{m}\right) e^{j \frac{2 \pi(k-1)(n-l)}{N}} \\
& +\frac{1}{N} \sum_{k}\left|\mu-H_{k} W_{k}\right|^{2} E\left(\left|\bar{x}_{m}\right|^{2}\right) e^{j \frac{2 \pi(k-1)(n-l)}{N}} \\
& +\frac{1}{N} \sigma^{2} \sum_{k}\left|W_{k}\right|^{2} e^{j \frac{2 \pi(k-1)(n-l)}{N}} .
\end{aligned}
$$

If we define $\zeta=\frac{1}{N} \sum_{n=1}^{N}\left|W_{k}\right|^{2}$ and $\rho=\frac{1}{N} \sum_{n=1}^{N}\left|H_{k} W_{k}\right|^{2}, \Sigma_{i}^{2}$ would be:

$$
\begin{aligned}
\Sigma_{i}^{2} & =\sigma_{d}^{2} \rho+\sigma^{2} \zeta-\sigma_{d}^{2}|\mu|^{2} \\
& +\frac{1}{N} \sum_{k=1}^{N} H_{k}^{*} W_{k}^{*}\left(\mu-H_{k} W_{k}\right) E^{i-1}\left(\bar{x}_{m} x_{m}^{*}\right) \\
& +\frac{1}{N} \sum_{k=1}^{N} H_{k} W_{k}\left(\mu-H_{k} W_{k}\right)^{*} E^{i-1}\left(\bar{x}_{m}^{*} x_{m}\right) \\
& +\frac{1}{N} \sum_{k=1}^{N}\left|\mu-H_{k} W_{k}\right|^{2} E^{i-1}\left(\left|\bar{x}_{m}\right|^{2}\right),
\end{aligned}
$$

where $E^{i}($.$) denotes the expectation at the i$-th iteration. 


\section{Appendix E Calculating $E\left(\bar{x}_{n} \bar{x}_{n}^{*}\right)$}

Using (4-8), we can express $E\left(\bar{x}_{n} \bar{x}_{n}^{*}\right)$ for 16-QAM as:

$$
\begin{aligned}
E\left(\bar{x}_{n} \bar{x}_{n}^{*}\right) & =E\left(\tanh ^{2}\left(\frac{\lambda_{n, 3}}{2}\right)\right) E\left(\left(2+\tanh \left(\frac{\lambda_{n, 4}}{2}\right)\right)^{2}\right) \\
& +E\left(\tanh ^{2}\left(\frac{\lambda_{n, 1}}{2}\right)\right) E\left(\left(2+\tanh \left(\frac{\lambda_{n, 2}}{2}\right)\right)^{2}\right)
\end{aligned}
$$

Since all bit LLRs generated by the decoder have the same distribution, we remove the indices and denote the conditional pdf of LLRs by $f(\lambda \mid b)$ where $b \in\{0,1\}$. We know that $f(\lambda \mid 1)=f(-\lambda \mid 0)$, therefore

$$
E\left(\tanh ^{2}\left(\frac{\lambda}{2}\right) \mid 0\right)=E\left(\tanh ^{2}\left(\frac{\lambda}{2}\right) \mid 1\right)=\Delta
$$

where $\Delta$ is defined in $(5-12)$. We also note that:

$$
E\left(\tanh \left(\frac{\lambda}{2}\right) \mid 0\right)=-E\left(\tanh \left(\frac{\lambda}{2}\right) \mid 1\right)=\Delta .
$$

Therefore in (E-1), for the first and third bits we have:

$$
E\left(\tanh ^{2}\left(\frac{\lambda_{n, 1}}{2}\right)\right)=E\left(\tanh ^{2}\left(\frac{\lambda_{n, 3}}{2}\right)\right)=\Delta .
$$

The $E($.$) terms in (E-1) associated with the second and forth bits can be expressed as:$

$$
\begin{aligned}
& E\left(\left(2+\tanh \left(\frac{\lambda}{2}\right)\right)^{2} \mid 0\right)=4+4 \Delta+\Delta=4+5 \Delta, \\
& E\left(\left(2+\tanh \left(\frac{\lambda}{2}\right)\right)^{2} \mid 1\right)=4-4 \Delta+\Delta=4-3 \Delta .
\end{aligned}
$$

Therefore we have $E\left(\bar{x}_{n} \bar{x}_{n}^{*}\right)=8 \Delta+2 \Delta^{2}$. By a similar approach we can show that:

$$
E\left(x_{n} \bar{x}_{n}^{*}\right)=E\left(x_{n}^{*} \bar{x}_{n}\right)=\sum_{x_{n} \in S} E\left(x_{n} \bar{x}_{n}^{*} \mid x_{n}\right) p\left(x_{n}\right)=8 \Delta+2 \Delta^{2}
$$




\section{Appendix F BERT chart results for Channel C2}

To show the efficiency of the BERT chart method for other channel models, we show the results of this method for the WINNER channel model (C2) in Figure F-1. The parameters of the WINNER channel model (C2) are presented in Table F-1.

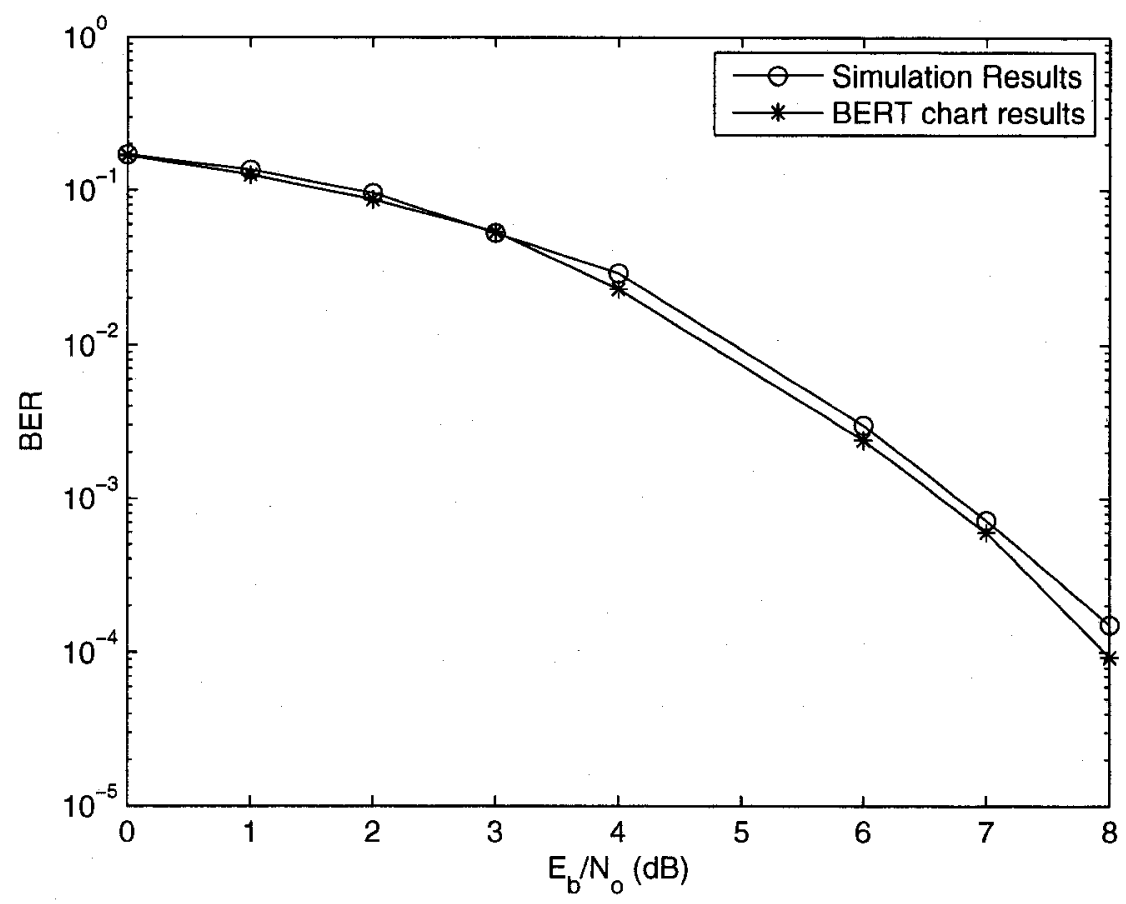

Figure F-1. BERT chart results for channel C2, BPSK constellation.

Table F-1 WINNER Channel model (C2)

\begin{tabular}{|l|l|l|l|l|l|l|l|l|l|l|}
\hline Delay $(n \mathrm{nsc})$ & 0.0 & 5 & 135 & 160 & 215 & 260 & 385 & 400 & 530 & 540 \\
\hline Power (dB) & -0.5 & 0 & -3.4 & -2.8 & -4.6 & -0.9 & -6.7 & -4.5 & -9.0 & -7.8 \\
\hline
\end{tabular}

\begin{tabular}{|l|l|l|l|l|l|l|l|l|l|l|}
\hline Delay (nsec) & 650 & 670 & 720 & 750 & 800 & 945 & 1035 & 1185 & 1390 & 1470 \\
\hline Power (dB) & -7.4 & -8.4 & -11 & -9 & -5.1 & -6.7 & -12.1 & -13.2 & -13.7 & -19.8 \\
\hline
\end{tabular}




\section{Appendix G Synchronization performance for BPSK constellation in channel C2}

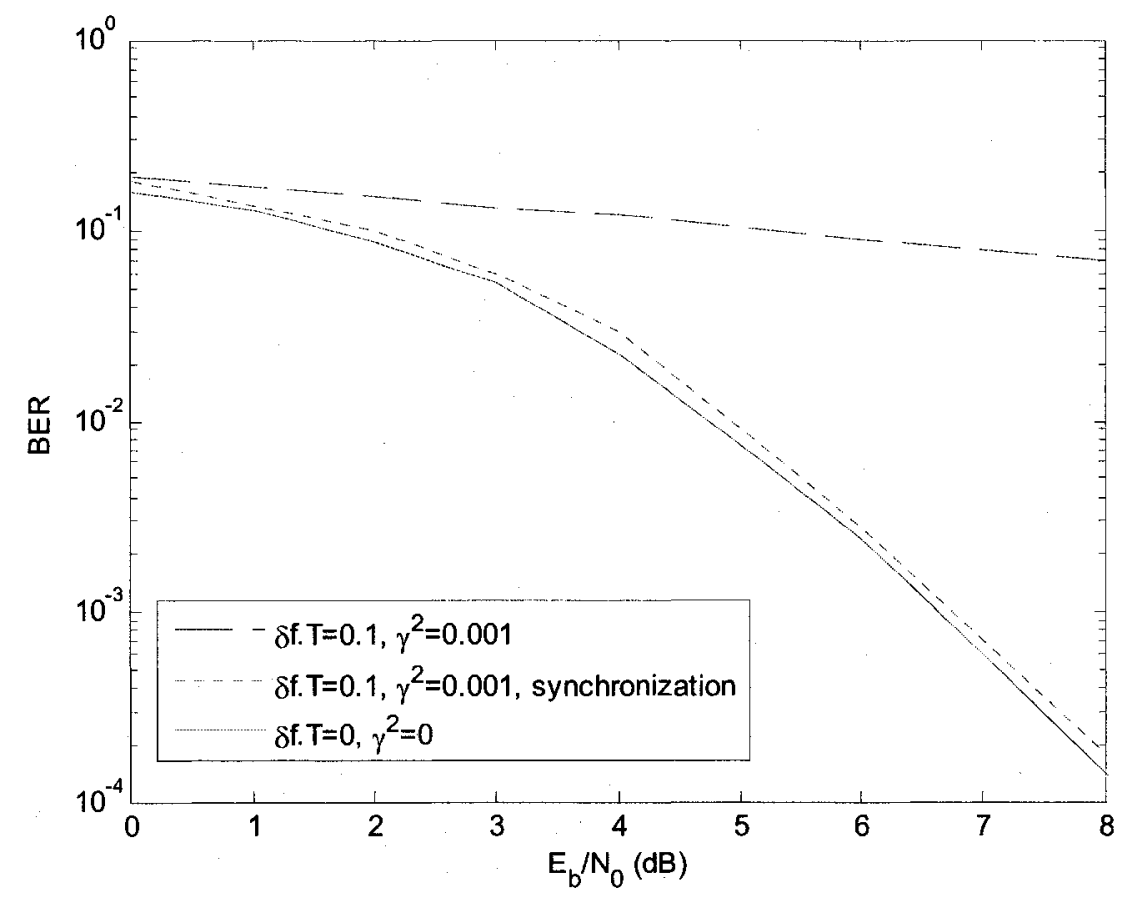

Figure G-1. The performance of synchronization method over channel C2 for BPSK. 


\section{References}

[1] J. G. Proakis, Digital Communications, McGraw-Hill, Boston, 2000.

[2] H. Sari, G. Karam, and I. Jeanclaude, "Transmission for Digital Terrestrial TV Broadcasting," IEEE Commun. Mag., vol. 33, pp. 100-109, Feb. 1995.

[3] R. Chang and R. Gibby, "A Theoretical Study of Performance of an Orthogonal Multiplexing Transmission Scheme," IEEE Trans. Commun., vol. 16, pp. 529540, Aug. 1968.

[4] S. Weinstein and P. Ebert, "Data Transmission by Frequency-Division Multiplexing Using the Discrete Fourier Transform," IEEE Trans. Commun., vol. 19, pp. 628-634, Oct. 1971.

[5] C.S. Lee, S.X. Ng, L. Piazzo, and L. Hanzo, "TCM, TTCM, BICM and iterative BICM assisted OFDM-based digital video broadcasting to mobile receivers," in Proc. IEEE Vehicular Tech. Conf.(VTC), May 2001, pp. 732-736.

[6] E. Zehavi, "8-PSK trellis codes for a Rayleigh channel," IEEE Trans. Commun., vol. 40, pp. 873-884, May 1992.

[7] Hansson and T. Aulin, "Channel symbol expansion diversity-improved coded modulation for the Rayleigh fading channel," in Proc. Int. Conf. Commun. (ICC), vol. 2, June 1996, pp.891-895.

[8] X. Li and L. J. Cimini, "Effects of clipping and filtering on the performance of OFDM," in Proc. IEEE Vehicular Tech. Conf. (VTC), May 1997, pp. 1634-1638.

[9] P. Van Eetvelt, G. Wade, and M. Tomlinson, "Peak to average power reduction for OFDM schemes by selective scrambling," Electronic letters, pp 1963-1964, Oct. 1996. 
[10] K.G. Paterson and V. Tarokh, "On the existence and construction of good codes with low peak-to-average power ratios," IEEE International Symposium on Inform. Theory (ISIT), 25-30 June 2000, pp. 217.

[11] Brajal and A. Chouly, "Compensation of nonlinear distortions for orthogonal multicarrier schemes using predistortion," in Proc. Global Telecommun. Conf., Nov. 28-Dec. 2, 1994, pp. 1909-1914.

[12] H. Sari, G. Karam, and I. Jeanclaud, "Frequency-domain equalization of mobile radio and terrestrial broadcast channels," in Proc. Global Telecommun. Conf., Nov. 28-Dec. 2, 1994, pp. 1-5.

[13] D. Falconer, S.L. Ariyavisitakul, A. Benyamin-Seeyar, and B. Eidson, "Frequency domain equalization for single-carrier broadband wireless systems," IEEE Commun. Mag., vol. 40, pp. 58 - 66, Apr. 2002.

[14] D. Falconer and S.L. Ariyavisitakul, "Broadband wireless using single carrier and frequency domain equalization," in Proc. $5^{\text {th }}$ International Symposium on Wireless Personal Multimedia Commun., Oct. 2002, pp. 27-36.

[15] Z. Wang, X. Ma and G.B. Giannakis, "OFDM or single-carrier block transmissions?," IEEE Trans. Commun., vol. 52, no. 3, pp. 380-394, Mar. 2004.

[16] M. Sabbaghian and D. Falconer, "Reducing Required Power Back-Off of Nonlinear Amplifiers in Serial Modulation using SLM Method," in Proc. Vehicular Tech. Conf., Dallas, TX, Sept. 2005.

[17] M. Sabbaghian and D. Falconer, "Peak to Average Power Ratio Properties of MC-CDMA and SM-CDMA," in Proc. Vehicular Tech. Conf., Melbourne, Australia, May 2006. 
[18] M. Sabbaghian and D. Falconer, "Peak to Average Power Ratio properties of SMCDMA," to be submitted to IEEE Trans. Vehicular Tech.

[19] F. Danilo-Lemoine, D. Falconer, C-T Lam , M. Sabbaghian, and K. Wesołowski, "Power Backoff Reduction Techniques for Generalized Multicarrier Waveforms," submitted to EURASIP journal on Wireless Communications and Networking, Apr. 2007.

[20] D. Falconer, F. Danilo-Lemoine, C. Lam, and M. Sabbaghian, "Power Backoff Reduction for Generalized Multicarrier Waveforms," in Proc. EUSIPCO 2007.

[21] M. Sabbaghian and D. Falconer, "Comparison between Convolutional and LDPC Code based Turbo Frequency Domain Equalization," in Proc. International Conf. Commun., Istanbul, Turkey, June 2006.

[22] M. Sabbaghian, D. Falconer and H. Saeedi, "BER Transfer Chart Analysis of Turbo Frequency Domain Equalization," in Proc. Vehicular Tech. Conf., Montreal, Canada, Sept. 2006.

[23] M. Sabbaghian and D. Falconer, "Comparison of adaptive and non-adaptive turbo frequency domain equalization via BER transfer chart," in Proc. Vehicular Tech. Conf., Baltimore, MD, USA, Sept. 2007.

[24] M. Sabbbaghian and D. Falconer, "BER Transfer Chart Analysis of Turbo Frequency Domain Equalization," submitted to IEEE Trans. Wireless Commun, Jan. 2007.

[25] M. Sabbaghian and D. Falconer, "Joint Turbo Frequency Domain Equalization and Blind Carrier Synchronization," in Proc. CWIT, Jun. 2007. 
[26] M. Sabbaghian and D. Falconer, "Joint Turbo frequency Domain Equalization and Carrier Synchronization," accepted in IEEE Trans. Wireless Commun., Jun. 2007.

[27] R. Van Nee and R. Prasad, OFDM for Wireless Multimedia Communications, Norwood. Artech House, 2000.

[28] P. Struhsaker and K. Griffin, “Analysis of PHY Waveform Peak to Mean Ratio and Impact on RF Amplification," contribution to IEEE 802.16.3c-01/46, Mar. 2001.

[29] M. Pauli and P. Kuchenbecker, "On the reduction of the out-of-band radiation of OFDM-signals," in Proc. International Conf. Commun. (ICC), vol.1, June 1998, pp. 1304-1308.

[30] H. Ochiai and H. Imai, "On the distribution of the peak-to-average power ratio in OFDM signals," IEEE Trans. Commun., vol. 49, pp.282-289, Feb. 2001.

[31] H. Ochiai and H. Imai, "Performance analysis of deliberately clipped OFDM signals," IEEE Trans. Commun., vol. 50, pp. 89-101, Jan. 2002.

[32] S. H. Muller and J. B. Huber, "A comparison of peak power reduction schemes for OFDM," in Proc. Global Telecommun. Conf., Nov. 1997, pp. 1-5.

[33] S. H. Muller and J. B. Huber, "A novel peak power reduction scheme for OFDM," IEEE Int. Symp. On Personal Indoor and Mobile Radio Commun., Sept. 1997, pp.1090-1094.

[34] T. A. Wilkinson and A. E. Jones, "Minimization of the peak to mean envelope power ratio of multicarrier transmission schemes by block coding," IEEE Vehicular Tech. Conf. (VTC), July 1995, pp. 825-829. 
[35] T. Pollet, M. Van Bladel, and M. Moeneclaey, "BER sensitivity of OFDM systems to carrier frequency offset and Wiener phase noise," IEEE. Trans. Commun., vol. 43, pp.191-193, Feb.-Apr. 1995.

[36] T. Pollet and M. Moeneclaey, "Synchronizability of OFDM signals," Global Telecommun. Conf., vol.3 Singapore, Nov. 1995, pp. 2054-2058.

[37] L. Tomba, "On the effect of Wiener phase noise in OFDM systems," IEEE Trans. Commun., vol. 46, pp. 580-583, May1998.

[38] P. H. Moose, "A technique for orthogonal frequency division multiplexing frequency offset correction," IEEE Trans. Commun., vol. 42, pp. 2908-2914, Oct. 1994.

[39] J. J. van de Beek, M. Sandell, M. Isaksson, and P. O. Boriesson, "Low-complex frame synchronization in OFDM systems," $4^{\text {th }}$ IEEE Int. Conf. on Universal Personal Commun., Nov. 1995, pp. 982-986.

[40] J. J. van de Beek, M. Sandell and P. O. Boriesson, "ML estimation of time and frequency offset in OFDM systems," IEEE. Tran. Signal Processing, vol. 45, pp. 1800-1805, July 1997.

[41] J. Shentu, K. Panta, and J. Armstrong, "Effects of phase noise on performance of OFDM systems using an ICI cancellation scheme," IEEE Trans. Broadcasting, vol. 49, pp. 221-224, June 2003.

[42] L. Piazzo and P. Mandarini, "Analysis of phase noise effects in OFDM modems," IEEE Trans. Commun., vol. 50, pp. 1696-1705, Oct. 2002. 
[43] K. Wesolowski and J. Pochmara, "Efficient algorithm for adjustment of adaptive predistorter in OFDM transmitter," in Proc. IEEE Vehicular Tech. Conference (VTC), Sept. 2000, pp. 2491-2496.

[44] Ma Xiaoqiang, H. Kobayashi, and S.C. Schwartz, "Joint frequency offset and channel estimation for OFDM," in Proc. Global Telecommun. Conf., Dec. 2003, pp. $15-19$.

[45] C. Douillard, C.B. Michel Jezequel, C. Berrou and A. Picart, P. Didier and A. Glavieux, "Iterative correction of intersymbol interference: Turbo equalization," European Trans. Telecommun., vol. 6, pp. 507-511, Sept. 1995.

[46] M. Tuchler, A. Singer, and R. Koetter, "Minimum mean squared error equalization using a priori information”, IEEE Trans. Signal Processing, vol. 50, pp. 673-683, Mar. 2002.

[47] M. Tuchler and J. Hagenauer, "Turbo Equalization using frequency domain equalizers," in Proc. Allerton Conf., Oct. 2000.

[48] M. Tuchler and J. Hagenauer, "Linear time and frequency domain turbo equalization," in Proc. IEEE Vehicular Tech. Conf., May 2001, pp. 1449-1453.

[49] B. Ng, C. Lam, and D. Falconer, "Turbo Frequency Domain Equalization for Single-carrier Broadband Wireless System," in IEEE Trans. Wireless, vol. 6, No. 2, Feb., 2007, pp. 759-767.

[50] K. R. Narayanan, Wang Xiaodong, and Yue Guosen, "Estimating the PDF of the SIC-MMSE equalizer output and its applications in designing LDPC codes with turbo equalization," IEEE Trans. Wireless Commun., vol. 4, pp. 278-287, Jan. 2005. 
[51] A. Dholakia, E. Eleftheriou, T. Mittelholzer, and M. P. C. Fossorier, "Capacityapproaching codes: can they be applied to the magnetic recording channel?," IEEE Commun. Mag., vol. 42, pp. 122-130, Feb. 2004.

[52] J. H. Gunther, M. Ankapura, and T.K. Moon, “A generalized LDPC decoder for blind turbo equalization," IEEE Tran. Signal processing, vol. 53, partl, pp. 38473856, Oct. 2005.

[53] S. Ten Brink, "Convergence behavior of iteratively decoded parallel concatenated codes," IEEE Trans. Commun., vol. 49, pp. 1727-1737, October 2001.

[54] S. Ten Brink, G. Kramer, and A. Ashikhmin, "Design of low-density parity check codes for modulation and detection," IEEE Trans. Commun., vol. 52, no. 4, pp. 670-678, Apr. 2004.

[55] M. Tuchler, R. Koetter, and A. Singer, "Turbo equalization: Principles and new results," IEEE Trans. Commun., vol. 50, pp. 754-767, May 2002.

[56] Seok-Jun Lee and A. C. Singer, "Linear Turbo Equalization Analysis via BER Transfer and Exit Charts," IEEE Trans. Signal Processing, vol. 53, pp.2883-2897, Aug. 2005.

[57] T. Arauio and R. Dinis, "Iterative equalization and carrier synchronization for single-carrier transmission over severe time-dispersive channels," in Proc. Global Telecommun. Conf., vol.5, Dec. 2004, pp. 3103-3107.

[58] A. R. Nayak, J. R. Barry and S. W. McLaughlin, "Joint timing recovery and turbo equalization for partial response channels," Magnetic Conf., May 2002. 
[59] C. Rapp, "Effects of HPA-Nonlinearity on a 4-DPSK/OFDM-Signal for a Digital Sound Broadcasting System," in Proc. the Second European Conf. Satellite Commun., Liege, Belgium, Oct. 22-24, 1991, pp. 179-184.

[60] M. Honkanen and S. G. Haggman, "New aspects on nonlinear power amplifier modeling in radio communication system simulations," in Proc. PIMRC Conf., vol.3, Sept. 1997 , pp. 844-848.

[61] A. A. M. Saleh, "Frequency-independent and frequency-dependent nonlinear models of TWT amplifiers," IEEE Trans. Commun., vol. 29, pp.1715-1720, Nov. 1981.

[62] A. Ghorbani and M. Sheikhan, "The effect of solid state power amplifiers (SSPAs) nonlinearities on MPSK and M-QAM signal transmission," in Proc. Int. Conf. Digital Processing of Signal in Commun., Sept. 1991, pp.193-197.

[63] WINNER Document D2.5, https://www.ist-winner.org/

[64] R. D. J. Van Nee, "OFDM codes for peak-to-average power reduction and error correction", in Proc. Global Telecommun. Conf., vol. 1, Nov. 1996, pp. 18-22.

[65] A. Mobasher and A.K. Khandani, "Integer-based constellation-shaping method for PAPR reduction in OFDM systems," IEEE Trans. Commun., vol. 54, no. 1, pp.119-127, Jan. 2006.

[66] S. Hara and R. Prasad, "Overview of multicarrier CDMA," IEEE Commun. Mag., vol. 35 , no. 12, pp. 126-133, Dec. 1997.

[67] L. Liu and F. Adachi, "Joint frequency-domain differential detection and equalization for DS-CDMA signal transmissions in a frequency-selective fading channel," in Proc. IEEE JSAC, vol. 24, pp. 649-658, Mar. 2006. 
[68] P. Xiaoming, C. Francois, T.T. Tjhung and A.S. Madhukumar, “A simplified transceiver structure for cyclic extended CDMA system with frequency domain equalization," in Proc. IEEE Vehicular Tech. Conf. , vol. 3, pp. 1753-1757, May 2005.

[69] S. Tsumura, S. Hara and Y. Hara, "Performance comparison of MC-CDMA and cyclically prefixed DS-CDMA in an uplink channel [mobile radio]," in Proc. IEEE Vehicular Tech. Conf., vol. 1, pp. 414-418, Sep. 2004.

[70] Y. Kai, A.S. Madhukumar and F. Chin, "Multistage interference cancellation with frequency domain equalization for uplink transmission of single carrier cyclic prefix assisted CDMA system," in Proc. IEEE Wireless Commun. Networking Conf., vol. 1, pp. 585-590, Mar. 2003.

[71] K. Takeda and F. Adachi, "Inter-chip interference cancellation for DS-CDMA with frequency-domain equalization," in Proc. IEEE Vehicular Tech. Conf., vol. 4, pp. 2316-2320, Sep. 2004.

[72] J. Wang and J Chen, "Performance of Wideband CDMA Systems with Complex Spreading and Imperfect Channel Estimation," in IEEE JSAC in Commun., vol. 19, No. 1, Jan. 2001.

[73] J. Oppermann and B.S. Vucetic, "Complex spreading sequences with a wide range of correlation properties," IEEE Trans. Commun., vol. 45, pp.365-375, Mar. 1997.

[74] N. Ohkubo and T. Ohtsuki, "Design criteria for phase sequences in selected mapping", in Proc. IEEE Vehicular Tech. Conf., Apr. 2003, pp. 373-377. 
[75] N. Ohkubo and T. Ohtsuki, "A peak to average power ratio reduction of multicarrier CDMA using selected mapping", in Proc. IEEE Vehicular Tech. Conf., Sept. 2002, pp. 2086-2090.

[76] J. Lin, Iterative Detection/Equalization with Frequency Domain Filtering for MIMO Channels, M.Sc. Thesis, Carleton University, Ottawa, ON., 2004.

[77] T. J. Richardson, A. Shokrollahi, and R. L. Urbanke, "Design of capacity approaching irregular low density parity check codes," IEEE Trans. Inform. Theory, vol. 47, pp. 619-637, Feb. 2001.

[78] J. R. Barry, "Low Density Parity Check Codes," A tutorial, Downloadable at: http://users.ece.gatech.edu/ barry/6606/handouts/ldpc.pdf

[79] J. Hagenauer and P. Hoeher, "A Viterbi algorithm with soft-decision outputs and its applications," in Proc. IEEE Global Telecommun. Conf., Nov. 1989, vol. 3, pp $1680-1686$.

[80] L. Bahl, J. Cocke, F. Jelinek, and J. Raviv, "Optimal decoding of linear codes for minimizing symbol error rate," IEEE Trans. Inform. Theory, vol. 20, pp 284-287, Mar. 1974.

[81] P. Robertson, E. Villebrun and P. Hoeher, "A comparison of optimal and suboptimal MAP decoding algorithms operating in the log domain," in Proc. Int. Conf. Commun. (ICC), Jun. 1995, vol. 2, pp. 1009-1013.

[82] P. Robertson, P. Hoeher, and E. Villebrun, "Optimal and sub-optimal maximum a posteriori algorithms suitable for turbo decoding," European Trans. Telecommun., vol. 8,no. 2, Mar. 1997.

[83] http://www.inference.phy.cam.ac.uk/mackay/codes/data.html 
[84] S. D. Morgera, "Efficient synthesis and implementation of large discrete Fourier transforms," SIAM Journal Computing, vol. 9, No. 2, pp. 251-272, 1980.

[85] S. C. Chan and K. L. Ho, "On indexing the prime-factor fast Fourier transform algorithm," IEEE Trans. Circuits and Systems, vol. 38, No. 8, pp.951-953, Aug. 1991.

[86] S. Winograd, "On computing the discrete Fourier transform," Math. Computation, vol. 32 , pp.175-199, 1978.

[87] D. P.Kobla and T. W. Parks, "A Prime factor FFT algorithm using high speed convolution," IEEE Trans. Acoust., Speech, Signal Processing, vol. ASASP-25, pp. 281-294, Aug. 1977.

[88] D. Divsalar and F. Pollara, "Multiple turbo codes for deep space communications," JPL, Pasadena, CA, TDA Progress Rep. 42-121, May 1995.

[89] K. R. Narayanan, Wang Xiaodong and Yue Guosen, "LDPC code design for turbo equalization," in Proc. IEEE Inform. Theory workshop, pp.57-60, Oct. 2002.

[90] H. El Gamal and A. R. Hammons, "Analyzing the turbo decoder using the Gaussian approximation," IEEE Trans. Inform. Theory, vol. 47, no. 2, pp. 671686, Feb. 2001.

[91] F. Lehmann and G. M. Maggio, "Analysis of the iterative decoding of LDPC and product codes using the Gaussian approximation," IEEE Trans. Inform. Theory, vol. 49, no. 11, pp. 2993-3000, Nov. 2003.

[92] M. Tuchler, S. ten Brink, and J. Hagenauer, "Measures for tracing convergence of iterative decoding algorithms," in Proc. 4th Int. ITG Conf. Source and Channel Coding, Berlin, Germany, Jan. 2002, pp. 53-60. 
[93] R. Dinis, A. Gusmão, and N. Esteves, "Iterative block-DFE techniques for singlecarrier-based broadband communications with transmit/receive space diversity," ISWCS 2004, Sept. 2004, pp. 347-351.

[94] R. Dinis, A. Gusmão, and N. Esteves, "On broadband block transmission over strongly frequency-selective fading channels," in Proc. Wireless 2003, Calgary, Canada, July 2003.

[95] T. J. Richardson and R. L. Urbanke, "The capacity of low-density parity-check codes under message-passing decoding," IEEE Trans. Inform. Theory, vol. 47, pp. 599-618, Feb. 2001.

[96] E. Sharon, A. Ashikhmin, and S. Litsyn, "EXIT functions for the Gaussian channel," in Proc. 40th Annu. Allerton Conf. Communication, Control, Computers Allerton, IL, Oct. 2003, pp. 972-981.

[97] H. Meyr and G. Ascheid, Synchronization in digital communications, Wiley, New York, 1990.

[98] T. M. Schmidl and D. C. Cox, "Robust frequency and timing synchronization for OFDM," IEEE. Trans. Commun., vol. 45, pp.1613-1621, Dec. 1997.

[99] A. G. Armada and M. Calvo, "Phase noise and sub-carrier spacing effects on the performance of an OFDM communication system," IEEE Commun. Letter, vol. 2, pp.11-13, Jan. 1998.

[100] D. Falconer and J. Salz, "Optimal reception of digital data over the Gaussian channel with unknown delay and phase jitter," IEEE Trans. Inform. Theory, vol. 23, pp. 117-126, Jan. 1977. 
[101] M. Luise, M. Marselli and R. reggianini, "Low-Complexity Blind Carrier Frequency Recovery for OFDM Signals over Frequency-Selective Radio Channels," IEEE Trans. Commun., vol. 50, no. 7, pp. 1182-1188, July 2002.

[102] M. Oerder and H. Meyr, "Digital filter and square timing recovery," IEEE Trans. Commun., vol. 36, pp. 605-612, May 1988.

[103] S. Haykin, Adaptive Filter Theory, Prentice-Hall, Englewood Cliffs, N.J., $2^{\text {nd }}$ edition, 1991. 